\title{
TU/e EmonOWEN

\section{On the stability of rotor-and-bearing systems and on the calculation of sliding bearings}

\section{Citation for published version (APA):}

Reinhoudt, J. P. (1972). On the stability of rotor-and-bearing systems and on the calculation of sliding bearings. [Phd Thesis 1 (Research TU/e / Graduation TU/e), Mechanical Engineering]. Technische Hogeschool Eindhoven. https://doi.org/10.6100/IR109125

DOI:

10.6100/IR109125

Document status and date:

Published: 01/01/1972

\section{Document Version:}

Publisher's PDF, also known as Version of Record (includes final page, issue and volume numbers)

\section{Please check the document version of this publication:}

- A submitted manuscript is the version of the article upon submission and before peer-review. There can be important differences between the submitted version and the official published version of record. People interested in the research are advised to contact the author for the final version of the publication, or visit the $\mathrm{DOI}$ to the publisher's website.

- The final author version and the galley proof are versions of the publication after peer review.

- The final published version features the final layout of the paper including the volume, issue and page numbers.

Link to publication

\section{General rights}

Copyright and moral rights for the publications made accessible in the public portal are retained by the authors and/or other copyright owners and it is a condition of accessing publications that users recognise and abide by the legal requirements associated with these rights.

- Users may download and print one copy of any publication from the public portal for the purpose of private study or research.

- You may not further distribute the material or use it for any profit-making activity or commercial gain

- You may freely distribute the URL identifying the publication in the public portal.

If the publication is distributed under the terms of Article 25fa of the Dutch Copyright Act, indicated by the "Taverne" license above, please follow below link for the End User Agreement:

www.tue.nl/taverne

Take down policy

If you believe that this document breaches copyright please contact us at:

openaccess@tue.nl

providing details and we will investigate your claim. 


\section{ON THE STABILITY OF ROTOR-AND-BEARING SYSTEMS AND ON THE CALCULATION OF SLIDING BEARINGS}




\section{ON THE STABILITY OF ROTOR-AND-BEARING SYSTEMS AND ON THE CALCULATION OF SLIDING BEARINGS}

\section{PROEFSCHRIFT}

TER VERKRIJGING VAN DE GRAAD VAN DOCTOR IN DE TECHNISCHE WETENSCHAPPEN AAN DE TECHNISCHE HOGESCHOOL EINDHOVEN, TEN OVERSTAAN VAN EEN COMMISSIE AANGEWEZEN DOOR HET COLLEGE VAN DECANEN, OP VRIJDAG 25 FEBRUARI 1972 TE 16 UUR IN HET OPENBAAR TE VERDEDIGEN

DOOR

JACOBUS PIETER REINHOUDT

GEBOREN TE VLISSINGEN 
DIT PROEFSCHRIFT IS GOEDGEKEURD DOOR DE PROMOTOR PROF. IR. W. L. ESMEIJER EN DE COPROMOTOR PROF. IR. H. BLOK 


\section{Acknowledgement}

The work described in this thesis was performed at the Philips Research Laboratories, Eindhoven, in the research group headed by Prof. Dr. Ir. E. A. Muijderman. I feel greatly indebted to the management of the laboratories, and in particular to Prof. Dr. Ir. J. A. Haringx, for affording me the opportunity of publishing this work as a thesis.

My sincere thanks are due to all those who have contributed in one way or another to the completion of these studies, and especially to Mr. J. G. G. Bos for his manifold help, to Ir. J. Bootsma for the stimulating discussions in relation to the finite-element method, and to Mr. H. G. E. Wallace for his critical remarks and painstaking revision of the text. 


\section{CONTENTS}

1. GENERAL INTRODUCTION . . . . . . . . . . . . 1

1.1. High-speed rotors; bearings . . . . . . . . . . . . . . . . 1

1.2. Stability of rotor-and-bearing systems . . . . . . . . . 2

1.3. Methods for determining the stability . . . . . . . . . . . . 3

1.4. State of the art . . . . . . . . . . . . . 4

1.5. The goal of the present investigations . . . . . . . . 6

References ................. . . 7

2. THE STABILITY OF A SYMMETRIC ROTOR-AND-BEARING SYSTEM IN WHICH BOTH THE ROTOR AND BEARING SUPPORTS ARE RIGID . . . . . . . . . . . . . . . . . 8

2.1. Introduction . . . . . . . . . . . . . . . . 8

2.2. Stability of a symmetric rotor-and-bearing system with statically loaded bearings ... . . . . . . . . . . . 9 9

2.2.1. Equations of motion and characteristic equation . . . . . 9

2.2.2. Application to a rotor with smooth journal bearings . . . 15

2.3. Stability of a symmetric rotor-and-bearing system with a constant bearing load rotating synchronously with the shaft . . . . 17

2.3.1. Equations of motion . . . . . . . . . . . . 17

2.3.2. Application to a rotor with smooth journal bearings . . . 19

References ... . . . . . . . . . . . . . 21

3. LINEAR AND NONLINEAR ASPECTS OF THE STABILITY OF A SYMMETRIC ROTOR-AND-BEARING SYSTEM WITH RIGID BEARING SUPPORTS AND ROTATIONALLY SYMMETRIC BEARING RESPONSE . . . . . . . . . . . . . . . 22

3.1. Introduction . . . . . . . . . . . . . . . . . . . 22

3.2. Types of bearings with rotationally symmetric response . . . . 22

3.3. A stability criterion of the case of rotationally symmetric response 24

3.4. Physical interpretation . . . . . . . . . . . . 26

3.4.1. Physical meaning of the parameters . . . . . . . . 26

3.4.2. Equations of motion in polar coordinates . . . . . . . 27

3.5. The effect of large bearing displacements . . . . . . . . 28

3.5.1. The possibility of a circular orbit . . . . . . . . . . 28

3.5.2. The stability of the circular orbit ... . . . . . . 30

3.6. Conclusions and supplementary remarks . . . . . . . . . . . 31 
4. THE EFFECT OF FLEXIBLE BEARING SUPPORTS ON THE STABILITY OF A SYMMETRIC ROTOR-AND-BEARING SYSTEM

4.1. Introduction . . . . . . . . . . . . . . . . . . . 33

4.2. The expected behaviour of a flexible bearing support consisting of springs and dampers . . . . . . . . . . . . . . . . . 34

4.3. Supports consisting of springs and dampers . . . . . . . . . 37

4.3.1. Equations of motion . . . . . . . . . . . . . 37

4.3.2. The choice of the dimensionless support parameters . . . 40

4.3.3. The characteristic equation if the mass of the support and gyroscopic effects are negligible . . . . . . . . . . . 40

4.3.4. Application to an ALG bearing and supports having rotationally symmetric response . . . . . . . . . . 42

4.3.5. Application to an ORS bearing and supports having rotationally symmetric response . . . . . . . . . . . . . 43

4.3.6. Example of the application of the stability diagrams . . 49

4.3.7. Possibilities of designing a flexible support . . . . . . 50

4.3.8. Asymmetric supports . . . . . . . . . . . . . . . . 51

4.4. Simplified method of calculation applicable if the bearings as well as the supports have rotational symmetry . . . . . . . . . 53

4.4.1. Analysis . . . . . . . . . . . . . . . . . . 53

4.4.2. Examples . . . . . . . . . . . . . . . . 56

4.5. Bearing supported by an additional bearing (floating-bush bearing) .

4.5.1. Equations of motion and characteristic equation . . . . 57

4.5.2. Approximate torque balance for the determination of the angular velocity of the bush ......... 60

4.5.3. Application to ORS bearings . . . . . . . . . . . 61

4.5.4. Floating-bush bearing with a freely chosen bush speed . . 62

4.5.5. Floating-bush bearing with smooth bearings . . . . . 63

References . . . . . . . . . . . . . . . 64

5. THE FINITE-ELEMENT METHOD FOR THE CALCULATION OF SLIDING BEARINGS . . . . . . . . . . . . . . 65

5.1. Introduction . . . . . . . . . . . . . . . . 65

5.2. Principles of the method . . . . . . . . . . . . . 66

5.3. The finite-element method based on the "ordinary" Reynolds equation. . . . . . . . . . . . . . . 68

5.3.1. The Reynolds differential equation . . . . . . . . . 68

5.3.2. Transformation of the Reynolds equation into a variation integral . . . . . . . . . . . . 70

5.3.3. Boundary conditions . . . . . . . . . . 71 
5.3.4. Introduction of the finite elements . . . . . . . 73

5.3.5. Application of the boundary conditions . . . . . . . . 77

5.4. The finite-element method based on the "generalised" Reynolds equation. . . . . . . . . . . . . 78

5.4.1. The generalised Reynolds equation . . . . . . . . 78

5.4.2. Transformation of the generalised Reynolds equation into a variation integral . . . . . . . . . 85

5.4.3. The integrals $I_{-3}, I_{-2}, I_{+1}$ and $I_{+3}$ for rectangular and triangular grooves . . . . . . . . . . . . . 87

5.5. Example of an element: the triangular element . . . . . . . . 88

References ................... . . 90

6. CAVITATION ............................ 91

6.1. Introduction . . . . . . . . . . . . . . 91

6.2. Cavitation conditions . . . . . . . . . . . . . . . 92

6.2.1. Conditions for smooth journal bearings . . . . . . 92

6.2.2. Modification of the Jakobson and Floberg conditions for grooved bearings . . . . . . . . . . . . 94

6.3. The dummy-flow method . . . . . . . . . . . . . . . . . 96

6.4. Examples . . . . . . . . . . . . . . . . . . . . 98

6.4.1. Effect of the parameter $p_{\text {cav }}$ on the load capacity of a smooth journal bearing with circumferential feeding . . . 98

6.4.2. Effect of the parameter $p_{\text {cav }}$ on a helical-groove bearing . 102

6.5. Dynamically loaded bearings . . . . . . . . . . . . 103

References . . . . . . . . . . . . . . 103

7. ACCURACY OF THE FINITE-ELEMENT METHOD AND RESULTS OF CALCULATIONS OF LOAD CAPACITY, RESPONSE COEFFICIENTS AND STABILITY OF VARIOUS TYPES OF BEARINGS . . . . . . . . . . . . . . . . . . . 104

7.1. Introduction . . . . . . . . . . . . . 104

7.2. Short description of the computer program . . . . . . . 105

7.3. Inaccuracies of the finite-element method . . . . . . . . 106

7.3.1. Sources of errors and principles for determining the inaccuracy . . . . . . . . . . . . . . 106

7.3.2. Comparison with the Sommerfeld and the Reynolds solution . . . . . . . . . . . . . . . . . 107

7.3.3. Comparison with the results of Sassenfeld and Walther . 108

7.3.4. The accuracy of the pressure build-up in a grooved, centric, journal bearing ................... . . .

7.3.5. Comparison with analytical results of a spherical spiralgroove thrust bearing. 
7.4. Load capacity, response coefficients and stability diagrams of some types of bearings

7.4.1. Determination of the response coefficients

7.4.2. Smooth journal bearing with axial lubricant-supply groove (ALG bearing)

7.4.3. Smooth journal bearing with circumferential oil supply (CLG bearing)

7.4.4. Grooved journal bearing with optimum radial stiffness (ORS bearing)

7.4.5. The spherical spiral-groove bearing

7.4.6. Spherical spiral-groove bearing with optimum axial thrust (SOAT bearing)

7.4.7. Spherical spiral-groove bearing with optimum radial stiffness (SORS bearing)

8. DISCUSSION, CONCLUSIONS AND RECOMMENDATIONS OF FURTHER INVESTIGATIONS . . . . . . . . . . . . . 128

8.1. Discussion . . . . . . . . . . . . . . . . . . . . . . . . 128

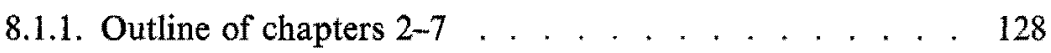

8.1.2. Conclusions . . . . . . . . . . . . . . . . 132

8.2. Final remarks . . . . . . . . . . . . . . . . . . . 132

8.2.1. Flexible rotors . . . . . . . . . . . . . . . . 132

8.2.2. Asymmetric rotor . . . . . . . . . . . . . . 133

8.2.3. Experimental verification. . . . . . . . . . . . 134

8.2.4. Computer program for transforming the characteristic determinant into the characteristic equation . . . . . 134

8.3. Possibilities of further investigations and development . . . . 135

8.3.1. Extension of the above theories . . . . . . . . . . 135

8.3.2. New forms of sliding bearings and of rotor-and-bearing systems . . . . . . . . . . . . . . . . 135

References . . . . . . . . . . . . . . 136

Appendix I $\ldots \ldots \ldots \ldots$

Appendix II . . . . . . . . . . . . . . . . . . . . . . . . . 140

Appendix III . . . . . . . . . . . . . . . . . . . 141

List of Symbols . . . . . . . . . . . . . . . . . 147

Summary . . . . . . . . . . . . . . . 153 


\section{GENERAL INTRODUCTION}

\subsection{High-speed rotors; bearings}

High-speed rotors have been finding ever wider application in the last few decades. There has been a steady expansion of their field of application, and attempts are being made to raise the speed of existing types to ever increasing values. Examples of fast-running rotors are to be found, for instance, in navigational systems, in some types of electric motors, in centrifuges and ultracentrifuges, in turbines and compressors. High-speed rotors are used also in production machines, and sometimes a new production technique cannot be implemented until a rotor-and-bearing system capable of reaching the required speed is available. Examples of this aspect are to be found in the textile industry, in the manufacture of filaments for incandescent lamps, and in the enrichment of uranium in the ultracentrifuge.

Depending on the kind of application, there are various reasons for raising the speed. For example, a certain angular momentum is usually required of rotors in a navigational system: the mass, overall dimensions, or both, can be reduced if the speed of operation is increased. With centrifuges the attainable centripetal acceleration, and hence the separating effect, increases rapidly with speed. With rotary-flow machines, e.g. the so-called expanders, ever smaller units are demanded: as the circumferential speed must remain approximately constant, this again leads to an increase in angular velocity.

If a type of bearing has to be selected for a rotor-and-bearing system, then the required life of the bearings often plays a major role. The service life of the common rolling-element bearings, such as ball bearings, is normally limited by fatigue and wear; the attainable life decreases in proportion as load and speed increase. A sliding bearing, however, is an element on which the load and speed have relatively little effect as far as the service life is concerned. This is due to the characteristic feature of this type of bearing that the bearing surfaces are, under normal operation conditions, fully separated by a film of gas or liquid. Thus mechanical wear is prevented and the life of a sliding bearing, even at high speed and under heavy load, can be very long. For instance, in applications where the specifications of speed or load, or both, are severe, a sliding bearing may be a good alternative to a rolling-element bearing or even offer the only appropriate solution.

Sliding bearings can be divided into hydrostatic and hydrodynamic (selfacting) bearings, or into gas-lubricated and liquid-lubricated bearings.

The self-acting, liquid-lubricated bearing (for example, the oil-lubricated bearing) is in many cases better suited for application in high-speed rotor-andbearing systems than the other types, and while the theories developed in this report often have a wide applicability, special attention will be paid to such 
self-acting, liquid-lubricated bearings. Let us examine this statement a little more closely.

In hydrostatic bearings the lubricant has to be supplied under pressure from an external pressure source, while in self-acting bearings the pressure, from which the bearing derives its load capacity, is generated in the bearing itself. Thus a system with self-acting bearings may be less complicated.

Gas has a low viscosity as compared with liquids. As a result a self-acting gas bearing must have a larger size, for the same load capacity, than a selfacting liquid bearing (cf. "volume rule", Vogelpohl $\left.{ }^{1-18}\right)$ ) and as the size has a greater effect than the viscosity on the energy consumption of the fluid bearing, this consumption tends to be higher in the case of a gas bearing. Moreover, the boundary-lubrication properties, which become important when a bearing is overloaded, heavily favour oil in comparison with, for example, gas.

A major advantage of gas lubrication is that a gas-lubricated system can often work in the lubricating medium itself, thus simplifying the problems of sealing and lubricant supply of the bearings. This advantage, however, has lately been compensated in part by the development of simple and effective seals for oil(or grease-)lubricated self-acting bearings ${ }^{1-22}$ ). This makes it possible to provide such a bearing with a "lubricant supply for life".

Considering the vast literature on gas-lubricated bearings, the liquid-lubricated bearing seems to have been neglected in the last twenty years as a bearing for high-speed rotors. The author is of the opinion, however, that the latter bearing opens up great possibilities for high-speed rotors and deserves more attention than it has received hitherto.

\subsection{Stability of rotor-and-bearing systems}

In the foregoing the point was made that, at heavy loads and high speeds, a sliding bearing is often superior to a rolling-element bearing as far as the service life of the system is concerned. Yet in most applications it is not possible simply to replace a rolling-element bearing by a sliding bearing, even were the construction to allow of this. For one thing, a sliding bearing affects the dynamics of the rotor in a far more complex way than does a rolling-element bearing: sliding bearings can readily induce self-excited vibrations in a rotor-andbearing system and these vibrations may give rise to damage of the bearings and the rotor. In the case of liquid-lubricated bearings the phenomenon is often referred to as "oil whirl", "half-frequency whirl", or "resonant whirl" (see e.g. Newkirk $\left.{ }^{1-19}\right)$ ). The onset of self-excited vibrations is caused by the fact that the rotor-and-bearing system becomes unstable. Because the instabilities occur more readily as the dynamic effects become stronger, for instance at higher speeds, one may say that the application of sliding bearings in high-speed rotors literally stands or falls with the possibilities of controlling the dynamics of the system in such a way as to avoid instabilities. 
Let us examine this in more detail and consider a rotor loaded by a static force (cf. fig. 2.1). This rotor may take up a position in its bearings such that the forces generated in the bearings are just in equilibrium with the load. But this equilibrium position may be unstable, by which we mean that, if we disturb the equilibrium situation by giving the rotor and initial deflection with respect to its equilibrium position, the rotor does not return to the equilibrium position but will move further and further away from it.

In a stability analysis the motion about the equilibrium position is investigated, to determine whether the rotor, after an initial disturbance, returns to the equilibrium position (stable) or not (unstable).

In chapter 2 the following set of equations, describing the motion of a certain rotor about the equilibrium position will be derived (cf. eq. (2.13)):

$$
\begin{aligned}
& \frac{1}{\bar{W}} \ddot{x}+b_{x x} \dot{x}+a_{x x} x+\left(b_{x y}+\frac{\lambda}{\bar{W}}\right) \dot{y}+a_{x y} y=0, \\
& \frac{1}{\bar{W}} \ddot{y}+b_{x y} \dot{y}+a_{y y} y+\left(b_{y x}-\frac{\lambda}{\bar{W}}\right) \dot{x}+a_{y x} x=0 .
\end{aligned}
$$

Here the dimensionless coordinates $x, y$ determine the deflection of the rotor with respect to the equilibrium position. The properties of the bearings are expressed by the response coefficients $a_{x x}, a_{x y}, b_{x x}$, etc. On the assumption that the deflections are small, the bearing response may be linearised and then the response coefficients are constants. $1 / \bar{W}$ may be considered as a dimensionless mass, and $\lambda$ is a measure of the gyroscopic effects. The first and second (dimensionless) time derivatives of $x$ are denoted by respectively $\dot{x}(=\mathrm{d} x / \mathrm{d} t)$ and $\ddot{x}\left(=\mathrm{d}^{2} x / \mathrm{d} t^{2}\right)$.

The goal of the stability analysis is to determine from such equations as given by (1.1) whether the rotor, after being subjected to an initial disturbance $(x=\hat{x}, y=\hat{y})$, will return to the equilibrium position $(x=0, y=0)$ or not.

\subsection{Methods for determining the stability}

If the equations of motion are linear, as in (1.1), we may distinguish three methods for determining the stability.

Method 1. Starting from an initial disturbance $(\hat{x}, \hat{y})$ and with given values of $\bar{W}$ and $\lambda$, we calculate the motion by integration of $(1.1)$ with respect to time $(\tau)$ and then check whether this motion converges to $(0,0)$ or not.

Method 2. As the equations (1.1) are linear we may use the substitution

$$
x=\hat{x} \exp (s \tau), \quad y=\hat{y} \exp (s \tau),
$$

and deduce the characteristic determinant and characteristic equation of 
(1.1) (cf. eq. (2.15)). The quantities $s$ of (1.2) are the roots of the characteristic equation. By calculating all the roots we are able to check whether all the roots have negative real parts (stable) or not.

Method 3. For the determination of the stability it is not necessary to calculate all the roots, but on applying the Routh-Hurwitz stability criterium (see e.g. Malkin ${ }^{1-7}$ )) to the characteristic equation we can check whether the roots lie in the left half of the complex plane (stable) or not.

Method 1 (cf. e.g. Elrod $\left.{ }^{1-23}\right)$ ) provides much extra information about the motion and is not restricted to linear equations of motion. However, especially at the border of stability, the solving of the problem by this method may require a great deal of time because of the difficulties of establishing convergence or divergence.

Method 2 gives also much additional information about the motion and may be equally tedious. A variant of this method, the zero-point method, utilises the fact that, at the boundary of stability, a pair of purely imaginary roots, $s$, should exist and thus the boundary curves of stability may be calculated. As the existence of such a pair of roots is a necessary but insufficient condition, it is always necessary to check whether the remaining roots lie in the left half of the complex plane (cf. appendix I).

Method 3 gives less information but works very quickly if only the characteristic equation is known (methods 1 and 2 can be used without deducing this equation). In this work method 3 will mainly be used.

\subsection{State of the art}

It is almost impossible and not very meaningful, within the scope of this work, to give a historical review of the literature published in the field of rotorand-bearing dynamics. For this the reader is referred to the existing reviews ${ }^{1-2 / 6}$ ). Here we shall be concerned particularly with the stability aspect of the rotor-and-bearing dynamics; a survey of this subordinate field will be found in Sternlicht et al. ${ }^{1-1}$ ). Recent progress in development can be outlined as follows.

(A) The feasibility of calculating the performance of a sliding bearing is rapidly increasing, particularly because of the advent of large, fast computers, so that the effect of a certain bearing on the stability of a rotor can be determined more accurately than before.

(B) Designs well known for their favourable influence on rotor stability, such as tilting-pad bearings, flexible supports, etc., have been and are still being investigated, both theoretically and experimentally.

Concerning the greater feasibility of calculation, the Reynolds equation for 
an incompressible flow in the lubricant film has in many recent publications been solved by means of a finite-difference method. It proved possible to modify the Reynolds equation in such a way that turbulence in the lubricant film could be taken into account ${ }^{1-20,21}$ ). In 1967, Orcutt and Arwas ${ }^{1-8}$ ) gave the coefficients of bearing response of smooth, fully circular and partial-arc bearings with laminar and turbulent films.

In articles by Hirs ${ }^{1-10}$ ) (1964) and Vohr and Chow ${ }^{1-11}$ ) (1965) journal bearings with helical grooves were calculated for small values of the eccentricity and it was pointed out that bearings of this kind were stabler than smooth journal bearings. In 1969 Chow and Vohr ${ }^{1-9}$ ) published the results of calculations of helical-groove journal bearings working with a laminar or turbulent film in respect of both small and large values of the eccentricity.

For quite some time use is being made in mechanics of what is known as the finite-element method, for instance for calculations of strength. Reddi ${ }^{1-12}$ ) seems to have been the first to apply this method to an incompressible lubricant film. It has, in comparison with the finite-difference method, the great advantage of being highly versatile, so that bearings with greatly differing geometries can be calculated by means of the same program of calculation.

As far as favourable constructions are concerned various experimental investigations and practical experience have shown that the bearing support plays a significant role in rotor-and-bearing dynamics and that the stability of a rotor-and-bearing system can be improved by a design providing a measure of elasticity and damping. Kerr ${ }^{1-13}$ ) gave values of the spring constant and damping obtainable by means of an O-ring support. Powell and Tempest ${ }^{1-24}$ ) reported experimental results gained with bearings supported by O-rings. Orcutt and $\mathrm{Ng}^{1-14}$ ), in their paper on floating-bush bearings, pointed out that a bearing of this kind had satisfactory stability properties, but they were unable to explain this.

Elasticity of the shaft and bearing support without damping of the flexible element in general worsens the stability. This can be seen from articles by Sternlicht, Poritsky, and Arwas ${ }^{1-25}$ ), Marsh ${ }^{1-15}$ ), and Lund ${ }^{1-16}$ ). Lund considered also the effect of damping in the support of a rotor in gas bearings. These authors all used the zero-point method but did not check the position of the not purely imaginary roots. Then there is always a risk that there are complex roots with a positive real part at the moment when a pair of roots is purely imaginary; it might then be erroneously inferred that one was dealing with a boundary between stability and instability.

Gunther ${ }^{1-17}$ ) examined the effect of the support on the stability of rotors with internal damping. Here the effect of the bearings on the dynamic behaviour of the rotor was left out of account. The results are of qualitative importance for rotors in journal bearings, as the internal damping of the rotor is found to cause the same kind of effect as does a self-acting bearing. 


\subsection{The goal of the present investigations}

Next to providing more insight into the influence of some effects, such as gyroscopic effects, the purpose of the investigations was to look for solutions that could improve the stability of a rotor-and-bearing system equipped with sliding bearings.

The stability can in principle be improved in two ways.

- One can try to remedy the real cause of instability, namely the way in which a sliding bearing reacts to deflections. This leads to looking for bearings with good stability properties or to trying to affect the bearing geometry in such a way as to yield good stability.

- One can also try to compensate, somewhere in the system, the destabilising effects of the sliding bearings, for example by using special bearing supports.

In chapter 2 a symmetrical rotor-and-bearing system with rigid rotor and rigid bearing supports is investigated. Special attention is paid to gyroscopic effects.

In chapter 3 the system of chapter 2 is again considered, but now for the case that the bearing response possesses rotational symmetry. Such symmetry simplifies the analysis and leads to a simple and easily usable stability criterion; moreover, an extrapolation to large bearing deflections and nonlinear behaviour becomes possible.

In chapter 4 the effect of flexible bearing supports on the stability of the system is investigated. The floating-bush bearing is considered as a special case of a flexible support.

In chapter 5 a versatile method for the calculation of liquid-lubricated bearings, the finite-element method, is developed. This method permits of the calculation of bearings with greatly differing geometries without any significant change in the computer procedures; it forms a tool that can be used in designing bearings with good stability properties, because it creates the possibility of rapid assessment of the effects of geometric modifications.

In chapter 6 consideration is given to the problem of how to include cavitation (which is very important in liquid bearings) in the finite-element method and, furthermore, examples are given in which the cavitation conditions frequently used in the literature lead to incorrect conclusions.

In chapter 7 the accuracy of the finite-element method is checked and several types of bearings are calculated.

Chapter 8 contains an evaluation of the work, supplementary remarks, and recommendations for further investigations.

Note. Although the theories and results are often of general applicability, so that they are valid also for gas bearings, etc., the assumption has been made throughout this work that the lubricant can be considered as an incompressible Newtonian fluid. 


\section{REFERENCES}

1-1) B. Sternlicht and N. F. Rieger, Rotor stability, Conference Lub. and Wear, London (Sept. 1967), 182, part 3A, paper no. 7.

1-2) D. D. Fuller, A review of the state-of-the-art for the design of self-acting gas-lubricated bearings, Trans. ASME, J. Lub. Techn. 91, 1-16, 1969.

1-3) T. A. Harris, Lubrication review: A digest of the literature for 1965, Trans ASME, J. Lub. Techn. 89, 1-37, 1967.

1-4) T. A. Harris, Lubrication review: A digest of the Literature for 1966, Trans. ASME, J. Lub. Techn. 90, 1-34, 1968.

1-5) J. H. Rumbarger, Lubrication review: A digest of the literature for 1967, Trans. ASME, J. Lub. Techn. 91, 225-259, 1969.

1-6) J. H. Rumbarger, Lubrication review: A digest of the literature for 1968, Trans. ASME, J. Lub. Techn. 92, 185-215, 1970.

1-7) J. G. Malkin, Theorie der Stabilitat einer Bewegung, R. Oldenburg, München, 1959.

1-8) F. K. Orcutt and E. B. Arwas, The steady-state and dynamic characteristics of a full circular bearing and a partial arc bearing in the laminar and turbulent flow regimes, Trans. ASME, J. Lub. Techn. 89, 143-153, 1967.

${ }^{1-9}$ ) C. Y. Chow and J. H. Vohr, Helical-grooved journal bearing operated in turbulent regime, ASME-ASLE Lubrication Conference, Houston, Texas (Oct. 1969), paper 69-Lub-28.

1-10) G. G. Hirs, The load capacity and stability characteristics of hydrodynamic journal bearings, Trans. ASLE 8, 296-305, 1965.

1-11) J. H. Vohr and C. Y. Chow, Characteristics of herringbone-grooved gas lubricated journal bearings, Trans. ASME, J. basic Eng. 87, 568 576, 1965.

1-12) M. M. Reddi, Finite element solution of the incompressible lubrication problem, Trans. ASME, J. Lub. Techn. 91, 524-533, 1969.

1-13) J. Kerr, The onset and cessation of half-speed whirl in air-lubricated self pressurised journal bearings, Proc. Inst. mech. Engrs 180, part 3k, 145-153, 1965/66.

1-14) F. K. Orcutt and C. W. Ng, Steady-state and dynamic properties of the floating-ring journal bearing, Trans. ASME, J. Lub. Techn. 90, 243-252, 1968.

1-15) $\mathrm{H}$. Marsh, The stability of self-acting gas journal bearings with noncircular members and additional elements of flexibility, Lubrication Symposium, Las Vegas (1968), paper 68-LubS-45.

1-16) J. W. Lund, The stability of an elastic rotor in journal bearings with flexible, damped supports, Trans. ASME, J. appl. Mech. 32, 911-920, 1965.

1-17) E. J. Gunter, Jr. and P. R. Trumpler, The influence of internal friction on the stability of high speed rotors with anisotropic supports, J. Eng. for Industry 91, $1105-1113,1969$.

1-18) G. Vogelpohl, Betriebssichere Gleitlager, Springer Verlag, Berlin, 1958.

$\left.{ }^{1-19}\right)$ B. L. Newkirk, Journal bearing instability, Proc of the Conference on Lub. and Wear, London (October 1957).

${ }^{1-20}$ ) C. W. Ng and C. H. T. Pan, A linearized turbulent lubrication theory, Trans. ASME, J. basic Eng. 87, 675-682, 1965.

1-21) G. G. Hirs, Fundamentals of a bulk-flow theory for turbulent lubricant films, Thesis Techn. Univ. Delft, 1970.

1-22) E. A. Muijderman, Self-contained grease lubricated journal bearings (to be published).

1-23) H. G. Elrod and G. A. Glanfield, Computer procedures for the design of flexibly mounted, externally pressurised, gas lubricated journal bearings, Gas Bearing Symposium Univ. Southampton (1971), paper 22.

1-24) J. W. Powell and M. C. Tempest, A study of high-speed machines with rubber stabilized air bearings, Trans. ASME, J. Lub. Techn. 90, 701-708, 1968.

1-25) B. Sternlich t, H. Poritsky and E. G. Arwas, Dynamic stability aspects of cylindrical journal bearings using compressible and incompressible fluids, Proc. First Int. Symp. on Gas-Lubricated Bearings, Washington D.C. (1959). 


\section{THE STABILITY OF A SYMMETRIC ROTOR-AND-BEARING SYSTEM IN WHICH BOTH THE ROTOR AND BEARING SUPPORTS ARE RIGID}

\subsection{Introduction}

A rotor-and-bearing system will here be considered "symmetrical" if it has a plane of symmetry (plane aa' in fig. 2.1) and if the rotor itself is rigid and rotationally symmetrical with respect to the line $\mathrm{bb}^{\prime}$. It should be understood that the external load is part of the system and should be symmetrical with respect to $\mathrm{aa}^{\prime}$, too.

The motion of this system can be resolved into two modes, viz. the "translational" mode, in which the axis $\mathrm{bb}^{\prime}$ undergoes merely a translation, and the "conical" mode, in which the centre of gravity, G, remains stationary.

For the case of a static external load, stability diagrams of the translational mode of some types of bearings are known from the literature ${ }^{2-1,2}$ ). Stability diagrams of the conical motion are also known, but here the influence of gyroscopic effects has generally been ignored $2-2,3$ ). Since in some rotor-and-bearing systems gyroscopic effects play a significant part ${ }^{2-5}$ ), they have in the present examination of the stability of a rotor been taken into account.

In this chapter the stability of the rotor with two types of external loads is investigated. In sec. 2.2 it is assumed that the external load is static. Then, in the equilibrium position, both bearings have equal static deflections, $\mathbf{E}$. Data on the stability are found by analysis of the motion about the equilibrium position. In sec. 2.3 it is assumed that the rotor is loaded by a force of constant magnitude, rotating in synchronism with the rotor (for example an unbalance

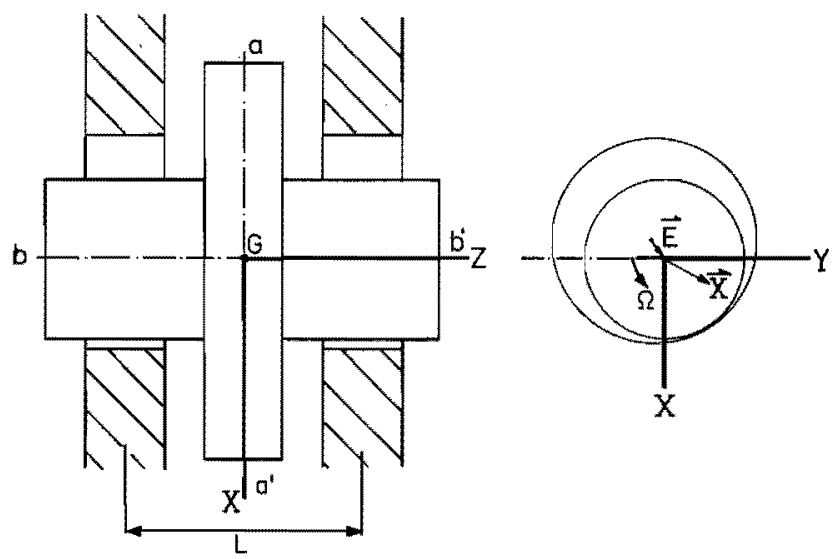

Fig. 2.1. Symmetric rotor-and-bearing system. 
force). It will further be assumed that the bearings behave rotationally symmetrically with respect to this force, so that in the equilibrium "position" both journals describe circles ( $\mathbf{E}$ rotates). Again the stability data are found by analysis of the motion about this equilibrium position.

\subsection{The stability of a symmetric rotor-and-bearing system with statically loaded bearings}

\subsubsection{Equations of motion and the characteristic equation}

Let us consider a symmetric rotor-and-bearing system (fig. 2.1) in which the rotor is loaded by a (symmetrical) static force. Let $M_{R}$ denote the mass of the rotor, $L$ the distance between the bearing centres, $I$ the moment of inertia with respect to the centre line $\left(\mathrm{bb}^{\prime}\right)$ of the rotor, and $J$ the moment of inertia with respect to a line in plane aa' passing through the centre of gravity, $G$.

In the equilibrium position of the rotor (drawn in fig. 2.1) the static deflections, $\mathbf{E}$, and the forces of reaction, F, of the two bearings will be equal. For an analysis of the stability the motion of the rotor about its equilibrium position must be investigated. If we now define a stationary system $X, Y, Z$, then the motion of the rotor may be described by the deflections of both journals with respect to this system; for the translational mode these deflections are equal, for the conical mode they have equal magnitudes but opposite directions.

If $\mathbf{X}$ is the dynamical bearing deflection and $\Delta \mathbf{F}$ the additional force of reaction due to this deflection, then for the translational mode we may write (with $X, Y, Z$ an inertial system)

$$
\Delta \mathbf{F}=\frac{M_{R}}{2} \frac{\mathrm{d}^{2} \mathbf{X}}{\mathrm{d} t^{2}}
$$

or, if we introduce the components of $\Delta \mathbf{F}$ in the $X, Y, Z$ system,

$$
\begin{aligned}
& \Delta F_{x}=\frac{M_{R}}{2} \frac{\mathrm{d}^{2} X}{\mathrm{~d} t^{2}}, \\
& \Delta F_{y}=\frac{M_{R}}{2} \frac{\mathrm{d}^{2} Y}{\mathrm{~d} t^{2}} .
\end{aligned}
$$

For the conical motion it is useful to define the system $U, V, W$ (cf. fig. 2.2): the coordinate axis $W$ coincides with the centre line of the rotor in the deflected position, $U$ and $V$ are defined by the fact that the system $X, Y, Z$ passes into the system $U, V, W$ by a rotation, through an angle $\theta$, about a line perpendicular to $Z$ and $W$. It may be assumed that in $U, V, W$ the rotor rotates solely about the $W$-axis with an angular velocity $\Omega$. 


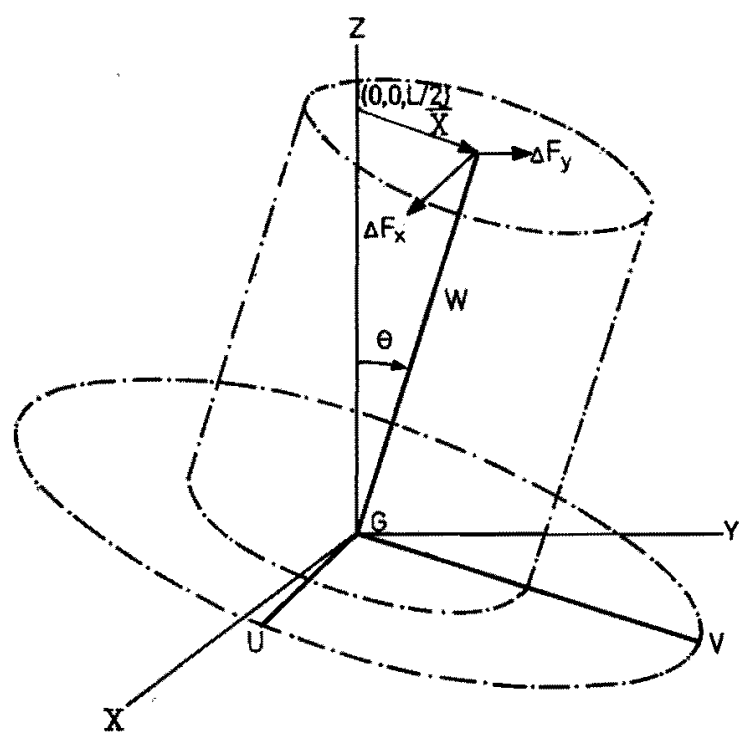

Fig. 2.2. Coordinates of the conical motion.

With $\mathbf{T}$, the tor que exerted on the rotor, $\mathbf{H}$, the angular momentum, $\boldsymbol{\Omega}_{r}$, the angular velocity of the $U, V, W$ system with respect to the $X, Y, Z$ system, and $\mathbf{e}_{j}$, the base vectors of the $U, V, W$ system, the equations of conical motion in the inertial system are

$$
\mathbf{T}=\frac{\mathrm{d} \mathbf{H}}{\mathrm{d} t}=\frac{\mathrm{d}}{\mathrm{d} t}\left(H^{j} \mathbf{e}_{j}\right),
$$

or

With

$$
\mathbf{T}=\frac{\mathrm{d} H^{j}}{\mathrm{~d} t} \mathbf{e}_{j}+H^{j} \frac{\mathrm{de}_{j}}{\mathrm{~d} t} .
$$

eq. (2.3) becomes

$$
\frac{\mathrm{de}_{j}}{\mathrm{~d} t}=\boldsymbol{\Omega}_{r} \times \mathbf{e}_{j}
$$

$$
\mathbf{T}=\frac{\mathrm{d} H^{j}}{\mathrm{~d} t} \mathbf{e}_{j}+\mathbf{\Omega}_{r} \times \mathbf{H}
$$

So far all vectors have been defined in the inertial system or, better, in the inertial "space". In a "space" in which the system $U, V, W$ is stationary, the equation of conical motion takes the following form:

$$
\mathbf{T}=\frac{\mathrm{d} \mathbf{H}}{\mathrm{d} t}+\boldsymbol{\Omega}_{r} \times \mathbf{H},
$$


and the vectors can be defined with respect to the $U, V, W$ system.

With $\theta \ll 1$, and $\mathbf{X}$ representing the deflection of one bearing, the other one being $-\mathbf{X}$, the components of $\mathbf{T}, \Omega_{r}$, and $\mathbf{H}$ in the $U, V, W$ system are

$$
\begin{aligned}
& T_{u} \approx-\Delta F_{y} L, \\
& T_{v} \approx+\Delta F_{x} L, \\
& T_{w} \approx 0 ; \\
& \Omega_{r u} \approx-\frac{2}{L} \frac{\mathrm{d} Y}{\mathrm{~d} t}, \\
& \Omega_{r v} \approx+\frac{2}{L} \frac{\mathrm{d} X}{\mathrm{~d} t}, \\
& \Omega_{r w} \approx 0 ; \\
& H_{w}=J \Omega_{r u}, \\
& H_{v}=J \Omega_{r v}, \\
& H_{w}=I\left(\Omega_{r w}+\Omega\right) .
\end{aligned}
$$

From (2.6), (2.7), (2.8) and (2.9) it follows that

$$
\begin{aligned}
& \Delta F_{x}=\frac{2 J}{L^{2}} \frac{\mathrm{d}^{2} X}{\mathrm{~d} t^{2}}+\frac{2 \Omega}{L^{2}} I \frac{\mathrm{d} Y}{\mathrm{~d} t}, \\
& \Delta F_{y}=\frac{2 J}{L^{2}} \frac{\mathrm{d}^{2} Y}{\mathrm{~d} t^{2}}-\frac{2 \Omega}{L^{2}} I \frac{\mathrm{d} X}{\mathrm{~d} t} .
\end{aligned}
$$

It is possible to combine (2.2) and (2.10) into one set of equations in which the parameters have different meanings with respect to the two modes of motion. Meanwhile introducing dimensionless magnitudes, we may write, instead of (2.2) and (2.10),

$$
\begin{aligned}
& \Delta f_{x}=\frac{1}{W}(\ddot{x}+\lambda \dot{y}), \\
& \Delta f_{y}=\frac{1}{W}(\ddot{y}-\lambda \dot{x}),
\end{aligned}
$$

in which

$$
\begin{aligned}
\Delta f_{x}=\frac{\Delta F_{x}}{F_{0}}, & \Delta f_{y}=\frac{\Delta F_{y}}{F_{0}} & \text { dimensionless forces, } \\
x=\frac{X}{\Delta R}, & y=\frac{Y}{\Delta R} & \text { dimensionless deflections, } \\
\dot{x}=\frac{\mathrm{d} x}{\mathrm{~d} \tau}, & \ddot{x}=\frac{\mathrm{d}^{2} x}{\mathrm{~d} \tau^{2}}, &
\end{aligned}
$$




$$
\begin{aligned}
& \bar{W}=\left\{\begin{array}{cl}
\frac{2 F_{0}}{M_{R} A R \Omega^{2}} & \begin{array}{l}
\text { for the translational } \\
\text { mode }
\end{array} \\
\frac{F_{0} L^{2}}{2 J \Delta R \Omega^{2}} & \text { for the conical mode }
\end{array}\right) \begin{array}{l}
\text { stability parameter } \\
\text { or reciprocal value } \\
\text { of the dimension- } \\
\text { less mass, }
\end{array} \\
& \lambda=\left\{\begin{array}{cl}
0 & \begin{array}{l}
\text { for the translational } \\
\text { mode } \\
I
\end{array}
\end{array}\right\} \begin{array}{l}
\text { gyroscopic } \\
\text { for the conical mode }
\end{array}
\end{aligned}
$$

with $\Delta R=$ radial play of bearing,

$\tau=\Omega t=$ dimensionless time,

$F_{0}=$ reference force.

A relation, the Reynolds differential equation, which describes the pressure distribution in the lubricant film is deduced in chapter 5 (eq. (5.12)). From that relation it follows that the pressure, $p$, in the lubricant depends solely on the dimensionless film thickness, $h$, and its time derivative, $\partial h / \partial \tau$ (and of course on the boundary conditions of the differential equation). In a given bearing, for example in a journal bearing, $h$ and $\partial h / \partial \tau$ depend on the position and the velocity of the journal. If the boundary conditions are fixed, or if they are a function only of the position and velocity of the journal (for example the cavitation region under quasi-static conditions, cf. chapter 6) then we may state that, for a given lubricant viscosity and angular speed, the force of reaction, $\mathbf{F}$, is a function only of the position and velocity of the journal. If we further assume that the displacements from the equilibrium position are so small that linearisation of the additional reactional forces $\Delta \mathrm{F}$ is permissible, and that we may neglect effects of misalignment (for example, due to the conical motion) we may write

$$
\begin{aligned}
& \Delta F_{x}=-A_{x x} X-B_{x x} \frac{\mathrm{d} X}{\mathrm{~d} t}-A_{x y} Y-B_{x y} \frac{\mathrm{d} Y}{\mathrm{~d} t}, \\
& \Delta F_{y}=-A_{y y} Y-B_{y y} \frac{\mathrm{d} Y}{\mathrm{~d} t}-A_{y x} X-B_{y x} \frac{\mathrm{d} X}{\mathrm{~d} t} .
\end{aligned}
$$

The response coefficients $A_{x x}, A_{x y}, B_{x x}, B_{x y}$, etc., are only functions of the equilibrium position of the journal bearing under consideration. Introducing dimensionless magnitudes we obtain

$$
\begin{aligned}
& \Delta f_{x}=-a_{x x} x-b_{x x} \dot{x}-a_{x y} y-b_{x y} \dot{y}, \\
& \Delta f_{y}=-a_{y y} y-b_{y y} \dot{y}-a_{y x} x-b_{y x} \dot{x},
\end{aligned}
$$


with

$$
\begin{aligned}
& a_{x x}=\frac{\Delta R}{F_{0}} A_{x x}, \quad a_{x y}=\frac{\Delta R}{F_{0}} A_{x y}, \quad \text { etc. } \\
& b_{x x}=\frac{\Omega \Delta R}{F_{0}} B_{x x}, b_{x y}=\frac{\Omega \Delta R}{F_{0}} B_{x y}, \text { etc. }
\end{aligned}
$$

From (2.11) and (2.12a) it follows that the two modes of motion can be described by the differential equations

$$
\begin{aligned}
& \frac{1}{\bar{W}} \ddot{x}+b_{x x} \dot{x}+a_{x x} x+\left(b_{y x}+\frac{\lambda}{\bar{W}}\right) \dot{y}+a_{x y} y=0, \\
& \frac{1}{\bar{W}} \ddot{y}+b_{y y} \dot{y}+a_{y y} y+\left(b_{y x}-\frac{\lambda}{\bar{W}}\right) \dot{x}+a_{y x} x=0 .
\end{aligned}
$$

Up till now the reference force, $F_{0}$, has still not been chosen. A natural choice may be arrived at as follows. From the dimensionless pressure, $p$, the reactional force, for instance $F_{x}$, can be determined with

$$
F_{x}=\iint p P_{0} \cos \beta \mathrm{d} \Phi \mathrm{d} \Psi,
$$

in which $P_{0}$ (cf. sec. 5.3.1) is the reference pressure, $\Phi$ and $\Psi$ (cf. fig. 6.1) are the film coordinates, and $\beta$ is the angle of the film element with respect to the $X$-axis. Introducing dimensionless quantities we may write

$$
f_{x}=\iint \frac{\rho P_{0} R^{2}}{F_{0}} \cos \beta \mathrm{d} \varphi \mathrm{d} \psi=\iint p \cos \beta \mathrm{d} \varphi \mathrm{d} \psi,
$$

with

$$
\varphi=\frac{\Phi}{R}, \quad \psi=\frac{\Psi}{R}
$$

and

$$
F_{0}=P_{0} R^{2}=\frac{12 \eta \Omega R^{4}}{(\Delta R)^{2}}
$$

(with reference parameters: $H_{0}=\Delta R, L_{0}=R$ ). For many boundary conditions (which will not be discussed here), $p$, and thus $f_{x}$, depends only on the bearing geometry, journal position, and journal velocities; this means that $a_{x x}$, $b_{x x}$, etc., depend only on the (equilibrium) position of the journal.

Note. The above discussion, based on the "ordinary" Reynolds equation (5.12), will proceed analogously for the "generalised" equation (5.46). 
An alternative to the above choice of $F_{0}$ is the load capacity, $W$ ( $W=$ $\left.=F=\left(F_{x}{ }^{2}+F_{y}{ }^{2}\right)^{1 / 2}\right)$. This alternative is often encountered in the literature. An objection to this is the fact that $W$ becomes zero when the bearing is unloaded. In the present chapter, to facilitate a comparison with the literature, we shall use $F_{0}=W$ and distinguish the stability parameter $(\bar{W})$ based on this choise by the index $w$, i.e. $\bar{W}_{w}$. In subsequent chapters, however, we shall make use of (2.14).

Substitution of $x=x_{0} \exp (s \tau), y=y_{0} \exp (s \tau)$ in (2.13) yields the characteristic equation

$$
\begin{aligned}
& \left|\begin{array}{l}
\frac{1}{W} s^{2}+b_{x x} s+a_{x x}\left(b_{x y}+\frac{\lambda}{W}\right) s+a_{x y} \\
\left(b_{y x}-\frac{\lambda}{W}\right) s+a_{y x} \frac{1}{W} s^{2}+b_{y y} s+a_{y y}
\end{array}\right|= \\
& =\frac{1}{\bar{W}^{2}}\left(a_{0} s^{4}+a_{1} s^{3}+a_{2} s^{2}+a_{3} s+a_{4}\right)=0,
\end{aligned}
$$

in which

$$
\begin{aligned}
& a_{0}=1, \\
& a_{1}=\left(b_{x x}+b_{y y}\right) \bar{W} \\
& a_{2}=\left(a_{x x}+a_{y y}\right) \bar{W}+\left(b_{x x} b_{y y}-b_{x y} b_{y x}\right) \bar{W}^{2}+\left(b_{x y}-b_{y x}\right) \lambda \bar{W}+\lambda^{2}, \\
& a_{3}=\left(b_{x x} a_{y y}+b_{y y} a_{x x}-a_{x y} b_{y x}-a_{y x} b_{x y}\right) \bar{W}^{2}+\left(a_{x y}-a_{y x}\right) \bar{W} \lambda, \\
& a_{4}=\left(a_{x x} a_{y y}-a_{x y} a_{y x}\right) \bar{W}^{2} .
\end{aligned}
$$

The motion is stable if for all the roots, $s_{k}$, of the characteristic equation we can write:

$$
\operatorname{Re}\left(s_{k}\right)<0 \quad(k=1,2,3,4) .
$$

For (2.17) it is necessary (but not sufficient) that

$$
a_{j}>0 \quad(j=0,1,2,3,4) .
$$

According to the Routh-Hurwitz stability criterion ${ }^{2-6}$ ) it is necessary and sufficient for (2.17) that

$$
\begin{aligned}
& a_{0}>0 \text {, } \\
& a_{1}>0 \text {, } \\
& \left|\begin{array}{ll}
a_{1} & a_{0} \\
a_{3} & a_{2}
\end{array}\right|=a_{1} a_{2}-a_{0} a_{3}>0, \\
& \left|\begin{array}{lll}
a_{1} & a_{0} & 0 \\
a_{3} & a_{2} & a_{1} \\
0 & a_{4} & a_{3}
\end{array}\right|=a_{3}\left(a_{1} a_{2}-a_{0} a_{3}\right)-a_{1}^{2} a_{4}>0, \\
& a_{4}>0 \text {. }
\end{aligned}
$$


With (2.18) it is possible to reduce (2.19) to

$$
a_{0}>0, \quad a_{1}>0, \quad a_{2}>0, \quad a_{3}>0, \quad a_{4}>0
$$

and

$$
a_{3}\left(a_{1} a_{2}-a_{0} a_{3}\right)-a_{1}^{2} a_{4}>0 .
$$

If the coefficients of the bearing response for a certain equilibrium position are known, then we are able to examine by means of (2.20) whether a certain value of the stability parameter $\bar{W}$ (or $\bar{W}_{w}$ ) results in a stable or an unstable behaviour. Such scanning of the field in which $W$ is of practical value, leads for a number of equilibrium positions to a stability diagram in which the stable and unstable areas are partitioned by "stability-transition curves".

\subsubsection{Application to a rotor with smooth journal bearings}

From the literature the response coefficients of smooth journal bearings are known (Orcutt and Arwas ${ }^{2-4}$ )). These coefficients are recorded in figs 2.3 and 2.4. The system $X, Y, Z$ is chosen in such a way that the $X$-axis is parallel to the direction of the bearing load, $W$ (in the remaining chapters along $\mathbf{E}$ ).

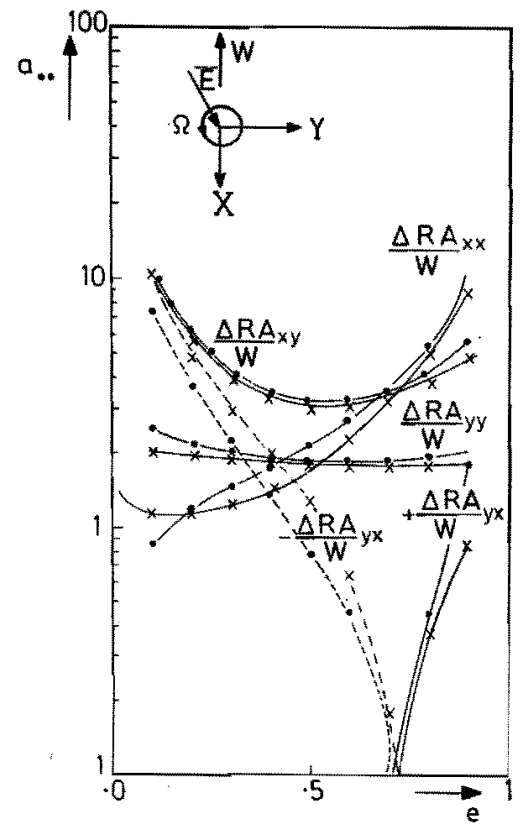

Fig. 2.3. Dimensionless stiffness coefficients of smooth journal bearing $(B / D=$ 1). _... $\times-$ Results of Orcutt and Arwas; __. ALG bearing calculated with the FEM.

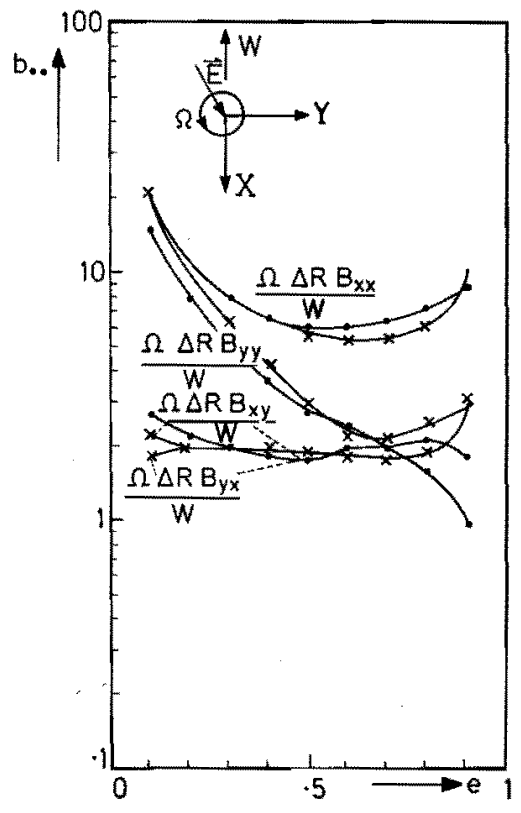

Fig. 2.4. Dimensionless damping coefficients of a smooth journal bearing $(B / D=1) .=\times \cdots$ Results of Orcutt and Arwas; _........ ALG bearing calculated with the FEM. 


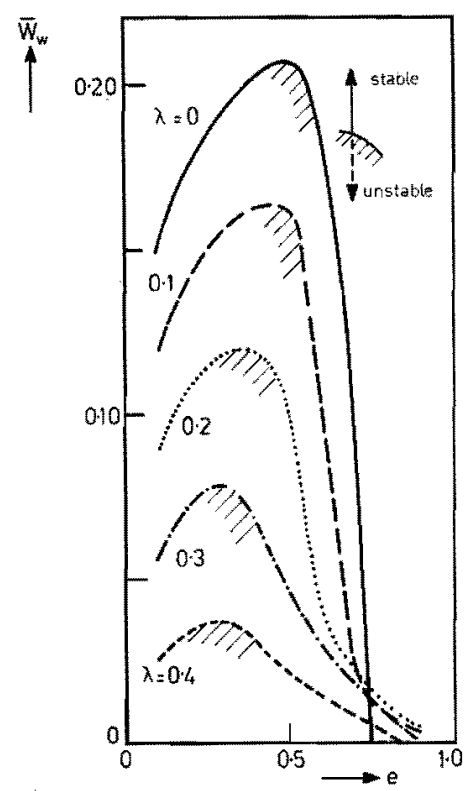

Fig. 2.5. Stability curves of a smooth journal bearing $(B / D=1)$ with a static load $W$. $\bar{W}_{w}=\frac{2 W}{M_{R} \Delta R \Omega^{2}}$ for translation $(\lambda=0)$ and $\bar{W}_{w}=\frac{W L^{2}}{2 J \Delta R \Omega^{2}}$ for conical motion $\left(\lambda=\frac{I}{J}\right)$.

Because the more accurate data of chapters 6 and 7 did not become available until later, the data of Orcutt and Arwas ${ }^{2-4}$ ) were used to calculate the stability curves of fig. 2.5. In a subsequent comparison of these data with the results of an ALG bearing (cf. chapter 7) the differences (figs 2.3 and 2.4) were small enough to dispense with a new calculation.

In accordance with the above theory, fig. 2.5 applies to both modes of motion: the curves $\lambda=0.1$ to 0.4 relate to a conical motion and the curve $\lambda=0$ refers to a conical motion without gyroscopic effects as well as to a translation. The parameter $e$ is the bearing eccentricity, and $e=|\mathbf{E}| / \Delta R$.

For the case $\lambda=0$ a comparison with data from the literature is possible ${ }^{2-1,2}$ ). Considering the fact that the bearing coefficients there used were slightly different, we can say that there is an excellent agreement between those data and the present curve.

It will be seen from fig. 2.5 that in the range $0<e<$ ca 0.74 an increase in the gyroscopic effect $(\lambda)$ improves the stability of the conical motion. Neglecting the gyroscopic effect (taking $\lambda \approx 0$ ), as many authors do, gives too pessimistic a picture of the rotor stability. The calculation proved that for $\lambda>$ ca 0.5 the stabilizing effect of the gyroscopic action was so strong that the stability curves came to lie below the $e$-axis, and hence the conical motion became inherently stable. Very remarkable is the range of ca $0.74<e<1$. 
It is common practice, and justified according to fig. 2.5 for the translational and conical mode at $\lambda=0$, to assume that a smooth cylindrical bearing is inherently stable in this range. This, however, is found to be invalid for the conical mode at $0<\lambda<\mathrm{ca} 0.5$, where instabilities can in fact occur. Since it is likely that, for example, some turbines operate in this range, instability might unexpectedly occur precisely because of gyroscopic effects. In order to gain a better insight into what takes place in this range, a simulation was performed with an analog computer. This aspect will not be theated here. Suffice it to say that the phenomenon could be accounted for by the fact that the gyroscopic effects influenced the mode of motion of the bearings in such a way that energy could be taken from the lubricant film, which would have given rise to instability.

\subsection{Stability of a symmetric rotor-and-bearing system with a constant bearing load rotating synchronously with the shaft}

\subsubsection{Equations of motion}

In sec. 2.2 we have examined the stability of a system in which the bearings support a static load. Another case encountered in practice is that of a load of constant magnitude rotating with the rotor, for instance an unbalanced rotor. If we assume once more that the load is symmetrical with respect to aa' (cf. fig. 2.1) and that the bearing response is rotationally symmetrical, then the vectors $\mathbf{E}$, denoting the equilibrium "position", will describe circles.

It appears to be convenient to relate, as it were, the system $X, Y, Z$ to the vectors $\mathbf{E}$ so that this system rotates with angular velocity $\Omega$ about the $Z$-axis. To find the equations of motion in $X, Y$ coordinates we may proceed as follows.

For the translational mode the equation of motion in the inertial space can again be expressed by

$$
\Delta \mathbf{F}=\frac{M_{R}}{2} \frac{\mathrm{d}^{2} \mathbf{X}}{\mathrm{d} t^{2}}
$$

With $\mathbf{e}_{j}$ representing the base vectors of the $X, Y, Z$ system, and with

$$
\mathbf{X}=X^{j} \mathbf{e}_{j}, \quad \frac{\mathrm{de}_{j}}{\mathrm{~d} t}=\boldsymbol{\Omega} \times \mathbf{e}_{j},
$$

we have

$$
\Delta \mathbf{F}=\frac{M_{R}}{2}\left(\frac{\mathrm{d}^{2} X^{j}}{\mathrm{~d} t^{2}} \mathbf{e}_{j}+2 \frac{\mathrm{d} X^{j}}{\mathrm{~d} t}\left(\boldsymbol{\Omega} \times \mathbf{e}_{j}\right)+X^{j}\left(\boldsymbol{\Omega} \times\left(\boldsymbol{\Omega} \times \mathbf{e}_{j}\right)\right)\right) .
$$

The vectors of (2.22) are still defined in the inertial space; in a space in which the system $X, Y, Z$ is stationary, the equation of motion becomes 


$$
\Delta \mathbf{F}=\frac{M_{R}}{2}\left(\frac{\mathrm{d}^{2} \mathbf{X}}{\mathrm{d} t^{2}}+2 \boldsymbol{\Omega} \times \frac{\mathrm{d} \mathbf{X}}{\mathrm{d} t}-\Omega^{2} \mathbf{X}\right)
$$

with $\Omega=|\Omega|$. Indicating the vectors of $(2.23)$ by their components with respect to the $X, Y, Z$ system we may write

$$
\begin{aligned}
& \Delta F_{x}=\frac{M_{R}}{2}\left(\frac{\mathrm{d}^{2} X}{\mathrm{~d} t^{2}}-2 \Omega \frac{\mathrm{d} Y}{\mathrm{~d} t}-\Omega^{2} X\right) \\
& \Delta F_{y}=\frac{M_{R}}{2}\left(\frac{\mathrm{d}^{2} Y}{\mathrm{~d} t^{2}}+2 \Omega \frac{\mathrm{d} X}{\mathrm{~d} t}-\Omega^{2} Y\right) .
\end{aligned}
$$

For the conical mode the equation of motion in a space in which the $U, V, W$ system (defined with respect to $X, Y, Z$ in the same way as in sec. 2.2.1) is stationary, becomes once more

$$
\mathbf{T}=\frac{\mathrm{d} \mathbf{H}}{\mathrm{d} t}+\left(\boldsymbol{\Omega}_{r}+\boldsymbol{\Omega}\right) \times \mathbf{H} .
$$

For $\theta \ll 1$ we have

$$
\begin{aligned}
& T_{u} \approx-\Delta F_{y} L \\
& T_{v} \approx+\Delta F_{x} L \\
& T_{w} \approx 0 ; \\
& \Omega_{r u}+\Omega_{u} \approx \frac{2}{L}\left(-\frac{\mathrm{d} Y}{\mathrm{~d} t}-\Omega X\right), \\
& \Omega_{r v}+\Omega_{v} \approx \frac{2}{L}\left(+\frac{\mathrm{d} X}{\mathrm{~d} t}-\Omega Y\right) \\
& \Omega_{r w}+\Omega_{w} \approx \Omega \\
& H_{u}=J\left(\Omega_{r u}+\Omega_{w}\right), \\
& H_{v}=J\left(\Omega_{r v}+\Omega_{v}\right), \\
& H_{w}=I\left(\Omega_{r w}+\Omega_{w}\right) .
\end{aligned}
$$

From $(2.25),(2.26),(2.27)$ and $(2.28)$ it follows that

$$
\begin{aligned}
& \Delta F_{x}=\frac{2 J}{L^{2}}\left(\frac{\mathrm{d}^{2} X}{\mathrm{~d} t^{2}}-(2-\lambda) \Omega \frac{\mathrm{d} Y}{\mathrm{~d} t}-(1-\lambda) \Omega^{2} X\right) \\
& \Delta F_{y}=\frac{2 J}{L^{2}}\left(\frac{\mathrm{d}^{2} Y}{\mathrm{~d} t^{2}}+(2-\lambda) \Omega \frac{\mathrm{d} X}{\mathrm{~d} t}-(1-\lambda) \Omega^{2} Y\right) .
\end{aligned}
$$


Using the given definitions of $\bar{W}$ and $\lambda$ and introducing dimensionless quantities we may write, instead of (2.24) and (2.29),

$$
\begin{aligned}
& \Delta f_{x}=\frac{1}{\bar{W}}[\ddot{x}-(2-\lambda) \dot{y}-(1-\lambda) x], \\
& \Delta f_{y}=\frac{1}{\bar{W}}[\ddot{y}+(2-\lambda) \dot{x}-(1-\lambda) y] .
\end{aligned}
$$

Assuming again that the relations (2.12b) may be used, we obtain

$$
\begin{aligned}
& \frac{1}{W} \ddot{x}+b_{x x} \dot{x}+\left(a_{x x}-\frac{1-\lambda}{W}\right) x+\left(b_{x y}-\frac{2-\lambda}{W}\right) \dot{y}+a_{x y} y=0, \\
& \frac{1}{W} \ddot{y}+b_{y y} \dot{y}+\left(a_{y y}-\frac{1-\lambda}{\bar{W}}\right) y+\left(b_{y x}+\frac{2-\lambda}{\bar{W}}\right) \dot{x}+a_{y x} x=0 .
\end{aligned}
$$

Equations (2.31) correspond to eqs (2.13): the characteristic equation can be found in the same way as with (2.13).

\subsubsection{Application to a rotor with smooth journal bearings}

The response coefficients of (2.31) are defined in the rotating system $X, Y, Z$, and should normally be calculated in this system. With a smooth journal bearing, however, it is possible to deduce the coefficients in the rotating system directly from the coefficients that apply in the stationary system of sec. 2.2.2. Let us regard fig. 2.6 and suppose that we know the coefficients in fig. 2.6a.

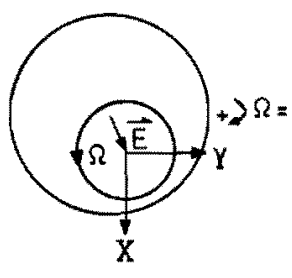

a)

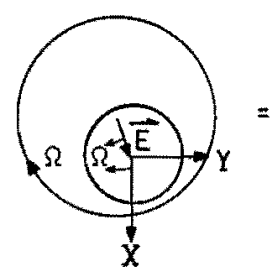

b)

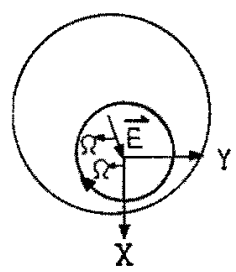

c)

Fig. 2.6. Transformation of a statically loaded bearing into a bearing with a rotating load.

It is permissible to superimpose an angular velocity $\Omega$ on fig. $2.6 a$, without the flow in the lubricant being affected, and hence the coefficients are unaffected. For smooth bearings it is of no consequence (with the usual assumptions on film flow) whether the bush or the shaft rotates, so that the situation in fig. $2.6 \mathrm{c}$ is identical with that in fig. 2.6b. In fig. $2.6 \mathrm{c} \Omega$ is negative with respect to the $X, Y$ coordinates. Reversing of the direction of the $Y$-axis leads to a positive $\Omega$ (assumed in sec. 2.3.1). This implies that the coeficients in the rotating system (indicated by the index rot) can be found from the coefficients in the stationary system (no index) in the following way: 


$$
\begin{array}{ll}
a_{x x \text { rot }}=a_{x x}, & b_{x x \text { rot }}=b_{x x}, \\
a_{x y \text { rot }}=-a_{x y y} & b_{x y \text { rot }}=-b_{x y}, \\
a_{y x \text { rot }}=-a_{y x}, & b_{y x \text { rot }}=-b_{y x} \\
a_{y y \text { rot }}=a_{y y}, & b_{y y \text { rot }}=b_{y y} .
\end{array}
$$

The stability-transition curves calculated for smooth journal bearings are recorded in fig. 2.7. Here again an increase of the gyroscopic effect, and hence an increase in $\lambda$, turns out to have a stabilising effect on the conical motion. Unlike the conclusions which can be drawn from fig. 2.5, the conical motion and the translation are not inherently stable in the range $e>c a 0.7$ and $\lambda=0$. Only for $\lambda>$ ca 0.6 does the conical motion become inherently stable.

For eccentricities of $e<\mathrm{ca} .0 .7$ the value of $\widetilde{W}_{w}$, defined by a stability curve, increases but slowly, or decreases, with an increase in $e$. But because $W$ increases rapidly with $e$, the net effect of an increase in $e$ (or in $W$ ) on a given rotor-and-bearing system will be an increase in the speed at which instability sets in. This is equivalent to saying that in this case an increase in the unbalance of the rotor has a stabilising effect.

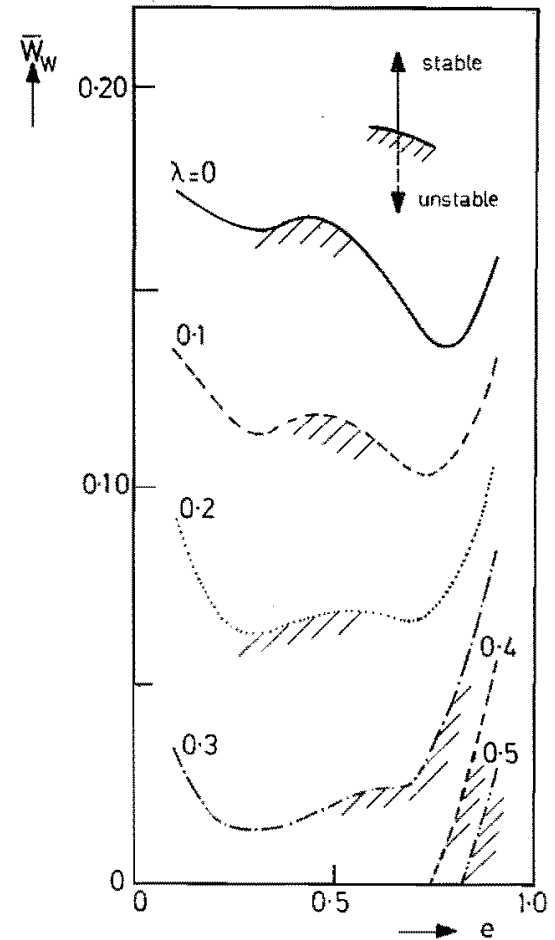

Fig. 2.7. Stability curves of a smooth journal bearing $(B / D=1)$ with a load of constant magnitude, $W$, rotating with the angular speed of the rotor. $\bar{W}_{w}=\frac{2 W}{M_{R} \Delta R \Omega^{2}}$ for translation $(\lambda=0)$ and $W_{w}=\frac{W L^{2}}{2 J \Delta R \Omega^{2}}$ for conical motion $\left(\lambda=\frac{1}{J}\right)$ 


\section{REFERENCES}

$\left.{ }^{2-1}\right)$ B. Sternlicht and N. F. Rieger, Rotor stability, Conference Lub. and Wear, London (September 1967) 182, part 3A, paper 7.

$\left.{ }^{2-2}\right)$ R. Holmes, Instability phenomena due to circular bearing oil films, J. mech. Eng. 8, 419-425, 1966.

2-3) J. W. Lund, The stability of an elastic rotor in journal bearings with flexible, damped supports, Trans. ASME, J. appl. Mech. 32, 911-920, 1965.

2-4) F. K. Orcutt and E. B. A rwas, The steady-state and dynamic characteristics of a full circular bearing and a partial arc bearing in the laminar and turbulent flow regimes, Trans. ASME, J. Lub. Techn. 89, 143-153, 1967.

2-5) J. P. Reinhoudt, A grease-lubricated hydrodynamic bearing system for a satellite flywheel, ASLE-ASME Lub. Conference, Houston, Texas (October 1969);

J. Lub. Eng. 26, no. 3, paper 22, March 1970.

2-6) J. G. Malkin, Theorie der Stabilität einer Bewegung, R. Oldenburg, München, 1959. 


\section{LINEAR AND NONLINEAR ASPECTS OF THE STABILITY OF A SYMMETRIC ROTOR-AND-BEARING SYSTEM WITH RIGID BEARING SUPPORTS AND ROTATIONALLY SYMMETRIC BEARING RESPONSE}

\subsection{Introduction}

Let us now consider the motion, and in particular the stability, of the symmetric "standard" rotor (fig. 2.1) for the special case that the bearing response is rotationally symmetric with respect to the $Z$-axis of the $X, Y, Z$ system shown in fig. 2.1. Such a situation occurs, for instance, with an unloaded bearing having a rotationally symmetric geometry. The concentric position of the shaft, then, is the equilibrium position, and a rotation of the $X, Y, Z$ system about the $Z$ axis does not affect the response coefficients.

The rotational symmetry in a bearing simplifies the investigation of the motion of the rotor. This means, for one thing, that it is possible to derive stability criteria which can be easily applied in practice, and for another that an insight can be gained into the behaviour at bearing displacements that are so large that linearisation of the bearing response is no longer permissible.

Large bearing displacements occur, for example, when, on the basis of the linear stability theory, a rotor becomes unstable. Some authors express the expectation that nonlinear effects may ensure that the bearing displacements do not become steadily larger but remain limited. With nonlinear effects taken into account, our analysis shows that for a rotationally symmetric response the bearing displacement will - under certain conditions - remain within bounds.

\subsection{Types of bearings with rotationally symmetric response}

A bearing with rotationally symmetric response will be considered to be a bearing in which a rotation of the coordinate system $X, Y, Z$ (cf. fig. 2.1) through an arbitrary angle about the $Z$-axis does not affect the response coefficients. In a number of cases a bearing appears to have a rotationally symmetric response.

\section{Case 1}

A bearing which has a rotationally symmetric geometry with respect to the $Z$-axis has a rotationally symmetric response, too. Examples: unloaded smooth and helically grooved journal bearings and spherical spiral-groove bearings without radial load.

On the assumption that linearisation is permissible the general relations (2.12b) are still true, but in the case of rotational symmetry it can be proved that the following relations should exist between the response coefficients:

$$
a_{x x}=a_{y y} \quad b_{x x}=b_{y y}, \quad a_{x y}=-a_{y x}, \quad b_{x y}=-b_{y x} .
$$


Introducing the coefficients of rotationally symmetric response,

$$
\begin{aligned}
a & =a_{x x}=a_{y y}, \\
b & =b_{x x}=b_{y y}, \\
\delta_{c} b & =a_{x y}=-a_{y x}, \\
b_{t} & =b_{x y}=-b_{y x},
\end{aligned}
$$

we may write, instead of (2.12b):

$$
\begin{aligned}
& \Delta f_{x}=-a x-b \dot{x}-\delta_{c} b y-b_{t} \dot{y}, \\
& \Delta f_{y}=-a y-b \dot{y}+\delta_{c} b x+b_{t} \dot{x} .
\end{aligned}
$$

With respect to the parameter $\delta_{c}$ it may be noted that, for a circular orbit about the equilibrium position, $\delta_{c}$ is the dimensionless angular velocity at which the bearing response is exactly radial. For this reason we shall call $\delta_{c}$ the characteristic angular velocity of the bearing. With smooth bearings it can easily be proved that $\delta_{c}=\frac{1}{2}$; for the majority of other bearing types $\delta_{c} \approx \frac{1}{2}$.

\section{Case 2}

A bearing with three or more identical parts that are equally spaced circumferentially (cf. fig. 3.1) possesses (at the "concentric" position) a rotationally
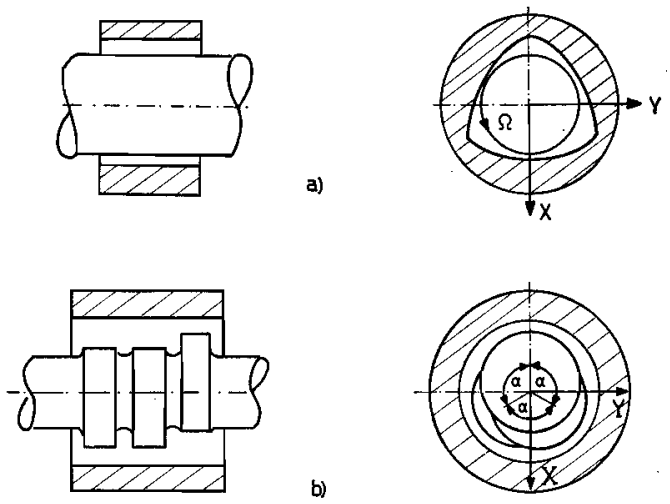

b)
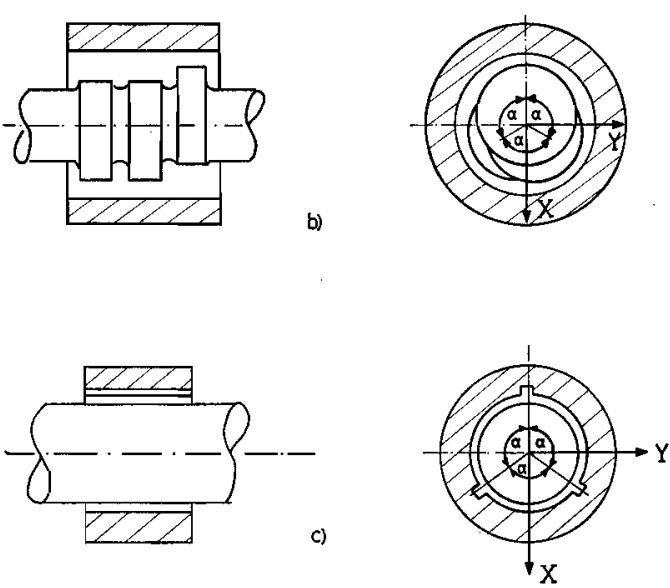

Fig. 3.1. Bearings with rotationally symmetric response in the concentric position. (a) Threelobed bearing; $(b)$ bearing with three identical parts; $(c)$ smooth bearing with equally spaced oil grooves. 
symmetric response to deflections that still allow of linearisation (cf. eq. (2.12b)). If, for one part, the relation $(2.12 b)$ is true, then, for the combination of all parts, the relation (3.3) applies and it can be proved that

$$
\begin{aligned}
a & =\frac{n}{2}\left(a_{x x}+\dot{a}_{y y}\right), \\
b & =\frac{n}{2}\left(b_{x x}+b_{y y}\right), \\
\delta_{c} b & =\frac{n}{2}\left(a_{x y}-a_{y x}\right), \\
b_{t} & =\frac{n}{2}\left(b_{x y}-b_{y x}\right),
\end{aligned}
$$

with $n=$ number of parts.

Case 3

Various grooved bearings appear to have a rotationally symmetric response. Examples are the helical-groove bearing (ORS bearing) and the spherical spiralgroove bearings (SOAT and SORS bearings), which will be considered in chapter 7. With these bearings the rotational symmetry is not limited to the concentric position but is maintained up to relatively high eccentricities. Indeed, the symmetry is not exact and the relations (3.1) are only approximately true, but the approximation appears to be admissible in many practical applications. An averaging of the response coefficients will make a (rotationally symmetric) calculation of these bearings more accurate:

$$
\begin{aligned}
a & =\frac{1}{2}\left(a_{x x}+a_{y y}\right), \\
b & =\frac{1}{2}\left(b_{x x}+b_{y y}\right), \\
\delta_{c} b & =\frac{1}{2}\left(a_{x y}-a_{y x}\right), \\
b_{t} & =\frac{1}{2}\left(b_{x y}-b_{y x}\right),
\end{aligned}
$$

3.3. A stability criterion of the case of rotationally symmetric response

Analogously to (2.13) the equations of motion become

$$
\begin{aligned}
& \frac{1}{\bar{W}} \ddot{x}+b \dot{x}+a x+\left(b_{t}+\frac{\lambda}{W}\right) \dot{y}+\delta_{c} b y=0, \\
& \frac{1}{\bar{W}} \ddot{y}+b \dot{y}+a y-\left(b_{t}+\frac{\lambda}{\bar{W}}\right) \dot{x}-\delta_{c} b x=0 .
\end{aligned}
$$


The characteristic determinant and characteristic equation (cf. eq. (2.15)) are

$$
\begin{aligned}
& \left|\begin{array}{cc}
\frac{1}{W} s^{2}+b s+a & \left(b_{t}+\frac{\lambda}{W}\right) s+\delta_{c} b \\
-\left(b_{t}+\frac{\lambda}{W}\right) s-\delta_{c} b & \frac{1}{W} s^{2}+b s+a
\end{array}\right|= \\
& =\frac{1}{\bar{W}^{2}}\left(a_{0} s^{4}+a_{1} s^{3}+a_{2} s^{2}+a_{3} s+a_{4}\right)=0,
\end{aligned}
$$

in which

$$
\begin{aligned}
& a_{0}=1, \\
& a_{1}=2 b \bar{W} \\
& a_{2}=2 a \bar{W}+\left(b^{2}+b_{t}^{2}\right) \bar{W}^{2}+2 b_{t} \lambda \bar{W}+\lambda^{2}, \\
& a_{3}=2 b \bar{W}\left(a \bar{W}+\delta_{c} b_{t} \bar{W}+\delta_{c} \lambda\right), \\
& a_{4}=\left(a^{2}+\delta_{c}^{2} b^{2}\right) \bar{W}^{2} .
\end{aligned}
$$

For stability the conditions (2.19) or (2.20) should be satisfied. If we postulate that the damping coefficient, $b$, the stiffness coefficient, $a$, and the characteristic velocity, $\delta_{c}$, are positive, and further that $\delta_{c} b_{t}<a$ (this is found to be true for all the bearings that have been investigated), then the only condition that must still be met is

$$
a_{3}\left(a_{1} a_{2}-a_{0} a_{3}\right)-a_{1}^{2} a_{4}>0 .
$$

When this is worked out, it is found that the motion will be stable if the following condition is satisfied:

$$
\left(a+\delta_{c} b_{t}\right)-\frac{\delta_{c}^{2}}{\bar{W}}\left(1-\frac{\lambda}{\delta_{c}}\right)>0
$$

\section{Example}

The response coefficients of an unloaded bearing with optimal radial stiffness, known as an ORS bearing, are (cf. sec. 7.4.4)

$$
a \approx 0.20, \quad b \approx 0.59, \quad \delta_{c} b \approx 0.31, \quad b_{t} \approx 0 .
$$

According to $(3.10)$ the translational mode $(\lambda=0)$ will be stable if

$$
\bar{W}>\frac{\delta_{c}{ }^{2}\left(1-\lambda / \delta_{c}\right)}{a+\delta_{c} b_{t}}=1 \cdot 35 .
$$

This result corresponds to the result found (in another way) in fig. 7.18. For a given rotor, with $\eta=0.03 \mathrm{Ns} / \mathrm{m}^{2}, R=5.10^{-3} \mathrm{~m}, \Delta R=20.10^{-6} \mathrm{~m}$, $M_{\mathrm{R}}=100 \mathrm{~kg}$, and (cf. eq. (2.14)) 


$$
\begin{gathered}
-26- \\
W=\frac{2 F_{0}}{M_{R} \Delta R \Omega^{2}}=\frac{24 \eta R^{4}}{M_{R}(\Delta R)^{3} \Omega}
\end{gathered}
$$

the translational mode is stable if the angular speed satisfies the condition

$$
\Omega<415 \mathrm{rad} / \mathrm{s} \text {. }
$$

The translation will be unstable if the speed exceeds ca $4000 \mathrm{rpm}$.

\section{Remarks}

(1) In an unloaded smooth journal bearing we have $a=b_{t}=0$. If in addition $\lambda=0$, then (3.10) predicts that the bearing will be inherently unstable; in the presence of a gyroscopic effect the conical motion of the rotor is only stable with $\lambda>\delta_{c}$. Because of $\delta_{c}=0.5$ the motion will then be stable with $\lambda>0.5$.

(2) For many bearings with rotationally symmetric response (chapter 7) it is true that $b_{t} \approx 0, \delta_{c} \approx 0.5$; if we then assume $\lambda=0$, the motion will be stable if $a>$ ca $1 / 4 \bar{W}$.

(3) Of major importance is the conclusion that for stability the radial bearing stiffness at the characteristic velocity, $\delta_{c}$, plays the leading part.

\subsection{Physical interpretation}

\subsubsection{Physical meaning of the parameters}

In this section we shall introduce a physical model to enhance our insight into the behaviour of a rotor-and-beating system at the transition of stability. That model should explain that at this transition (cf. eq. (3.10))

$$
\left(a+\delta_{c} b_{t}\right)-\frac{\delta_{c}{ }^{2}}{\bar{W}}+\frac{\delta_{c} \lambda}{W}=0 .
$$

In our model it is assumed that, at the transition of stability, the journals circulate about their equilibrium position, that the bearing response is in equilibrium with the centrifugal and gyroscopic forces, and that the parameters in (3.10) may be interpreted as follows:

1

$\bar{W}$

$\delta_{c}$

$a+\delta_{c} b_{t}$

$\frac{\delta_{c}^{2}}{\bar{W}}$

$\frac{\delta_{c} \lambda}{\bar{W}}$ is the dimensionless rotor mass, is the dimensionless angular velocity, is the radial stiffness at the velocity $\delta_{c}$, is the stiffness induced by the centrifugal forces, is the stiffness induced by the gyroscopic forces. 
The left-hand side of (3.11) represents an effective stiffness: if this effective stiffness is negative, the motion is unstable, and if it is positive, then the motion is stable. At the transition of stability the effective stiffness is exactly zero.

In the next section the motion will be described in terms of polar coordinates and it will be shown that the proposed interpretation is correct.

\subsubsection{Equations of motion in polar coordinates}

According to (2.11) the dimensionless equations of motion expressed in Cartesian coordinates read:

$$
\begin{aligned}
& \Delta f_{x}=\frac{1}{\bar{W}}(\ddot{x}+\lambda \dot{y}), \\
& \Delta f_{y}=\frac{1}{\bar{W}}(\ddot{y}-\lambda \dot{x}) .
\end{aligned}
$$

For the transformation into polar coordinates (see fig. 3.2) we make use of

$$
\begin{gathered}
x=e \cos \alpha, \quad y=e \sin \alpha, \quad w=\frac{\mathrm{d} \alpha}{\mathrm{d} \tau}, \\
\Delta f_{x}=\Delta f_{r} \cos \alpha-\Delta f_{t} \sin \alpha, \\
\Delta f_{y}=\Delta f_{r} \sin \alpha+\Delta f_{t} \cos \alpha .
\end{gathered}
$$

We then find the equations in the following form:

$$
\begin{aligned}
& \frac{\mathrm{d}^{2} e}{\mathrm{~d} \tau^{2}}+\left(\lambda w-w^{2}\right) e-\bar{W} \Delta f_{r}=0, \\
& \frac{\mathrm{d} w}{\mathrm{~d} \tau}-\frac{\lambda-2 w}{e} \frac{\mathrm{d} e}{\mathrm{~d} \tau}-\bar{W} \frac{\Delta f_{t}}{e}=0 .
\end{aligned}
$$

Let us now investigate the circumstances in which a circular motion can exist. If we assume that the journal does in fact describe a circle, it must be true that

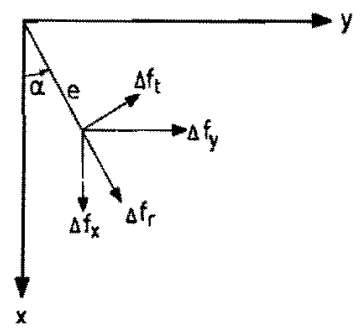

Fig. 3.2. Transformation into polar coordinates. 


$$
\frac{\mathrm{d}^{2} e}{\mathrm{~d} \tau^{2}}=\frac{\mathrm{d} e}{\mathrm{~d} \tau}=\frac{\mathrm{d} w}{\mathrm{~d} \tau}=0 \quad \text { and } \quad e>0
$$

In conjunction with (3.14b) this gives

$$
\Delta f_{t}=0
$$

From (3.3) it follows that

$$
\begin{aligned}
& \Delta f_{t}=-b e w+\delta_{c} b e+b_{t} \frac{\mathrm{d} e}{\mathrm{~d} \tau} \\
& \Delta f_{r}=-a e-b \frac{\mathrm{d} e}{\mathrm{~d} \tau}-b_{t} e w
\end{aligned}
$$

and from (3.15), (3.16) and (3.17a) we obtain

$$
w=\delta_{c}
$$

With (3.17a) we can rewrite (3.14a) as

$$
\frac{1}{W} \frac{\mathrm{d}^{2} e}{\mathrm{~d} \tau^{2}}+\frac{\lambda w-w^{2}}{W} e-\left(-a e-b \frac{\mathrm{d} e}{\mathrm{~d} \tau}-b_{z} e w\right)=0,
$$

and with (3.15) and (3.18)

$$
\left[\left(a+\delta_{c} b_{t}\right)-\frac{\delta_{c}^{2}}{\bar{W}}\left(1-\frac{\lambda}{\delta_{c}}\right)\right] e=0
$$

(3.15) and (3.19) imply that

$$
\left(a+\delta_{c} b_{t}\right)-\frac{\delta_{c}^{2}}{\bar{W}}+\frac{\delta_{c} \lambda}{\bar{W}}=0
$$

which is the same as (3.11), and therefore we may draw the conclusion that our model was correct and that it is indeed justifiable to attribute the proposed interpretation to (3.11).

\subsection{The effect of large bearing displacements}

\subsubsection{The possibility of a circular orbit}

If the displacements of the bearings are "large", then it is not necessarily permissible to linearise $\Delta f_{r}$ and $\Delta f_{t}$ (see (3.17)). Nevertheless it is interesting to ascertain whether the bearing displacements can remain limited even if, in virtue of the criterion expressed by (3.10), the motion is unstable. 
Guided by the results of the linear theory, let us examine whether for large displacements a circular orbit is possible and, if so, whether this motion is stable. We shall assume that the bearing response possess rotational symmetry also in the case of large displacements and that linearisation of the equations of motion (2.11) continues to be permissible (this is the case at all times with bearings having a rotationally symmetrical geometry; in the case of bearings with three or more lobes it is a meaningful approximation).

From the Reynolds differential equation, briefly alluded to in chapter 2 and derived in chapter 5 , it follows that with boundary conditions that are, at the most, functions of $e, \mathrm{~d} e / \mathrm{d} \tau, e w$, we may write:

$$
\begin{aligned}
& f_{r}=f_{r}\left(e, \frac{\mathrm{d} e}{\mathrm{~d} \tau}, e w\right), \\
& f_{t}=f_{t}\left(e, \frac{\mathrm{d} e}{\mathrm{~d} \tau}, e w\right) .
\end{aligned}
$$

Let us suppose that a circular motion is taking place; in accordance with (3.14a) and (3.20) the displacement $e_{0}$ and the angular velocity $w_{0}$ of this motion are then defined by the formulae

$$
\begin{gathered}
\left(\lambda w_{0}-w_{0}^{2}\right) e_{0}-\bar{W} f_{r}\left(e_{0}, 0, e_{0} w_{0}\right)=0, \\
\frac{\bar{W}}{e_{0}} f_{t}\left(e_{0}, 0, e_{0} w_{0}\right)=0
\end{gathered}
$$

( $f_{r}$ may no longer be linearised; for this reason the prefix $A$ is omitted). Because of $W / e_{0} \neq 0$, it follows from (3.21b) that

$$
f_{t}\left(e_{0}, 0, e_{0} w_{0}\right)=0 .
$$

This means that $w_{0}$ is equal to $\delta_{c}$, the characteristic angular velocity at which the direction of the bearing response is purely radial. Calculations of smooth and grooved journal bearings (see also chapter 7) show that $\delta_{c}$ is virtually independent of $e_{0}$ (almost invariably we find $\delta_{c} \approx 0.5$ ).

With $\delta_{c}$ known, $e_{0}$ can be found from (3.21a):

$$
-\frac{f_{r}}{e_{0}}\left(e_{0}, 0, e_{0} w_{0}\right)-\frac{\delta_{c}^{2}}{W}\left(1-\frac{\lambda}{\delta_{c}}\right)=0 .
$$

Note. Normally (3.23) has a solution, $e_{0}>0$, only if the motion is unstable in virtue of (3.10) and if the "stiffness" increases with $e_{0}$ (fig. 3.3), i.e.

$$
-\frac{f_{r}}{e_{0}}>a+\delta_{c} b_{t} .
$$




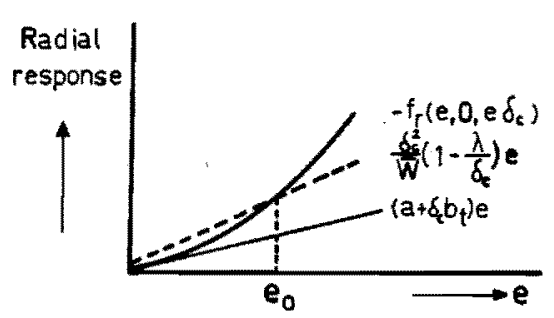

Fig. 3.3. The "normal shape" of $f_{r}\left(e, 0, e \delta_{c}\right)$.

\subsubsection{The stability of the circular orbit}

If from (3.21) a solution $\left(e_{0}, w_{0}\right)$ is found, then it will be necessary to investigate whether this solution implies stable motion. By studying slight motions about this "equilibrium" solution we are able to investigate its "stability".

Let, in (3.14),

$$
\begin{array}{lll}
e=e_{0}+\Delta e, & \text { with } & |\Delta e| \ll\left|e_{0}\right| \\
w=w_{0}+\Delta w, & \text { with } & |\Delta w| \ll\left|w_{0}\right| .
\end{array}
$$

This yields

$$
\begin{gathered}
\Delta \ddot{e}+\left(\lambda w_{0}-w_{0}{ }^{2}\right) \Delta e+\left(\lambda-2 w_{0}\right) e_{0} \Delta w+ \\
-\bar{W}\left(\frac{\partial f_{r}}{\partial e} \Delta e+\frac{\partial f_{r}}{\partial \dot{e}} \Delta \dot{e}+\frac{\partial f_{r}}{\partial e w} \Delta e w\right)=0, \\
e_{0} \Delta \dot{w}-\left(\lambda-2 w_{0}\right) \Delta \dot{e}+ \\
-W\left(\frac{\partial f_{t}}{\partial e} \Delta e+\frac{\partial f_{t}}{\partial \dot{e}} \Delta \dot{e}+\frac{\partial f_{t}}{\partial e w} \Delta e w\right)=0 .
\end{gathered}
$$

From calculations it is found that in general

$$
\left(\frac{\partial f_{r}}{\partial e w}\right)_{e_{0}, \delta_{c}},\left(\frac{\partial f_{t}}{\partial e}\right)_{e_{0}, \delta_{c}},\left(\frac{\partial f_{r}}{\partial \dot{e}}\right)_{e_{0}, \delta_{c}}
$$

are negligible with respect to the other terms. With

where

$$
\left(\frac{\partial f_{r}}{\partial e}\right)_{e_{0}, \delta_{c}}=-a_{e}, \quad\left(\frac{\partial f_{r}}{\partial \dot{e}}\right)_{e_{0}, \delta_{c}}=-b_{e}, \quad\left(\frac{\partial f_{t}}{\partial e w}\right)_{e_{0}, \delta_{c}}=-b_{f},
$$

one obtains

$$
a_{e}>0, \quad b_{e}>0, \quad b_{f}>0,
$$

$$
\begin{aligned}
& \Delta \ddot{e}+\left(\lambda w_{0}-w_{0}^{2}\right) \Delta e+\left(\lambda-2 w_{0}\right) e_{0} \Delta w+\bar{W}\left(a_{e} \Delta e+b_{e} \Delta \dot{e}\right)=0, \\
& e_{0} \Delta \dot{w}-\left(\lambda-2 w_{0}\right) \Delta \dot{e}+W b_{f} e_{0} \Delta w=0
\end{aligned}
$$


With $p=\bar{W} a_{\mathrm{e}}+\left(\lambda w_{0}-w_{0}{ }^{2}\right)$, the characteristic determinant and the characteristic equation become:

$$
\begin{gathered}
\left|\begin{array}{cc}
s^{2}+\breve{W} b_{e} s+p & \left(\lambda-2 w_{0}\right) \\
-\left(\lambda-2 w_{0}\right) s & s+W b_{f}
\end{array}\right|=s^{3}+a_{1} s^{2}+a_{2} s+a_{3}=0 \\
a_{1}=\bar{W}\left(b_{e}+b_{f}\right) \\
a_{2}=W^{2} b_{e} b_{f}+\left(\lambda-2 w_{0}\right)^{2}+p \\
a_{3}=\bar{W} p b_{f}
\end{gathered}
$$

For stability the following relations should hold:

$a_{1}>0$, which is satisfied because $W>0, b_{e}>0, b_{f}>0$;

$a_{3}>0$, which is satisfied because $p>0$;

$a_{1} a_{2}-a_{3}>0$, in other words,

$b_{e}\left[\bar{W}^{2} b_{e} b_{f}+\left(\lambda-2 w_{0}\right)^{2}+p\right]+b_{f}\left[\bar{W}^{2} b_{e} b_{f}+\left(\lambda-2 w_{0}\right)^{2}\right]>0$,

which is satisfied, provided $p>0$. From this and the definition given for $p$ it follows that the circular motion is stable if

$$
p=\bar{W} a_{e}+\left(\lambda w_{0}-w_{0}{ }^{2}\right)>0,
$$

or, in conjunction with (3.23) and $w_{0}=\delta_{c}$, if

$$
a_{e}>\frac{-f_{r}\left(e_{0}, 0, e_{0} \delta_{c}\right)}{e_{0}} .
$$

If (3.33) is satisfied, then the circular motion is stable.

Figure 3.4 shows examples of a stable and an unstable point of intersection.

Note. In the foregoing we have shown that a circular, stable motion is possible if (3.33) is satisfied. This does not, of course, form a rigid proof that no other motions can exist, although on the basis of experiments and of calculations with the aid of the analog computer it seems plausible to assume that the circular orbit is usually the only practical possibility.

\subsection{Conclusions and supplementary remarks}

The assumption of the rotationally symmetric bearing response simplifies the theory and provides a clear insight into the physical behaviour of a rotor at the boundary of stability.

A relatively large number of bearings exhibit a rotationally symmetric response and can be treated by means of the simple theory given above.

By including nonlinear effects in the theory, we were able to show that, under 

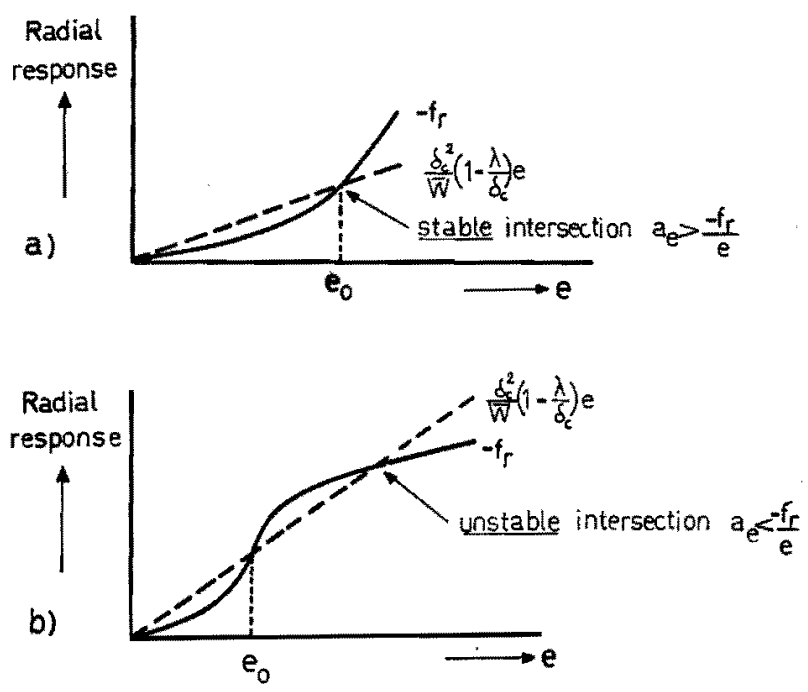

Fig. 3.4. (a) "Stable" and (b) "unstable" points of intersection.

certain other conditions, circularly stable orbits of the bearing could exist. If, on the other hand, the bearing were then considered in terms of the linear theory, then the motion would be found to be unstable.

On account of the "nonlinear" stability behaviour a bearing can yet function properly if it is considered to be unstable according to the linear theory. However, the journal of the bearing will vibrate to a greater or lesser extent, because the journal describes an orbit. But if such vibrations were acceptable, then it would be possible to design bearings with a far better "nonlinear" stability than could be attained on the basis of only the linear theory.

It is often very important to be able to treat bearings, which in themselves are rotationally asymmetric, as being rotationally symmetric by averaging the response coefficients; this permits of finding a practicable approximation of the stability parameter $\bar{W}$ by means of (3.10) and without the more complicated calculations of chapter 2 (see also the examples in chapter 7). 


\section{THE EFFECT OF FLEXIBLE BEARING SUPPORTS ON THE STABILITY OF A SYMMETRIC ROTOR-AND-BEARING SYSTEM}

\subsection{Introduction}

In the foregoing we have at all times assumed that the nonrotating part of the bearings is rigidly supported. This assumption is only permissible if the displacements of the support due to deflection are negligible with respect to those of the bearing itself. In a number of cases, however, the support of the bearing is so flexible that this should be taken into account in a consideration of the stability.

As compared with a rigid support, a flexible one can have both a destabilising and a stabilising effect. As a rule a decrease in stiffness of an undamped support gives rise to instabilities at lower speeds, so that it has a destabilising effect. But the provision of damping in a flexible support improves the stability of the rotor, sometimes even to such an extent that we may speak of a stabilising support. Figure 4.1 shows an example of such a stabilising support, viz. the O-ring suspension of the bearing bush in an aerostatic bearing. Kerr ${ }^{4-1}$ ) has published experimental results of air bearings supported by O-ring, from which the stabilising effect of the support becomes evident. Powell and Tempest ${ }^{4-3}$ ) reported that experimental dental turbines equipped with a support of this type reached speeds of as high as 720000 r.p.m.

Some publications on the theory of gas bearings also deal with the effect of the support on the stability. Lund ${ }^{4-5}$ ) has investigated the effect of a flexible support, with dampers, on the stability of a symmetric rotor, and he has applied the results to gas bearings. He employed the zero-point method, took the flexibility of the shaft into account in a somewhat questionable manner (cf. chapter 1 of the present work), and confines himself to supports having rotational symmetry. Marsh ${ }^{4-2}$ ) obtained the bearing coefficients by a linearising of the Reynolds equation, but then he proceeded in essentially the same manner as Lund, i.e. by using the zero-point method. Tondl ${ }^{4-6}$ ) investigated the effect

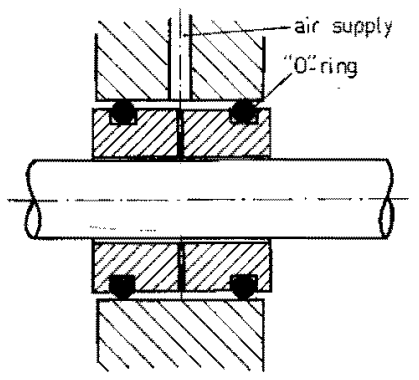

Fig. 4.1. Principle of an aerostatic journal bearing with " $\mathrm{O}$ "-ring support. 
of the foundation tuning, but with very great simplification indeed of the bearings. Mori and Mori ${ }^{4-7,8}$ ) examined the effect of a support by means of an aerostatic sub-bearing in a greatly simplified system.

Relatively little attention has been paid to flexible supports in the case of liquid bearings. Yet the performance of a liquid bearing differs greatly from that of a gas bearing, and the results for gas bearings cannot simply be used for liquid bearings. This different behaviour is caused by, among other things, cavitation in liquid bearings and is due to the fact that, unlike gas bearings, liquid bearings have response coefficients that are frequency-independent.

In this chapter we shall deal with a symmetric, flexibly supported rotor with liquid bearings. In secs $4.2,4.3$ and 4.4 the support is imagined to be built up from springs and dampers. In order to be able to draw a clear distinction between the main effects we shall first consider rotationally symmetric supports. In sec. 4.3 .8 we shall discuss some effects due to rotational asymmetry in the support.

Section 4.5 will be concerned with a so-called floating-bush bearing. The outer bearing in such a system is, as it were, a flexible support of the inner bearing. However, the main difference is that the stiffness of the support depends upon the speed of the rotor, and that the support reaction contains what are known as cross-terms. The stabilising effect of this type of bearing does not seem to have been worked out yet. In an article of Orcutt and $\mathrm{Ng}^{4-4}$ ) the following comment is made on "good" stability.

"There is no clear explanation for the latter attribute (i.e. good stability) since, unlike the tilting-pad or lobe bearings, it has no inherent antiwhirl characteristics."

\subsection{The expected behaviour of a flexible bearing support consisting of springs and dampers}

In chapter 3 it was shown that the proper stability criterion (3.11) could be deduced also by assuming the forces to be in equilibrium at the transition of stability. Analogous considerations will be used in this section to examine the effects to be expected from a flexible bearing support, thus permitting of a convenient interpretation of the results of the more exact calculations in the following sections. In this we shall restrict ourselves to rotationally symmetric systems with negligible gyroscopic effects $(\lambda \approx 0)$ and bearing supports consisting of springs and dampers.

First a remark should be made about the response coefficients. The (nondimensionless) response coefficients of the supports, $A_{s x x}, A_{s y y}, B_{s x x}$, etc., will be constant in a given system. If it is assumed that the (dimensionless) response coefficients of the bearings are functions only of the dimensionless static bearing deflection, $\mathbf{e}(\mathbf{e}=0$ in this section), it follows from the definitions of these coefficients (cf. chapter 2), viz. 
with

$$
a_{b x x}=\frac{\Delta R}{F_{0}} A_{b x x}, \quad b_{b x x}=\frac{\Omega \Delta R}{F_{0}} B_{b x x,}, \quad \text { etc. }
$$

$$
F_{0}=\frac{12 \eta \Omega R^{4}}{(\triangle R)^{2}}
$$

that the damping coefficients, $B_{b x x}$, etc., of a given bearing will be constant but that the stiffness coefficients, $A_{b x x}, A_{b x y}$, etc., will be proportional to the speed of the rotor, $\Omega$. Hence the bearing stiffness will increase with the speed.

In chapter 3 we have seen that, with a rigidly supported bearing which is just on the border of stability, the centre of the shaft can describe a circle about its equilibrium position with an angular velocity $\delta_{c}$. In this situation the (radial) bearing response counterbalances exactly the centrifugal forces acting on the "rotor mass", $1 / \bar{W}$. According to (3.11) this balance of forces is expressed by

$$
\underbrace{\left(a+\delta_{c} b_{t}\right) e}_{\text {radial bearing load }}=\underbrace{\frac{\delta_{c}{ }^{2}}{\bar{W}} e,}_{\text {centrifagal force }}
$$

in which $e$ (cf. fig. $4.2 a$ ) is the dimensionless bearing deflection.

If the bearing is supported not rigidly but elastically, by means of springs with a stiffness $a_{s}$ (fig. $4.2 b$ ), then here again, on the border of stability, the centre of the shaft can describe a circle at an angular velocity $\delta_{c}$. For equilibrium, then, the following should apply:

$$
a_{r} e_{\mathrm{tot}}=\frac{\delta_{c}^{2}}{W} e_{\mathrm{tot}}
$$

in which $a_{r}$ is the total stiffness and $e_{\text {tot }}$ the total deflection (cf. fig. 4.2b). Since $a_{r}<a+\delta_{c} b_{t}$, the "mass", $1 / W$, must be smaller on the border of stability than in the case of fig. $4.2 a$. Hence we can expect the stability of the rotor to decrease with the stiffness of its undamped support.

By adding a (slight) damping to the springs of fig. $4.2 b$ we obtain the situation represented in fig. $4,2 c$. In the support a difference in phase arises between the support deflection $e_{s}$ and the force of reaction. For an "equilibrium situation", then, it is required that the attitude angle $\varphi_{a}$ of the bearing be positive. This is only possible with $\omega_{w}<\delta_{c}$ ( $\omega_{w}$ is the dimensionless whirl velocity), so that the equilibrium must be established at a smaller angular velocity.

In the equation

$$
a_{r} e_{\mathrm{tot}}=\frac{\omega_{w}{ }^{2}}{\bar{W}} e_{\mathrm{tot}}
$$

$a_{r}$ is bigger than in (4.3), due to phase shifts $\alpha$ and $\varphi_{a}$ and, in addition, owing to the fact that as a rule the bearing stiffness will increase with decreasing whirl 


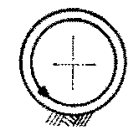

a)
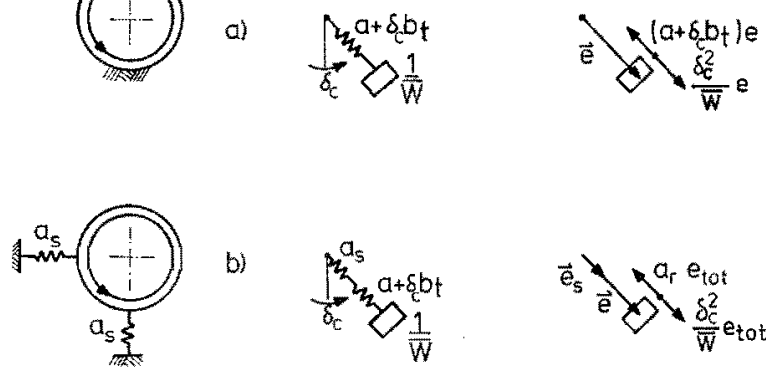

b)
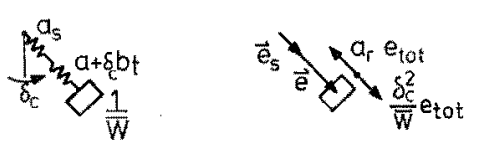

$$
\begin{aligned}
& \frac{1}{a_{r}}=\frac{1}{a_{s}}+\frac{1}{a+s_{c} b_{t}} \\
& \overrightarrow{\mathrm{e}}_{\mathrm{tot}}=\overrightarrow{\mathrm{e}}_{\mathrm{s}}+\overrightarrow{\mathrm{e}}
\end{aligned}
$$

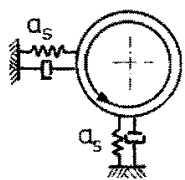

c)

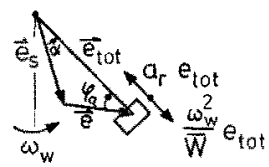

$$
\omega_{w}<\delta_{C}
$$

d)

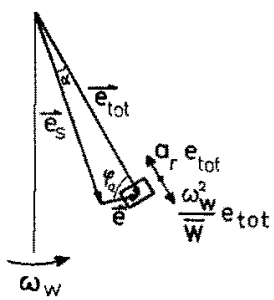

Fig. 4.2. The physical behaviour of a support;

(a) rigid support,

(b) flexible support without damping,

(c) flexible support with slight damping,

(d) flextble support with damping; predominating support deflection.

velocity, $\omega_{w}$. In the situation shown in fig. $4.2 c$ the mass, $1 / \bar{W}$, can be greater than that in the situation of fig. $4.2 b$. Hence we expect that the addition of slight damping to an undamped flexible suspension will improve the stability.

The support illustrated in fig. $4.2 a$ is rigid, so that here the displacement of the bearing predominates over that of the support. Let us now assume that the displacement of the support prevails, owing to the stiffness of the support being low and/or the stiffness of the bearing being high (for example at high $\Omega$ ). We can then conceive of a situation as given in fig. 4.2d. Here $e_{s} \approx e_{\text {tot }}, \varphi_{a}$ has a big value, almost $\pi / 2$, and $e$ will decrease with increasing rotor speed. It is possible to re-establish the equilibrium only if $\alpha$ is smaller, hence at a smaller value of $\omega_{w}$ and consequently at a bigger value of $1 / \bar{W}$. Accordingly we expect that, if the displacement of the bearing decreases in comparison with that of the support, and hence if in the situation of fig. $4.2 c$ the rotor speed is greatly increased, a situation will arise as is shown in fig. $4.2 d$; this is characterised by the fact that the stability is improved by increasing of the rotor speed. 
At very high values of the damping, the support will act as if it were rigid again. We can therefore expect that, at very high values of the damping of the support, we shall find the same values of the stability as with a rigid support.

\subsection{Supports consisting of springs and dampers}

\subsubsection{Equations of motion}

A rotor with flexible bearing supports is shown in fig. 4.3 in which $M_{R}$ is the mass of the rotor, and $M_{s}$ that of the bearing bush; $I$ is the moment of inertia about $\mathrm{bb}^{\prime}$, and $J$ that about an axis in plane aa' passing through $\mathrm{G}$; $Q$ is the angular speed of the rotor, and $L$ is the distance between the centres of the bearings.

We base our considerations on the following assumptions:

- The system and the external load are symmetric with respect to plane aa'.

- The rotor is rigid and rotationally symmetric with respect to $b^{\prime}$.

- The external load is constant.

- The bearing support can be represented by linear springs and dampers.

- The bearing reactions are linear functions, with constant coefficients, of the relative journal displacement, $\mathbf{X}_{b}$, and journal velocity, $(\mathrm{d} / \mathrm{d} t) \mathrm{X}_{b}$.

The coordinates of the centres of the journal and of the bush are given in fig. 4.4. In this figure, $O_{E}$ and $Q_{E}$ are the equilibrium positions of respectively the journal and the bush, while $O$ and $Q$ are the positions of respectively the journal and the bush after a displacement from the equilibrium position. The dynamic deflections of bearing and support are denoted by $\mathbf{X}_{b}$ and $\mathbf{X}_{s}$.

The motion of this system is described by the following dimensionless equations (cf. chapter 2).

Journal forces

$$
\begin{aligned}
& \Delta f_{b x}=-a_{b x x} x_{b}-b_{b x x} \dot{x}_{b}-a_{b x y} y_{b}-b_{b x y} \dot{y}_{b}, \\
& \Delta f_{b y}=-a_{b y y} y_{b}-b_{b y y} \dot{y}_{b}-a_{b y x} x_{b}-b_{b y x} \dot{x}_{b} .
\end{aligned}
$$
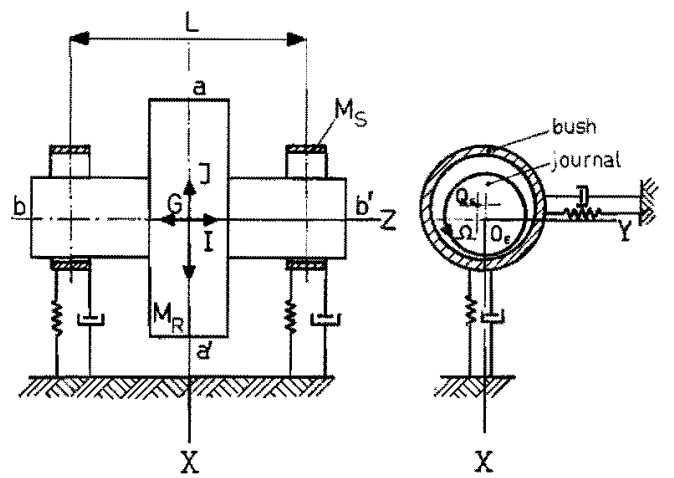

Fig. 4.3. Symmetric rotor-and-bearing system with flexible bearing support. 


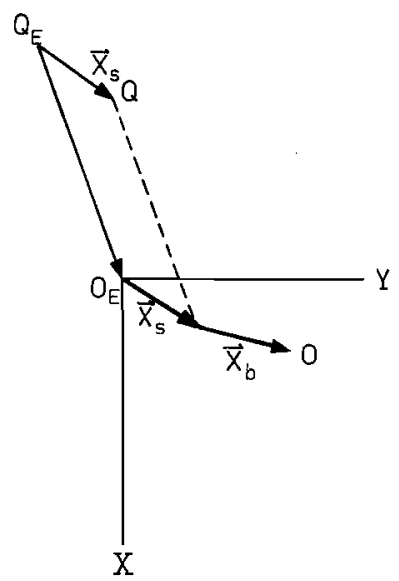

Fig. 4.4. Coordinates of the centres of bush and journal.

$\mathrm{Q}_{\mathrm{E}}=$ static equilibrium position of centre of bush;

$\mathrm{O}_{\mathrm{E}}=$ static equilibrium position of centre of journal;

$\mathrm{Q}=$ deflected position of centre of bush;

$\mathrm{O}=$ deflected position of centre of journal.

Forces on bearing bush

$$
\begin{aligned}
& \Delta f_{s x}=-\Delta f_{b x}-a_{s x x} x_{s}-b_{s x x} \dot{x}_{s}, \\
& \Delta f_{s y}=-\Delta f_{b y}-a_{s y y} y_{s}-b_{s y y} \dot{y}_{s} .
\end{aligned}
$$

Equations of motion of the rotor

$$
\begin{aligned}
& \Delta f_{b x}=\frac{1}{\bar{W}}(\ddot{x}+\lambda \dot{y}), \\
& \Delta f_{b y}=\frac{1}{\bar{W}}(\ddot{y}-\lambda \dot{x}) .
\end{aligned}
$$

Equations of motion of the bearing bush

$$
\begin{aligned}
\Delta f_{s x} & =\frac{m_{s}}{\bar{W}} \ddot{x}_{s}, \\
\Delta f_{s y} & =\frac{m_{s}}{\bar{W}} \ddot{y}_{s} .
\end{aligned}
$$

In the above equations the dimensionless quantities are defined as follows:

$\Delta f_{b x}=\frac{\Delta F_{b x}}{F_{0}}, \quad \Delta f_{b y}=\frac{\Delta F_{b y}}{F_{0}}, \quad \begin{aligned} & \text { dynamic parts of the forces } \\ & \text { acting on the journal; }\end{aligned}$ 
$\Delta f_{s x}=\frac{\Delta F_{s x}}{F_{0}}, \quad \Delta f_{s y}=\frac{\Delta F_{s y}}{F_{0}}, \quad \begin{aligned} & \text { dynamic parts of the forces } \\ & \text { acting on the bush; }\end{aligned}$

$x_{b}=\frac{X_{b}}{\Delta R}, \quad y_{b}=\frac{Y_{b}}{\Delta R}$

deflections of the journal;

$x_{s}=\frac{X_{s}}{\Delta R}, \quad y_{s}=\frac{Y_{s}}{\Delta R}$

deflections of the support;

$a_{b x x}=\frac{\Delta R}{F_{0}} A_{b x x}, \quad$ etc.

stiffness coefficients of the bearing;

$b_{b x x}=\frac{\Omega \Delta R}{F_{0}} B_{b x x}, \quad$ etc.

damping coefficients of the bearing;

$a_{s x x}=\frac{\Delta R}{F_{0}} A_{s x x}, \quad$ etc.

stiffness coefficients of the support;

$b_{s x x}=\frac{\Omega A R}{F_{0}} B_{s x x}, \quad$ etc. $\quad \begin{aligned} & \text { damping coefficients of the } \\ & \text { support; }\end{aligned}$

$W=\left\{\begin{array}{ll}\frac{2 F_{0}}{M_{R} \Delta R \Omega^{2}} & \text { (translational mode) } \\ \frac{F_{0} L^{2}}{2 J \Delta R \Omega^{2}} & \text { (conical mode) }\end{array}\right\}$ stability parameter

$\lambda=\left\{\begin{array}{ll}0 & \text { (translational mode) } \\ \frac{I}{J} & \text { (conical mode) }\end{array}\right\}$ gyroscopic parameter;

$m_{\mathrm{s}}=\left\{\begin{array}{ll}\frac{2 M_{S}}{M_{R}} & \text { (translational mode) } \\ \frac{M_{S} L^{2}}{2 J} & \text { (conical mode) }\end{array}\right\}$ mass of support;

$x=x_{b}+x_{s}, \quad y=y_{b}+y_{s} \quad$ total deflections of the jour nal;

$\tau=\Omega t \quad$ dimensionless time.

The reference force, $F_{0}$ is chosen as follows (cf. chapter 2):

$$
F_{0}=\frac{12 \eta \Omega R^{4}}{(\Delta R)^{2}}
$$




\subsubsection{The choice of the dimensionless support parameters}

It was already mentioned in sec. 4.2 that the bearing response coefficients, $a_{b x x}, a_{b x y}, b_{b x x}, b_{b x y}$, etc., are to be considered solely as functions of the equilibrium position, e. The damping coefficients, $b_{s x x}, b_{s y y}$, are for a given system constants, too (cf. $(4.9 \mathrm{~h})$ and $(4.9 \mathrm{n})$ ), but the stiffness coefficients of the supports become functions of $\Omega$ when made dimensionless (cf. $(4.9 \mathrm{~g})$ and $(4.9 \mathrm{n})$ ).

In representing the stability curves in a diagram it appears to be convenient to have (for a given system and a given e) only one parameter that depends on $\Omega$, and for this reason we shall introduce a new parameter, $k$, that is independent of $\Omega$ :

$$
k=\frac{a_{s x x}}{\bar{W}}=\frac{\Delta R A_{s x x}}{\bar{W} F_{0}}=\frac{1}{288} \frac{M_{R}(\Delta R)^{6}}{\eta^{2}} \frac{R^{8}}{s x x} .
$$

Fuither let

$$
\begin{aligned}
& \xi=\frac{A_{s y y}}{A_{s x x}}, \\
& \xi=\frac{B_{s y y}}{B_{s x x}} \\
& \varrho=b_{s x x} .
\end{aligned}
$$

We can then rewrite (4.6) as

$$
\begin{aligned}
& \Delta f_{s x}=-\Delta f_{b x}-\bar{W} k x_{s}-\varrho \dot{x}_{s}, \\
& \Delta f_{s y}=-\Delta f_{b y}-\zeta \bar{W} k y_{s}-\xi \varrho \dot{y}_{s} .
\end{aligned}
$$

Now (4.11), (4.5), (4.7) and (4.8) describe the motion of the system, and for a given system and a prescribed equilibrium position, $\mathbf{e}\left(=\mathbf{Q}_{\mathrm{E}} \mathbf{O}_{\mathrm{E}}\right)$, the stability is governed solely by the stability parameter, $\bar{W}$. The latter can be interpreted as the reciprocal value of the dimensionless angular speed of the rotor, since $\bar{W}$ is the only parameter still containing $\Omega$. For translation it follows from (4.9i) and (4.9n) that

$$
W=\frac{24 \eta R^{4}}{M_{R}(\Delta R)^{3}} \frac{1}{\Omega},
$$

and for the conical movement:

$$
\bar{W}=\frac{6 \eta R^{4} L^{2}}{J(\Delta R)^{3}} \frac{1}{\Omega}
$$

4.3.3. The characteristic equation if the mass of the support and the gyroscopic effects are negligible

In many cases the mass of the support is small as compared with the mass 
(or the reduced moment of inertia) of the rotor $\left(m_{s} \ll 1\right)$ and the influence of gyroscopic effects is only slight $(\lambda \approx 0)$.

Hence in what follows we shall assume the effect of the mass of the support and the gyroscopic effects to be negligible and put

$$
m_{s}=\lambda=0 \text {. }
$$

It may be noted here that from chapter 2 it appears that gyroscopic effects usually have a stabilising influence, so that, as far as stability is concerned, one errs on the safe side in neglecting them. From (4.8) we derive

$$
\Delta f_{s x}=\Delta f_{s y}=0 .
$$

With the usual substitutions $\left(x_{b}=\hat{x}_{b} \exp (s \tau), y_{b}=\hat{y}_{b} \exp (s \tau)\right.$, etc.) this leads to the characteristic equation of the system given by (4.11), (4.5), (4.7) and (4.8):

$$
\left|\begin{array}{cccc}
s^{2}+b_{11} s+a_{11} & b_{12} s+a_{12} & s^{2} & 0 \\
b_{21} s+a_{21} & s^{2}+b_{22} s+a_{22} & 0 & s^{2} \\
s^{2} & 0 & s^{2}+b_{33} s+a_{33} & b_{34} s+a_{34} \\
0 & s^{2} & b_{43} s+a_{43} & s^{2}+b_{44} s+a_{44}
\end{array}\right|=0
$$

or

$$
a_{0} s^{6}+a_{1} s^{5}+a_{2} s^{4}+a_{3} s^{3}+a_{4} s^{2}+a_{5} s+a_{6}=0,
$$

in which

$$
\begin{array}{ll}
a_{11}=\bar{W} a_{b x x}, & b_{11}=\bar{W} b_{b x x}, \\
a_{12}=\bar{W} a_{b x y}, & b_{12}=\bar{W} b_{b x y}, \\
a_{21}=\bar{W} a_{b y x}, & b_{21}=\bar{W} b_{b y x}, \\
a_{22}=\bar{W} a_{b y y}, & b_{22}=\bar{W} b_{b y y}, \\
a_{33}=\bar{W}^{2} k, & b_{33}=\bar{W} \varrho, \\
a_{34}=0, & b_{34}=0, \\
a_{43}=0, & b_{43}=0, \\
a_{44}=\zeta \bar{W}^{2} k, & b_{44}=\xi \bar{W} \varrho .
\end{array}
$$

The coefficients $a_{0}$ to $a_{6}$ of the polynomial are given in appendix II.

Using the Routh-Hurwitz criterion we can determine from the coefficients $a_{0}$ to $a_{6}$ whether the motion is stable or unstable for a given value of the parameter $\bar{W}$ : on the border of stability and instability one of the roots of (4.14b) is purely imaginary. Utilising this property, this root can be evaluated from (4.14b), and $\hat{x}_{b}, \hat{y}_{b}$, etc., can be calculated from (4.14a). 
4.3.4. Application to an $A L G$ bearing and a support having rotationally symmetric response

The ALG bearing (axial lubricant groove bearing) is described in more detail in chapter 7; the lubricant is supplied to this bearing through an axial groove at the location of the widest film gap; in this groove, at the two bearing ends and in the cavitation region the pressure is assumed to be zero.

In most cases small variations in shape and location of the lubricant grooves have only slight effects on the load-carrying capacity and the bearing coefficients, so that the ALG bearing may be considered as representative of many smooth cylindrical bearings.

Figures $4.5 a$ to $4.5 d$ show the stability curves of the system of fig. 4.3 in which the support has rotational symmetry $(\zeta=\xi=1)$. Here the stability number, $\bar{W}$, and the support damping, $\varrho$, determine the positions of the stable and unstable regions. The static bearing eccentricities, $e_{x}$ and $e_{y}$, and the support stiffness, $k$, are also used as parameters.

From the foregoing it follows that, for translation,

$$
\begin{gathered}
\bar{W}=\frac{2 F_{0}}{M_{R} \Omega^{2} \Delta R}=\frac{24 \eta R^{4}}{M_{R}(\Delta R)^{3}} \frac{1}{\Omega} \\
\varrho=\frac{\Delta R \Omega}{F_{0}} B_{s x x}=\frac{(\Delta R)^{3}}{12 \eta R^{4}} B_{s x x} \\
k=\frac{\Delta R}{\bar{W}} \frac{A_{s x x}}{F_{0}}=\frac{M_{R}(\Delta R)^{6}}{288 \eta^{2} R^{8}} A_{s x x}, \\
\zeta=\xi=1, \\
\lambda=0
\end{gathered}
$$

and for conical motion:

$$
\begin{aligned}
\bar{W}=\frac{F_{0} L^{2}}{2 J \Delta R \Omega^{2}} & =\frac{6 \eta R^{4} L^{2}}{J(\Delta R)^{3}} \frac{1}{\Omega}, \\
\varrho=\frac{\Delta R \Omega}{F_{0}} B_{s x x} & =\frac{(\Delta R)^{3}}{12 \eta R^{4}} B_{s x x}, \\
k=\frac{\Delta R}{\bar{W}} \frac{A_{s x x}}{F_{0}} & =\frac{J(\Delta R)^{3}}{72 \eta^{2} R^{8} L^{2}} A_{s x x}, \\
\zeta & =\xi=1, \\
\lambda & =0 .
\end{aligned}
$$


In fig. $4.6 a$ we may regard the stable and unstable regions, bounded by the curves $\mathrm{A}$ and $\mathrm{B}$, as the fundamental form, from which all the other figures can be derived. This fundamental form can also be seen in fig. $4.5 c$ at $k=0 \cdot 1$.

On the left there is, with slight damping, an unstable region bounded by curve A. At point 1 the stability is less than with a rigid support, due to the destabilizing effect caused by the flexibility of the support at low damping. As we pass from 1 to 2, the stability is improved as the damping increases. At point 3 the bearing is much stiffer than at 1, due to the higher speed (cf. sec. 4.2). Here the stability is enhanced by an increase in the speed (decreasing of $\bar{W}$ ), and again the unstable region is reduced owing to the increase of the damping. If the damping exceeds that at point 4 , then the rotor is found to become stable for all values of $\bar{W}$ and hence for all speeds.

At high damping the support becomes rigid again, and consequently the value of $\bar{W}$ at point 5 corresponds to that of $\bar{W}$ for a rigid support.

A decrease in the stiffness of the support, $k$, causes curve A to shift upwards and to the left $\left(A_{1}\right)$. The inherently stable region is then widened. An increase in the stiffness of the support narrows the inherently stable region $\left(A_{2}\right)$. This may even give rise to a degeneration, as shown in fig. $4.6 b$ (and fig. $4.5 c$ with $k=1$ ). Curves $\mathrm{A}$ and $\mathrm{B}$ will then coalesce, a small stable region being left below the unstable region. With a construction having the line of action $p q$ it is possible, by a rise in the speed, to start at $p$, then pass through the unstable region and arrive again at the stable point $\mathrm{q}$.

If the bearing itself has better stability properties because it operates at a bigger static displacement, $e_{x}$, then this also manifests itself in the stability curves: at equal values of $k$ and $\varrho$ the unstable regions appear to decrease with increasing $e_{x}$ (cf. fig. 4.5).

It is seen from fig. 7.7 that a rigidly supported ALG bearing is inherently stable if $e_{x}>$ ca 0.8 ; if the support is flexible, the stability turns out to be satisfactory also: no instability was found in the region $e_{x}>$ ca 0.8 .

At $e_{x}=0$ a rigidly supported ALG bearing is inherently unstable (cf. fig. 7.7), but with a flexible support a stable region, bounded by the $\varrho$-axis, appears still to exist. Accordingly, a flexibly supported rotor with ALG bearings may, after having been unstable at low speeds, become stable again by a sufficient increase in the speed (cf. fig. 4.5a).

\subsubsection{Application to an ORS bearing and a support having rotationally sym- metric response}

By an ORS bearing (cf. chapter 7) we understand a helical-groove journal bearing with a groove pattern such that the radial stiffness is optimum at $e=|\mathbf{e}|=0$. This bearing, in contrast to an ALG bearing, possesses adequate stability properties even at $|\mathbf{e}|=0$, and with rigid supports (cf. fig. 7.18). The results with a flexible support are given in fig. 4.7. At $e_{x}=0$ (fig. 4.7a) 

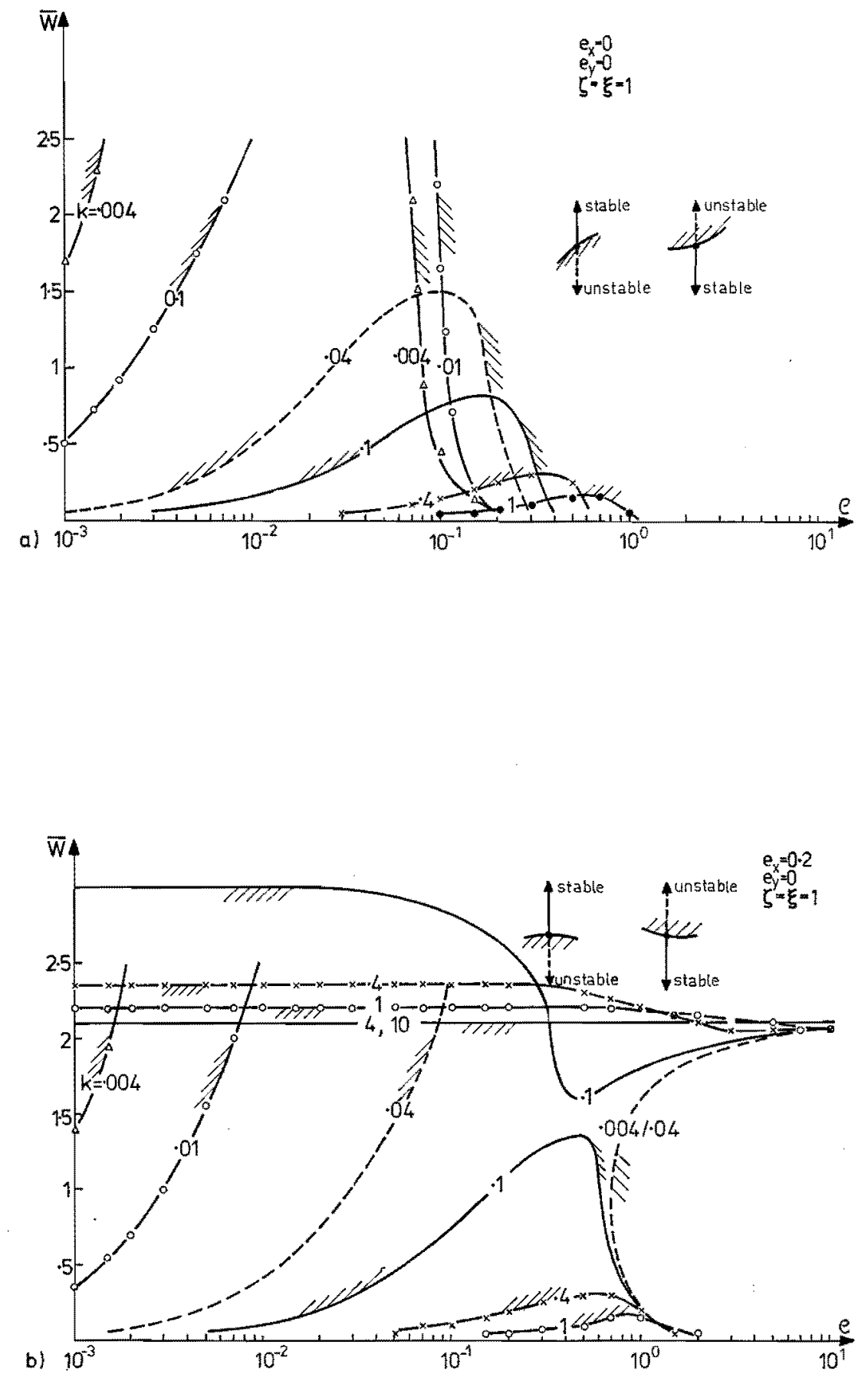

Fig. 4.5. Stability curves of a rotor with $A L G$ bearings $(B / D=1)$ and fexible bearing supports. $(a): e_{x}=0 ;(b): e_{x}=0.2 ;(c) ; e_{x}=0.4 ;(d): e_{x}=0.6$. 

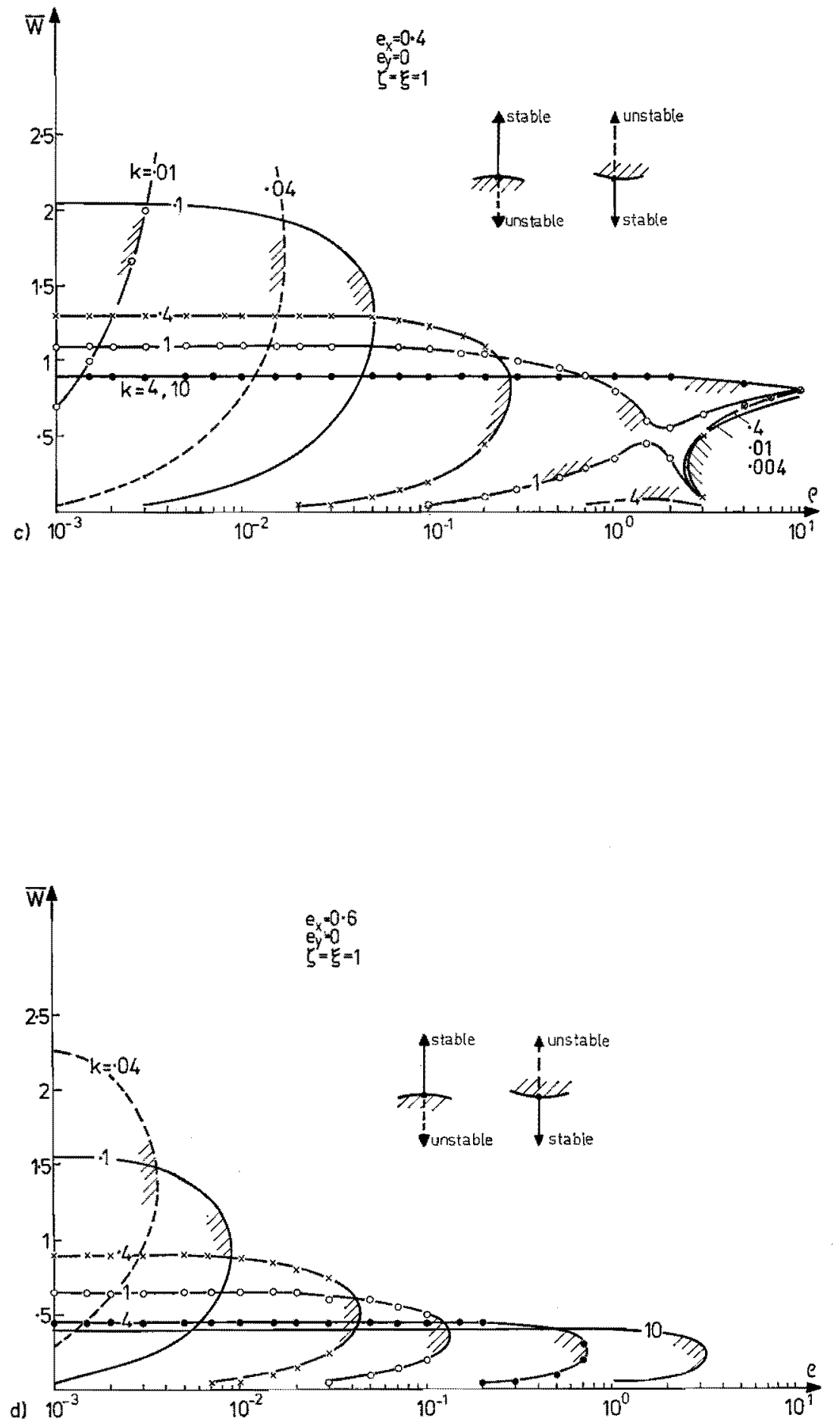

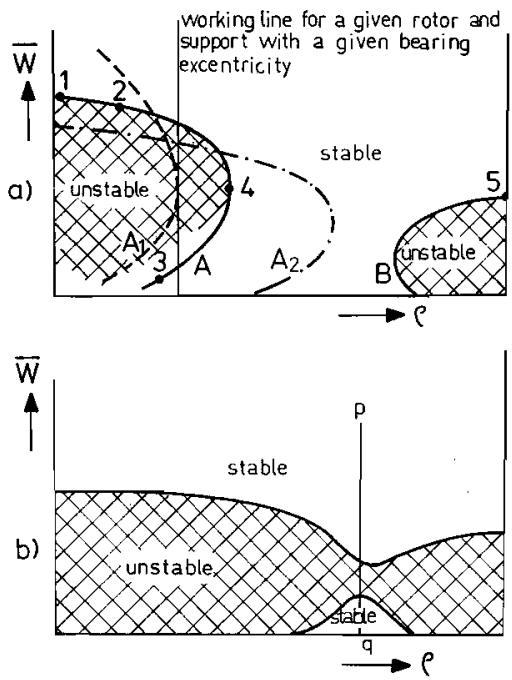

Fig. 4.6. Typical shape of stability curves for a rotor with flexible supports. (a) Lobe-shaped instability regions; $(b)$ degeneration of $(a)$.

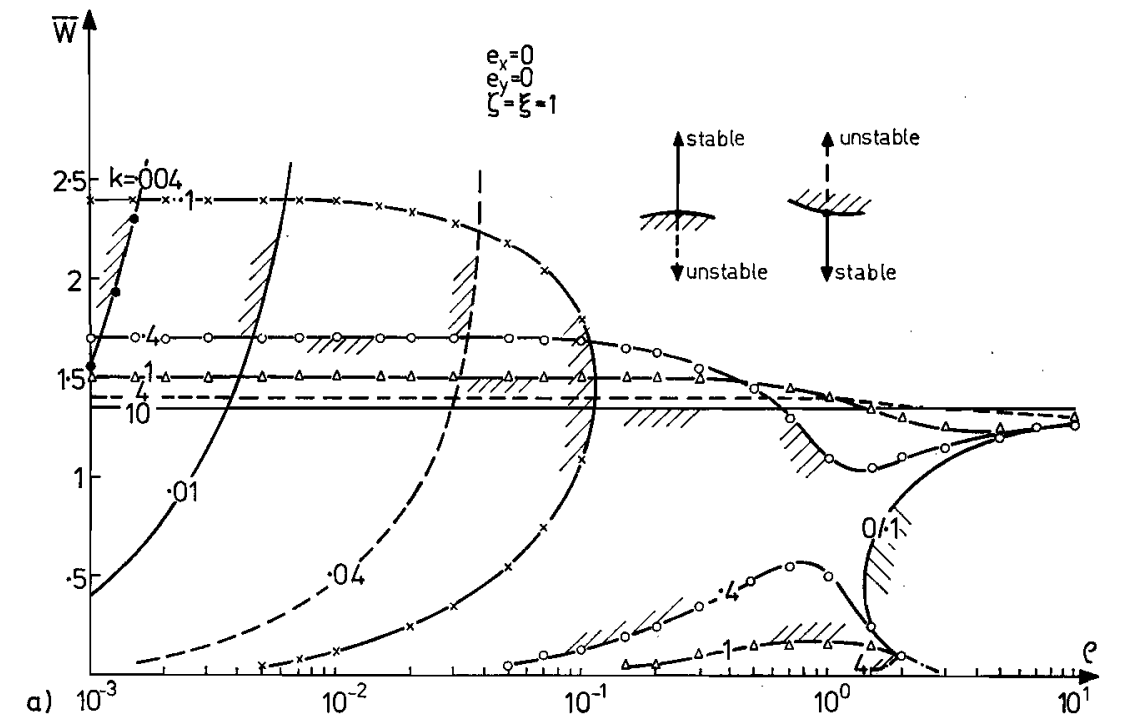

Fig. 4.7. Stability curves of a rotor with ORS bearings $(B / D=1)$ and flexible bearing supports. $(a): e_{x}=0 ;(b): e_{x}=0.4 ;(c): e_{x}=0.8$. 

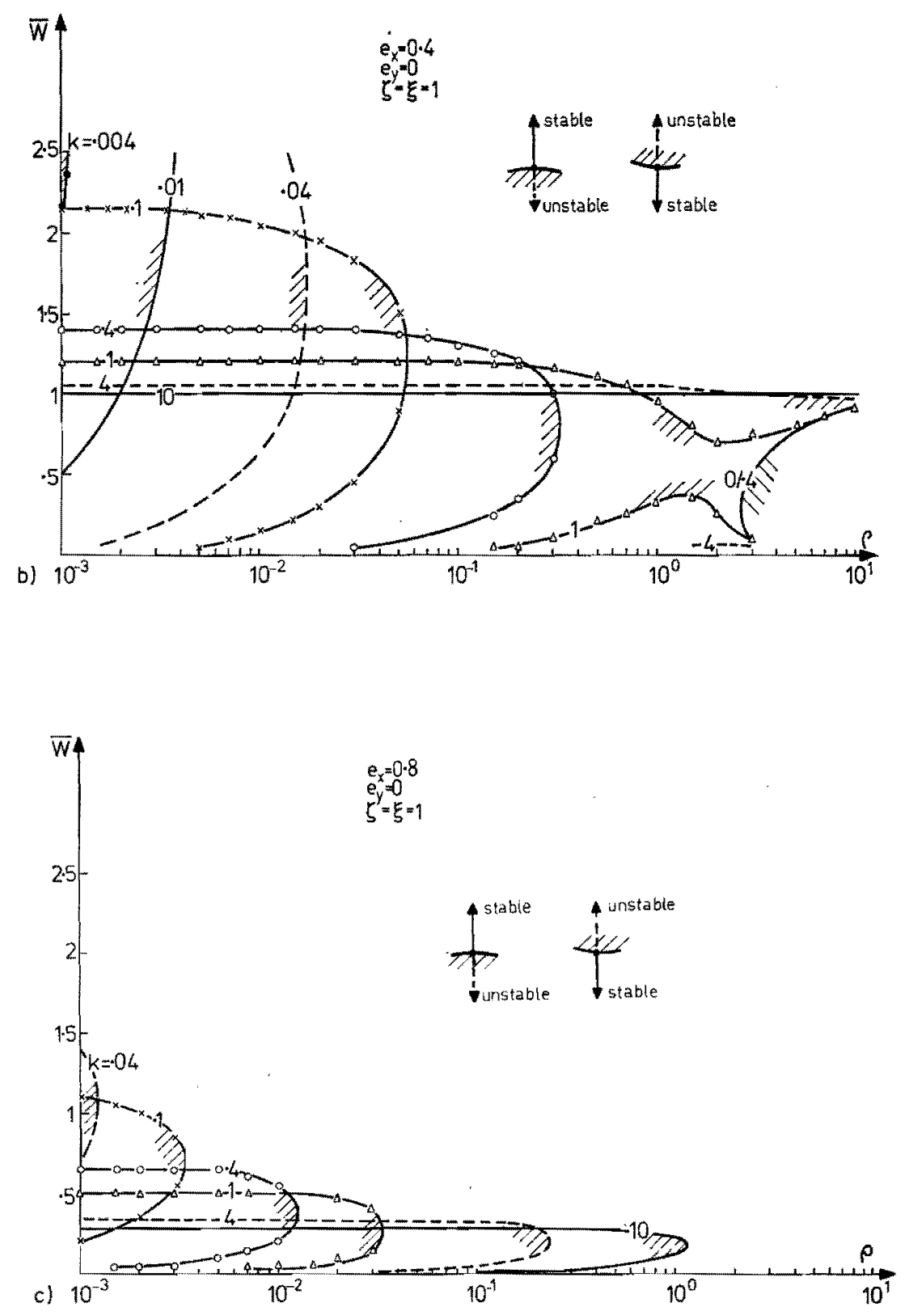

the stability is much better than that of an ALG bearing under the same circumstances (fig. 4.5a). Because the response coefficients of an ORS bearing do not vary so greatly with $e_{x}$, the increase in stability with increasing $e_{x}$ is much smaller than with an ALG bearing. 
While fig. 4.7 gives the stability curves for an ORS bearing with $B / D=1$, in fig. 4.8 they are given for $B / D=0 \cdot 5$. The stability with $B / D=0.5$ is distinctly inferior to that of the bearing with $B / D=1$. This is a logical consequence of the smaller response coefficients.
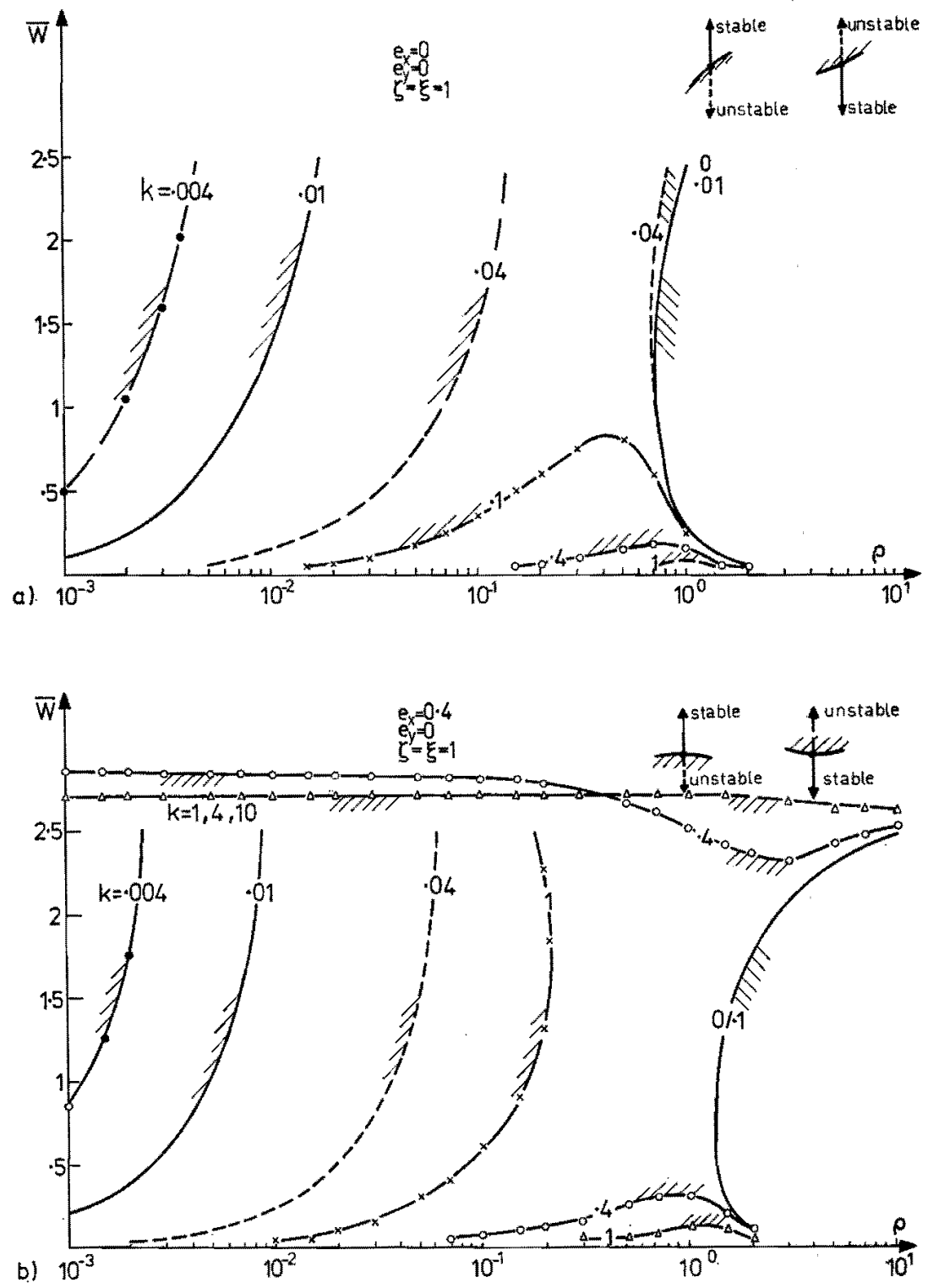

Fig. 4.8. Stability curves of a rotor with ORS bearings $(B / D=0.5)$ and flexible bearing supports. $(a): e_{x}=0 ;(b): e_{x}=0.4 ;(c): e_{x}=0.8$. 


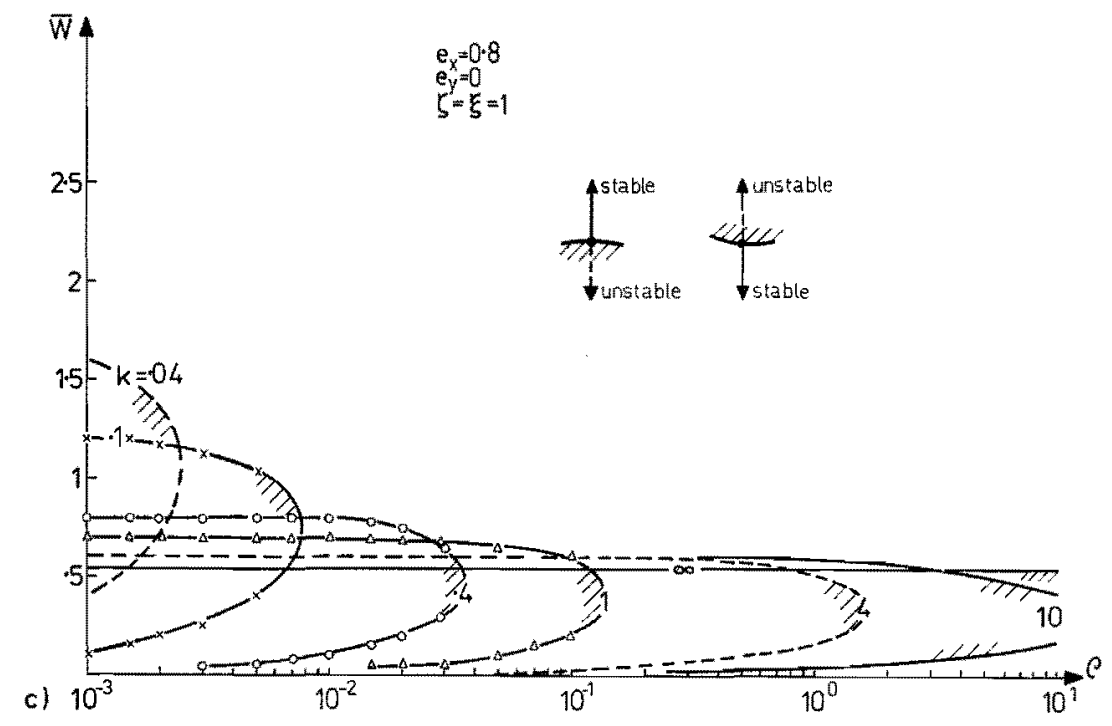

\section{3,6. Example of the application of the stability diagrants}

The manner in which the stability curves can be used is explained by means of the following example.

Problem. It is required to ascertain whether a symmetric rotor-and-bearing system with a vertical rotor (unloaded journal bearings, cf. fig. 4.9) and with rigidly supported ORS bearings is stable, and if not, what flexible support will ensure stability.

The data of the system are:

mass of the rotor

moment of inertia of the rotor

$: M_{R}=10 \mathrm{~kg}$

bearing distance

: $J=0.033 \mathrm{~kg} \mathrm{~m}^{2}$,

angular velocity

: $L=0.20 \mathrm{~m}$,

radius of bearings

$: \Omega=2000 \mathrm{rad} / \mathrm{s}$,

$: R=6: 10^{-3} \mathrm{~m}$,

length of bearings

: $B=12 \cdot 10^{-3} \mathrm{~m}$,

radial clearance

$: A R=20 \cdot 10^{-6} \mathrm{~m}$,

viscosity of lubricant

$: \eta=5 \cdot 10^{-3} \mathrm{Ns} / \mathrm{m}^{2}$.

Furthermore let $\lambda \approx 0$; then $J / L^{2}=0.83, J / L^{2} \ll M_{R}$, and it follows from the definition of $W$ that, with rigid supports, only the translational mode will assume importance. According to (4.9n),

$$
F_{0}=\frac{12 \eta \Omega R^{4}}{(\Delta R)^{2}}=390 \text { newtons, }
$$




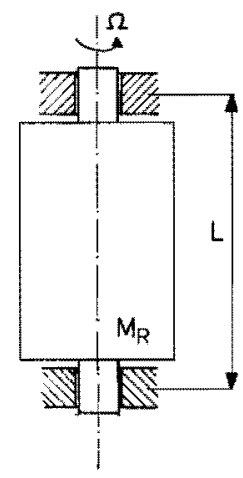

Fig. 4.9. Symmetric vertical rotor.

and, with (4.9i),

$$
\bar{W}(\text { translational mode })=\frac{2 F_{0}}{M_{R} Q^{2} \Delta R}=0.98 .
$$

From fig. 7.18 (or fig. $4.7 a$, extrapolating to $k=\infty$ ) it follows that the rotor is unstable for $\bar{W}<$ ca $1 \cdot 3$ !

Is it possible to stabilise the rotor by means of rotationally symmetric, flexible bearing supports if, on account of design considerations, the minimum support stiffness is allowed to be $2.10^{5} \mathrm{~N} / \mathrm{m}$ ? Because a low stiffness favours the stability in this case we choose the given minimum value and obtain:

$$
k=\frac{\Delta R}{W} \frac{A_{s x x}}{F_{0}}=\frac{20.10^{-6}}{0.98} \times \frac{2.10^{5}}{390} \approx 0.01 .
$$

Figure $4.7 a$ then shows that, for a stable translational mode, the support damping must lie in the region of

$$
\text { ca } 3 \cdot 10^{-3}<\varrho<\text { ca } 2 .
$$

For the conical mode, however, $\bar{W}$ (conical mode) $=\left(M_{R} L^{2} / J\right) \times 0.98=11 \cdot 8$. To prevent instability in this mode we can narrow the limits of $\varrho$, so that the

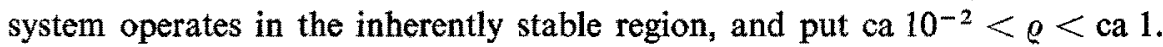

\subsubsection{Possibilities of designing a flexible support}

In principle a flexible support can easily be realised with the aid of an O-ring (fig. 4.10a). This construction has the great drawback, however, that the support damping depends on the stiffness, and vice versa: at a given stiffness the damping is often insufficient to ensure stability (especially with liquid-lubricated bearings). This disadvantage is absent in the construction of fig. $4.10 b$, because the stiffness is brought about by the two O-rings and the damping is predominantly effected by the squeeze bearing, which is capable of operating with 

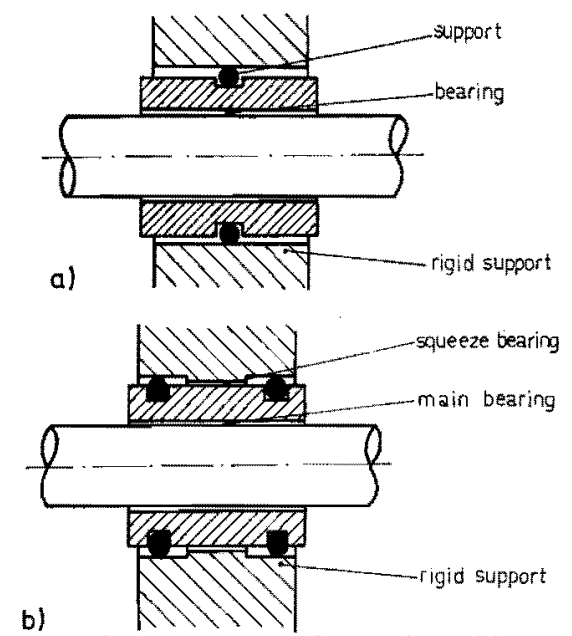

Fig, 4.10. (a) Bearing with "O"-ring support. (b) Bearing with "O"-ring support and additional squeeze bearing.

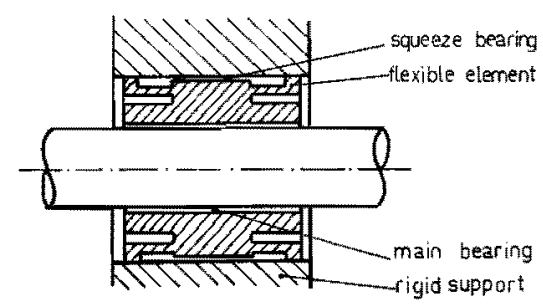

Fig. 4.11. Bearing with flexible support.

the same lubricant as that of the main bearing. In the design of such a squeeze bearing it is possible to select the damping within wide limits.

If the stiffness that can be reached with an O-ring support is insufficient, then constructions of the type shown in fig. $4.11 \mathrm{can}$ be used. Here the collar of the bearing bush represents the spring element. Damping is again accomplished by the squeeze bearing.

\subsubsection{Asymmetric supports}

It will be clear that it is not always possible to rely on rotational symmetry of a support. Supports without rotational symmetry also occur, and sometimes they are certainly designed intentionally in that form. Restricting ourselves to a support as shown in fig. 4.3 we can express the asymmetry in the support stiffness by the parameter $\zeta$ (eq. (4.10b)) and that in the support damping by $\xi$ (eq. $(4,10 \mathrm{c})$ ). Now the number of parameters determining the stability of a rotor-and-bearing system becomes so great as to make it impossible to describe all the cases occurring in practice. Hence we shall confine ourselves to just one example, in which the effect of the introduction of rotational asymmetry in an originally symmetric support will be considered. 
Figure $4.12 a$ shows for an ORS bearing $(B / D=1)$ with a symmetric support $(\xi=\xi=1)$, the effect of a reduction of the stiffness in the $Y$-direction and therefore a reduction in $\zeta$.

It will be seen that reduction of $\zeta$ has a slight effect on the right-hand curve and that initially the left-hand curve shifts to the left, whereby the inherently stable region is widened. At $\zeta \approx 5$ there arises a second lobe, which impedes further extension of the inherently stable region.
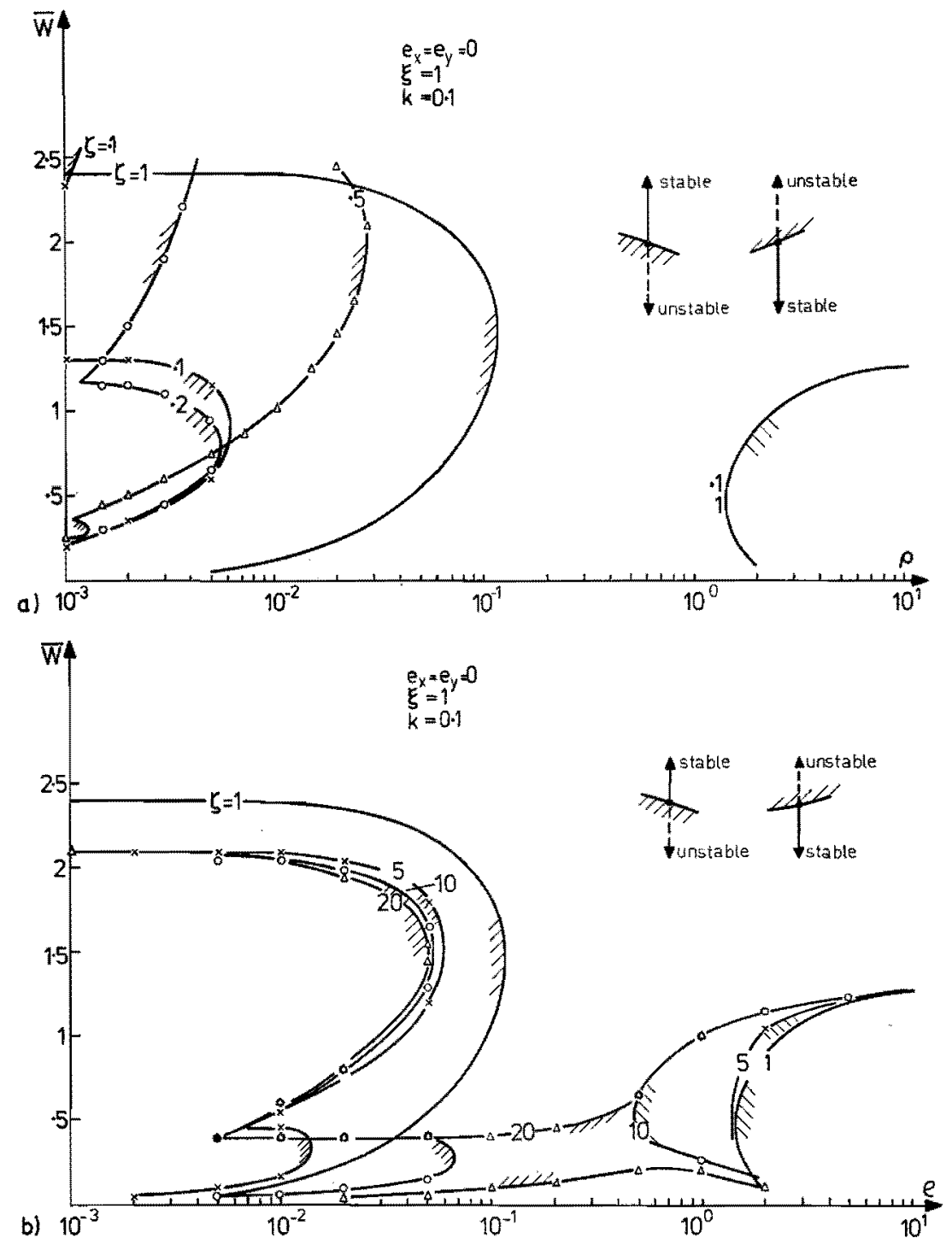

Fig. 4.12. Stability curves of a rotor with ORS bearings $(B / D=1)$ with symmetric support damping and asymmetric stiffness, $e_{x}=0$. 
Figure $4.12 b$ illustrates the effect of an increase in $\zeta$. Here again the lefthand curve divides into two lobes, but an increase in $\zeta$ ultimately causes the inherently stable region to vanish.

It is striking that both a slight increase and a slight decrease of $\zeta$ should enlarge the stable region. This effect is likely to be due to the fact that the motions in the $X$-and $Y$-directions are uncoupled, impeding the transfer of energy and thus producing the same effect as that caused by the enhancement of the damping in a system with rotational symmetry.

The fact that new lobes come into being shows that, for a certain asymmetry, other forms of motion will start to play a role and govern the stability of the system. Inspection of fig. 4.13, which shows the effect of asymmetry in damping on an ORS bearing, reveals that the fundamental form of the diagram remains the same and that the increasing or decreasing of $\xi$ is of the nature of an increase or decrease in the mean damping.

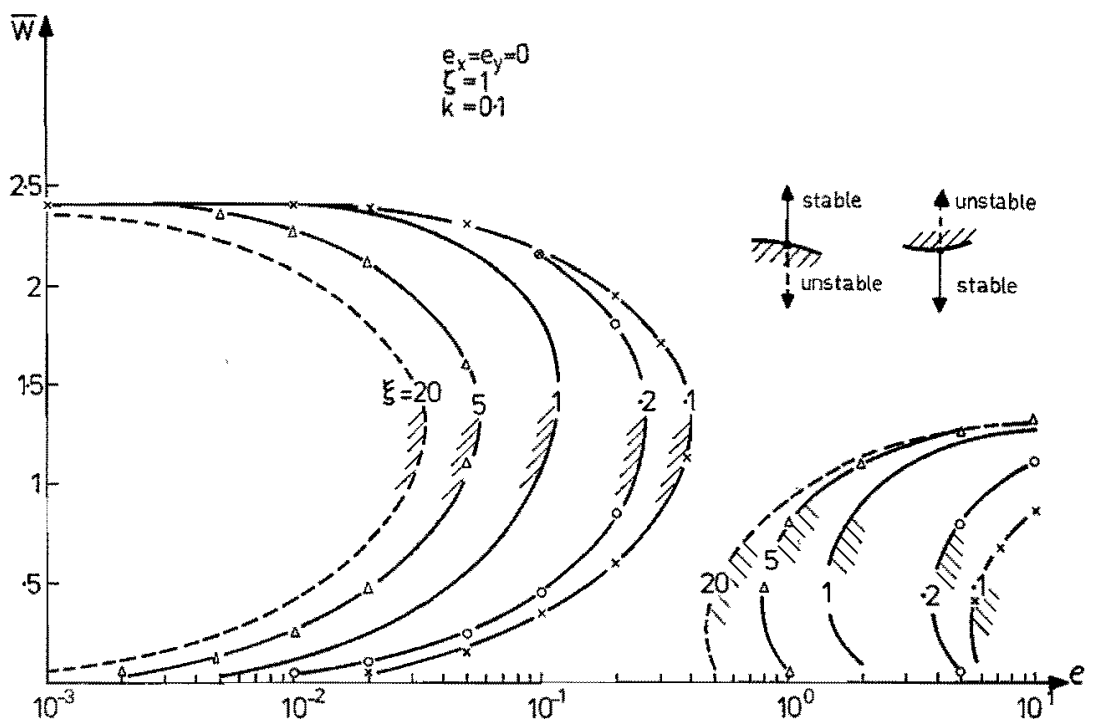

Fig. 4.13. Stability curves of a rotor with ORS bearings $(B / D=1)$ with symmetric support stiffness and asymmetric support damping, $e_{x}=0$.

\subsection{Simplified method of calculation applicable if the bearings as well as the supports have rotational symmetry}

\subsubsection{Analysis}

If both the bearings and the supports have rotational symmetry, then, at the border of stability, the centre of the journal can describe a circle about the equilibrium position. In that situation the responses of the bearing and of the support just balance the centrifugal forces acting on the rotor (cf. chapter 3 
and sec. 4.2). With the help of the equilibrium of forces the calculation of points on the borderline of stability becomes a fairly simple matter.

In what follows we shall again take $m_{s}=\lambda=0$. To the bearing response of a bearing with rotational symmetry the following relations apply (cf. (3.3)):

$$
\begin{aligned}
& \Delta f_{b x}=-a x_{b}-b \dot{x}_{b}-\delta_{c} b y_{b}-b_{t} \dot{y}_{b}, \\
& \Delta f_{b y}=-a y_{b}-b \dot{y}_{b}+\delta_{c} b x_{b}+b_{t} \dot{x}_{b},
\end{aligned}
$$

or in complex form

$$
\Delta f_{b}=\left(-a+\mathrm{j} \delta_{c} b\right) z_{b}+\left(-b+\mathrm{j} b_{t}\right) \dot{z}_{b},
$$

with $z_{b}$ the complex bearing deflection and $\Delta f_{b}$ the complex dynamic force.

Writing $z_{s}$ for the complex support deflection and $z$ for the total journal deflection, we may introduce complex amplitudes $\hat{z}_{b}, \hat{z}_{s}$, and $\hat{z}$, as follows (cf. fig. 4.14):

$$
z_{b}=\hat{z}_{b} \exp \left(\mathrm{j} \omega_{w} \tau\right), z_{s}=\hat{z}_{s} \exp \left(\mathrm{j} \omega_{w} \tau\right), z=z_{b}+z_{s}=\hat{z} \exp \left(\mathrm{j} \omega_{w} \tau\right),
$$
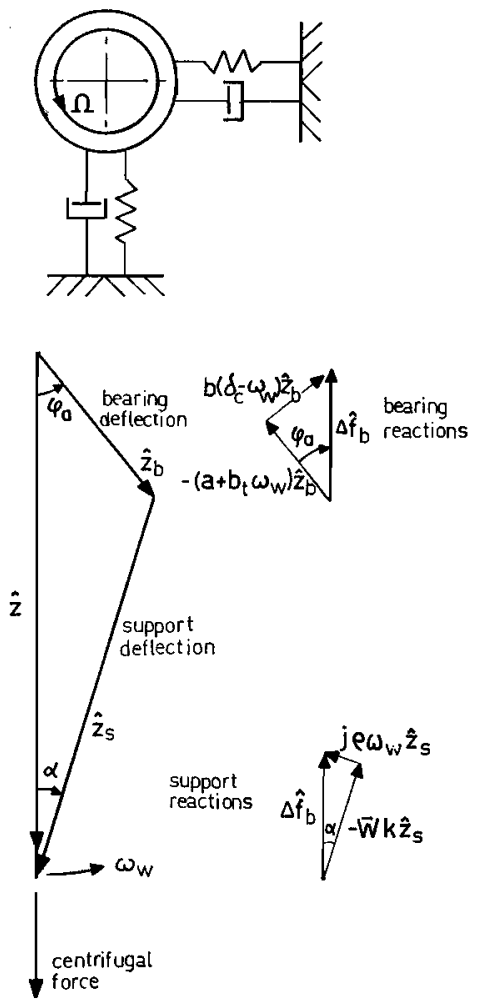

Fig. 4.14. Forces and deflections at the boundary of stability. 
in which $\omega_{w}$ is the dimensionless angular velocity. Using (4.19) and $4 f_{b}=$ $\widehat{\Delta f_{b}} \exp \left(\mathrm{j} \omega_{w} \tau\right)$ we obtain from (4.18b):

$$
\widehat{\Delta f_{b}}=\left[-a-b_{t} \omega_{w}+\mathrm{j} b\left(\delta_{c}-\omega_{w}\right)\right] \hat{z}_{b},
$$

and hence we find

$$
\begin{aligned}
& \left|\hat{\Delta f_{b}}\right|=\left|\hat{z}_{b}\right|\left[\left(a+b_{t} \omega_{w}\right)^{2}+b^{2}\left(\delta_{c}-\omega_{w}\right)^{2}\right]^{1 / 2}, \\
& \cos \varphi_{a}=\frac{\left|\left(a+b_{t} \omega_{w}\right) \hat{z}_{b}\right|}{\left|\hat{A} f_{b}\right|}, \quad \sin \varphi_{a}=\frac{\left|b\left(\delta_{c}-\omega_{w}\right) \hat{z}_{b}\right|}{\left|\hat{U} f_{b}\right|} .
\end{aligned}
$$

Likewise for the support (assuming $\bar{W}>0, k>0, \varrho>0, \omega_{w}>0$ ) we find:

$$
\begin{gathered}
\hat{\Delta f_{b}}=\left(-\bar{W} k-\mathrm{j} \varrho \omega_{w}\right) \hat{z}_{s}, \\
\left|\hat{\Delta f_{b}}\right|=\left|\hat{z}_{s}\right|\left[(W k)^{2}+\left(\varrho \omega_{w}\right)^{2}\right]^{1 / 2}, \\
\tan \alpha=\frac{\varrho \omega_{w}}{\mathscr{W} k},
\end{gathered}
$$

and from fig. 4.14:

$$
\sin \alpha=\frac{\left|\hat{z}_{b}\right| \sin \varphi_{a}}{\left|\hat{z}_{s}\right|} .
$$

It follows from fig. 4.14 that

$$
W=\frac{\left|\hat{\Delta f}_{b}\right|}{\left|\hat{z}_{s}\right|} \frac{\cos \alpha}{k}
$$

and from (4.25) and (4.26)

$$
\varrho=\frac{\bar{W} k}{\omega_{w}} \frac{\left|\hat{z_{b}}\right| \sin \varphi_{a}}{\left|\hat{z_{s}}\right|\left[1-\left(\left|\hat{z_{b}}\right| \sin \varphi_{a} /\left|\hat{z}_{s}\right|\right)^{2}\right]^{1 / 2}} .
$$

With $\lambda=0$ we obtain from (4.7):

$$
\begin{aligned}
& \Delta f_{b}=\frac{1}{\bar{W}} \ddot{z}, \\
& \Delta f_{b}=\frac{\omega_{w}^{2}}{\bar{W}}\left(\left|\hat{z_{b}}\right| \cos \varphi_{a}+\left|\hat{z_{s}}\right| \cos \alpha\right),
\end{aligned}
$$

and from (4.27) and (4.30):

$$
\left|\hat{z}_{s}\right|=-\frac{\left|\hat{z}_{b}\right| \cos \varphi_{a}}{2 \cos \alpha}+\left[\left(\frac{\left|\hat{z}_{b}\right| \cos \varphi_{a}}{2 \cos \alpha}\right)^{2}+\frac{\left|\hat{4 f}_{b}\right|^{2}}{k \omega_{w}{ }^{2}}\right]^{1 / 2} .
$$


Now the calculation of a point of the borderline of stability may proceed as follows:

- Take $\left|\hat{z}_{b}\right|=1$.

- Select $k$ and $\omega_{w}\left(0<\omega_{w}<\delta_{c}\right)$.

- From (4.21) find $\left|\hat{\Delta f_{b}}\right|$.

- From (4.22) determine $\cos \varphi_{a}$ and $\sin \varphi_{a}$.

- Estimate $\cos \alpha$ (usually $\cos \alpha \approx 1$, provided that the damping is not too strong).

- From (4.31) find $\left|\hat{z_{s}}\right|$.

- From (4.27) calculate $\bar{W}$.

- From (4.28) calculate $\varrho$.

- By means of (4.26) check whether the estimate of $\cos \alpha$ was sufficiently accurate, and try again if this is not the case.

\subsubsection{Examples}

In the following examples a number of borderline points of fig. $4.7 a$ will be calculated with the above, simplified theory. In fig. $4.7 a$ an ORS bearing with $B / D=1$ has been considered. From fig. 7.16 its follows that for that bearing, and with $e_{x}=0: a \approx 0.2, b \approx 0.6, \delta_{c} \approx 0.5$ and $b_{t} \approx 0$.

Example 1

Let us choose $\omega_{w}=0.05, k=0 \cdot 1$.

Then from (4.21): $\left|\hat{\Delta f_{b}}\right|=0.338$, from (4.22): $\cos \varphi_{a}=0.59, \sin \varphi_{a}=0.80$.

Take $\cos \alpha \approx 1$.

Then from (4.31): $\left|\hat{z}_{s}\right|=21 \cdot 4$, from (4.27): $W=0 \cdot 16$, from (4.28): $\varrho=1 \cdot 2 \cdot 10^{-2}$, from (4.26): $\sin \alpha=0.037 \Rightarrow \cos \alpha \approx 1$.

The values of $\bar{W}$ and $\varrho$ thus found appear to correspond to those of fig. $4.7 a$.

Example 2

Let us choose $\omega_{w}=0 \cdot 2, k=0 \cdot 1$.

Analogously we find $\bar{W}=0.69$ and $Q=5 \cdot 4 \cdot 10^{-2}$.

Example 3

Let us choose $\omega_{w}=0.45, k=0.1$.

Analogously we find $W=2.04$ and $\varrho=6 \cdot 7 \cdot 10^{-2}$.

Note. The first example clearly shows the low angular velocity $\left(\omega_{w}=0.1 \delta_{c}\right)$ at which the journal centre orbits at the lower end of the borderline of stability. 


\subsection{Bearing supported by an additional bearing (floating-bush bearing)}

\subsubsection{Equations of motion and characteristic equation}

The floating-bush bearing is a more general form of a flexibly supported bearing, in the sense that the force of reaction of the support is governed here by eight response coefficients instead of the four found in the system of fig. 4.3.

A rotor with floating-bush bearings is schematically represented in fig. 4.15.

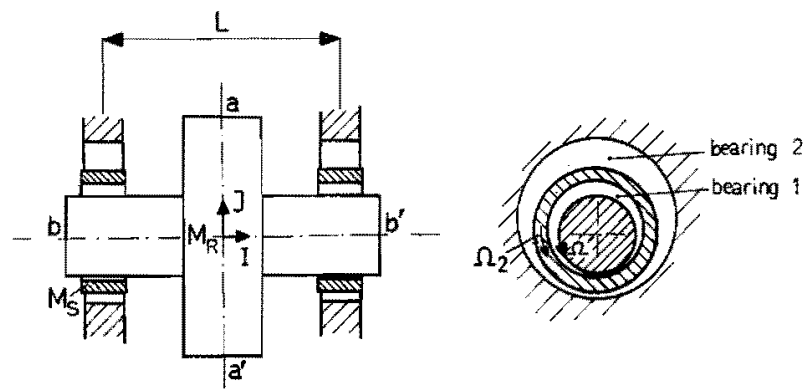

Fig. 4.15. Rotor with floating-bush bearings.

Here $M_{R}$ is the mass of the rotor and $M_{S}$ that of the bush: $I$ is the moment of inertia about $\mathrm{bb}^{\prime}$ and $J$ that about aa'; $\Omega$ is the angular velocity of the rotor and $\Omega_{2}$ that of the bush.

We start from the following assumptions:

- The construction and the external load are symmetrical with respect to aa'.

- The rotor is rigid and has rotational symmetry with respect to $b b^{\prime}$.

- The external load is constant.

- The bearing reactions are linear functions, with constant coefficients, of the relative displacements $\mathbf{X}_{b}$ and $\mathbf{X}_{s}$ and of the velocities $(\mathrm{d} / \mathrm{d} t) \mathbf{X}_{b}$ and $(\mathrm{d} / \mathrm{d} t) \mathbf{X}_{s}$ (see fig. 4.4).

The coordinates of the centres of the journals can again be represented, in fig. 4.4, by $\mathrm{O}_{E}$ and $\mathrm{Q}_{\mathrm{E}}$ in the equilibrium position and by $\mathrm{O}$ and $\mathrm{Q}$ after a displacement from the equilibrium position.

The motion of this system is described by the following dimensionless equations (cf. sec. 4.3.1).

Journal forces

$$
\begin{aligned}
& \Delta f_{b x}=-a_{b x x} x_{b}-\frac{1}{1+\nu} b_{b x x} \dot{x}_{b}-a_{b x y} y_{b}-\frac{1}{1+\nu} b_{b x y} \dot{y}_{b}, \\
& \Delta f_{b y}=-a_{b y y} y_{b}-\frac{1}{1+\nu} b_{b y y} \dot{y}_{b}-a_{b y x} x_{b}-\frac{1}{1+v} b_{b y x} \dot{x}_{b .} .
\end{aligned}
$$


Forces on bearing bush

$$
\begin{aligned}
& \Delta f_{s x}=-\Delta f_{b x}-v_{c} a_{s x x} x_{s}-\frac{v_{c}}{v} b_{b x x} \dot{x}_{s}-v_{c} a_{s x y} y_{s}-\frac{v_{c}}{v} b_{s x y} \dot{y}_{s,} \\
& \Delta f_{s y}=-\Delta f_{b y}-v_{c} a_{s y y} y_{s}-\frac{v_{c}}{v} b_{b y y} \dot{y}_{s}-v_{c} a_{s y x} x_{s}-\frac{v_{c}}{v} b_{s y x} \dot{y}_{s v}
\end{aligned}
$$

Equations of motion of the rotor

$$
\begin{aligned}
\Delta f_{b x} & =\frac{1}{\bar{W}}(\ddot{x}+\lambda \dot{y}), \\
\Delta f_{b y} & =\frac{1}{\bar{W}}(\ddot{y}-\lambda \dot{x}) .
\end{aligned}
$$

Equations of motion of the bearing bush

$$
\begin{aligned}
\Delta f_{s x} & =\frac{m_{s}}{\mathscr{W}} \ddot{x}_{s}, \\
\Delta f_{s y} & =\frac{m_{s}}{W} \ddot{y}_{s} .
\end{aligned}
$$

The dimensionless quantities are defined as follows:

$$
\begin{aligned}
& \Delta f_{b x}=\frac{\Delta F_{b x}}{F_{01}}, \quad \Delta f_{b y}=\frac{\Delta F_{b y}}{F_{01}}, \quad \text { dynamic parts of the forces } \\
& \Delta f_{s x}=\frac{\Delta F_{s x}}{F_{01}}, \quad \Delta f_{s y}=\frac{\Delta F_{s y}}{F_{01}} \quad \begin{array}{l}
\text { dynamic parts of the forces } \\
\text { acting on the bush, }
\end{array} \\
& x_{b}=\frac{X_{b}}{\Delta R_{1}}, \quad y_{b}=\frac{Y_{b}}{\Delta R_{1}} \quad \text { deflections of the journal, } \\
& x_{s}=\frac{X_{s}}{\Delta R_{1}}, \quad y_{s}=\frac{Y_{s}}{\Delta R_{1}} \quad \text { deflections of the support, } \\
& a_{b x x}=\frac{\Delta R_{1}}{F_{01}} A_{b x x}, \quad \text { etc. } \quad \begin{array}{l}
\text { stiffness coefficients of the } \\
\text { inner bearing, }
\end{array} \\
& b_{b x x}=\frac{\Omega_{1} \Delta R_{1}}{F_{01}} B_{b x x}, \quad \text { etc. } \quad \begin{array}{l}
\text { damping coefficients of the } \\
\text { inner bearing, }
\end{array} \\
& a_{s x x}=\frac{\Delta R_{2}}{F_{02}} A_{s x x}, \quad \text { etc. } \quad \begin{array}{l}
\text { stiffness coefficients of the } \\
\text { outer bearings, }
\end{array} \\
& b_{s x x}=\frac{\Omega_{2} \Delta R_{2}}{F_{02}} B_{s x x} \quad \begin{array}{l}
\text { damping coefficients of the } \\
\text { outer bearing, }
\end{array}
\end{aligned}
$$


$\vec{W}=\left\{\begin{array}{ll}\frac{2 F_{01}}{M_{R} \Delta R_{1} \Omega^{2}} & \text { (translational mode) } \\ \frac{F_{01} L^{2}}{2 J \Delta R_{1} \Omega^{2}} & \text { (conical mode) }\end{array}\right\} \begin{aligned} & \text { stability } \\ & \text { parameter }\end{aligned}$

$\lambda=\left\{\begin{array}{cl}0 & \text { (translational mode) } \\ \frac{I}{J} & \text { (conical mode) }\end{array}\right\} \begin{aligned} & \text { gyroscopic } \\ & \text { parameter }\end{aligned}$

$m_{s}=\left\{\begin{array}{ll}\frac{2 M_{S}}{M_{R}} & \text { (translational mode) } \\ \frac{M_{S} L^{2}}{2 I} & \text { (conical mode) }\end{array}\right\} \begin{aligned} & \text { mass of the } \\ & \text { support, }\end{aligned}$

$x=x_{b}+x_{s}, y=y_{b}+y_{s} \quad$ total deflections of the journal,

$\tau=\Omega t$

dimensionless time.

Further

$F_{01}=\frac{12 \eta_{1} \Omega_{1} R_{1}^{4}}{\left(\Delta R_{1}\right)^{2}} \quad$ teference force 1elating to the inner bearing,

$F_{02}=\frac{12 \eta_{2} \Omega_{2} R_{2}^{4}}{\left(\Delta R_{2}\right)^{2}} \quad$ reference force relating to the outer bearing,

$\Omega_{1}=\Omega+\Omega_{2}$ effective angular velocity of the inner bearing, (4.36p)

$Q_{2}$ effective angular velocity of the outer bearing, (4.36q)

$v=\frac{\Omega_{2}}{\Omega}$

$v_{c}=\frac{F_{02}}{F_{01}} \frac{\Delta R_{1}}{\Delta R_{2}}$

If, analogously to sec. 4.33 , we put $m_{s}=\lambda=0$, the characteristic determinant and characteristic equation are given again by (4.14) and, instead of (4.15), we obtain 


$$
\begin{aligned}
& a_{11}=\mathscr{W} a_{b x x}, \\
& b_{11}=\bar{W} \frac{1}{1+v} b_{b x x}, \\
& a_{12}=\bar{W} a_{b x y} \\
& b_{12}=\bar{W} \frac{1}{1+v} b_{b x y}, \\
& a_{21}=\bar{W} a_{b y x}, \\
& b_{21}=W \frac{1}{1+v} b_{b y x}, \\
& a_{22}=\mathscr{W} a_{b y y}, \\
& b_{2 z}=\bar{W} \frac{1}{1+v} b_{b y y} ; \\
& a_{33}=\bar{W} v_{c} a_{s x x} \\
& b_{33}=W \frac{v_{c}}{v} b_{s x x}, \\
& a_{34}=\bar{W} v_{c} a_{s x y}, \\
& b_{34}=\bar{W}_{\frac{\nu_{c}}{v}} b_{s x y}, \\
& a_{43}=\bar{W} v_{c} a_{s y x}, \\
& b_{43}=W^{\nu_{c}} \frac{b_{s y x}}{y}, \\
& a_{44}=\mathscr{W} v_{c} a_{s y y}, \\
& b_{44}=\bar{W} \frac{\nu_{c}}{v} b_{s y v} .
\end{aligned}
$$

4.5.2. Approximate torque balance for the determination of the angular velocity of the bush

For a given construction the angular velocity of the bush, $\Omega_{2}$, determines the stiffness ratio of the inner to the outer bearing.

Figure 4.16 shows a concentric floating-bush bearing. In this case, with smooth bearings, the torques transmitted by the lubricating films are:
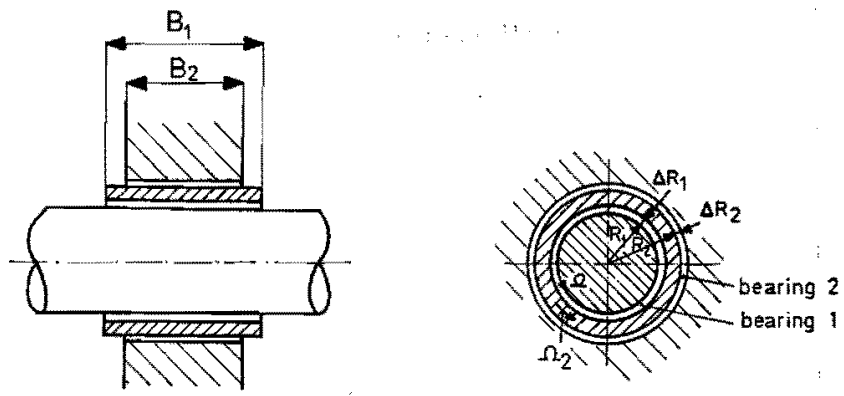

Fig. 4.16. Floating bush bearing in the concentric position. 
inner bearing:

$$
T_{1}=2 \pi \eta_{1} \frac{R_{1}^{3} B_{1}}{\Delta R_{1}}\left(\Omega-\Omega_{2}\right)
$$

outer bearing: $\quad T_{2}=2 \pi \eta_{2} \frac{R_{2}{ }^{3} B_{2}}{\Delta R_{2}} \Omega_{2}$.

If the torques $T_{1}$ and $T_{2}$ alone act on the bush and if we assume that the above formulae also yield a reasonable approximation for eccentric and grooved bearings, the speed of the bush can be obtained from (4.38) by putting $T_{1} \approx T_{2}$ :

$$
\begin{gathered}
\frac{\Omega-\Omega_{2}}{\Omega_{2}}=\left(\frac{R_{2}}{R_{1}}\right)^{3} \frac{B_{2}}{B_{1}} \frac{\Delta R_{1}}{\Delta R_{2}} \frac{\eta_{2}}{\eta_{1}}, \\
\frac{1}{y}=1+\left(\frac{R_{2}}{R_{1}}\right)^{3} \frac{B_{2}}{B_{1}} \frac{\Delta R_{1}}{\Delta R_{2}} \frac{\eta_{2}}{\eta_{1}} .
\end{gathered}
$$

Further

$$
\begin{aligned}
& v_{c}=\frac{v}{1+v} \frac{\eta_{2}}{\eta_{1}}\left(\frac{R_{2}}{R_{1}}\right)^{4}\left(\frac{\Delta R_{1}}{\Delta R_{2}}\right)^{3}, \\
& \breve{W}= \begin{cases}\frac{24 \eta_{1} R_{1}{ }^{4}}{M_{R} \Omega\left(\Delta R_{1}\right)^{3}}(1+v) & \text { (translational mode) } \\
\frac{6 \eta_{1} R_{1}{ }^{4} L^{2}}{J \Omega\left(\Delta R_{1}\right)^{3}}(1+v) & \text { (conical mode). }\end{cases}
\end{aligned}
$$

Since $W$ still contains the parameter $v$, we cannot yet interpret $W$ as the reciprocal of the speed. We therefore introduce

$$
\bar{W}^{*}= \begin{cases}\frac{24 \eta_{1} R_{1}{ }^{4}}{M_{R} \Omega\left(\Delta R_{1}\right)^{3}} & \text { (translational mode) } \\ \frac{6 \eta_{1} R_{1}{ }^{4} L^{2}}{J \Omega\left(\Delta R_{1}\right)^{3}} & \text { (conical mode) }\end{cases}
$$

so that $W^{*}$ now represents the reciprocal of the speed of the rotor.

\subsubsection{Application to ORS bearings}

The stability diagram of fig. 4.17 gives the location of the stable and unstable regions for a rotor in floating-bush bearings as a function of $W^{*}$ and $\Delta R_{1} / \Delta R_{2}$. The floating-bush bearings are built up from two ORS bearings, with $B / D=1$ and $\eta_{1}=\eta_{2}, B_{1}=B_{2}, R_{1} \approx R_{2}$.

The figure shows that the stability of the rotor is optimum for $\Delta R_{1} / \Delta R_{2}=$ 


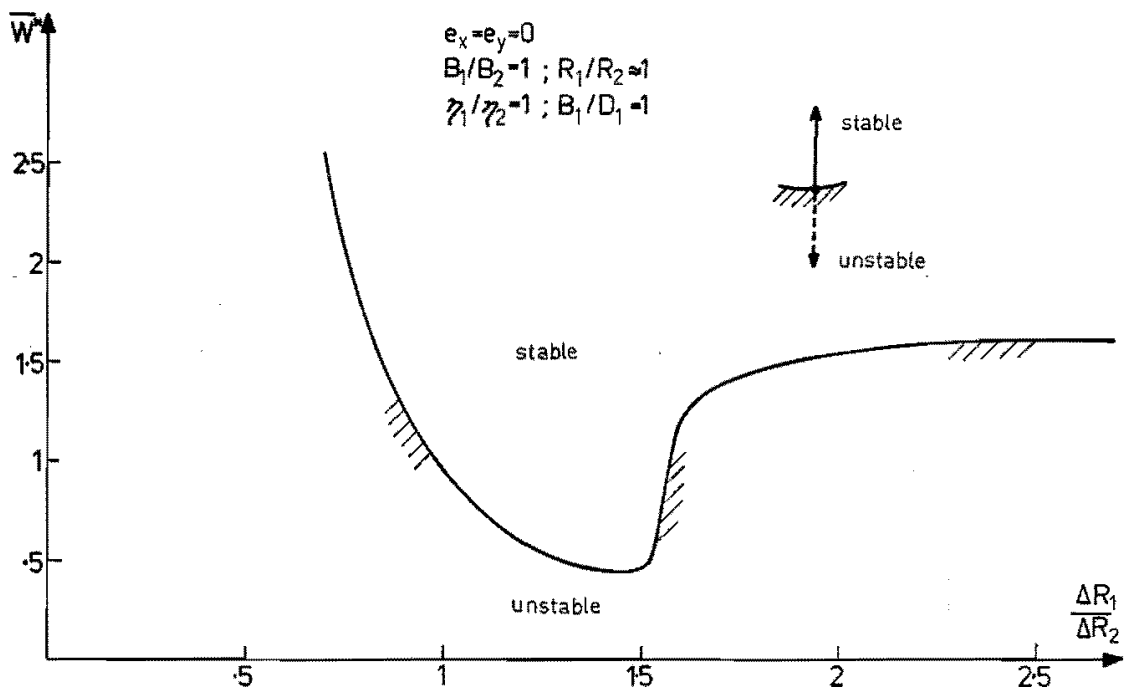

Fig. 4.17. Stability curve of a rotor with floating-bush bearing (torque balanced); inner bearing: ORS $(B / D=1)$; outer bearing: ORS $(B / D=1)$.

1.4. The poor stability at low values of $\Delta R_{1} / \Delta R_{2}$ is due to the fact that the diagram really indicates the stability as a function of $\Delta R_{2}$ and of the reciprocal of the speed, the other parameters being considered as constants: a small value of $\Delta R_{1} / \Delta R_{2}$ implies a large $\Delta R_{2}$, leading to slight stiffness and slight damping of the outer bearing, so that on the basis of the previous results, the stability will be poor.

\subsubsection{Floating-bush bearing with a freely chosen bush speed}

In the preceding section the velocity of the bush was governed by the balance of torques.

In fig. 4.17 this means that the stiffness and the damping of the outer bearing are fixed for a certain value of $\Delta R_{1} / \Delta R_{2}$. We have seen, with the system of the flexible support with springs and dampers in sec. 4.2 , that in particular the appropriate combination of stiffness and damping of the support yields optimum stability. Therefore the coupling of damping and stiffness by the balance of torques can have an unfavourable effect on attempts to reach optimum stability.

Hence for the rotor in the preceding section the stability has been investigated with $\Omega_{2} / \Omega$ as a freely adjustable parameter between the limits of 0 and 1 . The result is given in fig. 4.18, which also shows the curve for $\left(\Omega_{2} / \Omega\right)_{k}$, representing the value prescribed on the basis of the balance of torques. This figure clearly reveals that, especially at low values of $\Omega_{2} / \Omega$ (substantially lower than those obtained from the balance of torques) a satisfactory stability can be reached in the range of ca $0.9<\left(\Delta R_{1} / \Delta R_{2}\right)<$ ca 1.4 . 


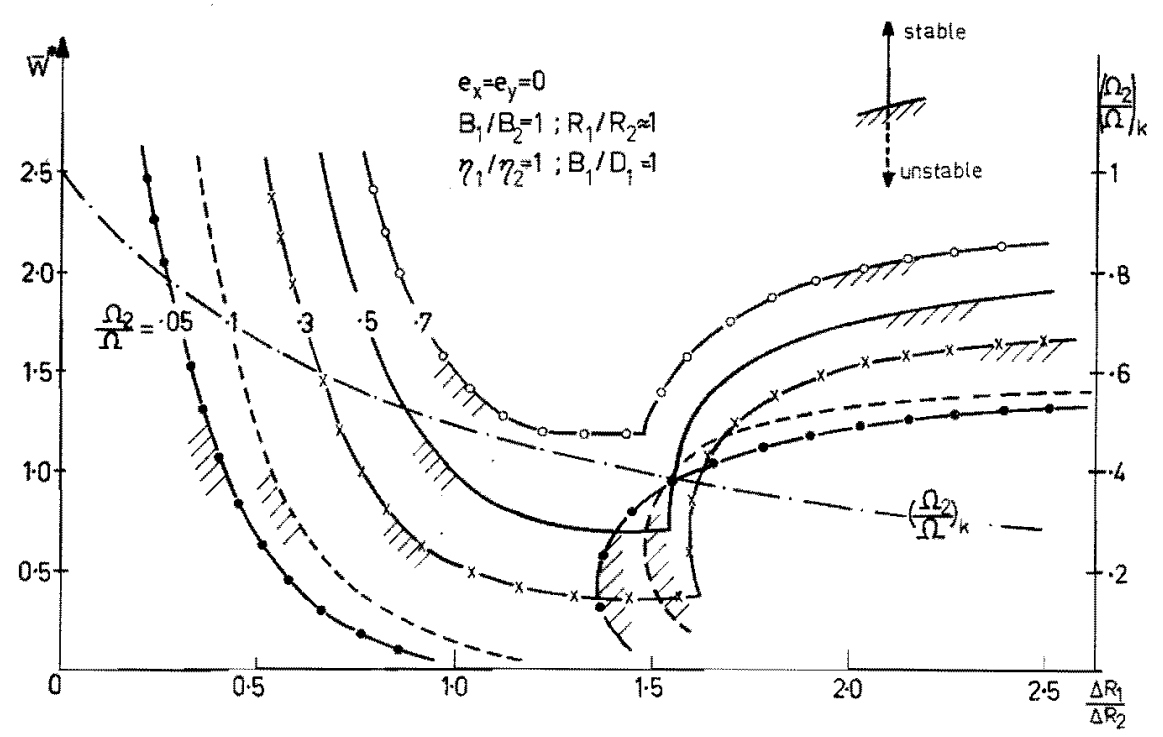

Fig. 4.18. Stability curves of a rotor with floating-bush bearing for various bush speeds; inner bearing: $\mathrm{ORS}(B / D=1)$; outer bearing: ORS $(B / D=1)$.

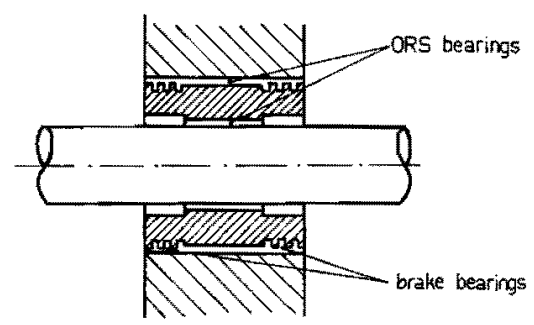

Fig. 4.19. Floating-bush bearing with additional brake bearings for various bush speeds.

Low values of $\Omega_{2} / \Omega$ are well realizable in practice by artificial braking of the motion of the bush. A possible design is displayed in fig. 4.19. Mounted on both sides of the normal bearings there are bearings which are responsible solely for a reduction of the speed of the bush, without their contributing much to the load-carrying capacity (they have therefore been given grooves).

\subsubsection{Floating-bush bearing with smooth bearings}

To smooth bearings, under no-load conditions, the relation

$$
a_{x x}=a_{y y}=0
$$

applies. This is to say that these bearings have no radial stiffness at all. By analogy with the preceding bearings, a few smooth floating-ring bearings have been investigated with respect to their stability. It has been found that these bearings are inherently unstable under no-load conditions. 
Obviously, the good stability reported by Orcutt and $\mathrm{Ng}^{4-4}$ ) cannot be explained by means of a linear theory.

Analogously to the nonlinear theory, described in chapter 3, it is also quite possible here that with a floating-bush bearing which was initially unstable, a certain deflection leads to such a great radial load-carrying capacity that the bearing and the bush are capable of describing stable circles. Some evidence of this has been obtained also from exploratory calculations, but these will not be dealt with here.

\section{REFERENCES}

4-1) J. Kerr, The onset and cessation of half-speed whirl in air-lubricated self pressurised journal bearings, Proc. Inst. mech. Engrs 180, part 3k, 145-153, 1965/66.

4-2) H. Marsh, The stability of self acting gas journal bearings with noncircular members and additional elements of flexibility, Lub. Symposium, Las Vegas (1968) paper no. 68-LubS-45.

4-3) J. W. Powell and M. C. Tempest, A study of high speed machines with rubber stabilized air bearings, Trans. ASME, J. Lub. Techn. 90, 701-708, 1968.

4-4) F. K. Orcutt and C. W. Ng, The steady-state and dynamic characteristics of the floating-ring journal bearing, Trans. ASME, J. Lub. Techn. 90, 243-253, 1968.

4-5) J. W. Lund, The stability of an elastic rotor in journal bearings with flexible, damped supports, Trans. ASME, J. appl. Mech. 32, 911-920, 1965.

4-6) A. Tond1, The effect of an elastically suspended foundation mass and its damping on the initiation of self-excited vibrations of a rotor mounted in air pressurised bearings, Gas Bearing Symposium (Univ. Southampton 1971), vol. 1, paper no. 1.

${ }^{4-7}$ ) H. Mori and A. Mori, A stabilizing method of the externally pressurized gas journal bearings, Gas Bearing Symposium (Univ. Southampton, 1969), vol. 2, paper no. 29.

4-8) H. Mori and A. Mori, A stabilizing method of the externally pressurized gas journal bearings, Gas Bearing Symposium (Univ. Southampton, 1971), vol. 1, paper no. 4. 


\section{THE FINITE-ELEMENT METHOD FOR THE CALCULATION OF SLIDING BEARINGS}

\subsection{Introduction}

If one wants to investigate the dynamic behaviour of a rotor-and-bearing system, then one must, in addition to the equations of motion of the rotor, know the dynamic properties of the bearings used. For sliding bearings these properties can in principle be determined by solving the Reynolds differential equation, which describes the pressure distribution of the lubricant in the film gap. Since an exact solution has proved to be possible in only a few cases, mainly approximate methods have to be used. In such a method, use is often made of calculation programs based on the well-known finite-difference method $\left.^{5-10}\right)$.

At present the number of known types of sliding bearings (smooth bearings, grooved bearings; cylindrical, conical and spherical bearings; hydrodynamic and hydrostatic bearings; etc.), which frequently possess very different geometries, is so large that it is desirable to have the disposal of a calculation program which enables one to compute all these types of bearings. Hence this calculation program must be versatile. The versatility becomes even more important if one wants to examine, for example in the design phase of a rotorand-bearing system, the effect of a geometric modification, such as displacement of a lubricant groove to another place.

A method which possesses this versatility to a high degree is that which is known as the Finite Element Method (FEM). This method is currently employed on a wide scale, for instance in calculations of the strength of constructions. It will become apparent from the present chapter that the finite-element principle is applicable to the calculation of sliding bearings, too.

The method takes its name from the fact that the region in which the differential equation has to be solved is divided into a number of sub-regions known as elements. In these elements the function to be calculated is greatly simplified, and one restricts oneself to solutions that are uniquely defined by the functional values in the "nodal points" of the element. In order to find the approximate solution for the whole region one does not employ the Reynolds equation in its original form but transforms it into a specific integral known as a variation integral. The function of the solution which minimises this integral is adopted as the "best approximation".

In this chapter the variation integral associated with the Reynolds equation will be derived. Minimisation leads to a set of linear equations which describe the solution, viz. the pressures in the nodal points. Next, for grooved bearings, a derivation is given of a "generalised" Reynolds differential equation for the "smoothed pressure", which means that the local pressure fluctuations above 
the grooves and ridges are smoothed. Again, by a procedure similar to that for the "ordinary" Reynolds equation, the variation integral and the set of linear equations for the nodal pressures are found.

In sec. 5.5 a triangular element with a linear pressure distribution will be discussed by way of an example.

Besseling ${ }^{5-1}$ ) and Zienckiewicz ${ }^{5-2}$ ) have pointed out that the FEM is generally applicable and that, in addition to strength calculations of constructions, it can be applied also to problems of heat conduction. Reddi, for example, used the method for the incompressible ${ }^{5-3}$ ) and the compressible ${ }^{5-4}$ ) Reynolds equations. Argyris and Scharpf ${ }^{5-5}$ ), also, described a theory for the incompressible Reynolds equation. Vogelpohl ${ }^{5-6}$ ) was probably the first to use the variation principle for the calculation of sliding bearings.

The derivation presented here, which differs from those of the authors just referred to, is particularly useful for a clear insight into the proper approach and the way in which the boundary conditions must be handled.

Hirs ${ }^{5-7}$ ), and Vohr and Pan ${ }^{5-8}$ ) used the principle of the smoothed pressure in the calculation of grooved bearings. They assumed the number of grooves to be infinite, but this turns out to be an unnecessary limitation.

The combination of the FEM and the generalised Reynolds equation for grooved bearings facilitates the application considerably; it does not, however, seem to have been published previously.

\subsection{Principles of the method}

One of the advantages of the FEM is that it makes a strong appeal to the physical sense of the user. Because of this, errors can be avoided, and when applying the method one realises where the inaccuracies arise. Before a beginning is made with the derivations it is therefore relevant to outline the principles used for the case of incompressible flow under consideration.

In a calculation of a bearing the problem is to find that function, the pressure $P\left(X_{f}, Y_{f}\right)$, which in the region $G$ (fig. 5.1a) obeys the Reynolds differential equation

$$
f\left(\frac{\partial^{2} P}{\partial X_{f}^{2}}, \frac{\partial P}{\partial X_{f}}, \ldots, X_{f}, Y_{f}\right)=0
$$

and satisfies the conditions at the boundary $R$. If the principles of variation calculus are used, this can also be formulated in terms of finding the function $P\left(X_{f}, Y_{f}\right)$ which minimises the variation integral

$$
\Phi=\int_{G} g\left(\frac{\partial P}{\partial X_{f}}, P, \ldots, X_{f}, Y_{f}\right) \mathrm{d} G .
$$


a)

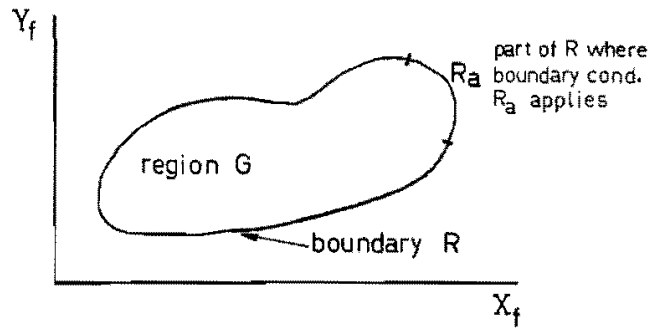

b)

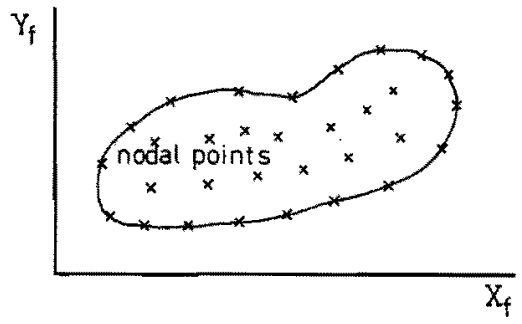

c)

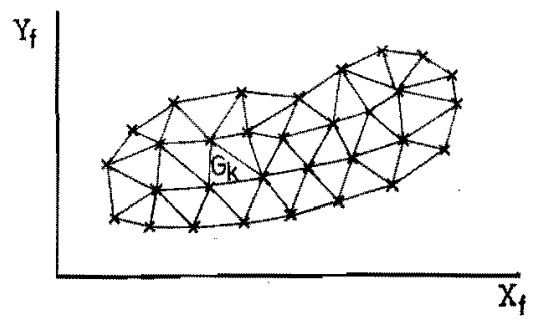

Fig. 5.1. The steps taken in the FEM.

In looking for the minimum of $\Phi$ we might in principle vary the pressure $P$ in every point of the region $G$, until the minimum is found. To this end we have to vary the pressure in " $\infty$ " " points of $G$.

The first step taken in the FEM is that, in determining the minimum, one contents oneself with varying the pressure in a finite number of points, the nodal points of $G$, and specifying the pressure between the nodal points (fig. $5.1 b$ ) by means of interpolation functions. Here we shall not go into the conditions to be fulfilled by the interpolation functions but confine ourselves to the remark that in $G$ the integrand must be sufficiently "smooth", so that by increasing the number of nodal points we are able to approximate the exact solution to an arbitrarily close degree (cf. ref. 5-2).

The second step to be taken consists of dividing $G$ into sub-regions (the elements $G_{k}$ ) and determining the pressure distribution within these elements by means of the associated nodal points and interpolation functions (fig. 5.1c). A refinement of the distribution of elements increases the number of nodal point and thus improves the accuracy. 
The choice of the elements and associated interpolation functions is governed by the expected pressure distribution, which must admit of being represented sufficiently accurately by means of available degrees of freedom (the pressures in the nodal points). If, after a preliminary calculation it turns out that this has not been the case, then it is possible to perform further calculations with adapted element distributions.

\subsection{The finite-element method based on the "ordinary" Reynolds equation}

\subsubsection{The Reynolds differential equation}

Figure 5.2 shows an element $A B C D A^{\prime} B^{\prime} C^{\prime} D^{\prime}$ of a liquid film. Here $A B C D$ is a part of the stationary surface and $A^{\prime} B^{\prime} C^{\prime} D^{\prime}$ a part of the moving surface. It is assumed that the flow is perfectly viscous and that the density and viscosity are constant.

Using the Navier-Stokes equations, with the usual simplifications (Cameron $\left.{ }^{5-9}\right)$ ), it then follows for the sub-element $a b c d a^{\prime} b^{\prime} c^{\prime} d^{\prime}$ that

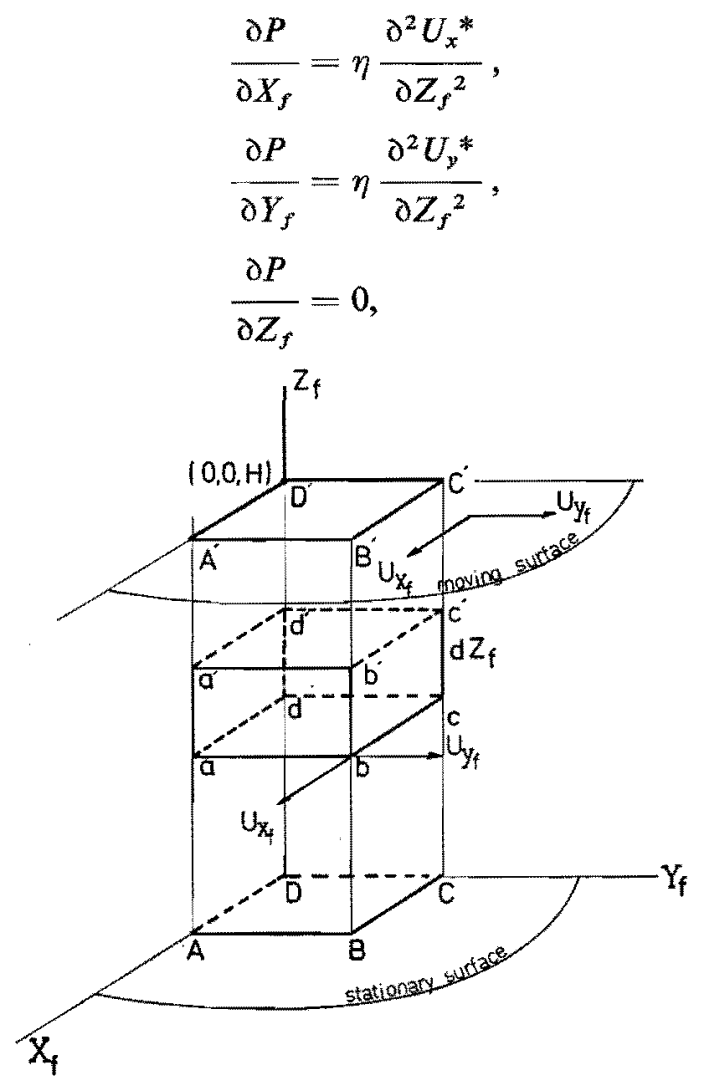

Fig. 5.2. Element of the lubricant film. $H$ is the thickness of the lubricant film at the location of element $A B C D A^{\prime} B^{\prime} C^{\prime} D^{\prime}$. 
where $P$ is the pressure and $U_{x}{ }^{*}$ and $U_{y}{ }^{*}$ are velocities in respectively the $X_{j}$ - and $Y_{j}$-directions.

With

$$
\begin{aligned}
& U_{x}^{*}\left(Z_{f}=H\right)=U_{x}, \\
& U_{y}{ }^{*}\left(Z_{f}=H\right)=U_{y}, \\
& U_{z}^{*}\left(Z_{f}=0\right)=0, \\
& U_{y}{ }^{*}\left(Z_{f}=0\right)=0,
\end{aligned}
$$

and

$$
\begin{aligned}
& Q_{x}=\int_{0}^{H} U_{x}^{*} \mathrm{~d} Z_{f}, \\
& Q_{y}=\int_{0}^{H} U_{y}{ }^{*} \mathrm{~d} Z_{f},
\end{aligned}
$$

it follows from (5.1) that

$$
\begin{aligned}
& Q_{x}=-\frac{H^{3}}{12 \eta} \frac{\partial P}{\partial X_{f}}+\frac{H U_{x}}{2}, \\
& Q_{y}=-\frac{H^{3}}{12 \eta} \frac{\partial P}{\partial Y_{f}}+\frac{H U_{y}}{2} .
\end{aligned}
$$

To the element $A B C D A^{\prime} B^{\prime} C^{\prime} D^{\prime}$ the equation for continuity is applicable in the following form:

$$
\frac{\partial Q_{x}}{\partial X_{f}}+\frac{\partial Q_{y}}{\partial Y_{f}}+\frac{\partial H}{\partial t}=0 .
$$

Now dimensionless quantities are introduced as follows:

$$
\begin{aligned}
q_{x} & =\frac{Q_{x}}{Q_{0}} ; \quad q_{y}=\frac{Q_{y}}{Q_{0}} ; \\
u_{x} & =\frac{U_{x}}{\Omega_{0} L_{0}} ; u_{y}=\frac{U_{y}}{\Omega_{0} L_{0}} ; \\
x_{f} & =\frac{X_{f}}{L_{0}} ; \quad y_{f}=\frac{Y_{f}}{L_{0}} ; \\
h & =\frac{H}{H_{0}} ; \\
\tau & =\Omega_{0} t ; \\
p & =\frac{P}{P_{0}} .
\end{aligned}
$$


Here $Q_{0}$ (the reference flow), $H_{0}$ (the reference film thickness), $L_{0}$ (the reference length), $\Omega_{0}$ (the reference angular velocity), and $P_{0}$ (the reference pressure) can still be chosen freely.

Equations (5.4) and (5.5) can now be rewritten as

$$
\begin{aligned}
& \frac{Q_{0}}{\Omega_{0} H_{0} L_{0}} q_{x}=-\frac{H_{0}^{2} P_{0}}{12 \eta \Omega_{0} L_{0}^{2}} h^{3} \frac{\partial p}{\partial x_{f}}+\frac{1}{2} h u_{x}, \\
& \frac{Q_{0}}{\Omega_{0} H_{0} L_{0}} q_{y}=-\frac{H_{0}^{2} P_{0}}{12 \eta \Omega_{0} L_{0}^{2}} h^{3} \frac{\partial p}{\partial y_{f}}+\frac{1}{2} h u_{y} .
\end{aligned}
$$

If we now choose

$$
\begin{aligned}
& Q_{0}=\Omega_{0} H_{0} L_{0}, \\
& P_{0}=\frac{12 \eta \Omega_{0} L_{0}^{2}}{H_{0}^{2}}
\end{aligned}
$$

and pass into vector notation we may write:

$$
\begin{gathered}
\mathbf{q}=-h^{3} \nabla p+\frac{1}{2} h \mathbf{u}, \\
(\nabla \cdot \mathbf{q})+\frac{\partial h}{\partial \tau}=0 .
\end{gathered}
$$

The relations (5.10) and (5.11) hold for any coordinate system. Eliminating the flow $\mathbf{q}$ from these equations one obtains the dimensionless Reynolds equation:

$$
\left(\nabla \cdot\left(h^{3} \nabla p\right)\right)-\frac{1}{2}(\nabla \cdot(h \mathbf{u}))-\frac{\partial h}{\partial \tau}=0 .
$$

\subsubsection{Transformation of the Reynolds equation into a variation integral}

The calculation of the pressure distribution in a hydrodynamic or hydrostatic bearing amounts to the solution of the Reynolds differential equation (5.12) in a region $G$ with prescribed boundary conditions at its boundary $R$. It will be assumed that there will be a solution, $p$, with its derivatives, in the region $G$. Then it follows from the principles of variation calculus (cf. Courant ${ }^{5-12}$ )) that the relation

$$
\delta \Phi=0=\int_{G}\left(\left(\nabla \cdot\left(h^{3} \nabla p\right)\right)-\frac{1}{2}(\nabla \cdot(h \mathbf{u}))-\frac{\partial h}{\partial \tau}\right) \delta p \mathrm{~d} G
$$

is equivalent to (5.12). 
For the following operations all that is required with respect to the variation of the pressure, $\delta p$, is that it can be sufficiently frequently differentiated in $G$; other restrictions, imposed on $\delta p$ by the boundary conditions, may be introduced during the final stage of the solution of the problem.

Using Gauss' theorem we may convert (5.13) as follows:

$$
\begin{aligned}
\int_{G}\left(\nabla \cdot\left(h^{3} \nabla p\right)\right) \delta p \mathrm{~d} G & =\int_{G}\left(\nabla \cdot\left(h^{3} \delta p \nabla p\right)\right) \mathrm{d} G-\int_{G}\left(h^{3} \nabla p \cdot \nabla \delta p\right) \mathrm{d} G \\
& =\int_{\boldsymbol{R}}\left(h^{3} \nabla p \cdot \mathbf{n}\right) \delta p \mathrm{~d} R-\delta \int_{G}\left(\frac{1}{2} h^{3} \nabla p \cdot \nabla p\right) \mathrm{d} G \\
-\frac{1}{2} \int_{G}(\nabla \cdot(h \mathbf{u})) \delta p \mathrm{~d} G & =-\frac{1}{2} \int_{G}(\nabla \cdot(h \delta p \mathbf{u})) \mathrm{d} G+\frac{1}{2} \int_{G}(h \mathbf{u} \cdot \nabla \delta p) \mathrm{d} G \\
& =-\frac{1}{2} \int_{R}(h \mathbf{u} \cdot \mathbf{n}) \delta p \mathrm{~d} R+\frac{1}{2} \delta \int_{G}(h \mathbf{u} \cdot \nabla p) \mathrm{d} G \\
-\int_{G} \frac{\partial h}{\partial \tau} \delta p \mathrm{~d} G & =-\delta \int_{G} \frac{\partial h}{\partial \tau} p \mathrm{~d} G
\end{aligned}
$$

then (5.13) becomes:

$$
\begin{aligned}
\delta \Phi=0= & -\delta \int_{G}\left(\frac{h^{3}}{2} \nabla p \cdot \nabla p-\frac{h}{2} \mathbf{u} \cdot \nabla p+\frac{\partial h}{\partial \tau} p\right) \mathrm{d} G+ \\
& -\int_{\boldsymbol{R}}\left(\left(-h^{3} \nabla p+\frac{h}{2} \mathbf{u}\right) \cdot \mathbf{n}\right) \delta p \mathrm{~d} R .
\end{aligned}
$$

Here $\mathbf{n}$ is a vector with unit length normal to the boundary $R$ and directed outwards with respect to $G$.

In (5.17)

$$
\int_{G}\left(\frac{h^{3}}{2} \nabla p \cdot \nabla p-\frac{h}{2} \mathbf{u} \cdot \nabla p+\frac{\partial h}{\partial \tau} p\right) \mathrm{d} G
$$

is the variation integral. The region over which the integral is calculated may be multicoherent. If this region is not coherent, the problem may be divided in part problems.

\subsubsection{Boundary conditions}

An important point to investigate is how different boundary conditions can be applied to (5.17). For this purpose let us consider the second term of the right-hand side of that equation, meanwhile defining $\Phi_{\text {boundary }}$ by means of 


$$
\delta \Phi_{\text {boundary }}=\int_{\boldsymbol{R}}\left(\left(-h^{3} \nabla p+\frac{h \mathbf{u}}{2}\right) \cdot \mathbf{n}\right) \delta p \mathrm{~d} R .
$$

Practical boundary conditions that may occur in different problems are the following.

\section{Boundary condition $R_{a}$}

The pressure at a section $R_{a}$ of the boundary is required to have an prescribed value. Since $\delta p$ is not permitted to affect the boundary conditions, the relation $\delta p=0$ should hold at this boundary:

$$
\delta \Phi_{\text {boundary }}=\delta \Phi_{R_{a}}=0 .
$$

Boundary condition $R_{b}$

The outflow at a section $R_{b}$ of the boundary has a prescribed value. This outflow per unit length is

$$
q_{n}=\left(-h^{3} \nabla p+\frac{h \mathbf{u}}{2}\right) \cdot \mathbf{n} .
$$

Hence, at this boundary, it is true that

$$
\delta \Phi_{\text {boundary }}=\int_{R_{b}} q_{n} \delta p \mathrm{~d} R=\delta \int_{R_{b}} q_{n} p \mathrm{~d} R .
$$

An alternative is that the total flow, $q_{\mathrm{tot} n}$ across the section of the boundary $R_{b}$ is prescribed, it being required that the pressure is uniform everywhere at this boundary $\left(=p_{r b}\right)$. We may then write:

$$
\delta \Phi_{\text {boundary }}=\int_{R_{b}} q_{n} \delta p \mathrm{~d} R=q_{\text {tot } n} \delta p_{r b} .
$$

\section{Boundary condition $R_{c}$}

For part $R_{c 1}$ of the boundary it is required that the pressure there is equal to that at part $R_{c 2}$, and that the normal outflow at $R_{c 1}$ is equal to the inflow at $R_{c 2}$ (cf. fig. 5.3). This boundary condition is obtained if, for instance, a cylindrical surface is cut open and flattened. Then we have:

$$
\delta \Phi_{\text {boundary }}=\int_{R_{c 1}} q_{n} \delta p \mathrm{~d} R+\int_{R_{c 2}} q_{n} \delta p \mathrm{~d} R=0 .
$$

\section{Boundary condition $R_{d}$}

For a section $R_{d}$ of the boundary $R$ it is required that the normal flow across this section of the boundary is proportional to the pressure on that section. Hence we may write: 


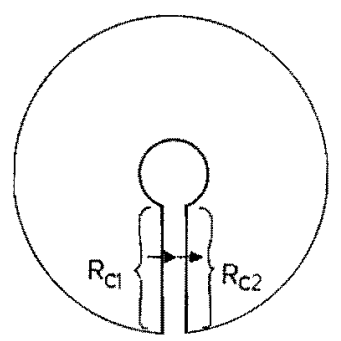

Fig. 5.3. Boundary condition $R_{c}$,

$$
q_{n}=\lambda_{b} p
$$

Therefore

$$
\delta \Phi_{\text {boundary }}=\int_{R_{d}} q_{n} \delta p \mathrm{~d} R=\frac{1}{2} \delta \int_{R_{d}} \lambda_{b} p^{2} \mathrm{~d} R .
$$

The restrictors in hydrostatic bearings may lead to such a boundary condition.

\subsubsection{Introduction of the finite elements}

With (5.17) the integrals of the right-hand side of the equation are evaluated both over the whole region $G$ and over the boundary $R$. We now split up (5.17) into integrals referring to sub-regions (the elements). Let $m$ be the number of elements $G_{k}$ (with boundaries $R_{k}$ ); then we may write instead of (5.17):

$$
\begin{aligned}
\delta \Phi=0= & -\delta \sum_{k=1}^{m} \int_{\mathbf{G}_{k}}\left(\frac{h^{3}}{2} \nabla p \cdot \nabla p-\frac{h}{2} \mathbf{u} \cdot \nabla p+\frac{\partial h}{\partial \tau} p\right) \mathrm{d} G_{k}+ \\
& -\sum_{k=1}^{m} \int_{R_{k}}\left(\left(-h^{3} \nabla p+\frac{h}{2} \mathbf{u}\right) \cdot \mathbf{n}\right) \delta p \mathrm{~d} R_{k} .
\end{aligned}
$$

Within $G$ the boundary integrals of two adjacent elements will cancel each other, and the sum of boundary integrals will furnish a contribution only if the boundary $R_{k}$ forms part of $R\left(R_{k} \in R\right)$. Because (5.19) is equivalent to (5.13) it still contains the exact solution.

At this point we start the approximations and apply the finite element principle. This means that we restrict the solution of (5.19) to those functions, $p$, which are completely defined for each element by the values they assume in the nodal points of this element, the region between the nodal points being described by means of prescribed interpolation functions. The choice of the elements and interpolation functions depends on the nature of the problem to be solved. The interpolation functions should be such that $p$ and its derivation exist in the 
sub-regions $G_{k}$ and that the boundary integrals of two adjacent elements cancel each other.

For the pressure in the $k$ th element we put

$$
p=p\left(x_{f}, y_{f}\right)=\sum_{i=1}^{n} f_{k i} p_{i}=\left\{f_{k}\right\}^{T}\{p\}
$$

Here the summation involves the total number of nodal points $n$ in $G$. In conformity with the requirement that the pressure in $G_{k}$ is governed solely by the pressure in the nodal points of $G_{k}$, it must be true in respect of the interpolations functions that

$$
f_{k i}=0 \quad \text { for nodal points: } \quad i \in G_{k},
$$

and that

$$
\left\{f_{k}\right\}^{T}=\left[0,0, \ldots, 0, f_{k r}, 0, \ldots, 0, f_{k s}, 0, \ldots, 0, f_{k t}, 0, \ldots\right]
$$

is the $1 \times n$ matrix of the interpolation functions for the pressure in element $G_{k}$ with nodal points $r, s$ and $t$.

Furthermore

$$
\{p\}=\left\{\begin{array}{c}
p_{1} \\
p_{2} \\
\vdots \\
p_{n}
\end{array}\right\}
$$

is the $n \times 1$ column matrix of the nodal pressures.

For the pressure gradient $\nabla p$ in $G_{k}$ it follows from (5.20) that

$$
\{\nabla p\}=\left\{\begin{array}{l}
\frac{\partial p}{\partial x_{f}} \\
\frac{\partial p}{\partial y_{f}}
\end{array}\right\}=\left[r_{k}\right]\{p\}
$$

in which

$$
\left[r_{k}\right]=\left[\begin{array}{c}
0,0, \ldots, \frac{\partial f_{k r}}{\partial x_{f}}, \ldots, \frac{\partial f_{k s}}{\partial x_{f}}, \ldots, \frac{\partial f_{k t}}{\partial x_{f}}, \ldots, 0, \ldots \\
0,0, \ldots, \frac{\partial f_{k r}}{\partial y_{f}}, \ldots, \frac{\partial f_{k s}}{\partial y_{f}}, \ldots, \frac{\partial f_{k t}}{\partial y_{f}}, \ldots, 0, \ldots
\end{array}\right]
$$

is the $2 \times n$ matrix of the interpolation functions for the pressure gradient in $G_{k}$. From (5.19), (5.20) and (5.21) it follows that 


$$
\begin{aligned}
\delta \Phi= & -\delta \sum_{k=1}^{m} \int_{G_{k}}\left(\frac{h^{3}}{2}\{p\}^{T}\left[r_{k}\right]^{T}\left[r_{k}\right]\{p\}-\frac{h}{2}\{u\}^{T}\left[r_{k}\right]\{p\}+\frac{\partial h}{\partial \tau}\left\{f_{k}\right\}^{T}\{p\}\right) \mathrm{d} G_{k}+ \\
& -\delta \sum_{k=1}^{m} \int_{R_{k b}} q_{n}\left\{f_{k}\right\}^{T}\{p\} \mathrm{d} R_{k}+ \\
& -\delta \sum_{k=1}^{m} \int_{R_{k d}}^{\lambda} \frac{\lambda}{2}\{p\}^{T}\left\{f_{k}\right\}\left\{f_{k}\right\}^{T}\{p\} \mathrm{d} R_{k}
\end{aligned}
$$

where $R_{k b}$ and $R_{k d}$ denote the sections of the boundary $R$ to which respectively the boundary conditions $R_{b}$ and $R_{d}$ are applicable.

With

$$
\begin{aligned}
& {[a]=\sum_{k=1}^{m} \int_{G_{k}} h^{3}\left[r_{k}\right]^{T}\left[r_{k}\right] \mathrm{d} G_{k},} \\
& \{v\}^{T}=\sum_{k=1}^{m} \int_{G_{k}}^{h} \frac{-}{2}\{u\}^{T}\left[r_{k}\right] \mathrm{d} G_{k}, \\
& \{q\}^{T}=\sum_{k=1}^{m} \int_{G_{k}} \frac{\partial h}{\partial \tau}\left\{f_{k}\right\}^{T} \mathrm{~d} G_{k}, \\
& \{q n\}^{T}=\sum_{k=1}^{m} \int_{R_{k b}} q_{n}\left\{f_{k}\right\}^{T} \mathrm{~d} R_{k}, \\
& {[a l]=\sum_{m}^{m} \int_{R_{k d}} \lambda\left\{f_{k}\right\}\left\{f_{k}\right\}^{T} \mathrm{~d} R_{k},}
\end{aligned}
$$

it follows from (5.22) that

$$
\begin{aligned}
\delta \Phi= & 0=-\delta\left(\frac{1}{2}\{p\}^{T}[a]\{p\}-\{v\}^{T}\{p\}+\{q s\}^{T}\{p\}+\{q n\}^{T}\{p\}+\right. \\
& \left.+\frac{1}{2}\{p\}^{T}[a l]\{p\}\right)= \\
= & -\delta\left(\frac{1}{2}\{p\}^{T}[a]\{p\}-\{p\}^{T}\{v\}+\{p\}^{T}\{q s\}+\{p\}^{T}\{q n\}+\right. \\
& \left.+\frac{1}{2}\{p\}^{T}[a l]\{p\}\right)
\end{aligned}
$$


or

$$
\delta\{p\}^{T}([a]\{p\}-\{v\}+\{q s\}+\{q n\}+[a l]\{p\})=0
$$

\section{Notes}

(1) [a] is symmetrical, since $\left(h^{3}\left[r_{k}\right]^{T}\left[r_{k}\right]\right)^{T}=h^{3}\left[r_{k}\right]^{T}\left[r_{k}\right]$;

$[a l]$ is symmetrical, since $\left(\lambda\left\{f_{k}\right\}\left\{f_{k}\right\}^{T}\right)^{T}=\lambda\left\{f_{k}\right\}\left\{f_{k}\right\}^{T}$;

(2) $[a]$ is positive definite, since $h^{3}(\nabla p \cdot \nabla p) \geqslant 0$;

$[a l]$ is positive definite, since $\lambda(p . p) \geqslant 0$.

As is evident from (5.24a) the whole problem now resolves itself into a variation problem with a finite number of variables, namely the elements of the pressure matrix $\{p\}$. As a consequence of the boundary conditions applicable to the problem, not all the elements of the pressure vector are necessarily unknown, and relationships may exist between the elements of $\{p\}$ and $\delta\{p\}$, etc.

Since the pressure on the boundary is determined via the interpolation functions $f_{k i}$, advantages are offered by expressing a prescribed $q_{n}$ at $R$ by means of the $f_{k i}$ in terms of nodal-point values of these quantities.

A difficulty then arises which is due to the fact that the "influence" of a nodal point extends in general to within the "influence" of other boundary nodal points. The influence of a boundary nodal point in fig. 5.4 extends to within the two boundary elements. This means that the boundary conditions applied to two adjacent boundary nodal points merge into one another, and this presents difficulties in the calculation of (5.23d) and (5.23e) at locations where two sections of the boundary, with different boundary conditions, adjoin each other.

By arranging that the sections of a boundary with different boundary conditions do not adjoin one another but are separated by an element, it is possible to define (5.23d) and (5.23e) uniquely. In fig 5.4, $R_{\mathbf{I}}$ and $R_{I I}$ as well as $R_{\mathrm{V}}$ and $R_{\mathrm{VI}}$ are therefore included when calculating boundary conditions, but $R_{\mathrm{IV}}$ is disregarded.

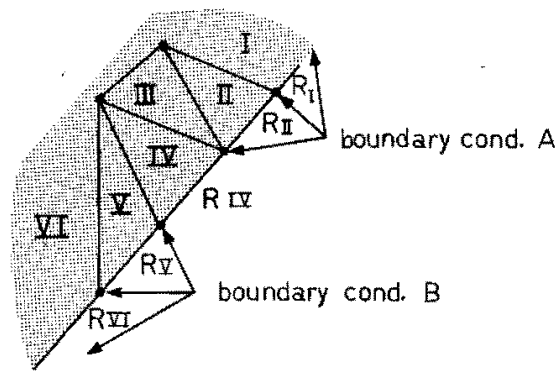

Fig. 5.4. Diffusion of boundary conditions. 


\subsubsection{Application of the boundary conditions}

Let us now examine how the boundary conditions of sec. 5.3 .3 are to be applied to the variation problem given by (5.24a). In doing so, we shall assume the two different sections of the boundary to be separated by an element.

\section{Boundary condition $R_{a}$}

Let $i$ be a nodal point on this boundary, with a given pressure $p_{i}$. Of the pressure variation in this nodal point it is then true to say that

$$
\delta p_{i}=0 \text {. }
$$

Now the term of (5.24a) which corresponds to $\delta p_{i}$ disappears. This means that the number of rows of, for instance, matrix $[a]$ is then reduced by one (and also that of $[a l],\{v\}$, etc.). The number of columns of $[a]$ can also be reduced by one if the $i$ th column is multiplied by the known $p_{i}$ and the product added to $\{v\}$.

The same procedure is followed for the remainder of the nodal points of $R_{a}$.

\section{Boundary condition $R_{b}$}

(1) The local value of $q_{n}$ is prescribed.

In that case account has to be taken of (5.23d) alone. As a modification of (5.23d), instead of $q_{n}$ along the whole boundary, $q_{n i}$ in the nodal points $i$ may be prescribed, and the intermediate values can be determined with the help of the interpolation functions $f_{k i}$. If $\left\{q_{n}\right\}$ is the column matrix with elements $q_{n i}$, where

$$
\begin{aligned}
& q_{n i}=0 \quad \text { for } \quad i \notin R_{b}, \\
& q_{n i} \text { is prescribed for } i \in R_{b},
\end{aligned}
$$

then we have for element $k: q_{n}\left(x_{f}, y_{f}\right)=\left\{f_{k}\right\}^{T}\left\{q_{n}\right\}$. Thus (5.23d) becomes:

$$
\{q n\}^{T}=\sum_{k=1}^{m} \int_{R_{k b}}\left\{q_{n}\right\}^{T}\left\{f_{k}\right\}\left\{f_{k}\right\}^{T} \mathrm{~d} R_{k} .
$$

(2) The total value of $q_{n}$ is prescribed for a section of the boundary at which all the nodal-pressure variations are equal.

This implies that the terms of (5.24a) associated with these boundary nodal points may be added, the value $q n_{i}$ being inserted for the sum of the corresponding elements of $\{q n\}$. The number of columns of $[a]$ and $[a l]$ can be reduced by addition of the columns associated with this boundary.

Boundary condition $R_{c}$

Let $i$ and $j$ be two corresponding nodal points on the two adjacent boundaries. We may then write 


$$
\begin{aligned}
& -78- \\
& \delta p_{i}=\delta p_{j} .
\end{aligned}
$$

This means that the coefficients of (5.24a) corresponding to $\delta p_{i}$ and $\delta p_{j}$ may be added and that the corresponding rows in $[a]$ and $[a l]$ may be added, too (and so may the "rows" of $\{v\},\{q s\}$, and $\{q n\}$ ). The number of columns of $[a]$ and $[a l]$ is reduced by one, because of

$$
p_{t}=p_{i},
$$

and the $i$ th and $j$ th columns of $[a]$ and $[a l]$ can be combined to a single column by addition.

\section{Boundary $R_{d}$}

This boundary condition merely affects $(5.23 \mathrm{e})$. In this equation, $\lambda$ can further depend upon the coordinates $x_{f}$ and $y_{f}$. Just as we saw for $q_{n}$ in the case of $R_{b}$, the column matrix $\{\lambda\}$ made up of the nodal-point values $\lambda_{i}$, can be formed with

$$
\begin{aligned}
& \lambda_{i} \equiv 0, \quad \text { for } i \notin R_{d}, \\
& \lambda_{i} \text { is prescribed, for } i \in R_{d},
\end{aligned}
$$

which results in

$$
\lambda\left(x_{f}, y_{f}\right)=\left\{f_{k}\right\}^{T}\{\lambda\}
$$

Thus (5.23e) becomes

$$
[a l]=\sum_{k=1}^{m} \int_{R_{k d}}\left\{f_{k}\right\}\left\{f_{k}\right\}^{T}\{\lambda\}\left\{f_{k}\right\}^{T} \mathrm{~d} R_{k}
$$

If the boundary conditions are applied in the manner just referred to, the remaining pressure variations are independent and we obtain the solution of the pressure field from the set of equations *)

$$
\left[a^{*}\right]\left\{p^{*}\right\}-\left\{v^{*}\right\}+\left\{q s^{*}\right\}+\left\{q n^{*}\right\}+\left[a l^{*}\right]\left\{p^{*}\right\}=0 .
$$

\subsection{The finite-element method based on the "generalised" Reynolds equation}

\subsubsection{The generalised Reynolds equation}

If to a viscous flow of liquid between two surfaces the Reynolds differential equation is applicable and if one of the surfaces is provided with identically equal, parallel and equidistant grooves with a period $l_{g}$ (fig. 5.5), then the pres-

*) The index * has been added to distinguish the symbols used in (5.24b), from the corresponding symbols of (5.24a), where the boundary conditions still had to be applied. 
sure distribution can be imagined as being built up of a smoothed pressure $p_{\text {sm }}$ and a superimposed fluctuating pressure with period $l_{g}$ (fig. 5.6).

As it is just the smoothed pressure that proves to be of importance in the determination of the load capacity we shall, in what follows, derive a "generalised" Reynolds equation for that pressure.

From (5.10) and (5.11) and fig. 5.5 it follows that

$$
\begin{aligned}
& q_{s}=-\left(h+h_{s}\right)^{3} \frac{\partial p}{\partial s}+\frac{1}{2}\left(h+h_{s}\right) u_{s} \\
& q_{r}=-\left(h+h_{s}\right)^{3} \frac{\partial p}{\partial r}+\frac{1}{2}\left(h+h_{s}\right) u_{r} \\
& \frac{\partial q_{r}}{\partial r}+\frac{\partial q_{s}}{\partial s}+\frac{\partial h}{\partial \tau}=0
\end{aligned}
$$

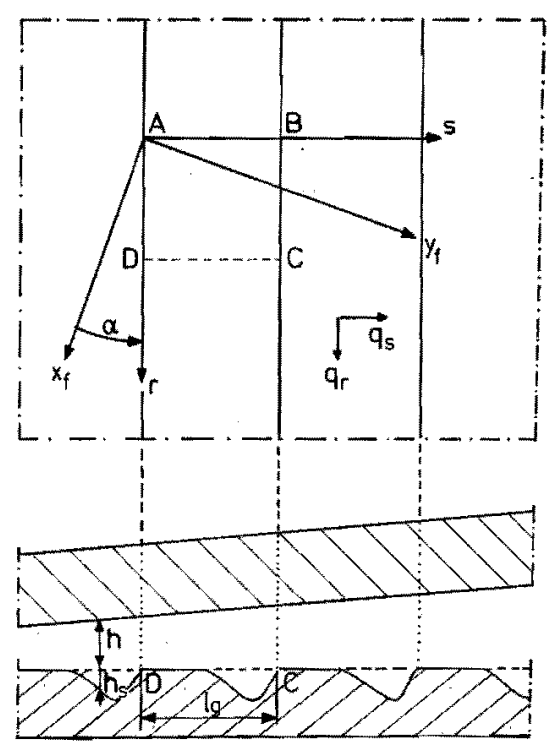

Fig. 5.5. Plan view and cross-section of a grooved bearing section.

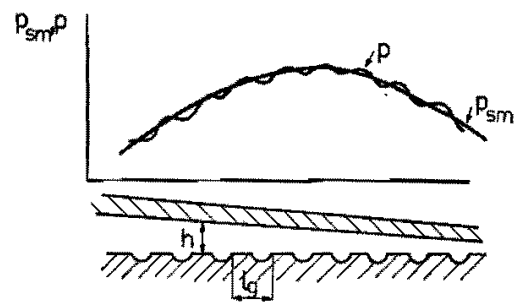

Fig. 5.6. Smoothing of the local pressure $\rho$. 
in which we have

$$
\begin{array}{ll}
q_{r}=q_{r}(r, s, \tau) & \text { flow in } r \text {-direction, } \\
q_{s}=q_{s}(r, s, \tau) & \text { flow in s-direction, } \\
u_{r}=u_{r}(r, s, \tau) & \text { velocity of grooveless surface in } r \text {-direction, } \\
u_{s}=u_{s}(r, s, \tau) & \text { velocity of grooveless surface in } s \text {-direction, } \\
p=p(r, s, \tau) & \text { pressure in film, } \\
h=h(r, s, \tau) & \text { film thickness, } \\
h_{s}=h_{s}(s) & \text { groove depth, } \\
\tau & \text { time, } \\
l_{g} & \text { period of grooves. }
\end{array}
$$

The above-mentioned parameters are dimensionless and are based on the same reference parameters as used in (5.6).

For the special case that

$$
\frac{\partial h}{\partial r}=\frac{\partial h}{\partial s}=\frac{\partial h}{\partial \tau}=0
$$

it can be readily shown that there is a solution of $(5.25)$ and of $(5.26)$, of the following form:

$$
\begin{aligned}
& q_{s}=\text { constant }, \\
& q_{r}=q_{r}(s), \\
& \frac{\partial p}{\partial s}=\text { function }(s), \\
& \frac{\partial p}{\partial r}=\text { constant. }
\end{aligned}
$$

In the calculating of bearings it is often found that the boundary conditions are such that for a small area - for instance of width $l_{g}$ - the relations of $(5.28)$ are approximately satisfied. We now make use of this fact in the choice of the integrands for the integration of (5.25) and (5.26) over an $s$-interval of width $l_{g}$ :

$$
\begin{aligned}
& \frac{1}{l_{g}} \int_{a}^{b} \frac{q_{s} \mathrm{~d} s}{\left(h+h_{s}\right)^{3}}=-\frac{1}{l_{g}} \int_{a}^{b} \frac{\partial p}{\partial s} \mathrm{~d} s+\frac{u_{s}}{2 l_{g}} \int_{a}^{b} \frac{\mathrm{d} s}{\left(h+h_{s}\right)^{2}}, \\
& \frac{1}{l_{g}} \int_{a}^{b} q_{r} \mathrm{~d} s=-\frac{1}{l_{g}} \int_{a}^{b}\left(h+h_{s}\right)^{3} \frac{\partial p}{\partial r} \mathrm{~d} s+\frac{u_{r}}{2 l_{g}} \int_{a}^{b}\left(h+h_{s}\right) \mathrm{d} s,
\end{aligned}
$$




$$
\frac{1}{l_{g}} \int_{a}^{b}\left(\frac{\partial q_{r}}{\partial r}+\frac{\partial q_{s}}{\partial s}+\frac{\partial h}{\partial \tau}\right) \mathrm{d} s=0
$$

in which

$$
a=s-\frac{l_{g}}{2} ; \quad b=s+\frac{l_{g}}{2} .
$$

We now define

the smoothed pressure:

$$
p_{\mathrm{sm}}=\frac{1}{l_{g}} \int_{a}^{b} p \mathrm{~d} s,
$$

the smoothed flow in the $r$-direction:

$$
q_{\mathrm{sm} r}=\frac{1}{l_{g}} \int_{a}^{b} q_{r} \mathrm{~d} s
$$

and:

$$
I_{n}=\frac{1}{l_{g}} \int_{a}^{b}\left(h+h_{s}\right)^{n} \mathrm{~d} s .
$$

This yields:

$$
\begin{gathered}
\frac{1}{l_{g}} \int_{a}^{b} \frac{q_{s} \mathrm{~d} s}{\left(h+h_{s}\right)^{3}}=-\frac{\partial p_{\mathrm{sm}}}{\partial s}+\frac{u_{s}}{2} I_{-2}, \\
q_{\mathrm{sm} \mathrm{r}}=-\frac{1}{l_{g}} \int_{a}^{b}\left(h+h_{s}\right)^{3} \frac{\partial p}{\partial r} \mathrm{~d} s+\frac{u_{r}}{2} I_{+1}, \\
\frac{\partial q_{\mathrm{sm} r}}{\partial r}+\frac{1}{l_{g}} \int_{a}^{b} \frac{\partial q_{s}}{\partial s} \mathrm{~d} s+\frac{1}{l_{g}} \int_{a}^{b} \frac{\partial h}{\partial \tau} \mathrm{d} s=0 .
\end{gathered}
$$

If we approximate the set of equations (5.34) and (5.35) by the following set of equations:

$$
I_{-3} q_{s}=-\frac{\partial p_{\mathrm{sm}}}{\partial s}+\frac{u_{s}}{2} I_{-2},
$$




$$
\begin{gathered}
-82- \\
q_{\mathrm{sm} r}=-I_{+3} \frac{\partial p_{\mathrm{sm}}}{\partial r}+\frac{u_{\mathrm{r}}}{2} I_{+1}, \\
\frac{\partial q_{\mathrm{sm} r}}{\partial r}+\frac{\partial q_{s}}{\partial s}+\frac{\partial h}{\partial \tau}=0,
\end{gathered}
$$

the solution for $q_{s}, q_{\mathrm{sm} r}$ and $p_{\mathrm{sm}}$ obtainable from (5.36) and (5.37) is usable only if substitution in (5.34) and (5.35) gives merely a negligible error. This leads, for example (depending on the relative magnitude of the terms, other conditions can also be imposed), to the following requirements:

$$
\begin{aligned}
& \left|I_{-3} q_{s}-\frac{1}{l_{g}} \int_{a}^{b} \frac{q_{s} \mathrm{~d} s}{\left(h+h_{s}\right)^{3}}\right|=\varepsilon_{1} \ll\left|I_{-3} q_{s}\right|, \\
& \left|I_{+3} \frac{\partial p_{\mathrm{sm}}}{\partial r}-\frac{l}{l_{g}} \int_{a}^{b}\left(h+h_{\mathrm{s}}\right)^{3} \frac{\partial p}{\partial r} \mathrm{~d} s\right|=\varepsilon_{2} \ll\left|I_{+3} \frac{\partial p_{\mathrm{sm}}}{\partial r}\right|, \\
& \left|\frac{\partial q_{s}}{\partial s}-\quad \frac{l}{l_{g}} \int_{a}^{b} \frac{\partial q_{s}}{\partial s} \mathrm{~d} s\right| \quad=\varepsilon_{3} \ll\left|\frac{\partial q_{\mathrm{s}}}{\partial s}\right|, \\
& \left|\frac{\partial h}{\partial \tau}-\quad \frac{1}{l_{g}} \int_{a}^{b} \frac{\partial h}{\partial \tau} \mathrm{d} s\right| \quad=\varepsilon_{4} \ll\left|\frac{\partial h}{\partial \tau}\right| .
\end{aligned}
$$

Here $\varepsilon_{1}$ and $\varepsilon_{2}$ are the errors in respectively (5.36a) and (5.36b), the error in (5.37) being determined by $\varepsilon_{3}$ and $\varepsilon_{4}$.

The quantities $q_{\mathrm{s}}, q_{\mathrm{sm}} r$ and $p_{\mathrm{sm}}$ in (5.38) are approximate values obtained from (5.36) and (5.37). From these, and by the use of (5.25a) and (5.31), we can evaluate the quotient $\partial p / \partial r$ in (5.38b). From (5.25a) follows:

$$
\frac{\partial^{2} p}{\partial s \partial r}=\frac{\partial}{\partial r}\left(\frac{-q_{s}}{\left(h+h_{s}\right)^{3}}+\frac{1}{2} \frac{u_{s}}{\left(h+h_{s}\right)^{2}}\right)
$$

or, as $q_{s}$ is known,

$$
\frac{\partial p}{\partial r}=C(r, \tau)+\text { funct }(r, s, \tau) .
$$

The integration constant $C$ can be calculated from (5.31):

$$
C(r, \tau)=\frac{1}{l_{g}} \int_{a}^{b} C(r, \tau) \mathrm{d} s=\frac{\partial p_{\mathrm{sm}}}{\partial r}-\frac{1}{l_{g_{a}}} \int_{a}^{b} f(r, s, \tau) \mathrm{d} s .
$$


So far the boundary conditions have been left out of consideration. The obvious course is to introduce the boundary conditions of the set of equations (5.36) and of (5.37) by way of the quantities $q_{s}, q_{\mathrm{sm}}$, and $p_{\mathrm{sm}}$. This, however, means that in general the solution obtained no longer satisfies the local boundary conditions. Let us assume, for instance, that at a boundary we have $p=0$. This implies that $p_{\mathrm{sm}}=0$, too; hence:

$$
p=0 \Rightarrow p_{\mathrm{sm}}=0,
$$

but $p_{\mathrm{sm}}=0$ does not imply that $p=0$, for only if $l_{g} \rightarrow 0$ is it true that $p \rightarrow p_{\mathrm{sm}}$, and then $p=0 \Leftrightarrow p_{\mathrm{sm}}=0$.

To determine the pressure variation at the boundary and its effect on the pressure at a sufficiently great distance from the boundary, "the end effect", it is still necessary to solve the equations (5.25) and (5.26) in terms of local quantities (for the determination of the end effect, cf. also Muijderman ${ }^{5-11}$ )).

At this stage we have to answer the question of whether it is permissible to use the smoothed pressure, $p_{\mathrm{sm}}$, instead of the local pressure $p$. We are interested in the load capacity of a bearing, hence in integrals of the type

$$
\int_{r_{1}}^{r_{2}} \int_{s_{1}}^{s_{2}} p \mathrm{~d} r \mathrm{~d} s
$$

Consequently, in addition to the relations (5.38a) to (5.38d), we must impose the following conditions:

$$
\left|\frac{1}{l_{g}} \int_{a}^{b} p \mathrm{~d} s-\frac{1}{l_{g}} \int_{a}^{b} p_{\mathrm{sm}} \mathrm{d} s\right|=\varepsilon_{5} \ll\left|\frac{1}{l_{g}} \int_{a}^{b} p \mathrm{~d} s\right| ;
$$

in other words,

$$
\left|p_{\mathrm{sm}}-\frac{1}{l_{g}} \int_{a}^{b} p_{\mathrm{ssa}} \mathrm{d} s\right|=\varepsilon_{5} \ll\left|p_{\mathrm{sm}}\right|
$$

The relations (5.38) enable us to make an estimate of the accuracy, for very general cases, of a solution of (5.36) and (5.37) and to ascertain whether it is permissible to use the smoothed pressure thus found for calculations of the load capacity.

A particular case where (5.38) is satisfied is obtained if

$$
l_{g} \rightarrow 0 \text {, hence if } b \rightarrow a \text { and } \varepsilon_{k} \rightarrow 0(k=1,2,3,4,5) .
$$

This result has been used by, among others, Vohr and $\operatorname{Pan}^{5-8}$ ) for the derivation of a generalised Reynolds equation, but with respect to (5.38) this is an unnecessarily stringent limitation. 
In vector notation, (5.36) and (5.37) can be written as

$$
\begin{gathered}
\mathbf{q}_{\mathrm{sm}}=-C \nabla p_{\mathrm{sm}}+\frac{1}{2} B \mathbf{u}, \\
\nabla \cdot \mathbf{q}_{\mathrm{sm}}+\frac{\partial h}{\partial \tau}=\mathbf{0} .
\end{gathered}
$$

In the coordinate system $r, s$ (fig. 5.5) the vectors $\mathbf{q}_{\mathrm{sm}}$ and $\mathbf{u}$ can be represented (taking $q_{s}=q_{\mathrm{sm} s}$ ) by

$$
\begin{aligned}
& \mathbf{q}_{\mathrm{sm}}: \quad\left\{q_{\mathrm{sm}}\right\}_{r s}=\left\{\begin{array}{l}
q_{\mathrm{sm}} r \\
q_{\mathrm{sm} s} s
\end{array}\right\}, \\
& \mathbf{u}:\{u\}_{r s}=\left\{\begin{array}{l}
u_{r} \\
u_{s}
\end{array}\right\},
\end{aligned}
$$

and $\nabla, C$ and $B$ by

$$
\begin{aligned}
& \nabla:\{\nabla\}_{r s}=\left\{\begin{array}{c}
\frac{\partial}{\partial r} \\
\frac{\partial}{\partial s}
\end{array}\right\}, \\
& B: \quad[B]_{r s}=\left[\begin{array}{cc}
I_{+1} & 0 \\
0 & \frac{I_{-2}}{I_{-3}}
\end{array}\right], \\
& C: \quad[C]_{r s}=\left[\begin{array}{cc}
I_{+3} & 0 \\
0 & \frac{1}{I_{-3}}
\end{array}\right] .
\end{aligned}
$$

The indices $r s$ are used here to indicate that the $(r, s)$-system applies.

In the $\left(x_{f}, y_{f}\right)$-system (see fig. 5.5) the components of $\mathbf{q}_{\mathrm{sm}}$, $\mathbf{u}$, and $\nabla$ can be obtained by changing $(r, s)$ into $\left(x_{f}, y_{f}\right)$. The components of $B$ and $C$ can be deduced from

$$
\begin{aligned}
& {[B]_{x_{f y_{f}}}=[T][B]_{r s}[T]^{-1},} \\
& {[C]_{x_{y^{y}}}=[T][C]_{r s}[T]^{-1},}
\end{aligned}
$$

with

$$
[T]=\left[\begin{array}{rr}
\cos \alpha & -\sin \alpha \\
\sin \alpha & \cos \alpha
\end{array}\right]
$$


On working this out we obtain:

$$
\begin{aligned}
& B=\left[\begin{array}{ll}
I_{+1} \cos ^{2} \alpha+\frac{I_{-2}}{I_{-3}} \sin ^{2} \alpha & \left(I_{+1}-\frac{I_{-2}}{I_{-3}}\right) \sin \alpha \cos \alpha \\
\left(I_{+1}-\frac{I_{-2}}{I_{-3}}\right) \sin \alpha \cos \alpha & I_{+1} \sin ^{2} \alpha+\frac{I_{-2}}{I_{-3}} \cos ^{2} \alpha
\end{array}\right], \\
& C=\left[\begin{array}{ll}
I_{+3} \cos ^{2} \alpha+\frac{\sin ^{2} \alpha}{I_{-3}} & \left(I_{+3}-\frac{1}{I_{-3}}\right) \sin \alpha \cos \alpha \\
\left(I_{+3}-\frac{1}{I_{-3}}\right) \sin \alpha \cos \alpha & I_{+3} \sin ^{2} \alpha+\frac{\cos ^{2} \alpha}{I_{-3}}
\end{array}\right] .
\end{aligned}
$$

By eliminating $\mathbf{q}_{\mathrm{sm}}$ one finds from (5.42) and (5.43) the generalised Reynolds equation:

$$
\nabla \cdot\left(C \nabla p_{\mathrm{sm}}\right)-\frac{1}{2} \nabla \cdot(B \mathbf{u})-\frac{\partial h}{\partial \tau}=0
$$

5.4.2. Transformation of the generalised Reynolds equation into a variation integral

The derivation is similar to that of the ordinary Reynolds equation given in the preceding section. Instead of (5.13) we obtain:

$$
\delta \Phi=-\int_{G}\left(\nabla \cdot\left(C \nabla p_{\mathrm{sn}}\right)-\frac{1}{2} \nabla \cdot(B \mathbf{u})-\frac{\partial h}{\partial \tau}\right) \delta p_{\mathrm{sm}} \mathrm{d} G=0 .
$$

Given that

$$
\begin{aligned}
\int_{G}\left[\nabla \cdot\left(C \nabla p_{\mathrm{sm}}\right)\right] \delta p_{\mathrm{sm}} \mathrm{d} G & =\int_{G}\left[\nabla \cdot\left(\delta p_{\mathrm{sm}} C \nabla p_{\mathrm{sm}}\right)\right] \mathrm{d} G-\int_{G}\left(C \nabla p_{\mathrm{sm}} \cdot \nabla \delta p_{\mathrm{sm}}\right) \mathrm{d} G \\
& =\int_{R}\left(C \nabla p_{\mathrm{sm}} \cdot \mathbf{n}\right) \delta p_{\mathrm{sm}} \mathrm{d} R-\delta \int_{G}\left(\frac{1}{2} C \nabla p_{\mathrm{sm}} \cdot \nabla p_{\mathrm{sm}}\right) \mathrm{d} G,
\end{aligned}
$$

$$
\begin{aligned}
-\frac{1}{2} \int_{G}[\nabla(B \mathbf{u})] \delta p_{\mathrm{sm}} \mathrm{d} G & =-\frac{1}{2} \int_{G}\left[\nabla \cdot\left(\delta p_{\mathrm{sm}} B \mathbf{u}\right)\right] \mathrm{d} G+\frac{1}{2} \int_{G}\left(B \mathbf{u} \cdot \nabla \delta p_{\mathrm{sm}}\right) \mathrm{d} G \\
& =-\frac{1}{2} \int_{R}(B \mathbf{u} \cdot \mathbf{n}) \delta p_{\mathrm{sm}} \mathrm{d} R+\frac{1}{2} \delta \int_{G}\left(B \mathbf{u} \cdot \nabla p_{\mathrm{sm}}\right) \mathrm{d} G \\
& -\int_{G} \frac{\partial h}{\partial \tau} \delta p_{\mathrm{sm}} \mathrm{d} G=-\delta \int_{G} \frac{\partial h}{\partial \tau} p_{\mathrm{sm}} \mathrm{d} G
\end{aligned}
$$


and

$$
\begin{aligned}
\delta \Phi=0= & -\delta \int_{G}\left(C \nabla p_{\mathrm{sm}} \cdot \nabla p_{\mathrm{ssm}}-\frac{1}{2} B \mathbf{u} \cdot \nabla p_{\mathrm{sm}}+\frac{\partial h}{\partial \tau} p_{\mathrm{sm}}\right) \mathrm{d} G+ \\
& -\int_{\boldsymbol{R}}\left[\left(-C \nabla p_{\mathrm{sm}}+\frac{1}{2} B \mathbf{u}\right) \cdot \mathbf{n}\right] \delta p_{\mathrm{sm}} \mathrm{d} R
\end{aligned}
$$

we can now write, by analogy with (5.22):

$$
\begin{aligned}
\delta \Phi= & -\delta \sum_{k=1}^{m} \int_{G_{k}}\left(\frac{1}{2}\{p\}^{T}\left[r_{k}\right]^{T}[C]\left[r_{k}\right]\{p\}-\frac{1}{2}\{u\}^{T}[B]\left[r_{k}\right]\{p\}+\right. \\
& \left.+\frac{\partial h}{\partial \tau}\left\{f_{k}\right\}^{T}\{p\}\right) \mathrm{d} G_{k}+ \\
& -\delta \sum_{k=1}^{m} \int_{R_{k b}} q_{n}\left\{f_{k}\right\}^{T}\{p\} \mathrm{d} R_{k}+ \\
& -\sum_{k=1}^{m} \int_{R_{k d}} \frac{\lambda}{2}\{p\}^{T}\left\{f_{k}\right\}\left\{f_{k}\right\}^{T}\{p\} \mathrm{d} R_{k},
\end{aligned}
$$

and with$$
\begin{aligned}
& {[a]=\sum_{k=1}^{m} \int_{G_{k}}\left[r_{k}\right]^{T}[C]\left[r_{k}\right] \mathrm{d} G_{k},} \\
& \{v\}^{T}=\sum_{k=1}^{m} \int_{G_{k}}\{u\}^{T}[B]\left[r_{k}\right] \mathrm{d} G_{k},
\end{aligned}
$$$$
\{q s\}^{T}=\sum_{k=1}^{m} \int_{G_{k}} \frac{\partial h}{\partial \tau}\left\{f_{k}\right\}^{T}\{p\} \mathrm{d} G_{k},
$$$$
\{q n\}^{T}=\sum_{k=1}^{m} \int_{R_{k b}} q_{n}\left\{f_{k}\right\}^{T}\{p\} \mathrm{d} R_{k},
$$$$
[a l]=\sum_{k=1}^{m} \int_{R_{k d}} \lambda\left\{f_{k}\right\}\left\{f_{k}\right\}^{T} \mathrm{~d} R_{k},
$$

it follows that

$$
\delta\{p\}^{T}([a]\{p\}-\{v\}+\{q s\}+\{q n\}+[a l]\{p\})=0 .
$$


This equation is equal to eq. (5.24a) and the given boundary conditions can be introduced in the same way.

\subsubsection{The integrals $I_{-3}, I_{-2}, I_{+1}$ and $I_{+3}$ for rectangular and triangular grooves $\left.{ }^{*}\right)$}

Rectangular-groove pattern (fig. 5.7)

We assume the groove pattern to be sufficiently fine, so that

then

$$
\left.\begin{array}{l}
h \approx \text { constant } \\
h+h_{s}=h_{\mathrm{gr}} \approx \text { constant }
\end{array}\right\} \text { for } 0 \leqslant s \leqslant ; l_{g}
$$

$$
\begin{aligned}
& I_{-3}=\frac{1}{l_{g}}\left(\int_{0}^{\left[\gamma /(1+\gamma] l_{g}\right.} \frac{\mathrm{d} s}{h^{3}}+\int_{[\gamma /(1+\gamma)]]_{g}}^{l_{g}} \frac{\mathrm{d} s}{h_{\mathrm{gr}}{ }^{3}}\right)=\frac{1}{1+\gamma}\left(\frac{\gamma}{h^{2}}+\frac{1}{h_{\mathrm{gr}}{ }^{3}}\right), \\
& I_{-2}=\frac{1}{1+\gamma}\left(\frac{\gamma}{h^{2}}+\frac{1}{h_{\mathrm{gr}}{ }^{2}}\right), \\
& I_{+1}=\frac{1}{1+\gamma}\left(\gamma h+h_{\mathrm{gr}}\right), \\
& I_{+3}=\frac{1}{1+\gamma}\left(\gamma h^{3}+h_{\mathrm{gr}}{ }^{3}\right) .
\end{aligned}
$$

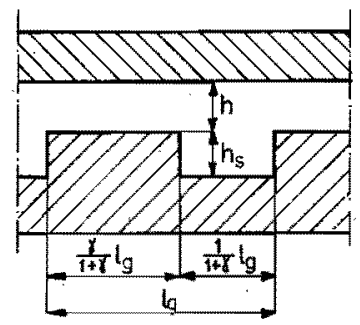

Fig. 5.7. Rectangular groove shape

Triangular groove pattern (fig. 5.8)

We assume that

$$
h \approx \text { constant for } 0 \leqslant s \leqslant l_{g} .
$$

It can easily be shown that the outcome of the integrals is independent of the groove shape and is governed solely by the width and depth of the groove.

Hence, for the calculation, we start from a groove with the shape of a rightangled triangle (indicated in fig. 5.8 by a dotted line).

*) Reference should be made also to Ir J. Bootsma, "Spiral groove bearings with arbitrary groove shapes", Philips Res. Lab. Techn. Note No. 96/69. 


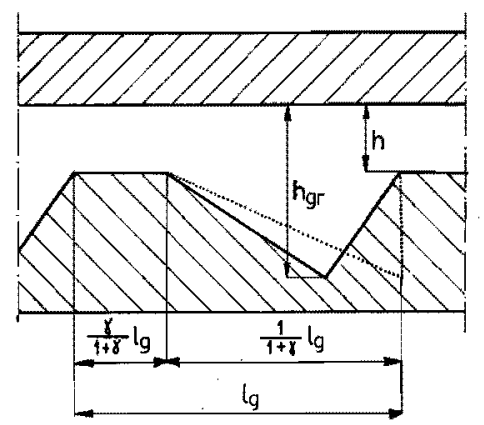

Fig. 5.8. Triangular groove shape.

We have

$$
\begin{aligned}
& I_{-3}=\frac{1}{1+\gamma}\left(\gamma h^{-3}-\frac{h_{\mathrm{gr}}^{2}-h^{2}}{2\left(h_{\mathrm{gr}}-h\right)}\right), \\
& I_{-2}=\frac{1}{1+\gamma}\left(\gamma h^{-2}-\frac{h_{\mathrm{gr}}{ }^{-1}-h^{-1}}{h_{\mathrm{gr}}-h}\right), \\
& I_{+1}=\frac{1}{1+\gamma}\left(\gamma h+\frac{h_{\mathrm{gr}}{ }^{2}-h^{2}}{2\left(h_{\mathrm{gr}}-h\right)}\right) \\
& I_{+3}=\frac{1}{1+\gamma}\left(\gamma h^{3}+\frac{h_{\mathrm{gr}}{ }^{4}-h^{4}}{4\left(h_{\mathrm{gr}}-h\right)}\right) .
\end{aligned}
$$

\subsection{Example of an element: the triangular element}

A very simple element is a triangle with the angular points as nodal points, the interpolation functions being linear. Let $\mathbf{x}_{f i}, \mathbf{x}_{f j}, \mathbf{x}_{f^{\prime} k}$ be the coordinates of the nodal points (fig. 5.9). By means of interpolation functions the pressure (we shall not distinguish between $p$ and $p_{\mathrm{sm}}$ and just write $p$ ) in element $e$ can then be described by

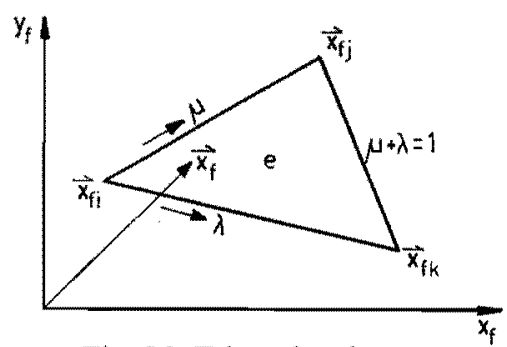

Fig. 5.9. Triangular element.

$$
\begin{gathered}
p\left(\mathbf{x}_{f}\right)=f_{e i} p\left(\mathbf{x}_{f i}\right)+f_{e j} p\left(\mathbf{x}_{f j}\right)+f_{e k} p\left(\mathbf{x}_{f k}\right), \\
\nabla p=p\left(\mathbf{x}_{f i}\right) \nabla f_{e i}+p\left(\mathbf{x}_{f j}\right) \nabla f_{e j}+p\left(\mathbf{x}_{f k}\right) \nabla f_{e k} .
\end{gathered}
$$

Introducing the new coordinates $\lambda$ and $\mu$, we can rewrite $\mathbf{x}_{f}$ (cf. fig. 5.9) as 


$$
\mathbf{x}_{f}=\mathbf{x}_{f i}+\lambda\left(\mathbf{x}_{f k}-\mathbf{x}_{f i}\right)+\mu\left(\mathbf{x}_{f j}-\mathbf{x}_{f i}\right)
$$

It follows that

$$
\begin{aligned}
f_{e i} & =1-\mu-\lambda, \\
f_{e j} & =\mu \\
f_{e k} & =\lambda \\
\left\{\nabla f_{e h}\right\} & =\left\{\begin{array}{ll}
\frac{\partial f_{e h}}{\partial \mu} \frac{\partial \mu}{\partial x_{f}}+\frac{\partial f_{e h}}{\partial \lambda} \frac{\partial \lambda}{\partial x_{f}} \\
\frac{\partial f_{e h}}{\partial \mu} \frac{\partial \mu}{\partial y_{f}}+\frac{\partial f_{e h}}{\partial \lambda} \frac{\partial \lambda}{\partial y_{f}}
\end{array}\right\}
\end{aligned}
$$

From (5.55):$$
\frac{\partial \lambda}{\partial x_{f}}=\frac{y_{f_{j}}-y_{f i}}{a_{p}}
$$$$
\frac{\partial \mu}{\partial x_{f}}=\frac{y_{f i}-y_{f k}}{a_{p}},
$$$$
\frac{\partial \lambda}{\partial y_{f}}=\frac{x_{f i}-x_{f j}}{a_{p}} .
$$$$
\frac{\partial \mu}{\partial y_{f}}=\frac{x_{f k}-x_{f i}}{a_{p}}
$$

with

$$
a_{p}=\left|\begin{array}{ll}
x_{f k}-x_{f i} & x_{f j}-x_{f i} \\
y_{f k}-y_{f i} & y_{f j}-y_{f i}
\end{array}\right|=2 \times \text { area of the triangle. }
$$

From (5.57) and (5.58):

$$
\begin{aligned}
& \left\{\nabla f_{e i}\right\}=\frac{1}{a_{p}}\left\{\begin{array}{l}
y_{f k}-y_{f j} \\
x_{f j}-x_{f k}
\end{array}\right\} \\
& \left\{\nabla f_{e j}\right\}=\frac{1}{a_{p}}\left\{\begin{array}{l}
y_{f i}-y_{f k} \\
x_{f k}-x_{f i}
\end{array}\right\}, \\
& \left\{\nabla f_{e k}\right\}=\frac{1}{a_{p}}\left\{\begin{array}{l}
y_{f j}-y_{f i} \\
x_{f i}-x_{f j}
\end{array}\right\} ;
\end{aligned}
$$

$i$ th column $\quad j$ th column $k$ th column $\left[r_{k}\right]=\frac{1}{a_{D}}\left[\begin{array}{l}0,0, \ldots, 0,\left(y_{f k}-y_{f j}\right) \ldots\left(y_{f i}-y_{f k}\right), \ldots\left(y_{f j}-y_{f i}\right)_{,} \ldots 0, \ldots \\ 0,0, \ldots, 0,\left(x_{f j}-x_{f k}\right)_{1} \ldots\left(x_{f k}-x_{f i}\right), \ldots\left(x_{f i}-x_{f j}\right)_{,} \ldots 0, \ldots\end{array}\right]$. 
To calculate expressions (5.23) and (5.50) it may be necessary to determine integrals of the type

$$
\begin{aligned}
& I_{G i j}^{(n m)}=\int_{G_{k}}\left(f_{e i}\right)^{n}\left(f_{e j}\right)^{m} \mathrm{~d} G_{k} \quad i=1,2,3,
\end{aligned}
$$

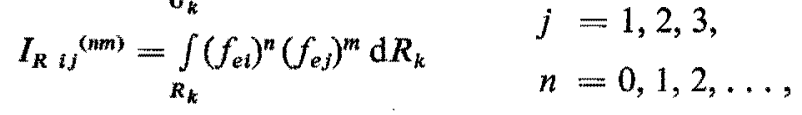

$$
\begin{aligned}
& m=0,1,2, \ldots \text {. }
\end{aligned}
$$

The solution of these integrals is a simple matter provided that we use the coordinates $\lambda$ and $\mu$. For example:

$$
\int_{G_{k}}\left(f_{e i}\right)^{n}\left(f_{e j}\right)^{m} \mathrm{~d} G_{k}=\iint\left(f_{e i}\right)^{n}\left(f_{e j}\right)^{m} \mathrm{~d} x_{f} \mathrm{~d} y_{f}=\iint\left(f_{e i}\right)^{n}\left(f_{e j}\right)^{m} \operatorname{det} \mathrm{d} \lambda \mathrm{d} u,
$$

in which

$$
\operatorname{det}=\left|\begin{array}{ll}
\frac{\partial x_{f}}{\partial \lambda} & \frac{\partial x_{f}}{\partial \mu} \\
\frac{\partial y_{f}}{\partial \lambda} & \frac{\partial y_{f}}{\partial \mu}
\end{array}\right|=a_{p}
$$

\section{REFERENCES}

3-1) J. F. Besseling, The complete analogy between the matrix equations and the field equations of structural analysis, International Symposium on Analog and Digital Techniques Applied to Aeronautics, Liège (1963).

5-2) O. C. Zienkiewicz and Y.K. Cheung, The finite element method in structural and continuum mechanics, McGraw-Hill Publ. Co. Ltd, London, 1970.

${ }^{5-3}$ ) M. M. Reddi, Finite element solution of the incompressible lubrication problem, Trans. ASME, J. Lub. Techn. 91, 524-533, 1969.

5-4) M. M. Reddi and T. Y. Chu, Finite element solution of the steady-state compressible lubrication problem, ASLE-ASME Lub. Conference Houston, Texas (Oct. 1969).

5-5) J. H. Argyris and D. W. Scharpf, The incompressible lubrication problem, The Aeronautical Journal of the Royal Aeronautical Society 73, 1044, 1969.

5-6) G. Vogelpohl, Beiträge zur Kenntnis der Gleitlagerreibung, VDI-Forschungsheft 386, edition B, vol. 8, Oct. 1937.

5-7) G. G. Hirs, The load capacity and stability characteristics of hydrodynamic grooved journal bearings, Trans. ASLE 8, 296-305, 1965.

5-8) J. H. Vohr and C. H. T. Pan, On the spiral-grooved, self-acting gas bearing, MTI report no. 63TR52, Jan. 1964.

${ }^{5-9}$ ) A. Cameron, Principles of lubrication, Longmans Green and Co. Ltd, London, 1966.

5-10) O. Pinkus and B. Sternlicht, Theory of hydrodynamic lubrication, McGrawHill Book Co., Inc., New York, 1961.

5-11) E. A. Muijderman, Spiral-groove bearings, Philips Res. Repts Suppl. 1964, No. 2.

5-12) R. Courant and D. Hilbert, Methods of mathematical physics, Interscience Publishers, 1966. 


\section{CAVitation}

\subsection{Introduction}

If the pressure in the lubricant film of a liquid bearing falls to a certain value, which lies between the vapour pressure of the lubricant and the partial pressure of the dissolved gases, then a further drop in pressure is prevented by the breaking of the coherence of the film, due to "boiling" of the lubricant or escaping of dissolved gases, or both; this phenomenon is called "cavitation". For the time being we shall confine ourselves to cavitation in a steadily loaded bearing.

The flow in the region of cavitation does not obey the Reynolds equation. Figure 6.1 shows this region of cavitation in the lubricant film of a journal bearing. Sometimes part of the boundary of the region of cavitation is fixed due to the presence of a lubricant groove (e.g. Sassenfeld and Walther ${ }^{6-4}$ )), but the position of the boundary is often governed exclusively by both the flow

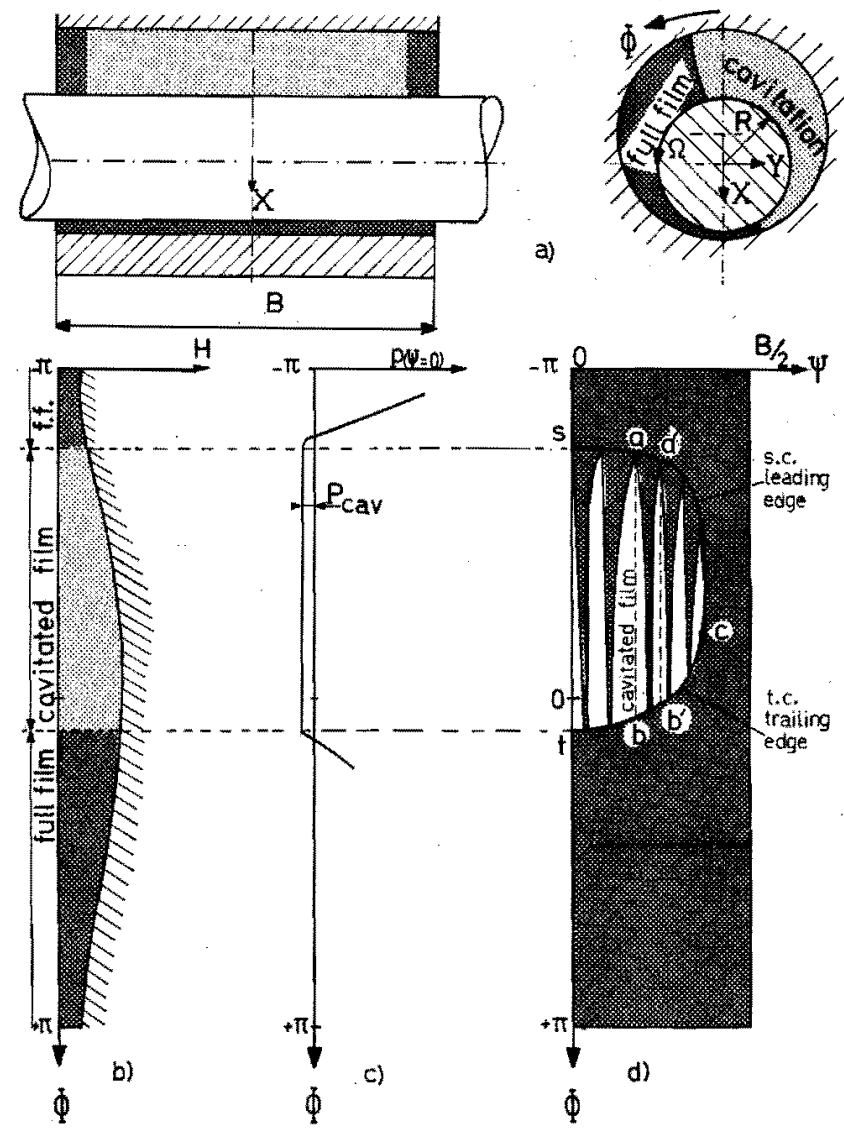

Fig. 6.1. Cavitation, pressure profile and film thickness in a plain journal bearing. 
in the full-film region and that in the cavitation region. The determination of this boundary between two regions with different flow equations complicates the calculation of a cavitating bearing very much. In the literature this problem is usually avoided by specifying such conditions for the cavitation region that the calculation can be made fairly straightforwardly and the situation in the cavitation region is then approximated ${ }^{6-3 / 6}$ ). But these cavitation conditions, which can be handled easily enough in the calculation, frequently describe the flow in the bearing in a physically incorrect manner. By physically correct conditions we should mean a description of the flow in the cavitation region in such a way as to satisfy at least the continuity law throughout the bearing. It is precisely the disregarding of the continuity law that in some cases can give rise to substantial errors in the calculation. That is the reason why we put this law first and foremost as a cavitation condition.

Physically correct cavitation conditions have been established by Jakobson and Floberg ${ }^{6-1}$ ) (and referred to also by Horsnell ${ }^{6-2}$ ). The author is familiar with only one paper ${ }^{6-2}$ ) in which an attempt is made to use the Jakobson and Floberg ( $\mathrm{J} \& \mathrm{~F}$ ) condition in the calculation, but in a later article ${ }^{6-5}$ ) the workers just referred to have decided in favour of approximate cavitation conditions, among other reasons on account of the time-consuming computer calculations. In all these papers it is pointed out that the choice of the cavitation condition does slightly affect the load capacity, maximum pressure, and attitude angle. This is, indeed, often the case, but it does occasionally happen that the deviations become impermissibly great. In what follows it will be shown that, when the J\& F condition is applied to, for example, a smooth journal bearing with circumferential oil grooves, the load capacity wil be a fraction of that following from the - frequently used - data published by Sassenfeld and Walther. It may be reassuring for the users of helically grooved journal bearings, which are frequently fed from just the circumferential grooves, that these bearings, unlike smooth bearings, are far less sensitive to the supply pressure of the lubricant. In general it may be said that the application of an approximate cavitation condition does not yield the true flow balance of a bearing and will cause the largest deviations in those bearings that are sensitive to this effect.

In the following we shall describe a method, called the dummy-flow method, which greatly facilitates the application of the J \& F condition in the computation of a bearing and which consumes little time. Thus the use of approximate cavitation conditions and the associated uncertainty as to the results can be avoided.

\subsection{Cavitation conditions}

\subsubsection{Conditions for smooth journal bearings}

For the sake of clearness we shall first describe the $\mathrm{J} \& \mathrm{~F}$ condition (condi- 
tion 4) and subsequently the conditions 1,2 and 3. In the J\&F condition a flow pattern is proposed, for the cavitation region, which guarantees continuity of the flow everywhere in the bearing and seems to approximate very closely the real flow in a steadily loaded smooth journal bearing ${ }^{6-7,8}$ ).

Condition 4, Jakobson and Floberg conditions

The flow pattern which is in conformity with the J \&F condition is as follows.

From (5.10) it follows that the flow in the full film of a smooth bearing is given by

$$
\mathbf{q}=-h^{3} \nabla p+\frac{1}{2} h \mathbf{u} .
$$

At the leading edge of the cavitation region the pressure in the full film has dropped to a level where cavitation sets in; this means that the component of $\nabla p$ along $\mathbf{u}$ is negative or zero, and

$$
|\mathbf{q}| \geqslant\left|\frac{1}{2} h \mathbf{u}\right| \text {. }
$$

In the cavitation region it is true that

$$
|\mathbf{q}| \leqslant\left|\frac{1}{2} h \mathbf{u}\right| \text {. }
$$

Assuming that $h$ is a continuous function of the film coordinates, (6.2) and (6.3) imply that at the leading edge $\nabla p=0$, and the conditions for the leading edge become:

$$
\nabla p=0 \quad \text { and } \quad p=p_{\text {cav }} .
$$

At the trailing edge the pressure begins to rise again and the relation (6.3) holds for both the cavitation region and the full film. Now the full film may start with $\nabla p>0$, and this is determined by the continuity of flow: if we assume that for smooth surfaces the flow in the cavitation region is parallel to $\mathbf{u}$, then the amount of lubricant entering the cavitation region via aa' (fig. 6.1d) should be equal to the amount leaving across $\mathrm{bb}^{\prime}$. Experiments ${ }^{6-7,8}$ ) support this model of the flow.

Let us next describe the approximate cavitation conditions.

\section{Condition 1}

The pressure distribution in the bearing is calculated as though no cavitation occurred, and hence as though the Reynolds equation did apply in the cavitation region. Next, by putting $p=p_{\text {cav }}$ if it is found that $p<p_{\text {cav }}$, the cavitation region is approximated.

The handling of condition 1 is very simple. However, the pressure gradient at the leading edge (saa'c in fig. 6.1d) is discontinuous, and hence the flow is discontinuous as well. Besides, the flow entering the full film at $b b^{\prime}$ will in general be unequal to the flow crossing at aa'. 
Condition 2

For the leading edge saa'c of the cavitation region we have

$$
\nabla p=0 \quad \text { and } \quad p=p_{\text {cav }}
$$

As this is equivalent to (6.4), the flow at the leading edge will be continuous. Furthermore the trailing edge is fixed at $\Phi=2 \pi$, i.e. at the location of the widest film gap. In general the flow at the trailing edge will then be discontinuous. If an axial lubricant groove is present at $\Phi=2 \pi$, which compensates discontinuities in the flow, then condition 2 is physically correct. This situation has been taken as the starting point in the calculations by Sassenfeld and Walther ${ }^{6-4}$ ).

Condition 2 can readily be used if the field of pressure in the bearing is calculated by an iteration method (cf. ref. 6-5). Then the usual method is to put $p=p_{\text {cav }}$ if, in the iteration proces of nodal pressures, it is found that $p<p_{\text {cav }}$.

\section{Condition 3}

For the leading edge as well as for the trailing edge it is assumed that

$$
\nabla p=0 \quad \text { and } \quad p=p_{\text {cav }}
$$

The liquid flow at the leading edge is continuous. However, the flow leaving the cavitation region via the trailing edge will generally be greater than the flow entering this region via the leading edge. This cavitation can easily be applied in an iteration method of calculation, as also was condition 2.

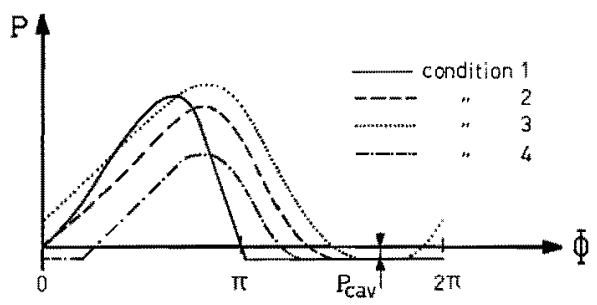

Fig. 6.2. Typical shapes of pressure profiles in the middle of the bearing, for the different cavitation conditions.

The typical shapes of pressure profiles for the different cavitation conditions are displayed in fig. 6.2. Here we have assumed that the reference pressure at either end of the bearing $(\Psi= \pm B / 2)$ is equal to zero.

\subsubsection{Modification of the Jakobson and Floberg conditions for grooved bearings}

For smooth surfaces the $J \& F$ conditions are supported by experiments ${ }^{6-7,8}$ ) which show that the flow frequently crosses the cavitation region of a steadily 
loaded bearing in ribbon-shaped flow paths (streamers) parallel to $\mathbf{u}$. If we assume that, in the streamers, the liquid makes contact with both surfaces and $\nabla p=0$, then (6.1) also forecasts a parallel flow:

$$
\mathbf{q}=\frac{1}{2} h \mathbf{u} \quad \text { (in a streamer). }
$$

Our experiments with grooved surfaces show that in this case the flow is not entirely parallel. This makes a modification of the $J \& F$ conditions for grooved surfaces necessary and we shall base the modification on the equation, corresponding to (6.1), for grooved surfaces:

$$
\mathbf{q}_{\mathrm{sm}}=-C \nabla p_{\mathrm{sm}}+\frac{1}{2} B \mathbf{u} .
$$

Assuming that $\nabla p=0,(6.6)$ reduces to

$$
\mathbf{q}_{\mathrm{sm}}=\frac{1}{2} B \mathbf{u}
$$

and the angle $\alpha_{c}$ between the flow vector, $\mathbf{q}_{\mathrm{sm}}$, and $\mathbf{u}$ (fig. 6.3) becomes

$$
\alpha_{c}=\arccos \left(\frac{B \mathbf{u} \cdot \mathbf{u}}{|B \mathbf{u}| \times|\mathbf{u}|}\right) .
$$

In the modified $J \& F$ condition it is therefore assumed that the flow in the cavitation region makes an angle $\alpha_{c}$ with the direction of $\mathbf{u}$ !

Note. Using (6.8) for smooth surfaces we find $\alpha_{c}=0$, as was to be expected.

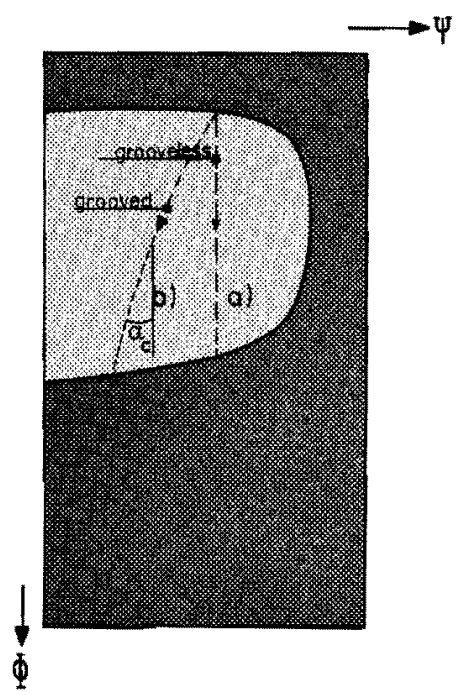

Fig. 6.3. The characteristic flow path in the cavitated film of a plain cylindrical bearing (a) and a helically grooved cylindrical bearing (b). 


\subsection{The dummy-flow method}

We shall now discuss the manner of including the modified J \& F condition in the calculation program.

Assuming that there is no cavitation in the lubricant film the set of equations (5.24b) can be generated. Boundary condition $\boldsymbol{R}_{c}$ will be ignored for this case, so that we obtain:

$$
\left[a^{*}\right]\left\{p^{*}\right\}-\left\{v^{*}\right\}+\left\{q s^{*}\right\}+\left\{q n^{*}\right\}=0 .
$$

By means of (6.9) the unknown nodal pressures may be determined for the case that cavitation is suppressed. The $i$ th equation, corresponding to the $i$ th unknown pressure, may be written as follows $\left(p_{j}^{*}=p_{j}\right)$ :

$$
\sum_{j=1}^{n^{*}} a_{i j}{ }^{*} p_{j}-v_{i}^{*}+q s_{i}^{*}+q n_{i}^{*}=0 .
$$

Equation (6.10) states that the net outflow at nodal point $i$ should be zero. Introducing the symbol $q_{l}$ for the net outflow:

$$
q_{i}=\sum_{j=1}^{n^{*}} a_{l j}^{*} p_{j}-v_{i}^{*}+q s_{i}^{*}+q n_{i}^{*}
$$

and equation (6.10) can be written as

$$
q_{i}=0 .
$$

Let us now consider the same lubricant film, but with cavitation. In the cavitation region the equation of Reynolds does not normally apply; by means of an artifice, however, we can make the Reynolds equation hold also in this region.

For this purpose we make the nodal points leaky, as it were, in the cavitation region, and we keep the pressure in this region equal to $p_{\text {cav }}$ by supplying or drawing off a sufficient quantity of liquid at the location of the nodal points. In this case $q_{i} \neq 0$, and the flow that has to be supplied to the nodal point will be called the dummy flow (fig. 6.4).

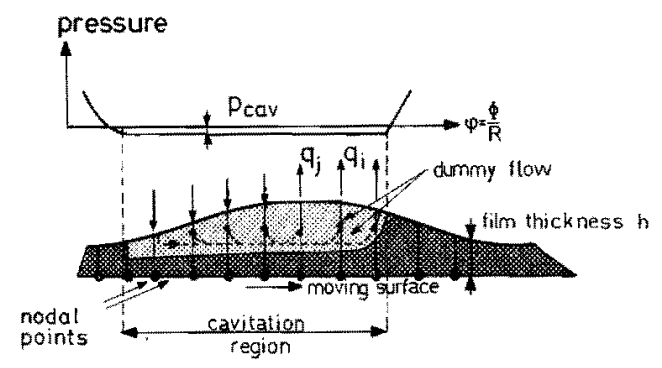

Fig. 6.4. Dummy flow in characteristic flow path of a cavitated film. 
The film diverges in the vicinity of the leading edge and the dummy flow will be positive (fig. 6.4). Near the trailing edge the dummy flows are negative. The dummy flows, together with the main flow, will move along a characteristic flow path (fig. 6.3) to the trailing edge, which is where the sum of the dummy flows along the flow path equals zero.

In case of cavitation an iterative solution of $(6.10)$ is required. In the fullfilm region a new approximation of the pressure in the nodal point $i$ is found from the surrounding nodal-point pressures:

$$
p_{i}=\frac{1}{a_{i i}{ }^{*}}\left(v_{i}^{*}-q s_{i}^{*}-q n_{i}^{*}-\sum_{\substack{j=1 \\ j \neq i}}^{n^{*}} a_{i j}{ }^{*} p_{j}\right)
$$

If it is found that $p<p_{\text {cav }}$, then we are obviously concerned with a nodal point in the cavitation region and we put $p_{i}=p_{\text {cav }}$. With the help of (6.11) the dummy flow for this nodal point is subsequently calculated.

Calculating "downstream" in the cavitation region we again find, near the trailing edge :

However, if

$$
p_{i}>p_{\text {cay }}
$$

$$
\Sigma \text { dummy flows }>0
$$

is applicable to a characteristic flow path, then the generation of positive pressures, and hence that of the trailing edge, should be suppressed and we can put once more $p_{i}=p_{\text {cav }}$ and calculate the dummy flow of $i$. Thus in a specific flow path, the trailing edge cannot begin until the dummy flows just cancel out.

Note. If it is possible for the modified J \& F method to be applied in the calculation program, then use can again be made of the conditions 1, 2 and 3.

For condition 1 this is trivial.

Condition 3 is obtained if the generation of the trailing edge is not suppressed as long as (6.14) is still true.

With condition 2 the trailing edge is fixed and can thus be processed as the "natural" edge.

From the foregoing it follows that in our calculation we have to follow the dummy flow in the cavitation region on its road along a flow path and that we may again accept positive pressures, hence allow the full film to start, only when the dummy flow is fully absorbed again.

If, in a smooth bearing, we adopt a rectangular distribution of nodal points, in which, for instance, the columns of nodal points are parallel to the vector $\mathbf{u}$, then these nodal-point columns will be capable of functioning as characteristic flow paths (since $\alpha_{c}=0$ ) and it will be possible during the calculation to keep 
a record of the total dummy flow per column of nodal points. With a grooved surface, on the other hand, the flow path will then make an angle $\alpha_{c}$ with the columns. As an approximation the total dummy flow, $q_{d}$, can then be supplied to the next row of nodal points, according to the scheme shown in fig. 6.5 .

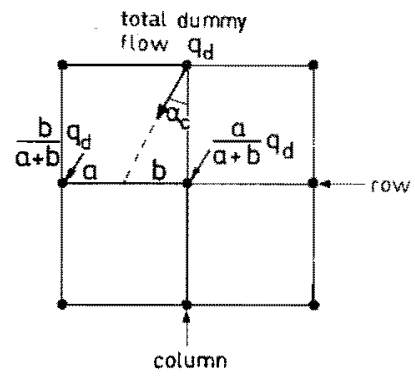

Fig. 6.5. Distribution of total dummy flow $q_{d}$, over the next row of nodal points.

\subsection{Examples}

\subsubsection{Effect of the parameter $p_{\mathrm{cav}}$ on the load capacity of a smooth journal bear-} ing with circumferential feeding

In this section we shall consider an example of a bearing which is highly sensitive to the cavitation condition used. The load capacity of this type of bearing may be largely overestimated if the J\& F condition is not used. The conclusion that can be drawn from this example is that the use of the $\mathrm{J} \& \mathrm{~F}$ condition is at all times preferable, particularly because, with the dummy-flow method, this takes but little extra computing time.

The bearing in question is sketched in fig. 6.6. This smooth journal bearing rotates, as it were, in a medium of lubricant with a (reference) pressure equal to zero. This pressure governs the boundary conditions at either end of the bearing. If the pressure anywhere in the bearing falls below the parameter $p_{\text {cav }}$, then cavitation will occur. The value of this parameter, which is determined by the vapour pressure, the partial pressure of the dissolved gases, the temperature, etc., must be given in order to make the calculation of the bearing possible. This parameter can be calculated from the bearing data, for example.
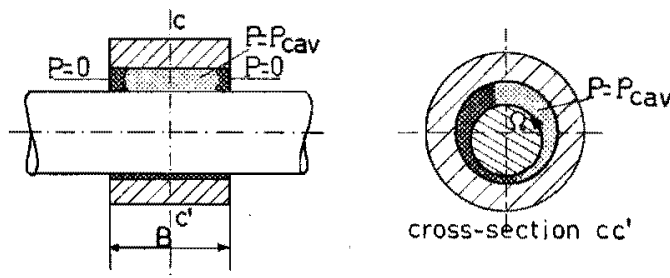

Fig. 6.6. Cavitation in a smooth cylindrical bearing with circumferential feeding. 
Let us assume that a bearing cavitates at a pressure of 1 bar under the ambient pressure,

With

$$
P_{\text {cav }}=-1 \text { bar }=-10^{5} \mathrm{~N} / \mathrm{m}^{2} \text {. }
$$

$$
\eta=0.08 \mathrm{Ns} / \mathrm{m}^{2}, \quad \Omega=100 \mathrm{rad} / \mathrm{s}, \quad R / \Delta R=10^{3},
$$

$p_{\text {cav }}$ becomes:

$$
p_{\text {cav }}=\frac{P_{\text {cav }}}{P_{0}}=\frac{P_{\text {cav }}}{12 \eta \Omega}\left(\frac{\Delta R}{R}\right)^{2}=-10^{-3} .
$$

Figure 6.7 is a plot of the load capacity, calculated with the finite-element method, together with the $J$ \& $F$ cavitation condition (see also chapters 5 and 7 ), of the bearing given in fig. 6.6 as a function of the bearing eccentricity, $e_{x}$, for different values of the parameter $p_{\text {cav }}$. Furthermore the load capacity according to Sassenfeld and Walther ${ }^{6-4}$ ), which is often taken as the basis for the calculation of the load capacity of such a bearing, is drawn in this figure (ALG).

Figure 6.7 shows that for small values of $p_{\text {cav }}$ the load capacity can be a fraction of that calculated by Sassenfeld and Walther ( $\&$ W).

The results do agree with the $\mathrm{S} \& \mathrm{~W}$ data only if $\left|p_{\text {cav }}\right| \approx 0.01$. At high values of $\left|p_{\text {cav }}\right|$, i.e. $\left|p_{\text {cav }}\right|=0 \cdot 1$, the load capacity exceeds that of $\mathrm{S} \& \mathrm{~W}$ because cavitation is suppressed.

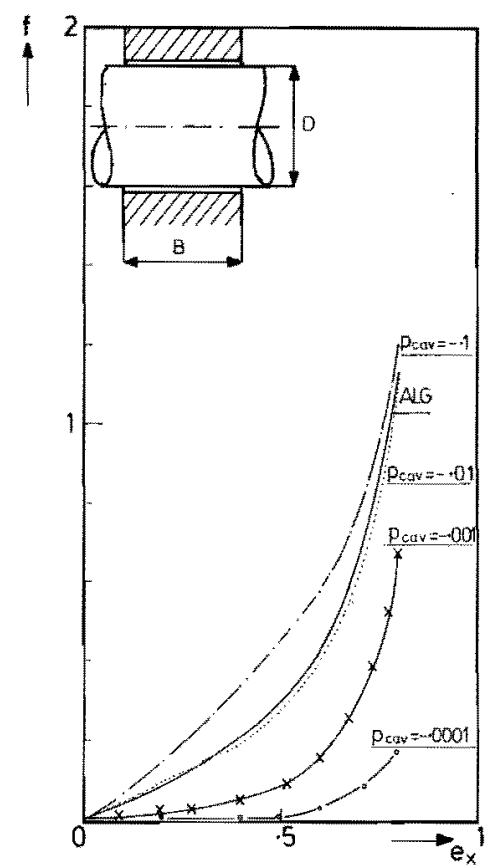

Fig. 6.7. The load capacity of a smooth cylindrical bearing with circumferential feeding $\left(B / D=1, e_{y}=0\right)$. 
Figure 6.8 gives the pressure in the various nodal points of a bearing half for $e_{x}=0.8$ and different values of $p_{\text {cav }}$. The nodal points where the film cavitates are marked by the symbols vvvvvvvv.

The nodal-point coordinates are defined in fig. 6.1. It is clearly seen that the cavitation region increases with decreasing $\left|p_{\text {cav }}\right|$ : the bearing looses, as it were, its lubricant. This can also be understood physically, if it is realised that it is precisely the pressure difference $\left(0-p_{\text {cav }}\right)$ which provides for the filling of the bearing at its edge. It will become more difficult to compensate the leakage on the high-pressure side as the pressure difference $\left(0-p_{\text {cav }}\right)$ decreases.

If $p_{\text {cav }}=0$, the filling mechanism ceases to function, so that the load capacity is found to fall to zero.

Note. An ALG bearing (see chapter 7) with a lubricant groove at the location of the widest film gap is filled in quite another way. Here, independently of $p_{\text {cav }}$, the full film starts at all times at the widest film gap, and it is practically impossible for the bearing to "loose its lubricant". In this case $p_{\text {car }}$ has far less influence. Sassenfeld and Walther used for their calculations $p_{\text {cav }}=0$, a value at which, in the bearing just described, a complete loss of load capacity does in fact result.

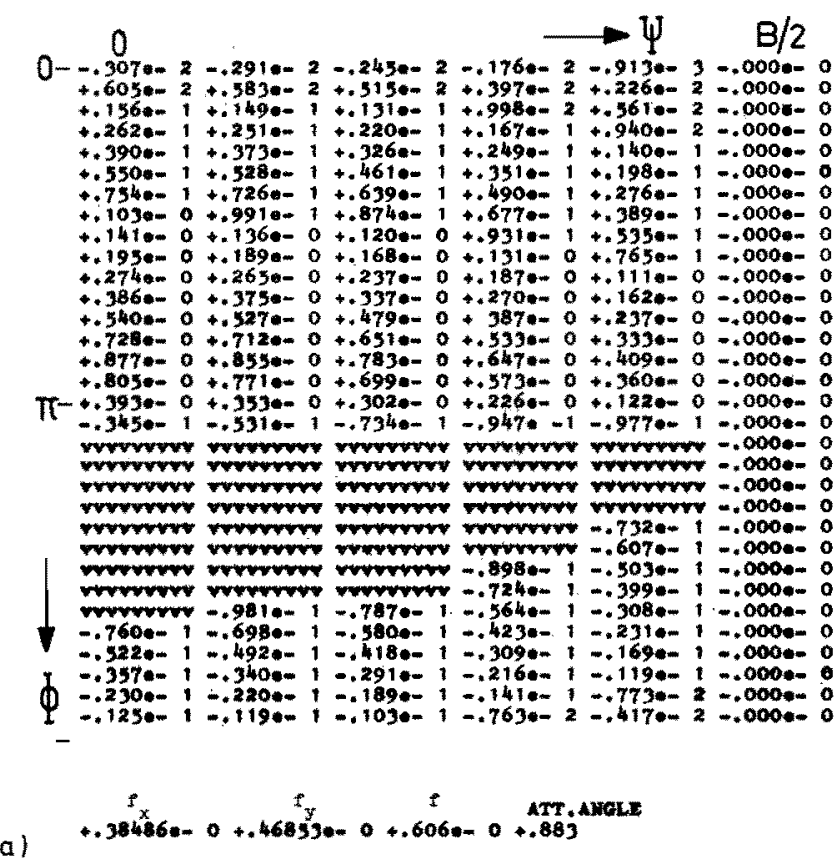

Fig. 6.8a. The pressure in the various nodal points of a bearing half; $e_{x}=0.8, e_{y}=0$, $p_{\text {eav }}=-0 \cdot 10$. 


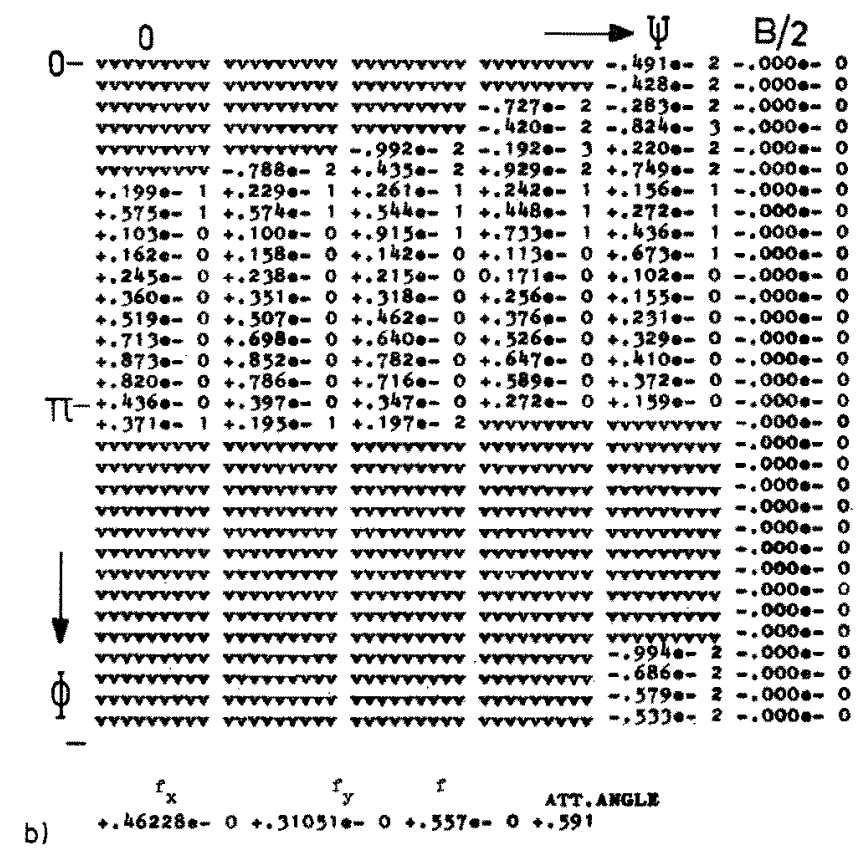

Fig. 6.8b. The pressure in the various nodal points of a bearing half; $e_{x}=0.8, e_{y}=0$, $p_{\mathrm{cav}}=-0.01$.

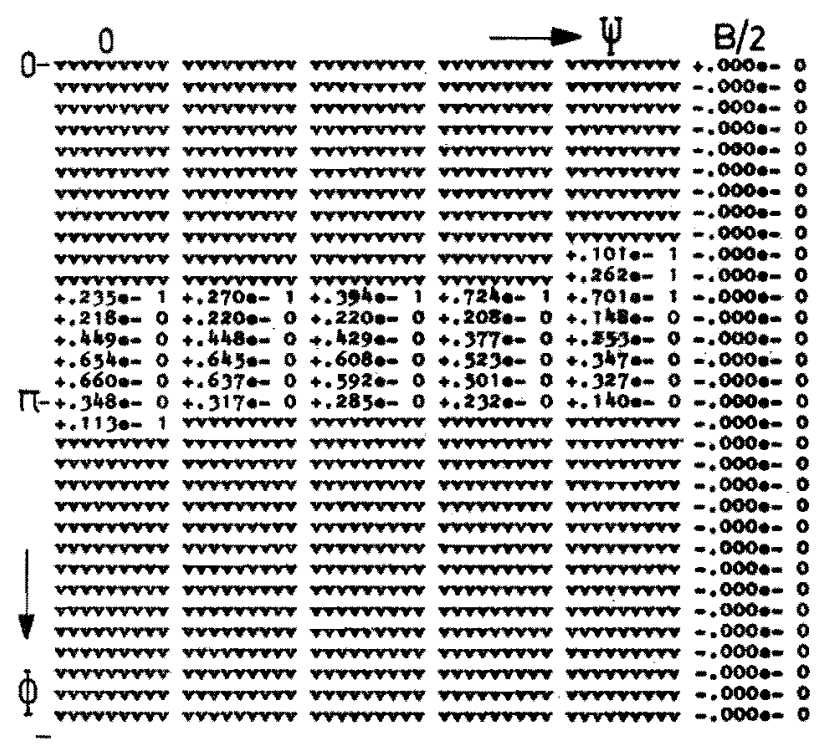

\footnotetext{
c) $+.314100=0+.12709=0+.3390-0+.384$
}

Fig. $6.8 c$. The pressure in the various nodal points of a bearing half; $e_{x}=0.8, e_{y}=0$, $p_{\text {cav }}=-0.001$. 


\subsubsection{Effect of the parameter $p_{\mathrm{cav}}$ on a helical-groove bearing}

Figure 6.9 shows a bearing similar to that of fig. 6.6, but now with helical grooves. Grooves of this kind can be provided, for instance, to improve the stability under certain load conditions. It is to be expected that with such a bearing - as compared to the grooveless bearing shown in fig. 6.6 - the pumping effect of the grooves will assist the filling action from the edges.

That this is in fact the case may be seen from fig. 6.10. Here the total radial load capacity, $f$, of a grooved bearing is plotted as a function of the eccentricity $e_{x}$, with the grooved part $L_{g} / B$ of the bearing surface as parameter. Taking $p_{\text {cav }}$ $=0$, we find the emptying effect to be entirely suppressed if only $20 \%$ of the bearing surface is grooved. Similar results are found for different groove parameters.
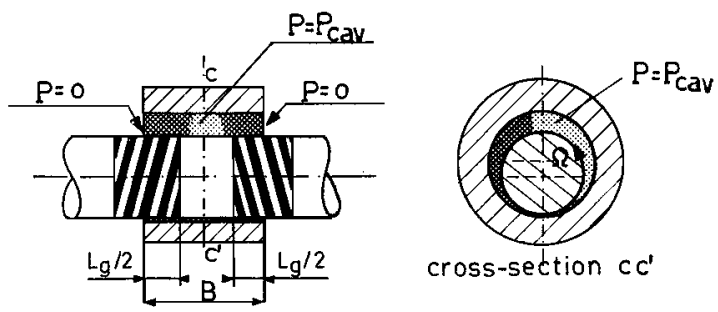

Fig. 6.9. Cavitation in a helically grooved cylindrical bearing.

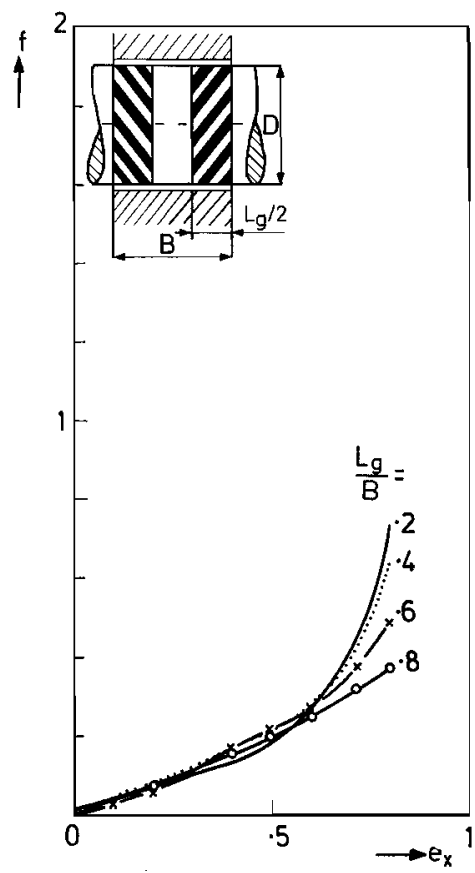

Fig. 6.10. The load capacity of a helically grooved cylindrical bearing $\left(B / D=1, e_{y}=0\right)$. 


\subsection{Dynamically loaded bearings}

The previous discussion on cavitation has been restricted to statically loaded bearings. For a given bearing, then, the region of cavitation is uniquely defined. by the position of the shaft and the parameter $p_{\text {cav }}$.

With a dynamically loaded bearing, at a certain point in time the region of cavitation is not uniquely defined, but the form and position of the region of cavitation basically depend upon the whole previous history of the bearing motion.

In general this creates unsurmountable problems for the calculation. In certain circumstances, however, we have a quasi-static situation, i.e. the dynamic effects are dominated by the static effects. Then the cavitation region may be calculated for the static load, and the influence of the small dynamic excursions around the static equilibrium position on the shape of the cavitation region may be neglected.

When the bearing-response coefficients are calculated (chapters 2, 3,4 and 7) the assumption of such a quasi-static situation has to be made, too. This is a consequence of the fact that for the linear stability theory it is necessary that the excursions, from a stationary equilibrium position, be so small that linearisation of the bearing response is permissible.

\section{REFERENCES}

6-1) B. Jakobson and L. Floberg, The finite journal bearing, considering vaporization, Trans. of Chalmers Univ. of Technology, Gothenburg Sweden, no. 190, 1957, report no. 3 from Inst. of Machine Elements.

6-2) R. Horsnell and H. McCallion, Predictions of some journal bearing characteristics under static and dynamic loading, Proceedings of the Lub. and Wear Conv. 1963, paper no. 12, pp. $126-138$.

${ }^{6-3}$ ) C. Y. Chow and J. H. Vohr, Helical-grooved journal bearing operated in turbulent regime, ASLE-ASME Lubr. Conference Houston, Texas (Oct. 1969), paper no. 69-Lub-28.

6-4) $H$. Sassenfeld and A. Walther, Gleitlagerberechnungen, VDI-Forschungsheft 441, edition B, vol. 20, 1954.

6-5) A. J. Smalley, T. Lloyd, R. Horsnell and H. McCallion, A comparison of performance predictions for steadily loaded journal bearings, Proc. Inst. mech. Engrs 180, 133-144, $1965 / 66$.

6-6) F. K. Orcutt and E. B. Arwas, The steady-state and dynamic characteristics of a full circular bearing and a partial arc bearing in the laminar and turbulent flow regimes, Trans. ASME, J. Lub. Techn. 89, 143-153, 1967.

6-7) J. A. Cole and C. J. Hughes, Oil flow and film extent in complete journal bearings, Proc. mech. Engrs 170, 499-510, 1956.

6-8) G. Vogelpohl, Beiträge zur Kenntnis der Gleitlagerreibung, VDI-Forschungsheft 386, edition B, vol, 8, Oct. 1937. 


\section{ACCURACY OF THE FINITE-ELEMENT METHOD AND RESULTS OF CALCULATIONS OF LOAD CAPACITY, RESPONSE COEFFICIENTS AND STABILITY OF VARIOUS TYPES OF BEARINGS}

\subsection{Introduction}

The theory developed in chapters 5 and 6 will be used here for the actual calculation of bearings. It is impossible, within the scope of this chapter, to give a complete survey of all the various kinds of bearings, and therefore the possibilities of the element method will be demonstrated by means of the calculated load capacity and bearing coefficients of a small number of bearing types that are of importance in engineering.

The versatility of the method will become clear as soon as it is realized that bearings with greatly differing geometries and boundary conditions can be calculated with the help of essentially the same basic program.

The outcome of a numerical computation is of practical use only if one knows the accuracy of the method. Now the finite-element method has the additional advantage of making a strong appeal to the sense of physics, so that great inaccuracies can readily be discerned.

If linear elements (see chapter 5) are used, then the inaccuracy increases if, in the case of a uniform distribution, the pressure gradient differs greatly from place to place. The approximation to the real pressure variation, and hence the accuracy, can be improved by making the elementary distributions finer; but then the requisite calculating time becomes longer. The choice of the elementary distribution will therefore have to be a compromise between calculating time and accuracy.

In the first part of this chapter the accuracy of calculation is analysed by comparison of the numerical results with cases which have been computed exactly as well as with generally accepted data from the literature which have been verified by experiments. In doing so, we shall adopt the criterion that, in view of the effect of the manufacturing tolerances, temperature, etc., on the performance of the bearing, an accuracy of calculation to within about $10 \%$ is adequate.

In the second part the load capacity and response coefficients of various kinds of bearings are calculated. To permit of an insight into the "stability" of a certain type of bearing, the stability of a symmetric rotor-and-bearing system, in which both the rotor and the bearing supports are rigid (theory of sec. 2.2), has been calculated. For some bearings it is shown that the assumption of rotationally symmetric response in the stability analysis (theory of chapter 3 ) leads to results that are sufficiently accurate. 


\subsection{Short description of the computer program}

In appendix III a computer program is given for the calculation of cylindrical bearings, with and without (rectangular) grooves. The main procedures of this program are used also in similar programs for flat and spherical spiralgroove bearings.

With this program it is possible to calculate bearings which have different groove patterns in three regions (fig. 7.1). The groove angle $\alpha$, the groove depth $h_{s}$, and the ridge-to-groove ratio $\gamma$ are represented in the program by the respective symbols fit, ho and gam.

The position of the three regions is determined by the values of $\Psi_{\min }, \Psi_{1}$, $\Psi_{2}$ and $\Psi_{\max }$ (in program: psimin, psil, etc.).

The deflection of the journal, relative to the bush, is determined by the dimensionless static deflection $\mathrm{e}(=\mathrm{E} / \Delta R)$ and dynamical deflection $\mathbf{x}(=\mathbf{X} / \Delta R)$. In the program the components of $e$ are represented by ex and ey and the components of the velocity, $\dot{\mathbf{x}}$, by eptx and epty.

The nodal points are chosen as a rectangular grid; the number of rows (along the circumference) equals $2 \times \mathrm{nfi}$ and the number of columns in the regions 1 , 2 and 3 is specified by nps1, nps2, and nps3.

In the first part of the program the coordinates of the nodal points are generated. Next, by means of the el procedure of appendix III, triangular elements (see sec. 5.5) are formed and the contribution of each element to the matrices of $(5.50)$ is determined with the genmatcilinder procedure.

The set of equations (5.24b), obtainable after application of the boundary conditions, may be resolved either by a direct method, the cholbd procedure, or by an iteration method, the iterate procedure. The solution then obtained,
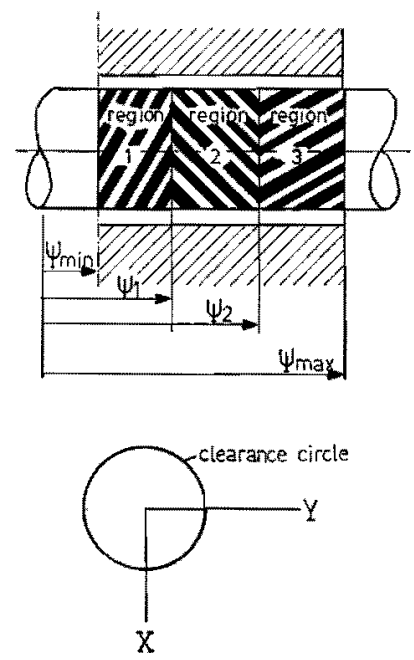

Fig. 7.1. Cylindrical bearing with different groove patterns in the regions, 1, 2 and 3 . 
the field of nodal pressures, is used in the genkracht procedure to calculate the dimensionless bearing reaction forces $f_{x}$ and $f_{y}$. Normally the input data eptx $=$ epty $=0$ are used and then the forces $f_{x}$ and $f_{y}$ represent the static reaction forces. By adding a small dynamical deflection to ex, etc., and repeating the calculation, we can find the data needed for the calculation of the response coefficients.

\subsection{Inaccuracies of the finite-element method}

\subsubsection{Sources of errors and principles for determining the inaccuracy}

The results obtained by a numerical method, such as the FEM, are of practical value only if the possible inaccuracy can be established. In this method several sources of inaccuracies can be distinguished.

Error type 1. The generalised Reynolds equation is, as a rule, applied to bearings with grooved surfaces. In sec. 5.4 mention is made of the errors resulting from the use of the generalised Reynolds equations instead of the normal one. Roughly speaking we may say here that, for example with a rectangular groove pattern, the error increases with increasing deviation from linear pressure profiles across the grooves and across the ridges.

Error type 2. When using the FEM the real pressure is approximated in the elements, for example, by a pressure which is a linear function of the coordinates (sec. 5.5). The accuracy that can be attained depends on the shape of the real pressure profile and on the possibility of approximating this pressure profile with the chosen functions: with linear functions the inaccuracy increases in general as the discontinuities, at the elementary boundaries, become greater. (but at a discontinuity in the film thickness the actual pressure gradient will be discontinuous and then an error is not necessarily introduced if the elementary boundary coincides with the discontinuity).

Error type 3. The FEM yields a set of linear inhomogeneous equations, from which the unknown nodal pressures have to be obtained. Depending on the method of solution used, which can be direct or iterative, an error may again be introduced here.

In predicting the inaccuracy of a numerical method it is common practice to compare the results that have been obtained from an exact calculation, or results that have been verified by measurements, with the results of that numerical method. The experience gained in this way makes it possible to estimate the error in the remaining cases. For this reason we shall now deal with some cases in which the results of the FEM can easily be checked. Here it should be noted that, on account of the great influence of the inevitable deviations in the geometry of a bearing in practice (manufacturing tolerances) on the pressure dis- 
tribution, the demanded accuracy should not be put too high, and an error of about $10 \%$ should be regarded as acceptable.

\subsubsection{Comparison with the Sommerfeld and the Reynolds solutions}

For an infinitely long bearing $(B=\infty)$ exact solutions are known for two types of boundary conditions: with the Sommerfeld conditions the assumption is made of $P=0$ at $\Phi=0$ and at $\Phi=\pi$ (fig. 6.1), while with the Reynolds conditions we assume $P=0$ at $\Phi=0$ at the beginning of the pressure hill, and at its end it is required that $P=0$ and $\mathrm{d} P / \mathrm{d} \Phi=0$ (Pinkus et al. ${ }^{7-1}$ )).

In figs 7.2 and 7.3 the exact pressure is represented by a fully drawn curve; the values numerically calculated with the FEM are indicated, for 16, 32 and 64 nodal points in the circumferential direction, by respectively black circles, crosses and open circles.

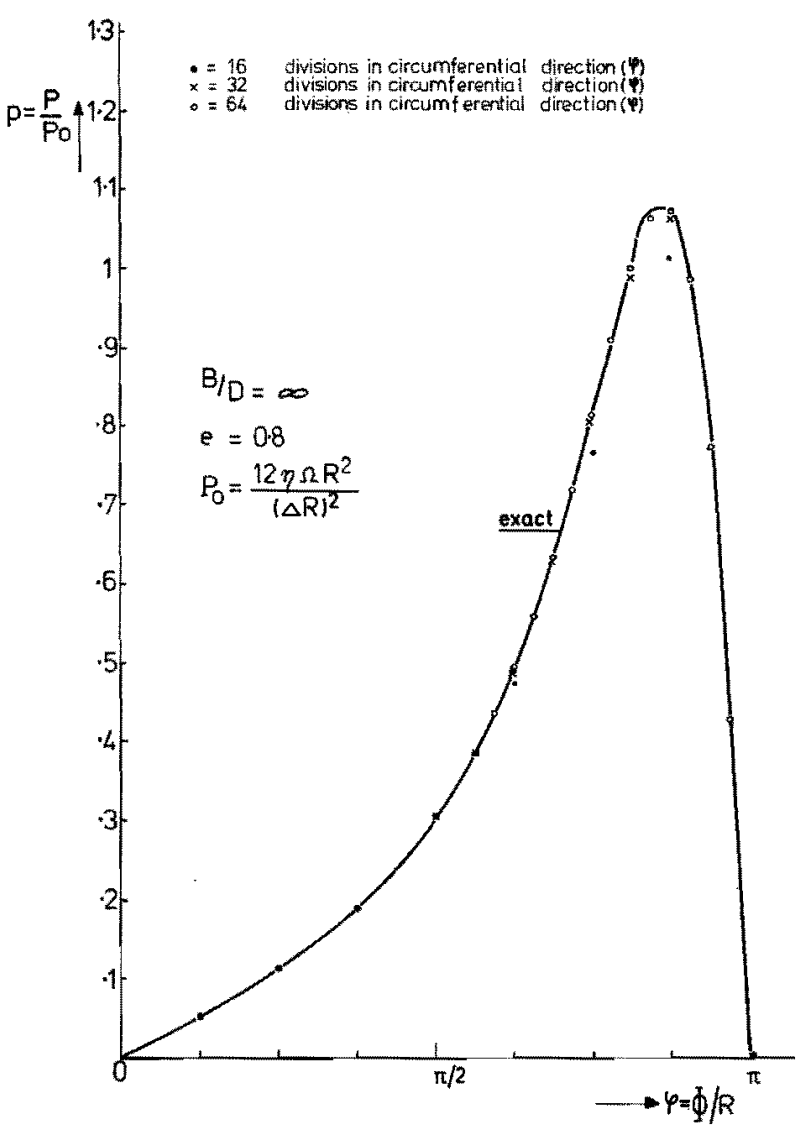

Fig. 7.2. Influence of the number of grid points on the calculated pressure; Sommerfeld's boundary condition. 


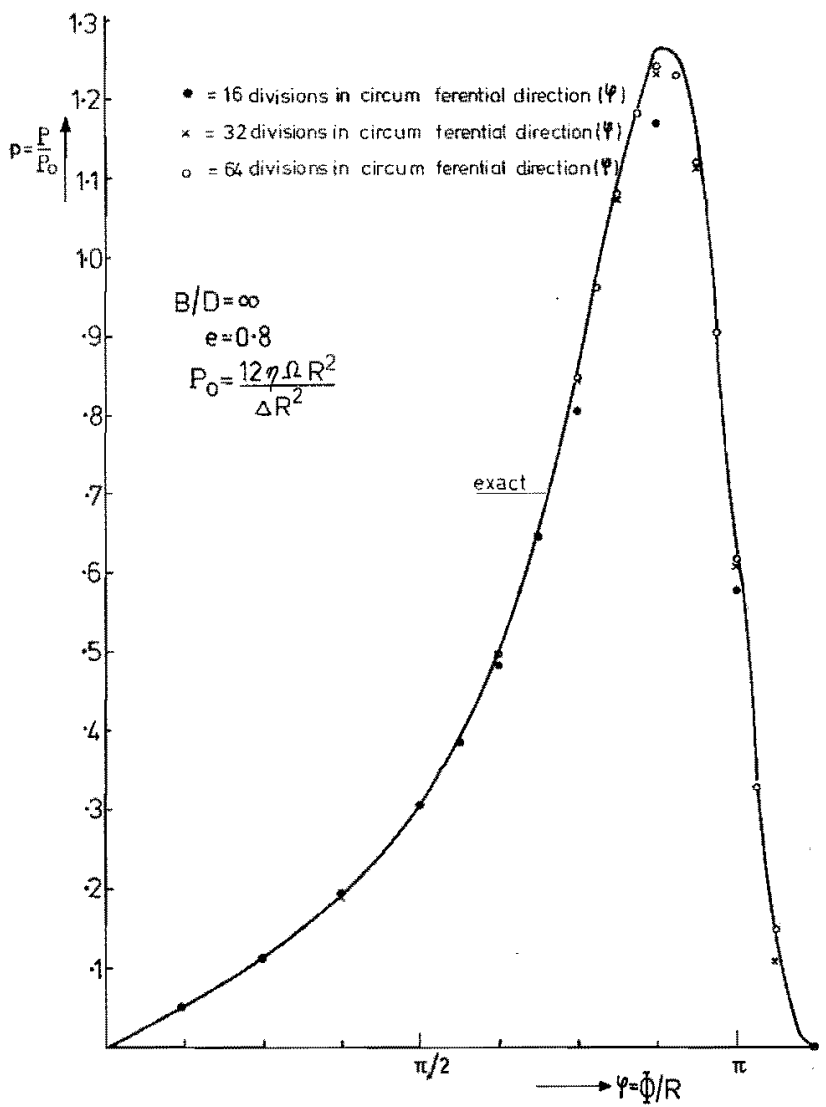

Fig. 7.3. Infuence of the number of grid points on the calculated pressure; Reynolds' boundary condition.

The greatest departures occur at the maximum pressure, where the pressure gradient shows the greatest rate of variation. In any case, even with the coarse distribution of 16 grid points, the deviation is less than $10 \%$. Owing to the averaging effect, due to the integration of the pressure, the error in the load capacity will be even smaller. This is seen from table 7-I, in which the load capacity and attitude angle are recorded.

\subsubsection{Comparison with the results of Sassenfeld and Walther}

The data on the load capacity of smooth cylindrical journal bearings presented by Sassenfeld and Walther ${ }^{7-2}$ ) are frequently used for engineering purposes and have been verified experimentally. They can therefore be used for checking the accuracy of the FEM.

Table 7-II embodies the results of Sassenfeld and Walther and those obtained with the FEM. In the FEM programme the distribution with 32 nodal points 


\section{TABLE 7-I}

Load capacity and attitude angle of a section with length $R$ of an infinite journal bearing. Influence of the number of nodal points on the accuracy;

$$
F=f F_{0}, \quad F_{0}=\frac{12 \eta \Omega R^{4}}{(\Delta R)^{2}}
$$

\begin{tabular}{|c|c|c|c|c|c|}
\hline \multirow{3}{*}{$\begin{array}{l}\text { number of } \\
\text { nodal points } \\
\text { along circum- } \\
\text { ference }\end{array}$} & \multicolumn{2}{|c|}{$\begin{array}{l}\text { Sommerfeld } \\
\text { conditions }\end{array}$} & \multicolumn{2}{|c|}{$\begin{array}{c}\text { Reynolds } \\
\text { conditions }\end{array}$} & \multirow{3}{*}{$\begin{array}{l}\text { eccen- } \\
\text { tricity }\end{array}$} \\
\hline & $\begin{array}{c}\text { load } \\
\text { capacity }\end{array}$ & $\begin{array}{c}\text { att. } \\
\text { angle }\end{array}$ & $\begin{array}{c}\text { load } \\
\text { capacity }\end{array}$ & $\begin{array}{c}\text { att. } \\
\text { angle }\end{array}$ & \\
\hline & $f$ & $\varphi_{a}(\mathrm{rad})$ & $f$ & $\varphi_{a}(\mathrm{rad})$ & \\
\hline 16 & $1 \cdot 52$ & $1 \cdot 57$ & $1 \cdot 18$ & $\cdot 74$ & .8 \\
\hline 32 & $1 \cdot 57$ & 1.57 & $1 \cdot 23$ & .74 & .8 \\
\hline 64 & $1 \cdot 58$ & $1 \cdot 57$ & $1 \cdot 24$ & .74 & $\cdot 8$ \\
\hline exact solution & 1.59 & $\pi / 2$ & $1 \cdot 26$ & .74 & .8 \\
\hline
\end{tabular}

\section{TABLE 7-II}

Comparison of the results of Sassenfeld and Walther with those of the FEM. $B / D=1 ; 32$ nodal points in circumferential direction; 6 nodal points in axial direction for a half bearing; computer program: appendix III;

$$
F=f F_{0} ; \quad F_{0}=\frac{12 \eta \Omega R^{4}}{(\Delta R)^{2}}
$$

\begin{tabular}{c|c|c|c|c}
\hline \multirow{2}{*}{$\begin{array}{c}\text { eccentricity } \\
e\end{array}$} & \multicolumn{2}{|c|}{ Sassenfeld-Walther } & \multicolumn{2}{c}{ finite-el. method } \\
\cline { 2 - 5 } & $\begin{array}{c}\text { load cap. } \\
f\end{array}$ & $\begin{array}{c}\text { att. angle } \\
\varphi_{a} \text { (rad) }\end{array}$ & $\begin{array}{c}\text { load cap. } \\
f\end{array}$ & $\begin{array}{c}\text { att. angle } \\
\varphi_{a}(\mathrm{rad})\end{array}$ \\
\hline 0.1 & 0.039 & 1.38 & 0.039 & 1.38 \\
0.2 & 0.084 & 1.29 & 0.083 & 1.29 \\
0.3 & 0.139 & 1.19 & 0.134 & 1.19 \\
0.4 & 0.203 & 1.08 & 0.200 & 1.09 \\
0.5 & 0.297 & 0.98 & 0.290 & 0.99 \\
0.6 & 0.441 & 0.87 & 0.428 & 0.88 \\
0.7 & 0.693 & 0.75 & 0.660 & 0.77 \\
0.8 & 1.18 & 0.63 & 1.13 & 0.64 \\
0.9 & 2.78 & 0.44 & 2.62 & 0.46 \\
\hline
\end{tabular}


along the circumference and 6 nodal points along the axis is used. On account of the symmetry, only a half bearing had to be calculated. The departures are found to increase with the eccentricity. This can be explained by the fact that here FEM uses linear elements, which are susceptible to the strong changes in pressure gradient occurring at large eccentricities (the difference method utilised by Sassenfeld and Walther is a "quadratic" approximation). Since the deviation, even at an eccentricity of 0.9 , is less than $6 \%$, a distribution of $32 \times 6$ is considered to be sufficiently "fine".

\subsubsection{The accuracy of the pressure build-up in a grooved, centric journal bearing}

If a grooved bearing (fig. 7.1) is in the concentric position, the pressure profile on the groove pattern is linear. This means that the linear elements used can represent the real pressure variation exactly, and the effect of type-1 and type-2 errors vanishes.

We have made a comparison with the analytical results of Muijderman ${ }^{7-3}$ ): if the direct resolution method (cholbd procedure) is used, the error is found to be negligibly small $(<0.1 \%)$, irrespective of the number and shape of the elements.

\subsubsection{Comparison with analytical results of a spherical spiral-groove thrust bear- ing}

The geometry of this type of bearing is much more complicated than that of a journal bearing. Nevertheless the same basic procedures can be used in the calculation by means of the FEM. In sec. 7.4.5 this type of bearing will be treated in greater detail and for the definitions of the geometric parameters and illustrations the reader is referred to that section.

In the present section an impression of the accuracy of the calculation has been gained by comparison of the FEM results with the exact value obtained by Muijderman ${ }^{7-3}$ ) in the particular case of the concentric position of the sphere in the cup $\left(e_{x}=e_{y}=e_{z}=0\right)$ and an infinite number of grooves $\left(k_{g}=\infty\right)$. The results are listed in table 7-III. For the groove parameters, use was made of the values which were optimum for the axial load capacity ${ }^{7-3}$ ).

It was assumed in the FEM calculation that the sphere was smooth from $\Psi=0$ to $\Psi=\Psi_{\min }$ and grooved from $\Psi_{\min }$ to $\Psi_{\max }$. This departs from ref. $7-3$, where $\Psi_{\min }$ was taken to be zero, but this of course cannot be realised in practice. From table 7-III it can be deduced that $\Psi_{\min }$ has a relatively slight effect on the load capacity. Even for $\Psi_{\min }=0.6 \mathrm{rad}$ (case No. 1) the deviation is as little as $7 \%$. This is in agreement with expectations, bearing in mind that the groove pattern at the "south pole" rotates at a comparatively slow speed and contributes but little to the pressure build-up and the load capacity. The fact that for $\Psi_{\min }=0.1$ a slightly lower value for $f_{z}$ is found is attributable to the fact that the shape of the elements at low values of $\Psi_{\text {min }}$ becomes more 


\section{TABLE 7-III}

Discrepancy between calculated axial thrust and exact value for a concentric spherical thrust bearing.

$$
\begin{array}{ll}
\alpha=0.28 \mathrm{rad}, & \Psi_{\min }-\Psi_{\max }: \text { grooved, } \\
h_{s}=2 \cdot 6, & e_{x}=e_{y}=e_{z}=0, \\
\gamma=1, & k_{g}=\infty, \\
0-\Psi_{\min }: \text { grooveless, } & \\
\qquad F_{z}=f_{z} F_{0} ; & F_{0}=\frac{12 \eta \Omega R^{4}}{(\Delta R)^{2}},
\end{array}
$$

$\mathrm{nfi}=$ number of nodal points on a parallel of latitude, npsi $=$ number of nodal points from $\Psi_{\min }$ to $\Psi_{\max }$,

\begin{tabular}{c|c|c|c|c|c}
\hline number & nfi & npsi & $\begin{array}{l}\Psi_{\min } \\
\text { (rad) }\end{array}$ & $\begin{array}{l}\Psi_{\max } \\
\text { (rad) }\end{array}$ & $f_{z}$ \\
\hline 1 & 32 & 4 & 0.6 & 1.57 & 0.0893 \\
2 & 32 & 6 & 0.4 & 1.57 & 0.0918 \\
3 & 32 & 6 & 0.2 & 1.57 & 0.0919 \\
4 & 32 & 6 & 0.1 & 1.57 & 0.0915 \\
5 & 32 & 8 & 0.1 & 1.57 & 0.0928 \\
6 & 32 & 10 & 0.1 & 1.57 & 0.0934 \\
7 & \multicolumn{5}{c}{ exact value (Muijderman) } \\
\hline
\end{tabular}

unfavourable, so that the error will increase. From No. 4, No. 5, and No. 6 in the table it is seen that a distribution with 6 nodal points from $\Psi_{\min }$ to $\Psi_{\max }$ provides sufficient accuracy. The requisite number of distributions in the circumferential direction is difficult to evaluate with this concentric position, as it is precisely the changes in the pressure gradients (which increase in the case of an eccentric position) that introduce inaccuracies into the calculation. The choice of 32 nodal points in the circumferential direction for each calculation is therefore based on the insights gained from experience with journal bearings.

\subsection{Load capacity, response coefficients and stability diagrams of some types of bearings}

\subsubsection{Determination of the response coefficients}

In what follows, the load capacity, the response coefficients calculated with the FEM, and the stability curves of several types of bearings calculated with the theory of chapter 2 are given. 
The calculation of the dynamic coefficients requires some furhter explanation. It will be inferred from (2.12) that, for example, $a_{x x}$ and $a_{y x}$ allow of being determined by a displacement of the bearing through a small distance $x$ from the equilibrium position $x=0$ : this results in increments $\Delta f_{x}$ and $\Delta f_{y}$ of respectively $f_{x}$ and $f_{y}$, so that we have:

$$
a_{x x}=-\frac{\Delta f_{x}}{x} ; \quad a_{y x}=-\frac{\Delta f_{y}}{x} .
$$

Analogously $a_{y y}$ and $a_{x y}$ are determined with the aid of a displacement $y, b_{x x}$ and $b_{y x}$ are determined with the aid of a velocity $\dot{x}, b_{y y}$ and $b_{x y}$ are determined with the aid of a velocity $\dot{y}$.

The displacements $x$ and $y$ should be so small that the effect on $f_{x}$ and $f_{y}$ remains linear, in other words $\mathrm{d} f_{x} / \mathrm{d} x, \mathrm{~d} f_{y} / \mathrm{d} x$, etc., will be adequately approximated by $\Delta f_{x} / x, \Delta f_{y} / x$, etc.

The Reynolds equation implies that $f_{x}$ and $f_{y}$ are linear functions of $\dot{x}$ and $\dot{y}$, so that $\dot{x}$ and $\dot{y}$ do not have to be small to yield an accurate result. It is in fact better to take them fairly large in order to arrive at the desired numerical accuracy.

The occurrence of cavitation complicates matters: the shape and size of the cavitation region will then also depend on $x, \dot{x}$, etc. In a dynamically loaded bearing the cavitation region depends on the instantaneous situation (determined by $\mathbf{x}$ and $\dot{\mathbf{x}}$ ) as well as on its whole history.

In the publications with which the author is familiar this problem is solved by the assumption of a quasi-stationary situation when determining the region of cavitation. Then $\mathbf{x}$ and $\dot{\mathbf{x}}$ must be small. On the assumption, however, that this quasi-stationary situation does in fact govern the cavitation region, it is also justified to adopt the situation $\mathbf{x}=\dot{\mathbf{x}}=0$ for the iteration of the cavitation region and, when $a_{x x}$ and so on are determined, subsequently to fix the boundary of the cavitation region (with $p=p_{\text {cav }}$ as the boundary condition). Thus the calculation is speeded up, since the recurring iteration of the cavitation region, for each increment, can be omitted. Besides, $\dot{x}$ need no longer be small.

\subsubsection{Smooth journal bearing with axial lubricant-supply groove (ALG bearing)}

This configuration has been used by Sassenfeld and Walther in their calculations ${ }^{7-2}$ ). It is assumed that this type of journal bearing possesses an Axial Lubricant-supply Groove (ALG) at the location of the widest film gap $(\Phi=0)$. In this groove, in the cavitation region, and at the edges of the bearing $P$ is put equal to zero (fig. 7.4). At the same time this groove, which supplies the lubricant required for continuity of flow, forms the trailing edge of the cavitation region. 


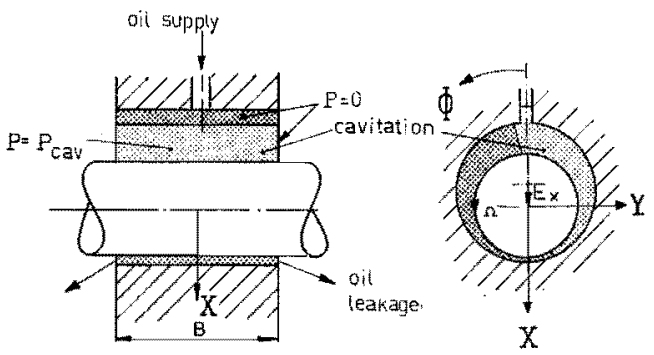

Fig. 7.4. An ALG bearing.

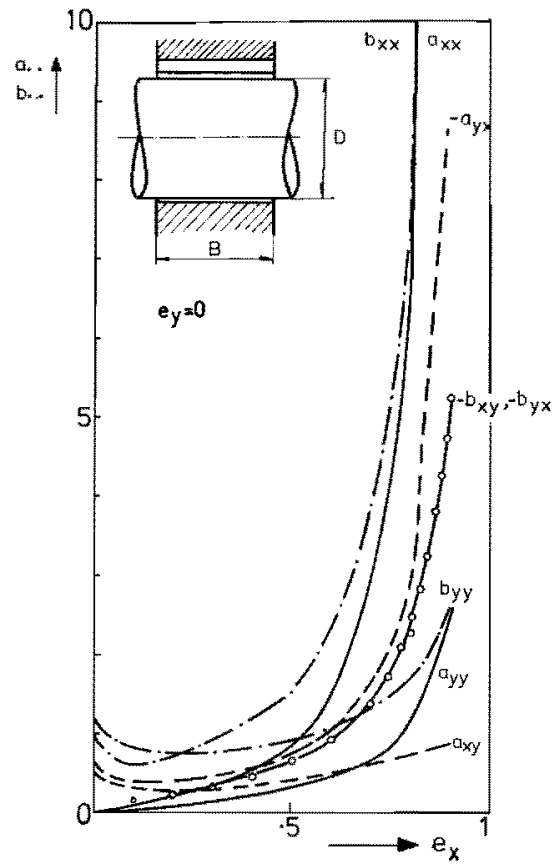

Fig. 7.5. Response coefficients of an ALG bearing $\left(B / D=1, p_{\text {cav }}=0\right)$.

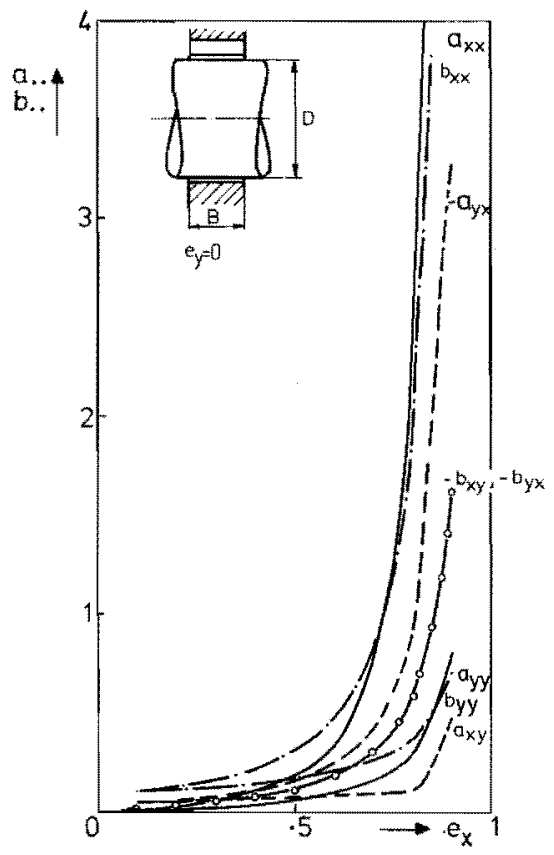

Fig. 7.6. Response coefficients of an ALG bearing $\left(B \mid D=0.5, p_{\text {cap }}=0\right)$.

Figures 7.5 and 7.6 give, for respectively $B / D=1$ and $B / D=0.5$, the response coefficients needed for the calculation of the stability. With $F_{0}=$ $12 \eta \Omega R^{4} /(\Delta R)^{2}$ these coefficients conform to

$$
a_{x x}=\frac{\Delta R}{F_{0}} A_{x x} ; \quad a_{x y}=\frac{\Delta R}{F_{0}} A_{x y} ; \quad b_{x x}=\frac{\Delta R \Omega}{F_{0}} B_{x x} ; \text { etc. }
$$

Figures 7.7 and 7.8 show the load-capacity and the stability curves, relating to the rotor described in sec. 2.2, for the bearings of figs. 7.5 and 7.6.

This bearing is not rotationally symmetrical on account of the presence of the groove, so that it cannot be proved that the bearing is inherently unstable 


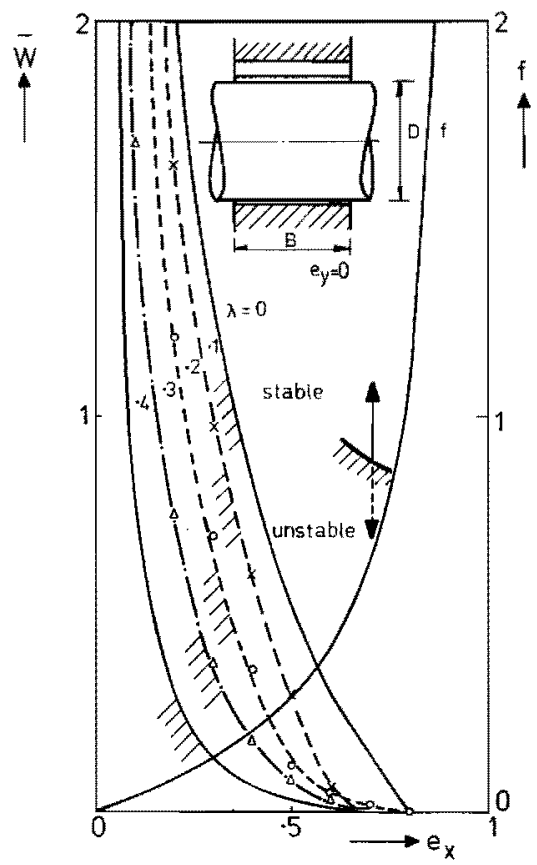

Fig. 7.7. Stability curves and load-carrying capacity of an ALG bearing $(B / D=1$, static load).

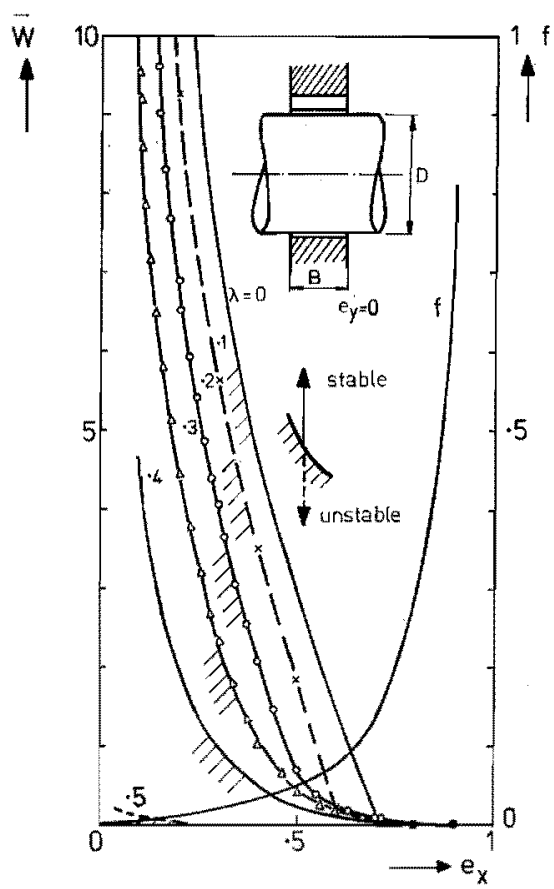

Fig. 7.8. Stability curves and load-carrying capacity of an ALG bearing $(B / D=0.5$, static load).

at an eccentricity $e_{x}=0$ (and $\lambda=0$ ), but in practice this is certainly the case. For $\lambda=0$ and $e_{x}>0.8$ the bearing is inherently stable (see also chapter 2), as it is also for $\lambda>\mathrm{ca} 0.5$. The load capacity in the two figures is in complete agreement with ref. $7-2$.

It appears that both the load capacity and the dynamic coefficients remain relatively small at low values of the eccentricity $\left(e_{x}<0.8\right)$ and increase rapidly at higher values of $e_{x}$, which explains the good stability at the last-mentioned values.

\subsubsection{Smooth journal bearing with circumferential oil supply (CLG bearing)}

This type of bearing is in fact an ALG bearing, but without an axial groove (see fig. 7.9). At its edges $(\Psi= \pm B / 2)$ the lubricant has a pressure $P=0$. If the lubricant cavitates at a pressure- $P_{\text {cav }}$, then the pressure difference represented by $0-P_{\text {cav }}$ is the determining factor affecting the oil supply to the bearing.

A description of an investigation of the effect of the cavitation pressure on the load capacity has already been given in sec. 6.4.1. In fig. 7.10 the responce coefficients are plotted against $e_{x}$ for $p_{\text {cav }}=P_{\text {cav }} / P_{0}=-0.01$. The differences from the ALG bearing (cf. fig. 7.5) are not very great. The stability 

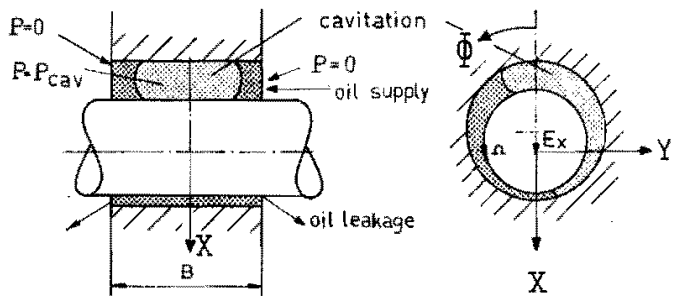

Fig. 7.9. A CLG bearing.

curves also give a similar picture (cf. fig. 7.11). But at $p_{\text {cav }}=-0 \cdot 1$ (figs 7.12 and 7.13) the picture is quite different: the occurrence of cavitation is now strongly suppressed, causing the coeficients $a_{x x}$ and $a_{y y}$ for small values of $e_{x}\left(e_{x}<0.4\right)$ to tend to zero (fig. 7.12), which affects the stability curves in such a way that for $e_{x}<0.4$ the bearing virtually becomes inherently unstable (fig. 7.13). This was to be expected, since according to the theory given in chapters 2 and 3 a noncavitating smooth bearing is always unstable (for example in a bearing with rotationally symmetric response: $a=0$ ).

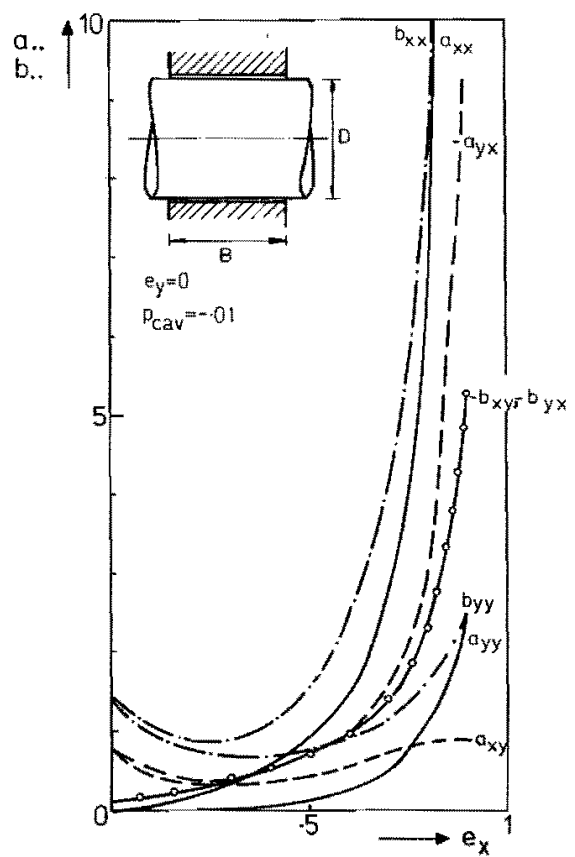

Fig. 7.10. Response coefficients of a CLG bearing ( $B / D=1, p_{\mathrm{car}}=-0.01$ ),

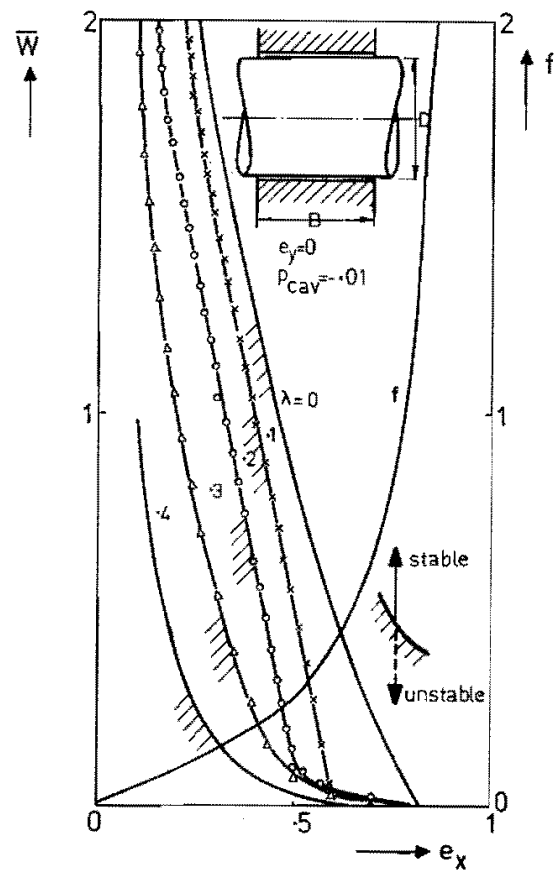

Fig. 7.11. Stability curves and load-carrying capacity of a CLG bearing $(B / D=1$, static load). 


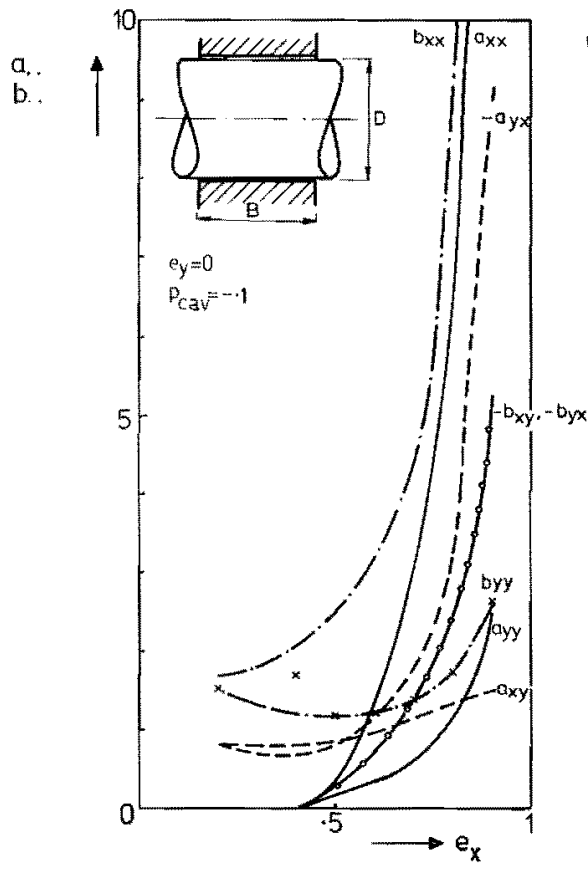

Fig. 7.12. Response coefficients of a CLG bearing $(B / D=1)$.

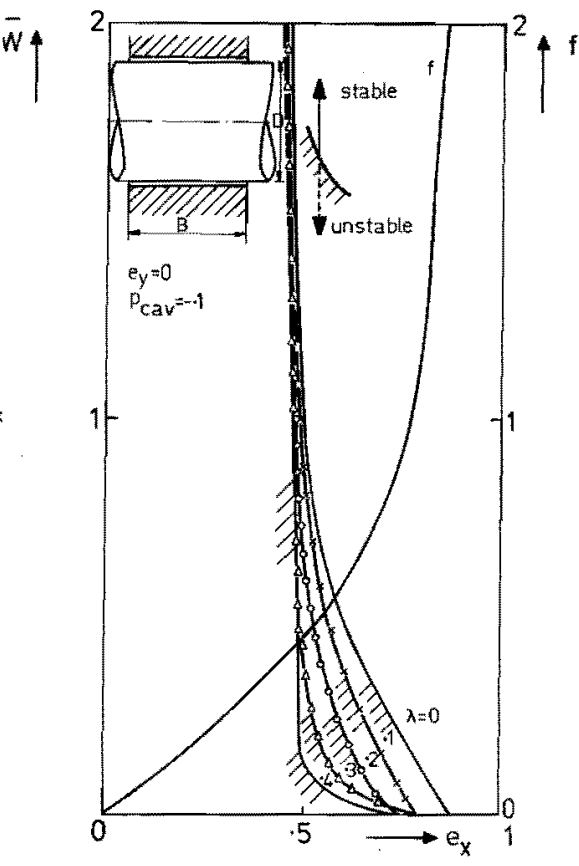

Fig. 7.13. Stability curves and load-carrying capacity of a CLG bearing $(B / D=1$, static load).

Note. An exact determination of the cavitation region is all-important, since the stability of a rotor in grooveless bearings (chapters 2 and 3 ) can be achieved solely by cavitation. If, in the case $p_{\mathrm{cav}}=-0.1$, the data of the ALG bearing were used, then a wrong result would be obtained. Hence the cavitation region has a considerable influence not only on the load capacity (see chapter 6) but also on the response coefficients.

\subsubsection{Grooved journal bearing with optimum radial stiffness (ORS bearing)}

The previously discussed grooveless bearings possess poor stability at small eccentricities. This is bound up with the fact that the "attitude angle", $\varphi_{a}$ (fig. 7.14), is large. The $x$-component of the bearing reaction consequently becomes small and, on that account, $a_{x x}$ and $a_{y y}$ become small. Because $a_{x x}$ and $a_{y y}$ are the predominant factors determining the stability (compare with the " $a$ " of chapter 3 ) this leads to poor stability.

The large value of $\varphi_{a}$ is due to the fact that the smooth bearings derive their load capacity from the pressure build-up in the wedge-shaped gap (in the absence of cavitation such a pressure field implies that $\varphi_{a}=\pi / 2$ ). This phenomenon we shall call the pressure field due to the "wedge effect" (fig. 7.15a). As the eccentricity increases, $\varphi_{a}$ then becomes smaller, owing to cavitation, and 

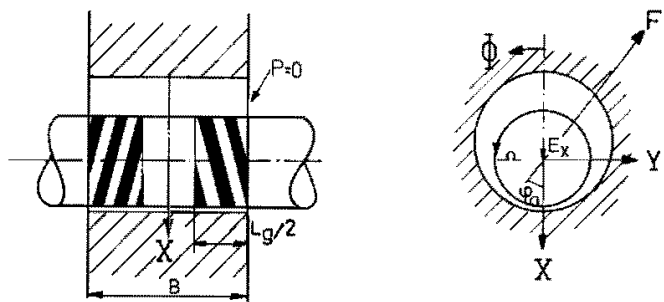

Fig. 7.14. Helically grooved journal bearing.

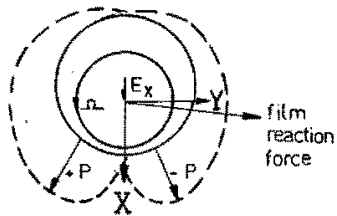

a)

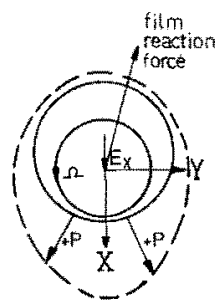

b)

Fig. 7.15. The wedge and groove effects. $(a)$ : Pressure field due to wedge effect; $(b)$ : pressure field due to groove effect.

the stability improves. It is also a well-known fact that the pressure field due to the wedge effect vanishes if the vector $\mathbf{E}$ rotates at a velocity $\Omega / 2$.

With a grooved bearing (fig. 7.14), besides the wedge effect a "groove effect" can be distinguished also. The pressure field due to the groove effect (fig. 7.15b) is produced (in the case of a correct design) because of the fact that the grooves in the narrow gap induce, at $\Phi=\pi$, a larger pressure gradient in the axial direction than they do in the wide gap at $\Phi=0$. Thus a pressure field profile with a shape as shown in fig. $7.15 b$ is generated inside the bearing. As this pressure distribution is more or less symmetrical with respect to $\Phi=\pi$, the force of reaction is practically opposite in direction to the displacement $E_{x}$; this is precisely the situation which favours stability (a grooved journal bearing is, as it were, "hydrodynamically prestressed").

If a grooved bearing whirls at the characteristic frequency, $\delta_{c}$, this means that $\mathbf{E}$ rotates with an angular velocity of ca $\Omega / 2\left(\delta_{c} \approx 0.5\right)$ and also then the wedge effect will be small: the load capacity will be generated by the groove effect. For the system of chapter 3 , in which the stabitity is governed by the load capacity at the characteristic frequency, we may say that this stability is almost exclusively determined by the groove effect.

Chow and Vohr ${ }^{7-4}$ ) have determined the geometric parameters of a bearing with an Optimum Radial Stiffness (ORS bearing) in the case of a concentric position of the shaft. This type of bearing (fig. 7.14) has two grooved ends and a smooth part in the middle. 
The optimum parameters are, for the case of $k_{g}=\infty$ :

groove depth

groove angle

ridge-to-groove ratio

fraction grooved

$$
\begin{aligned}
& h_{0}=1 \cdot 1, \\
& \alpha=0.5 \mathrm{rad}, \\
& \gamma=1, \\
& L_{g} / B=0.75
\end{aligned}
$$

The boundary conditions are:

$$
\begin{aligned}
& P=0 \text { for } \Psi= \pm B / 2, \\
& P_{\text {cav }}=p_{\text {cav }}=0 .
\end{aligned}
$$

As found above, the choice of the cavitation condition has little influence on the load capacity. That this is also applicable to the type of bearing under discussion may be seen from table 7-IV, which gives the load capacity and the attitude angle for cavitation condition 1 (also used by Chow and Vohr ${ }^{7-4}$ )) and cavitation condition 4. Table 7-IV in addition shows that the response coefficients are slightly different. In figs 7.16 and 7.17 the response coefficients are plotted against $e_{x}$ for condition 4. Figures 7.18 and 7.19 show the loadcapacity and the stability curves in respect of a rotor for which the theory of chapter 2 is applicable. The difference between the two cavitation conditions becomes manifest only at $e_{x}=0.6$ to 0.8 .

\begin{tabular}{|c|c|c|c|c|c|c|c|c|c|c|c|}
\hline $\begin{array}{l}\text { cav. } \\
\text { cond. }\end{array}$ & $e_{x}$ & $a_{x x}$ & $a_{y y}$ & $a_{x y}$ & $a_{y x}$ & $b_{x x}$ & $b_{y y}$ & $b_{x y}$ & $b_{y x}$ & $\varphi_{a}$ & $f$ \\
\hline $\begin{array}{l}1 \\
4\end{array}$ & 0 & $+\cdot 200$ & $+\cdot 200$ & $+\cdot 301$ & --301 & $+\cdot 591$ & +.591 & -.004 & +.004 & 980 & 0 \\
\hline $\begin{array}{l}1 \\
4\end{array}$ & 0.2 & $+\cdot 217$ & $+\cdot 206$ & $+\cdot 306$ & $-\cdot 318$ & $+\cdot 638$ & $+\cdot 603$ & -.001 & +.007 & .980 & .074 \\
\hline 4 & 0.4 & $\begin{array}{l}+\cdot 313 \\
+\cdot 289\end{array}$ & $\begin{array}{l}+\cdot 213 \\
+\cdot 234\end{array}$ & $\begin{array}{l}+\cdot 265 \\
+\cdot 265\end{array}$ & $\begin{array}{l}-\cdot 225 \\
-\cdot 242\end{array}$ & $\begin{array}{l}+\cdot 668 \\
+\cdot 674\end{array}$ & $\begin{array}{l}+\cdot 358 \\
+\cdot 388\end{array}$ & $\begin{array}{l}-.055 \\
-.018\end{array}$ & $\begin{array}{l}-.048 \\
-.010\end{array}$ & $\begin{array}{l}.939 \\
.903\end{array}$ & $\begin{array}{r}\cdot 154 \\
\cdot 156\end{array}$ \\
\hline 4 & 0.6 & $\begin{array}{l}+\cdot 526 \\
+\cdot 504\end{array}$ & $\begin{array}{l}+\cdot 255 \\
+\cdot 259\end{array}$ & $\begin{array}{l}+\cdot 212 \\
+\cdot 288\end{array}$ & $\begin{array}{l}-.295 \\
-.299\end{array}$ & $\begin{array}{l}+\cdot 787 \\
+\cdot 831\end{array}$ & $\begin{array}{l}+\cdot 361 \\
+\cdot 364\end{array}$ & $\begin{array}{l}-\cdot 153 \\
-\cdot 143\end{array}$ & $\begin{array}{l}-\cdot 144 \\
-\cdot 134\end{array}$ & $\begin{array}{l}\cdot 828 \\
\cdot 745\end{array}$ & $\begin{array}{l}.244 \\
.250\end{array}$ \\
\hline 4 & 0.8 & $\begin{array}{l}+1.05 \\
+1.04\end{array}$ & $\begin{array}{l}+\cdot 347 \\
+\cdot 338\end{array}$ & $\begin{array}{l}+\cdot 193 \\
+\cdot 236\end{array}$ & $\begin{array}{l}-.460 \\
-.470\end{array}$ & $\begin{array}{l}+1 \cdot 30 \\
+1 \cdot 41\end{array}$ & $\begin{array}{l}+\cdot 410 \\
+\cdot 383\end{array}$ & $\begin{array}{l}-\cdot 314 \\
-\cdot 314\end{array}$ & $\begin{array}{l}-.297 \\
-\cdot 297\end{array}$ & $\begin{array}{l}.709 \\
.620\end{array}$ & $\begin{array}{l}\cdot 392 \\
\cdot 398\end{array}$ \\
\hline
\end{tabular}

There is a remarkable difference between the stability curves for a smooth bearing (for example fig. 7.7) and those for a grooved bearing (fig. 7.18). The

\section{TABLE 7-IV}

The response coefficients, attitude angle and load capacity of an ORS bearing $(B / D=1)$ 


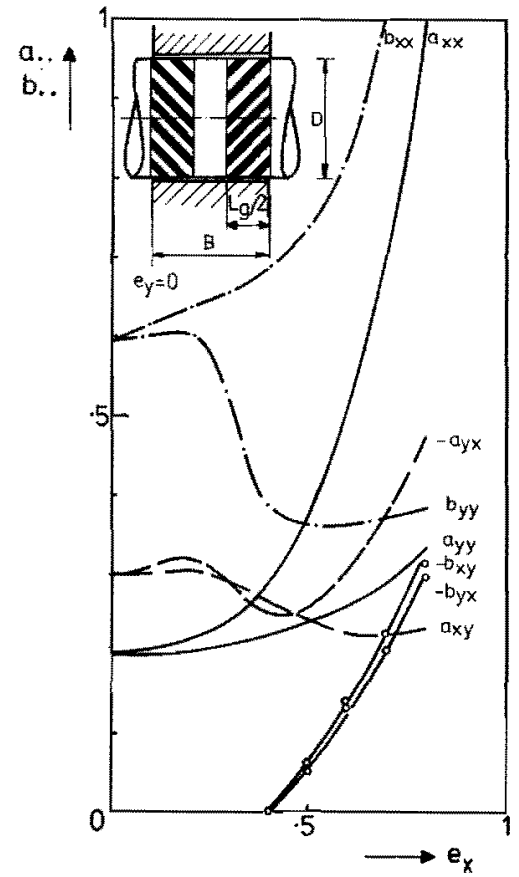

Fig. 7.16. Response coefficients of an ORS bearing $(B / D=1)$.

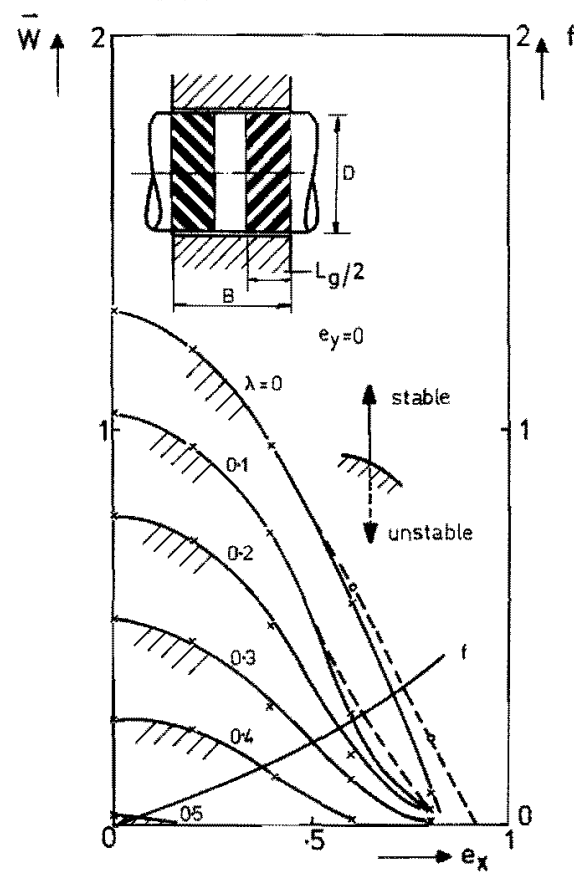

Fig. 7.18. Stability curves and load-carrying capacity of an ORS bearing $(B / D=1$, static load) _ $\times \ldots$ cavitation condition 1 ; ..- o-..- cavitation condition 4 (J \& F).

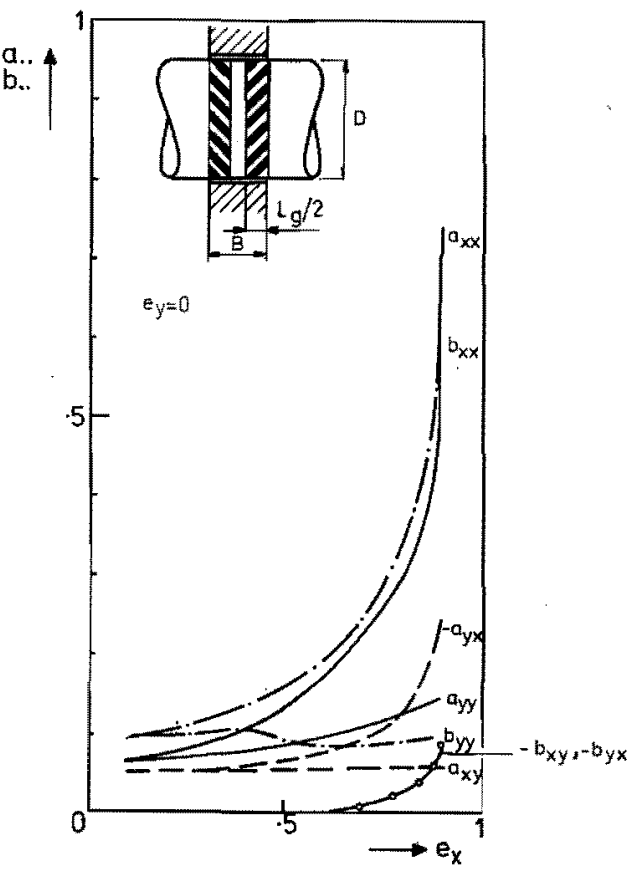

Fig. 7.17. Response coefficients of an ORS bearing $(B / D=0.5)$,

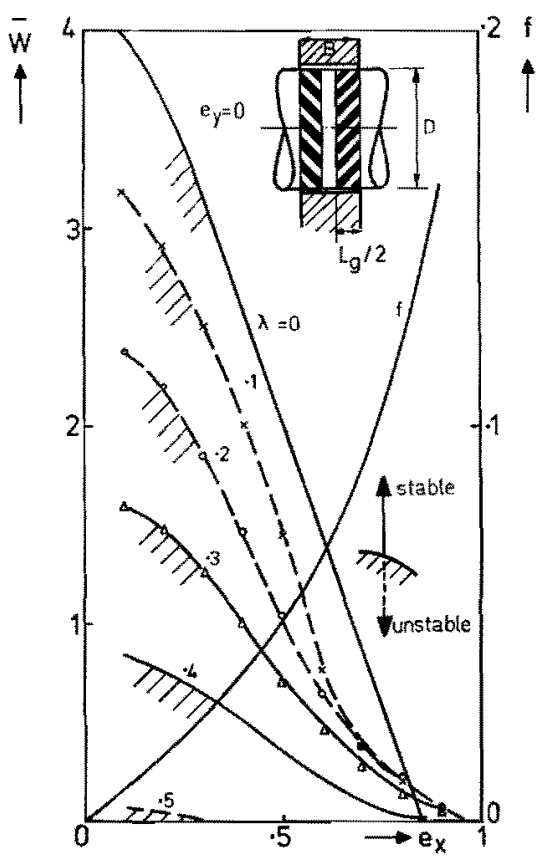

Fig. 7.19. Stability curves and load-carrying capacity of an ORS bearing $(B / D=0.5$, static load). 
smooth bearing is inherently unstable at small values of the eccentricity, whereas the grooved bearing exhibits satisfactory stability properties even if $e_{x}=0$. But at $e_{x}>0.3$ the smooth bearing (e.g. $\lambda=0$ ) is distinctly superior. The same applies to the load capacity.

The designer can apply the following criterion to good advantage:

- if a bearing has to work under the influence of a static load at small eccentricities (say $e_{x}<0.3$ ) then it is better, in view of the load capacity and the stability, to select the grooved bearing (fig. 7.14);

- if, on the other hand, the bearing has to work at bigger eccentricities, then a smooth bearing (fig. 7.4) is preferable.

Note. In the case of a limit cycle (chapter 3 ) being employed, the groove pattern referred to above will not be optimum and a new optimisation of the parameters should be performed.

\subsubsection{The spherical spiral-groove bearing}

One of the most interesting features of a spherical spiral-groove bearing is that it is capable of taking up a radial load as well as an axial load. Although the radial load capacity is less than the axial load capacity, the former can nevertheless be up to $25 \%$ of the latter, provided that the bearing is properly designed. Another favourable property of this bearing is that it does not have to be aligned because it can rotate, as it were, as a ball joint about the centre of the sphere.

In fig. 7.20 the geometric parameters of the bearing are defined. The angle $\alpha$ between the grooves and the parallel of latitude is measured in a plane tangent to the sphere.

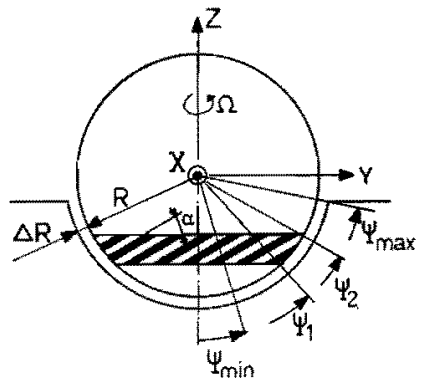

Fig. 7.20. Geometric parameters of a spherical spiral-groove bearing (drawn in the concentric position).

$h_{s}=$ groove depth/radial clearance $\left(=H_{s} / \Delta R\right)$;

$\alpha=$ angle between grooves and parallel of latitude;

$\gamma=$ ridge-to-groove ratio;

$k_{g}=$ number of grooves;

$e_{x}=$ dimensionless displacement in $X$-direction $\left(=E_{x} / \Delta R\right)$

$e_{y}=$ dimensionless displacement in $Y$-direction $\left(=E_{y} / \Delta R\right.$;

$e_{z}=$ dimensionless displacement in $Z$-direction $\left(=E_{z} / \Delta R\right)$;

boundary conditions: $P=0$ at $\Psi=\Psi_{\text {max }}=\pi / 2 ; P_{\text {cav }}=0$. 
In the computer program, which resembles very much the program of appendix III, the groove parameters can again be given in three regions, viz, from $\Psi_{\min }$ to $\Psi_{1}$, from $\Psi_{1}$ to $\Psi_{2}$ and from $\Psi_{2}$ to $\Psi_{\max }$.

The location of the centre of the sphere is measured from the concentric position and denoted by $E_{x}, E_{y}$ and $E_{z}$, viz. the displacements in respectively the $X-, Y$ - and $Z$-directions. The sphere rotates about the $Z$-axis in the positive sense with an angular velocity $\Omega$.

The calculation is based on the generalised Reynolds equation. Hence the influence of the so-called "end effects" (see chapter 5) is left out of account here.

The forces of reaction of the bearing in the $X-, Y$ - and $Z$-directions are denoted by $F_{x}, F_{y}$ and $F_{z}$, and the dimensionless equivalents by $f_{x}, f_{y}$ and $f_{z}$. Again it is true that

$$
f_{x}=\frac{F_{x}}{F_{0}}, \quad \text { etc. } ; \quad \text { and } \quad F_{0}=\frac{12 \eta \Omega R^{4}}{(\Delta R)^{2}}
$$

\subsubsection{Spherical spiral-groove bearing with optimum axial thrust (SOAT bearing)}

Muijderman ${ }^{7-3}$ ) determined that geometry of a spherical bearing which provided an optimum thrust in the concentric position (such a Spherical bearing with Optimum Axial Thrust we shall call a SOAT bearing). He considered a grooved sphere that worked, from its south pole to its equator, in conjunction with a smooth spherical cup. The grooves were also supposed to extend from south pole to equator. For an infinite number $\left(k_{g}=\infty\right)$ of rectangular grooves the optimum values of the parameters are:

$$
\alpha=16^{\circ}, \quad h_{s}=2 \cdot 6, \quad \gamma=1 .
$$

With the FEM it has become possible to calculate the reaction forces of such a bearing also in eccentric positions of the sphere. Results are given in fig. 7.21. Because, in practice, the grooves cannot begin at the south pole, the beginning of the grooves, $\Psi_{1}$, was fixed at $0.2 \mathrm{rad}$. The choice of $\Psi_{1}$ is not very critical and we found, for the concentric position, nearly the same result as Muijderman (sec. 7.3.5). The maximum axial load capacity occurs when the south pole touches the cup and is $2 \cdot 3$ times higher than the concentric load capacity. Figure $7.21 b$ shows that $f_{z}$ slightly depends on $e_{x}$ for the case that $e_{y}=e_{z}=0$. The attitude angle $\left(=\arctan \left(f_{y} \mid f_{x}\right)\right)$ of this bearing is small, about $45^{\circ}$, and this indicates good stability properties. Assuming a minimum allowable film thickness of $0.2 \times R$, we may state that the allowable radial load may be about $8 \%$ of the allowable axial load. 


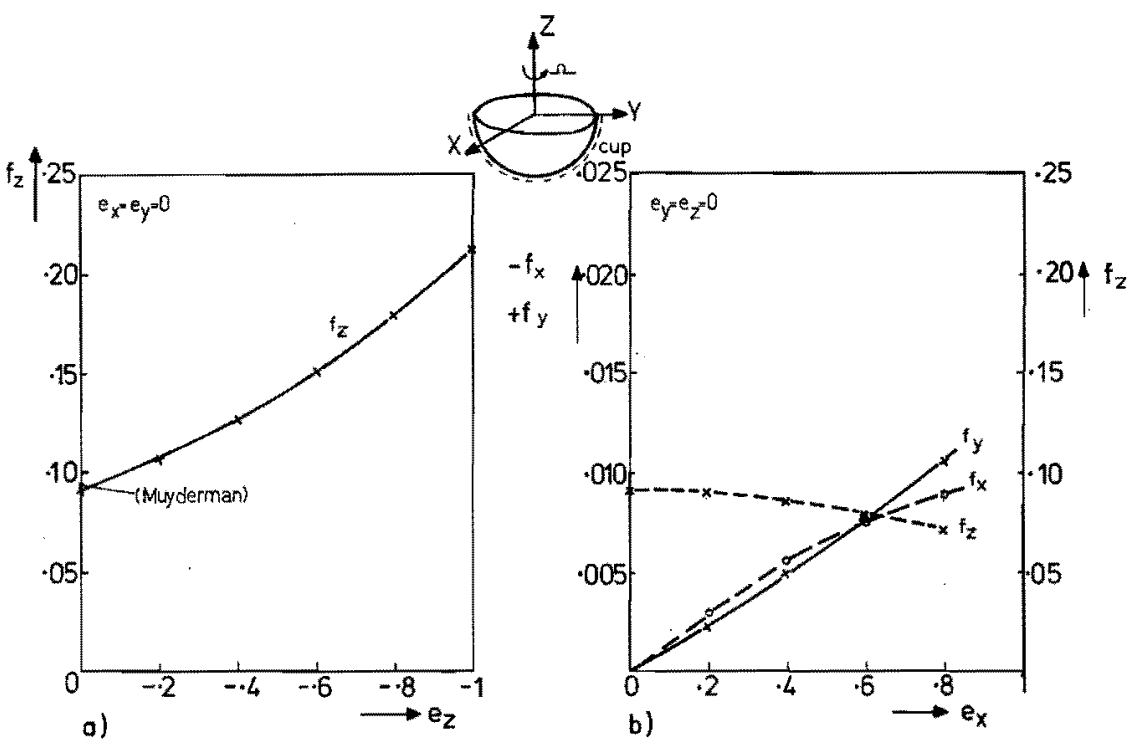

Fig. 7.21. Load capacity of a spherical bearing with optimum axial thrust (SOAT bearing); boundary conditions: $P=0$ for $\Psi=\pi / 2, P_{\text {cav }}=0$.

In fig. 7.22 the response coefficients are given as a function of the static deflection $e_{x}$, for $e_{z}$-values of $-0.5,0$ and +0.5 . In fig. 7.23 the stability curves for a symmetric system, with rigid rotor and rigidly supported SOAT bearings, are given. Regarding the response coefficients we may say that up to high values of the eccentricity $e_{x}$ the bearing behaves rotationally symmetrically, because

$$
\begin{aligned}
& a_{x x} \approx a_{y y}=a, \\
& b_{x x} \approx b_{y y}=b, \\
& a_{x y} \approx-a_{y x}=\delta_{c} b, \\
& \left.b_{x y} \approx-b_{y x}=b_{t} \quad \text { (see chapter } 3\right) .
\end{aligned}
$$

Moreover the response coefficients do not vary very much with $e_{x}$; this means that linearisation around the position $e_{x}=0$ is also permitted up to relatively large displacements.

If we approximate as follows:

$$
\begin{aligned}
a & =\frac{1}{2}\left(a_{x x}+a_{y y}\right), \\
b & =\frac{1}{2}\left(b_{x x}+b_{y y}\right), \\
\delta_{c} b & =\frac{1}{2}\left(a_{x y}-a_{y x}\right), \\
b_{t} & =\frac{1}{2}\left(b_{x y}-b_{y x}\right),
\end{aligned}
$$

then the theory for a rotationally symmetric bearing (chapter 3 ) gives almost the same results as those shown in fig. 7.23; for example, 
a..

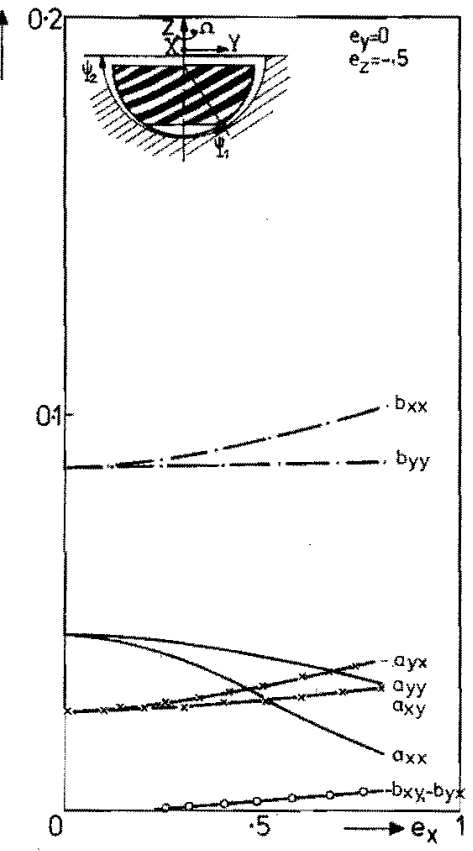

a)

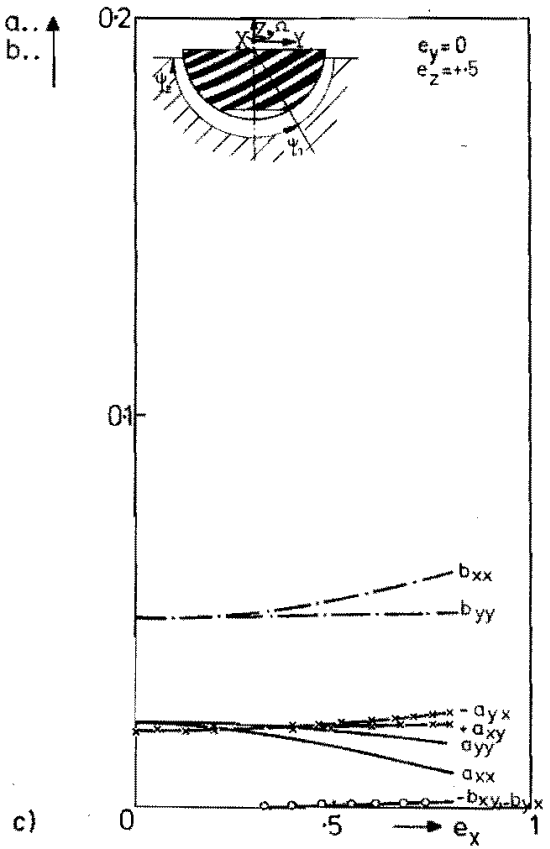

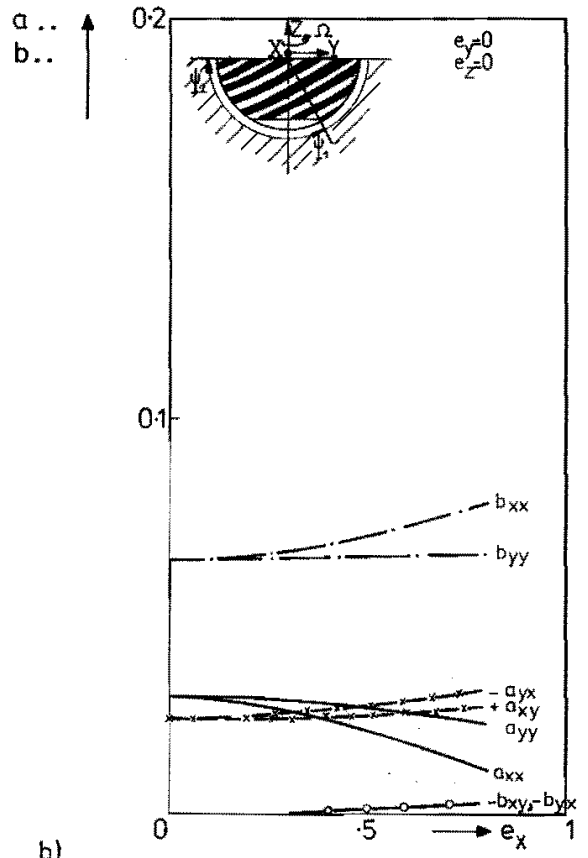

Fig. 7.22. Response coefficients of a SOAT bearing. (a): $e_{z}=-0.5 ;(b): e_{z}=0$; (c) $: e_{z}=0.5$. 

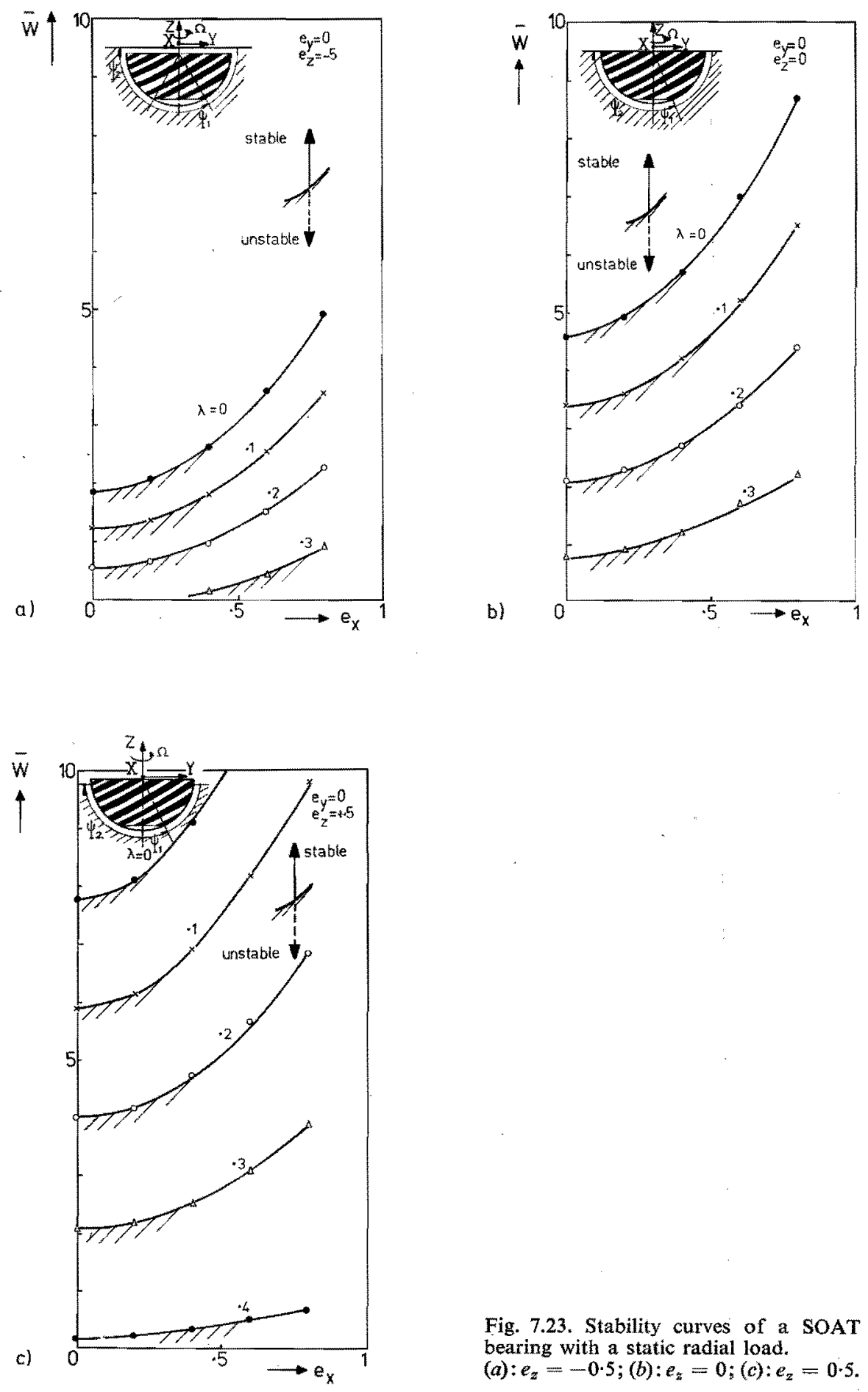

Fig. 7.23. Stability curves of a SOAT bearing with a static radial load.

$(a): e_{z}=-0.5 ;(b): e_{z}=0 ;(c): e_{z}=0.5$. 
(i) with $\lambda=e_{x}=e_{y}=e_{z}=0$ it follows from fig. 7.22 that $a=0.03 ; b=$ $0.064 ; \delta_{c} b=0.024 ; b_{t}=0$.

Application of (3.10) predicts a stability transition at $W=4.7$ (fig. 7.23 indicates $\bar{W}=4 \cdot 6$ );

(ii) with $\lambda=0.2$ and $e_{x}=e_{y}=e_{z}=0$ we obtain $\bar{W}=2.2$ (fig. 7.23 indicates $\bar{W}=2 \cdot 1)$;

(iii) with $\lambda=e_{y}=e_{z}=0, e_{x}=0.8: a=0.018 ; b=0.072 ; \delta_{c} b=0.029$; $b_{t}=0 ; \bar{W}=8.9$ (fig. 7.23 indicates $\bar{W}=8.7$ ).

We may conclude that, for practical applications, the assumption of a rotationally symmetric response gives sufficiently accurate results. If the same assumption is used in more-complex systems, for instance, with flexible rotors or flexible supports, the analysis is also considerably simplified.

\subsubsection{Spherical spiral-groove bearing with optimum radial stiffness (SORS bearing)}

In some applications of spherical bearings the axial load is not crucial, but it is precisely the radial load capacity which is inadequate. The FEM offers the possibility to optimise a bearing for certain characteristics. This quality of the FEM was used to determine the parameters for which the radial stiffness $\left(a_{x x}\right)$ in the concentric position was optimum. We shall call such a Spherical bearing with Optimum Radial Stiffness a SORS bearing.

For a bearing, in which the lubricant film extends from the south pole to the equator and the grooves from $\Psi_{1}=0.2 \mathrm{rad}$ to the equator, the optimum groove parameters for an infinite number of rectangular grooves $\left(k_{g}=\infty\right)$ are:

$$
\alpha=37^{\circ}, \quad h_{s}=1 \cdot 1, \quad \gamma=1 .
$$

Figure 7.24 gives the load capacity as a function of $e_{z}\left(e_{x}=e_{y}=0\right)$ and as a function of $e_{x}\left(e_{y}=e_{z}=0\right)$. It transpires that this bearing is in nearly every respect superior to the SOAT bearing: the radial load capacity is about three times higher and the axial load capacity for $e_{z}=-1$, is $20 \%$ higher. With an allowable film thickness of $0.2 \times R$ the allowable radial load is about $25 \%$ of the allowable axial load ( $8 \%$ for the SOAT bearing).

In fig. 7.25 the response coefficients are plotted versus $e_{x}$. At higher values of $e_{x}$ the coefficients are more "asymmetric" than those of the SOAT bearing. This is attributable to the fact that for this bearing and under the conditions used $\left(P_{\text {cay }}=0\right)$ cavitation sets in at $e_{x}>$ ca 0.4 .

Figure 7.26 shows the stability curves of a symmetrical rotor-and-bearing system with SORS bearings for the case that both the rotor and the bearing supports are rigid (cf. chapter 2). It may be surprising that, in spite of the higher radial stiffness of the SORS bearing, the stability at $e_{x}=0$ is only slightly better than that of the SOAT bearing. This can be accounted for by 

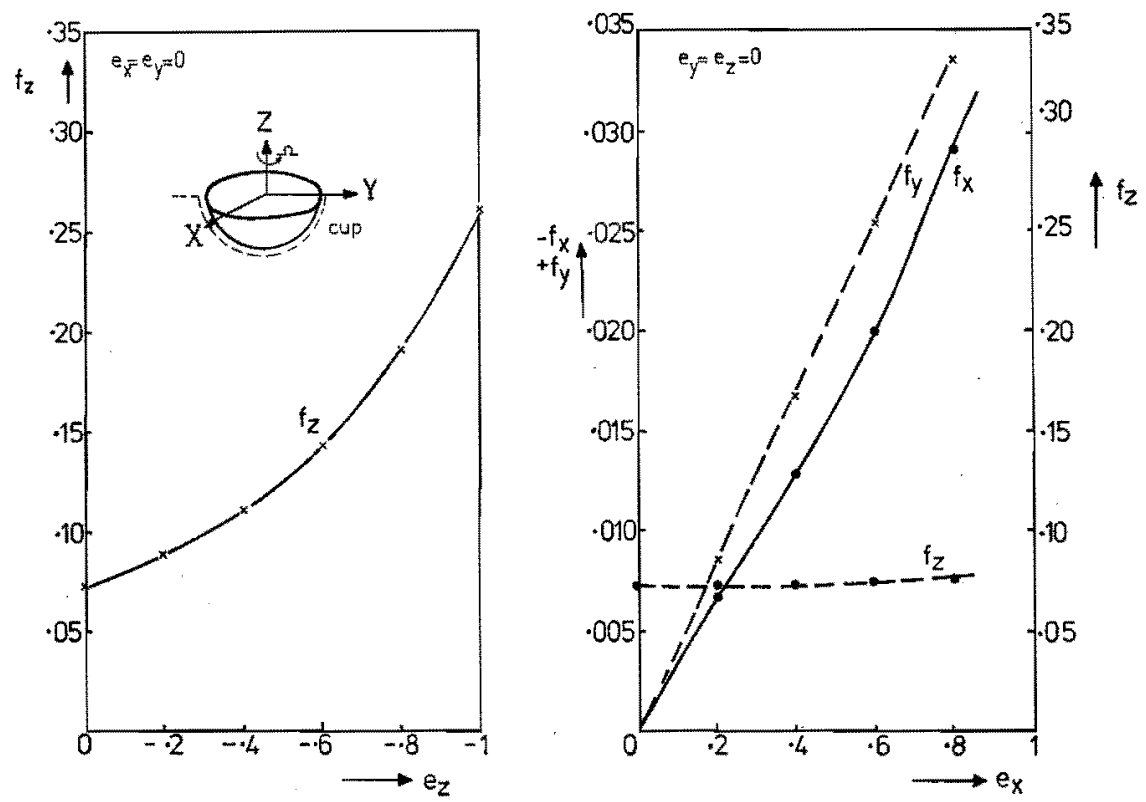

Fig. 7.24. Load capacity of a spherical bearing with optimum radial stiffness (SORS bearing); boundary conditions: $P=0$ for $\Psi=\Psi_{2}=\pi / 2, P_{\text {cav }}=0$.

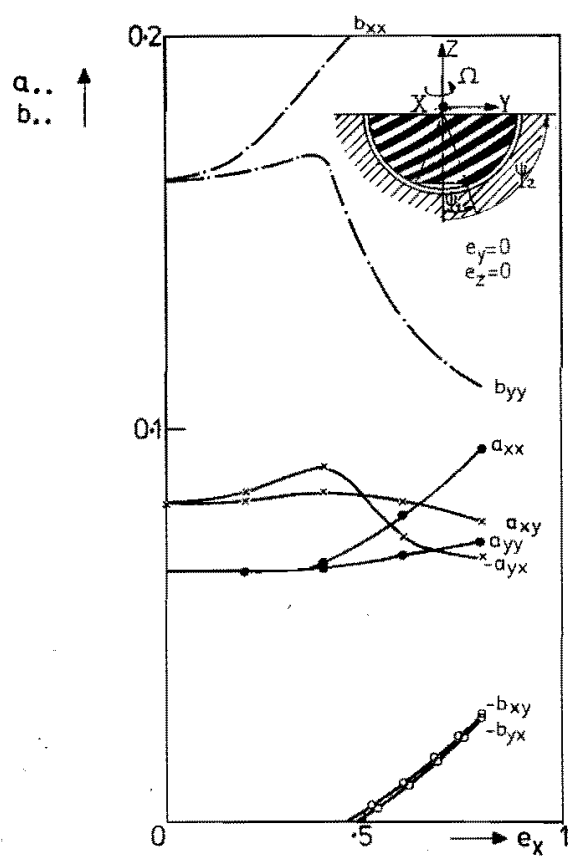

Fig. 7.25, Response coefficients of a SORS bearing; boundary conditions: $P=0$ for $\Psi=$ $\Psi_{2}=\pi / 2, P_{\mathrm{cav}}=0$.

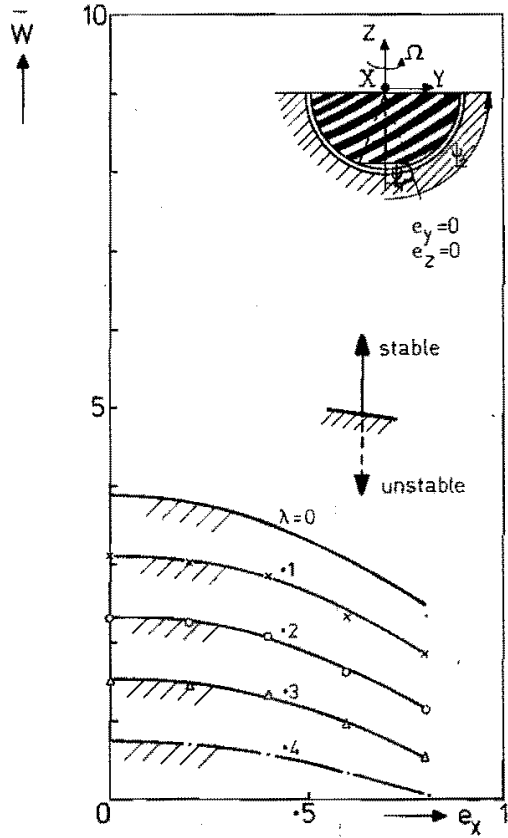

Fig. 7.26. Stability curves of a SORS bearing with a static radial load. 
the fact that the characteristic whirl frequency, $\delta_{c}$, is much higher for this bearing, which appears from the following example.

\section{Example}

With $\lambda=e_{x}=e_{y}=e_{z}=0$ it follows from fig. 7.25 that $\delta_{c} b=0.081$, $b=0.163$; this gives $\delta_{c}=0.50$ (SORS bearing), while from fig. $7.22, \delta_{c} b=$ $0.024, b=0.064$ and $\delta_{c}=0.37$ (SOAT bearing).

The fact that in (3.10) $\delta_{c}$ occurs in a quadratic form has such a strong effect that, if the stability is considered, the high stiffness of the SORS bearing is almost compensated by the high characteristic whirl frequency, $\delta_{c}$. This makes it clear that, if instead of the radial stiffness, the stability is to be optimum, then parameters such as $\delta_{c}$ should be considered also.

If in the same way as in the preceding section the theory of rotationally symmetric bearing response is used for the SORS bearing, stability data can be calculated that are sufficiently accurate for practical purposes.

Example

With $\lambda=e_{y}=e_{z}=0, e_{x}=0.8$ we find:

$$
\begin{aligned}
a & =\frac{1}{2}\left(a_{x x}+a_{y y}\right)=0.084, \\
b & =\frac{1}{2}\left(b_{x x}+b_{y y}\right)=0.174, \\
\delta_{c} b & =\frac{1}{2}\left(a_{x y}-a_{y x}\right)=0.072, \\
b_{t}=\frac{1}{2}\left(b_{x y}-b_{y x}\right) & =0
\end{aligned}
$$

and (3.10) predicts a stability transition at

$$
\widetilde{W}=\frac{\delta_{c}^{2}\left(1-\lambda / \delta_{c}\right)}{a+\delta_{c} b_{t}}=2 \cdot 1
$$

With the theory of chapter 2 , where the asymmetry of the bearing response was taken into account, we found (fig. 7.26) $W=2 \cdot 5$. In practical applications such a difference will as a rule be negligible.

\section{REFERENCES}

7-1) O. Pinkus and B. Sternlicht, Theory of hydrodynamic lubrication, McGraw-Hill Book Co., Inc., New York, 1961.

${ }^{7-2}$ ) H. Sassenfeld and A. Walther, Gleitlagerberechnungen, VDI-Forschungsheft 441, edition B, vol. $20,1954$.

7-3) E. A. Muijderman, Spiral-groove bearings, Philips Res. Repts Suppl. 1964, No. 2.

7-4) C. Y. Chow and J. H. Vohr, Helical-groove journal bearing operated in turbulent regime, ASME-ASLE Lub. Conference, Houston, Texas (Oct. 1969), Paper No. 69-Lub. 28. 


\section{DISCUSSION, CONCLUSIONS AND RECOMMENDATIONS OF FURTHER RESEARCH}

\subsection{Discussion}

In the previous chapters a number of problems concerned with the dynamics of rotor-and-bearing systems, particularly in respect of the stability, have been considered. On the one hand the results obtained can be applied directly to the design of rotor-and-bearing systems, and on the other hand the knowledge acquired opens new avenues of further research and development of such systems.

The work described in this report has been concerned mainly with rotors having sliding bearings, principally because of the good prospects which these bearings offer in the realising of high-speed rotors. Since the mastery of the dynamics in the application of such bearings resolves itself mainly into mastery of the stability, it is this aspect which has been treated in greater detail.

Excepting the general introduction and the present chapter, this work can be divided into three parts. In the first part, chapters 2,3 and 4, the dynamics and particularly the stability of a number of rotor-and-bearing systems have been examined, the assumption being made, for the moment, that the sliding bearings used are elements with familiar properties.

In the second part, chapters 5 and 6 , a technique, the finite-element method (FEM), has been developed for the calculation of the data of the "slidingbearing" element which are needed for the rotor dynamics of the first part.

In the third part, chapter 7 , the results have been presented of calculations by the finite-element method of a number of important types of bearings. Furthermore, inter alia by means of the theory in chapter 2, an indication has been given of the "stability" of those bearings.

The rotor-and-bearing systems that have been treated are assumed to be "symmetrical". A symmetrical system is here taken to mean

(a) that the rotor is rigid as well as rotationally symmetric;

(b) that the entire system, viz. rotor, bearing, bearing support, and external load, is symmetrical with respect to a plane perpendicular to the axis of rotation (symmetrical with respect to aa' in fig. 2.1).

These specifications of the symmetry do represent a limitation, but they clearly show also the influence of an effect such as the gyroscopic effect.

\subsubsection{Outline of chapters $2-7$}

Let us now turn to a brief discussion of chapters 2 to 7 .

\section{Chapter 2}

In practice we often find that both the rotor and the bearing supports may 
be considered to be rigid. Here these conditions have been examined in respect to a "symmetrical system" with sliding bearings.

In the first part of chapter 2 we have assumed for our starting point that the external load is static, so that the two bearings will have equal static deflections in the equilibrium position. Unlike the approach familiar from the literature, gyroscopic effects have been taken into account in our considerations of the stability. It turns out that there are two modes of motion, and that the stabilities associated therewith can be represented in a single diagram. An important point is that gyroscopic effects occur only in the "conical" mode and then they have mostly a stabilising influence; in the case of rotors with a large polar moment of inertia with respect to the axis of rotation, instabilities in this mode can even be suppressed completely. A stability diagram has been constructed for rotors with plain journal bearings; this makes it possible to calculate whether such a rotor will be stable or unstable.

In the second part of the chapter the corresponding case, in which the load of the rotor is not stationary but rotates in synchronism with the rotor, has been considered. In the equilibrium position the centres of both journals then describe circles around the centres of the bearing bushings. Such a loading condition occurs if, for example, a vertical rotor in plain journal bearings is loaded only by a force of unbalance. The conclusion was reached that an unbalance had a stabilising effect.

\section{Chapter 3}

In many cases it is justifiable to assume that the bearings used have a rotationally symmetrical response. Then it appears that the theory developed in chapter 2 can be simplified considerably and the stability of a symmetrical system with rigidly supported bearings can be described by a single equation (3.10).

Examples of bearings with a rotationally symmetrical response are, of course, rotationally symmetrical journal bearings, but unloaded bearings with three or more lobes come into this category, too. A number of grooved bearings, such as the helical-groove journal beating with optimum radial stiffness and the spherical bearing of chapter 7 have, to a first approximation, a rotationally symmetrical response, even with a "static" radial load in the resulting eccentric equilibrium position. In all these cases the stability can be determined quickly and easily by means of (3.10).

The simplification achieved on the assumption of rotationally symmetrical response makes it possible also to obtain an idea of the effect of "nonlinearities" in the bearing response, which begin to play a role in the case of big excursions from the equilibrium position of the bearing. Then it is found for an unloaded rotor, that in a case in which instability and unconstrained build-up of the excursions are predicted by the linear theory, the nonlinearities do in fact cause 
the excursions to remain limited, so that the bearing describes a "limit cycle" around the equilibrium position. For a rigid rotor with rigidly supported bearings, as that considered here, this means that the speed can be raised above the "linear stability limit" if the vibrations of the bearing due to the motion along the limit cycle are acceptable.

\section{Chapter 4}

Here the starting point is that the bearings of the rotor treated in the first part of chapter 2 are supported not rigidly but flexibly. Such a support can consist of "springs" and "dampers", but also of a second bearing (floatingbush bearing).

In the first part of this chapter a support with springs and dampers has been examined. Stability diagrams have been calculated for a few important types of journal bearings. These stability diagrams (figs $4.5,4.7$ and 4.8 ) have a characteristic form:

for a given eccentricity of the bearing and a given rigidity of the support the stability with low damping of the support (which means that for a given rotor the instability region starts at lower speeds) is poorer than in the case of a rigid support; an increase in the damping leads in the first instance to an improvement in the stability, and in conjunction with sufficiently low rigidity the support can make the rotor inherently stable, i.e. stable at all speeds; at very high values of the damping the support behaves as if it were completely rigid.

When designing high-speed rotors in particular, the inherently stable region in the stability diagrams appears to be of the utmost importance. Since an appropriate choice of the support parameters can be critical, an analysis like that presented here is indispensable.

In the second part of the chapter the "floating-bush bearing" has been treated. It was known already that these bearings in their existing forms possessed good stability. From the stability theory presented there, a new insight is obtained into the design of a floating-bush bearing with optimum stability; this may lead to new designs which will depart considerably from current conceptions.

\section{Chapter 5}

This chapter has laid the basis of the Finite Element Method for calculating the pressure variations in liquid-lubricated sliding bearings. FEM is familiar from mechanics and finds ever wider application, among other things for the calculation of stresses. Here the method is used for the calculation of the flow of a "Newtonian liquid" in a lubricant film. The starting point in the first part of the chapter is the "ordinary" differential equation of Reynolds, applicable to flow between smooth surfaces; in the second part a "generalised" Reynolds 
differential equation, applicable to flow between a grooved and a grooveless surface, is derived and then used in the FEM.

The method thus developed is of such general applicability that, provided that the lubricant film is completely filled with lubricant (cavitation being treated separately in chapter 6), almost all types of sliding bearings can be calculated with one kind of computer program.

In the present work the method is used only for those data of grooved and grooveless cylindrical bearings and of grooved spherical thrust bearings which are needed for an analysis of the stability, but it is equally possible to calculate the load capacity, attitude angle, response coefficients, etc., of other kinds of bearings such as conical bearings, spiral-groove thrust bearings, and lobed bearings. The flexibility of the method in respect to the calculation of the effects of geometrical changes in a bearing makes the method an important aid in the design of new types and shapes of bearings.

\section{Chapter 6}

This chapter forms an extension of chapter 5 in the sense that the method of calculation given in chapter 5 has been supplemented to cover bearings which, because of the occurrence of cavitation, are not filled completely by lubricant. After all, the phenomenon of cavitation does occur frequently in liquid bearings, and the examples given here show that it may have an important influence on the load capacity and the response coefficients.

\section{Chapter 7}

In this chapter the accuracy of the method of calculation developed in chapters 5 and 6 has been checked by comparison with, among other things, exact results, and the data required for the stability calculation of several types of bearings have been determined. In addition, after the response of a bearing has been determined, the stability of a symmetric rotor-and-bearing system in which such bearings find application has been calculated by means to the theory of chapter 2. The following types of bearings have been treated in turn:

- plain bearing with axial lubricant groove (ALG bearing);

- plain bearing with circumferential lubricant supply (CLG bearing);

- helical-groove bearing with optimum radial stiffness (ORS bearing);

- grooved spherical bearing with optimum axial thrust (SOAT bearing);

- grooved spherical bearing with optimum radial stiffness (SORS bearing).

The fact that spherical bearings can be calculated at all forms an illustration of the potential of the method with respect to the calculation of bearings with a complicated geometry. An example of a case in which FEM has been used for the design of a bearing with certain desired characteristics (for example optimum radial stiffness) is the SORS bearing. 


\subsubsection{Conclusions}

On the basis of the above, the following conclusions appear justifiable:

(1) The finite-element method (chapter 5) and its extension to bearings subject to cavitation (chapter 6) yield a unified approach for calculating all types of sliding bearings lubricated by a Newtonian liquid, with almost the same numerical procedure. Moreover, the ease with which the effect of geometrical modifications of a bearing can be calculated makes it possible to develop new types of bearings that have certain desirable characteristics.

(2) The stability of "symmetrical" rotor-and-bearing systems with rigid rotor and with rigidly supported bearings can be determined by means of the theory of chapter 2; whenever the reaction of the bearing may in addition be assumed to be rotationally symmetrical, it becomes possible to use the simple theory of chapter 3 and thus to take into account nonlinear effects.

(3) The stability of "symmetrical" rotor-and-bearing systems having rigid rotors and flexibly supported bearings can be determined by means of the theory given in chapter 4.

(4) Chapter 7 contains basic data of several familiar types of self-acting bearings which are useful for stability calculations.

\subsection{Final remarks}

\subsubsection{Flexible rotors}

In the above it has invariably been assumed that the rotor is rigid, in other words that the distortions of the rotor are negligible.

For the theoretically more complicated "symmetrical" rotor-and-bearing systems in which the rotor is flexible it is possible to utilize the fact that at the boundary between stability and instability a flexible rotor, with mass $M_{R}$, behaves in the translational mode as a rigid rotor of "apparent" mass $M_{R}{ }^{*}$. For $M_{R}{ }^{*}$, we then have

$$
M_{R}^{*}=\frac{M_{R}}{1-\left(\Omega_{w} / \Omega_{n t}\right)^{2}},
$$

in which $\Omega_{w}=$ angular frequency of whirl at the stability-instability boundary,

$\Omega_{n t}=$ angular frequency of the bending vibrations in the translational mode of a rotor rigidly supported at the position of the bearings.

If in the conical mode the gyroscopic effects may be neglected we obtain analogously:

$$
J^{*}=\frac{J}{1-\left(\Omega_{w} / \Omega_{n c}\right)^{2}},
$$


in which $J=$ moment of inertia of the rotor (taken perpendicularly to the axis of rotation and through the centre of gravity),

$J^{*}=$ apparent moment of inertia,

$\Omega_{n c}=$ angular frequency of bending vibrations in the conical mode.

The derivation of the above equations and the manner in which they can be used to obtain the data of flexible rotors from stability diagrams applicable to rigid rotors will not be considered further in this report.

\subsubsection{Asymmetric rotor}

The theories of chapters 2,3 and 4 have been developed for "symmetrical" rotors, so that it has become possible to resolve the motion of the system into two modes and to determine the stability of each mode separately. When such a resolution into modes is not permissible, a stability analysis becomes much more complicated, because a characteristic determinant twice as extensive has then to be examined.

In a number of cases it is possible to apply the theory of symmetric systems to asymmetric systems. An example, of fairly frequent occurrence, is the one in which the asymmetry of the rotor is compensated by the asymmetry of the bearing. Here we might consider the rotor of fig. 8.1 with centre of gravity $G$, mass $M_{R}$, moments of inertia $J$ and $I$, and rigidly supported bearings. If $F_{x 1}$ is the force acting on bearing 1 in the $x$-direction, $X_{1}$ the displacement of the journal, etc., then the equations of motion are

$$
\begin{gathered}
F_{x 1}+F_{x 2}=M_{R}\left((1-\alpha) \frac{\mathrm{d}^{2} X_{1}}{\mathrm{~d} t^{2}}+\alpha \frac{\mathrm{d}^{2} X_{2}}{\mathrm{~d} t^{2}}\right), \\
F_{y 1}+F_{y 2}=M_{R}\left((1-\alpha) \frac{\mathrm{d}^{2} Y_{1}}{\mathrm{~d} t^{2}}+\alpha \frac{\mathrm{d}^{2} Y_{2}}{\mathrm{~d} t^{2}}\right) ; \\
-\alpha F_{x 1}+(1-\alpha) F_{x 2}=\frac{J}{L^{2}} \frac{\mathrm{d}^{2}\left(X_{2}-X_{1}\right)}{\mathrm{d} t^{2}}+\frac{I \Omega}{L^{2}} \frac{\mathrm{d}\left(Y_{2}-Y_{1}\right)}{\mathrm{d} t}, \\
-\alpha F_{y 1}+(1-\alpha) F_{y 2}=\frac{J}{L^{2}} \frac{\mathrm{d}^{2}\left(Y_{2}-Y_{1}\right)}{\mathrm{d} t^{2}}-\frac{I \Omega}{L^{2}} \frac{\mathrm{d}\left(X_{2}-X_{1}\right)}{\mathrm{d} t},
\end{gathered}
$$

Fig. 8.1. Asymmetric rotor-and-bearing system. 
where $\alpha=L_{1} / L_{2}$ (for $L_{1}$ and $L_{2}$, see fig. 8.1).

Provided that the following choice can be realised:

$$
\frac{A_{x x 1}}{A_{x x 2}}=\frac{A_{x y 1}}{A_{x y 2}}=\ldots=\frac{B_{x x 1}}{B_{x x 2}}=\ldots=\frac{1-\alpha}{\alpha},
$$

where $A_{x x 1}, \ldots, B_{x y 1}$ are the response coefficients of bearing 1 , and $A_{x x 2}, \ldots$, $B_{x y 2}$ those of bearing 2, such "asymmetric" bearings compensate the asymmetry of the rotor and then the motion can still be resolved into a translational mode and a conical mode. The former mode is described by (8.3), in which $X_{1}=X_{2}$, and the latter by (8.4), with $\alpha X_{2}=-(1-\alpha) X_{1}$. The analysis of either mode can then be performed separately by means of the theory of chapter 2.

Even in cases in which (8.5) holds only approximately, a resolving into two modes often leads to a result which is still sufficiently accurate for design purposes. Relations of the form of (8.5) are encountered more or less as a matter of course whenever, on account of a gravitational load, bearing 1 of the rotor shown in fig. 8.1 must in any case be given a heavier construction than bearing 2.

\subsubsection{Experimental verification}

Many of the results of chapters 2 to 7 can be checked by means of data known from the literature. Thus the results of load-capacity calculations by means of FEM, among others those in chapter 7 , have been compared with data obtained previously by others by different methods, the correctness of which had already been established. Thus the response coefficients of smooth bearings calculated by means of FEM (cf. figs 2.3 and 2.4) are in good agreement with data from the literature if allowance is made for the various ways in which cavitation is taken into account. Furthermore the stability curve of the translational mode in fig. 2.5 differs but little from the results of Holmes ${ }^{8-2}$ ).

Even though experience with rotor-and-bearing systems (flywheel for space vehicles ${ }^{8-3}$ ), experimental gyroscopes, etc.) does confirm the theory, experimental verification of a number of results is still desirable. In particular the stability of rotors with flexible supports (chapter 4) should be investigated experimentally, above all because of the great importance of such designs in numerous applications. Such experiments, though they are being prepared to fill in these gaps, will not be treated in further detail within the scope of the present report.

\subsubsection{Computer programme for transforming the characteristic determinant into the characteristic equation}

A serious objection to the Routh-Hurwitz method, frequently used in this work for stability analyses, has always been that the "characteristic determi- 
nant" had to be converted into the "characteristic equation" (cf. chapter 1). As the number of parameters describing the motion of a system increases, the order of the determinant becomes greater, and if the characteristic equation has to be found by manual multiplication, insuperable difficulties soon arise.

This has now changed because lately computer programs have become available by means of which algebraic operations can be performed on a computer (e.g. FORMAC-IBM). The forming of the characteristic equation can then be programmed and, even with big systems, no longer presents a problem.

\subsection{Possibilities of further investigations and development}

\subsubsection{Extension of the above theories}

Obviously it is good sense to extend the above theories which have proved to be a reliable basis, in the direction of systems of greater complexity. In particular those systems of which the asymmetry precludes a resolution into modes of motion ought to be investigated in more detail. Further analysis is desirable also in respect of the behaviour of flexible rotors, particularly for high-speed applications.

Partly on account of the fact that the characteristic determinant can now be transformed easily into the characteristic equation (cf. previous remarks) the analysis of more-complicated systems can be performed with the same techniques as used above, and this will not give rise to any novel difficulties. The distilling and generation of regular patterns, such as the existence of an optimum damping of the bearing support in the cases dealt with in chapter 4 , is here of the greatest importance.

\subsubsection{New forms of sliding bearings and of rotor-and-bearing systems}

The better insight into the dynamics, and particularly into the stability, of conventional rotor-and-bearing systems, in conjunction with the better possibilities of calculation, may well prove helpful in creating novel designs of highspeed rotor-and-bearing systems. Thus chapter 4 shows the potentialities of a flexible bearing support, and a few possible designs have already been mentioned in that chapter. Further investigation into the potentialities of bearing supports appears to be desirable, however, and may yield worthwhile rewards.

In rotor-and-bearing systems for high speeds it is necessary to keep the dissipation of energy in the (sliding) bearings as low as possible. Since the principal dimensions of a bearing are usually determined by factors external to the bearing (e.g. resonance frequencies of the rotor), one is often forced, in the case of the conventional forms of sliding bearings, to choose a lubricant with very low viscosity, for example a gas, so as to obtain a low dissipation for a certain load capacity. This does not, however, lead to bearings of very high efficiency, i.e. those in which the specified load capacity or stiffness are obtained with the 
least possible dissipation. Since it is the application of a highly viscous lubricant, in particular, that can lead to efficient bearings it becomes desirable, by means of the theories of chapters 5 and 6 , to develop new bearing geometries which, for specified principal dimensions and lubricants, such as oil, do prove suitable for high speeds.

FEM can therefore be used here, as has already been done in the case of the spherical bearing with optimum radial stiffness (SORS bearing), to find bearing geometries that have the desirable characteristics.

\section{REFERENCES}

${ }^{8-1}$ ) J. W. Lund, The stability of an elastic rotor in journal bearings with flexible, damped supports, J. appl. Mech., Trans. ASME 32, 911-920, 1965.

8-2) R. Holmes, Instability phenomena due to circular bearing oil films, J. mech. Eng. 8. $419-425,1966$.

8-3) J. P. Reinhoudt, A flywheel for stabilizing space vehicles, Philips tech. Rev. 30, 2-6, 1969. 


\section{APPENDIX I}

From (2.15) it follows that the characteristic determinant and the characteristic equation for the translation of a rigid symmetric rotor with rigid bearing supports are given by

$$
\begin{gathered}
\left|\begin{array}{rr}
s^{2}+\bar{W}\left(b_{x x} s+a_{x x}\right) & W\left(b_{x y} s+a_{x y}\right) \\
\bar{W}\left(b_{y x} s+a_{y x}\right) & s^{2}+W\left(b_{y y} s+a_{y y}\right)
\end{array}\right|=0, \\
a_{0} s^{4}+a_{1} s^{3}+a_{2} s^{2}+a_{3} s+a_{4}=0 .
\end{gathered}
$$

In the so-called zero-point method it is assumed that, at the stability-instability transition, there is a pair of purely imaginary roots for $s$. These roots can be written as

$$
s=\mathrm{j} \omega \quad(\omega \text { real })
$$

The equation (AI.1) can be divided into

$$
\begin{gathered}
a_{0} \omega^{4}-a_{2} \omega^{2}+a_{4}=0, \\
\omega\left(a_{1} \omega^{2}-a_{3}\right)=0 .
\end{gathered}
$$

If $a_{4} \neq 0$, then $\omega \neq 0$; in that case (AI.3) and (AI.4) lead to the equation for the value of $W$ at the stability-instability transition, $W_{\text {transition }}$ :

$$
a_{0} a_{3}^{2}-a_{1} a_{2} a_{3}+a_{4} a_{1}^{2}=0 .
$$

When $\bar{W}_{\text {transition }}$ is found from (AI.5), the two roots are defined by (AI.4) and the remaining pair of complex roots can be solved from (AI.2). Here, it should be emphasised that, for a transition from the stable state to the unstable state, these roots should have no positive real parts, otherwise there is no transition at all; the motion is unstable.

The results obtained with a rigid rotor having rigid bearing supports can be used to determine the stability of a symmetric rotor with a flexible shaft and/or a flexible bearing support if the shaft and support are rotationally symmetric and undamped.

According to (4.14) and (4.15) the characteristic determinant for the translation is:

$$
\left|\begin{array}{cccc}
s^{2}+\bar{W}\left(b_{x x} s+a_{x x}\right) & \bar{W}\left(b_{x y} s+a_{x y}\right) & s^{2} & 0 \\
\bar{W}\left(b_{y x} s+a_{y x}\right) & s^{2}+\bar{W}\left(b_{y y} s+a_{y y}\right) & 0 & s^{2} \\
s^{2} & 0 & s^{2}+\bar{W}^{2} k & 0 \\
0 & s^{2} & 0 & s^{2}+\bar{W}^{2} k
\end{array}\right|=0
$$


Here $k$ represents the total stiffness of shaft and support.

For $s^{2} \neq 0$,

$$
\begin{aligned}
& s^{2}+W^{2} k \neq 0, \\
& W^{2} k \neq 0,
\end{aligned}
$$

equation (AI.6) can be transformed into

with

$$
\left|\begin{array}{rr}
s^{2}+\bar{W}_{f}\left(b_{x x} s+a_{x x}\right) & \bar{W}_{f}\left(b_{x y} s+a_{x y}\right) \\
\bar{W}_{f}\left(b_{y x} s+a_{y x}\right) & s^{2}+\bar{W}_{f}\left(b_{y y} s+a_{y y}\right)
\end{array}\right|=0
$$

$$
W_{f}=\left(\frac{s^{2}}{W^{2} k}+1\right) W
$$

Comparison of (AI.1) with (AI.7) reveals that both equations have the same pairs of purely imaginary roots at the transition points, and

$$
W_{\text {transition }}\left(\text { from (AI.1)) }=\bar{W}_{f \text { transition }}(\text { from (AI.7)). }\right.
$$

If $s_{1}, s_{2}, s_{3}$ and $s_{4}$ are the roots of (AI.1) and $s_{1}{ }^{*}, s_{2}{ }^{*}, s_{3}{ }^{*}, s_{4}{ }^{*}, s_{5}{ }^{*}$ and $s_{6}{ }^{*}$ those of (AI.7), we may put, at a transition point:

$$
\begin{aligned}
& s_{1}=s_{1}^{*}, \quad \text { the imaginary roots } \\
& s_{2}=s_{2}{ }^{*} .
\end{aligned}
$$

Also $s_{3}{ }^{*}, s_{4}{ }^{*}$ lie in the same half-plane as $s_{3}, s_{4}$ because

$$
\begin{aligned}
& \lim _{k \rightarrow \infty} s_{3}{ }^{*}=s_{3}, \\
& \lim _{k \rightarrow \infty} s_{4}^{*}=s_{4},
\end{aligned}
$$

and $s_{3}{ }^{*}, s_{4}{ }^{*}$ remain, for finite values of $k$, in the same half of the complex plane; otherwise (AI.7) would have more transition points than (AI.1).

What about the pair $s_{5}{ }^{*}, s_{6}{ }^{*}$ ? We shall now see that, for "normal" sliding bearings, and large values of $k$ this pair has negative real parts (as should be the case for a transition). If this can be demonstrated they remain, just as $s_{3}{ }^{*}, s_{4}{ }^{*}$, in the left-hand half of the complex plane.

From (AI.6) we obtain:

$$
\begin{aligned}
& \frac{s^{6}}{(k \bar{W})^{2}}\left(b_{x x} b_{y y}-b_{x y} b_{y x}\right)+ \\
+ & \frac{s^{5}}{k \bar{W}}\left(b_{x x}+b_{y y}+\frac{1}{k \bar{W}}\left(b_{x x} a_{y y} \ldots\right)\right)+ \\
+ & s^{4} \quad\left(1+\frac{1}{k \bar{W}}\left(b_{x x} b_{y y} W \ldots\right)+\frac{1}{(k \bar{W})^{2}}\left(a_{x x} a_{y y} \ldots\right)\right)+ \\
+ & s^{3} \quad\left(\bar{W} b_{y y}+W b_{x x}+\frac{1}{k \bar{W}}(\ldots)\right)+
\end{aligned}
$$




$$
\begin{array}{ll}
+s^{2} & \left(\bar{W} a_{y y}+\bar{W} a_{x x}+\bar{W}^{2} b_{x x} b_{y y}-\bar{W}^{2} b_{x y} b_{y x}+\frac{1}{k W}(\ldots)\right)+ \\
+s \quad & \left(\bar{W} b_{x x} a_{y y} \ldots\right)+ \\
+\quad & \left(\bar{W}^{2} a_{x x} a_{y y}-W^{2} a_{x y} a_{y x}\right)=0 .
\end{array}
$$

As $k$ tends to infinity the characteristic equation (AI.12) reduces to the characteristic equation (AI.2) and the roots $s_{5}{ }^{*}$ and $s_{6}{ }^{*}$ tend to infinity; these roots can be found from

$$
\left(\frac{s}{k W_{\mathrm{tr}}}\right)^{2}\left(b_{x x} b_{y y}-b_{x y} b_{y x}\right)+\left(\frac{s}{k W_{\mathrm{tr}}}\right)\left(b_{x x}+b_{y y}\right)+1=0 .
$$

With

$$
\begin{aligned}
& \alpha_{1}=b_{x x} b_{y y}-b_{x y} b_{y x}, \\
& \alpha_{2}=b_{x x}+b_{y y},
\end{aligned}
$$

and

$$
b_{x x}>0, \quad b_{y y}>0, \quad b_{x x} b_{y y} \gg b_{x y} b_{y x}
$$

for "normal" sliding bearings, it is true that

$$
\alpha_{1}>0, \quad \alpha_{2}>0
$$

Further

$$
\left(\frac{s_{5,6^{*}}}{k \bar{W}_{\mathrm{tr}}}\right)=\frac{-\alpha_{2} \pm\left(\alpha_{2}^{2}-4 \alpha_{1}\right)^{1 / 2}}{2 \alpha_{1}} .
$$

(AI.14) and (AI.15) imply that $s_{5}{ }^{*}, s_{6}{ }^{*}$ have negative real parts, which was to be demonstrated. 
ARFEADIX II Coefflcienta of the Characteristic Equation

$a_{0}=-b 43 \times b 34-b 21 \times b 34+b 33 \times b 44+b 11 \times b 44+b 22 \times b 33-b 12 \times b 43-b 12 \times b 21+b 11 \times b 22$

$a_{1}=-b 22 \times b 43 \times b 34-b 11 \times b 43 \times 634-c 43 \times 034-c 27 \times b 34-b 43 \times c 34-b 21 \times c 34+b 22 \times b 33 \times b 44$ b11 $\times b 33 \times 044+c 33 \times 144-b 21 \times b 12 \times b 44+b 11 \times b 22 \times b 44+c 11 \times b 44+b 33 \times c 44+$ b11 $1 \times 44-b 21 \times b 12 \times b 33+b 11 \times b 22 \times b 33+c 22 \times b 33+b 22 \times c 33-c 12 \times b 43-b 12 \times c 43-$ $c 21 \times b 12-b 21 \times c 12+c 11 \times b 22+b 11 \times c 22$

$a_{2}=+b 21 \times 612 \times b 43 \times b 34-b 11 \times b 22 \times b 43 \times b 34-c 22 \times b 43 \times b 34-c 11 \times b 43 \times b 34-b 22 \times 043 \times b 34$

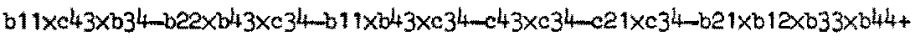

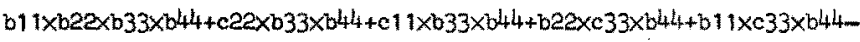
$c 21 \times b 12 \times b 44-b 21 \times c 12 \times b 44+c 11 \times b 22 \times b 44+b 11 \times c 22 \times b 44+b 22 \times b 33 \times c 44+$

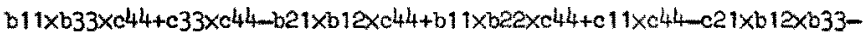
b21 $\times 112 \times b 33+c 11 \times b 22 \times b 33+b 11 \times c 22 \times b 33-b 21 \times b 12 \times c 33+b 11 \times b 22 \times c 33+$

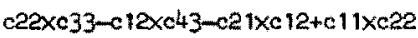

$a_{3}=+c 21 \times b 12 \times b 43 \times b 34+b 21 \times c 12 \times 643 \times b 34-c 11 \times b 22 \times b 43 \times b 34-b 11 \times c 22 \times b 43 \times b 34+$ b21 $1 \times 12 \times c 43 \times b 34-b 11 \times b 22 \times c 43 \times b 34-22 \times c 43 \times b 34-017 \times c 43 \times 034+b 21 \times b 12 \times 043 \times c 34$

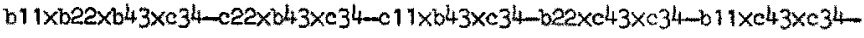

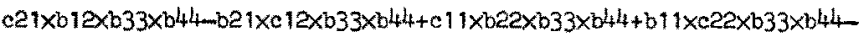
b2 $1 \times b 12 \times c 33 \times b 44+b 11 \times b 22 \times c 33 \times b 44+c 22 \times c 33 \times b 44+c 11 \times c 33 \times b 44-c 21 \times c 12 \times b 44+$

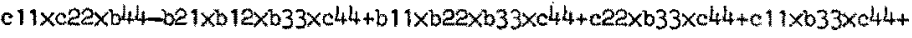

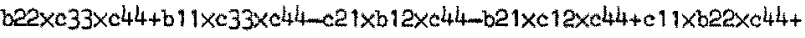

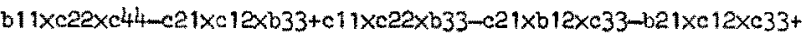
$\mathrm{c} 11 \times \mathrm{b} 22 \times \mathrm{c} 33+\mathrm{b} 11 \times \mathrm{c} 2 \mathrm{2} \times \mathrm{c} 33$

$a_{4}=+c 21 \times 012 \times b 34 \times b 43-c 11 \times c 22 \times b 43 \times b 34+c 21 \times 012 \times c 43 \times 034+b 21 \times c 12 \times c 43 \times 634-$

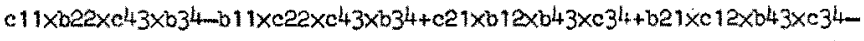
c11 $\times b 22 \times b 43 \times c 34-b 11 \times c 22 \times b 43 \times c 34+b 21 \times b 12 \times c 43 \times c 34-b 11 \times b 22 \times c 43 \times c 34$ $022 \times 043 \times c 34-11 \times c 43 \times c 34-c 21 \times c 12 \times b 33 \times b 44+c 11 \times c 22 \times b 33 \times b 44-c 21 \times b 12 \times c 33 \times b 44$ b2 $1 \times c 12 \times c 33 \times b 44+c 11 \times b 22 \times 033 \times b 44+b 11 \times c 22 \times c 33 \times 044-c 21 \times b 12 \times b 33 \times c 44-$ b21 $\times c 12 \times b 33 \times c 44+c 11 \times b 22 \times b 33 \times c 44+b 11 \times c 22 \times b 33 \times c 44-b 21 \times b 12 \times c 33 \times c 44=$

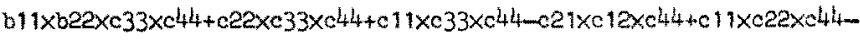
$c 21 \times c 12 \times c 33+c 11 \times c 22 \times c 33$

$a_{5}=+c 21 \times c 12 \times c 43 \times b 34 c 11 \times c 22 \times c 43 \times b 34+c 21 \times c 12 \times b 43 \times c 3^{4}-11 \times c 22 \times b 43 \times c 34$. $c 21 \times b 12 \times c 43 \times c 34+b 21 \times c 12 \times c 43 \times c 3^{4}-c 11 \times b 22 \times c 43 \times c 34-b 11 \times c 22 \times c 43 \times c 3^{4-}$ $c 21 \times c 12 \times c 33 \times b 44+c 11 \times c 2.2 \times c 33 \times b 44-c 21 \times c 12 \times b 33 \times c 44+c 11 \times c 22 \times b 33 \times c 44-$ $c 21 \times b 12 \times c 33 \times c 44-021 \times c 12 \times c 33 \times c 44+c 11 \times 022 \times c 33 \times c 44+b 11 \times c 22 \times c 33 \times c 44$

$a_{6}=+c 21 \times 012 \times c 43 \times c 3^{4}-c 11 \times c 22 \times c 43 \times c 3^{4}-c 21 \times 012 \times 033 \times c 44+c 11 \times c 22 \times c 33 \times c 44$ 


\section{APFENDIX III Computer Progran for a Cylindrical Journal Bearing}

\section{begin comment ip reinhoudt, box 57}

berekening clilnarisch glijlager met elementen methode,

en alcemene vrgl van reynolds.

de berekening van de matrix en de krachten is gelineatriseerd 22-1-71;

Intexer n, nloop, nkmex, prkn;

nkmax: $:=$ read; nloop: wread;

for n:=1 step 1 until nloop do

bestn

Ies] ex, ey, eptx, epty, eptz, om, mom, ho1, ho2, ho , ho, dri, dps, psimin, psi1, psic,

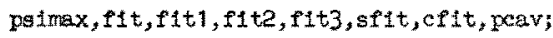

Integer $i, j, k, n, n k, m, s w 0, n f 1, n p s, n p s 1, n p s e, n p s 3, t ;$

armax pei, f1[1:nkmax];

procedure cholbd( $n, m$, dec, $a, b$, fail);

yalue $n, m$, dec; Integer $n, m$, dec; array $a, b$; label fail;

begin integer $k, \mathrm{ml}, 1, j$

$\mathrm{m} 1:=\mathrm{m}$

If $\mathrm{dec}=0 \vee \mathrm{dec}=2 \vee \mathrm{dec}=4 \vee \mathrm{dec}=6$ then

for $x:=1$ step 1 yutal $n$ do

begin if alk, 0$] \leq 0$ then goto fali;

$a[k, 0]:=\operatorname{sart}(a[k, 0])$;

If $\mathrm{m}>\mathrm{n}-\mathrm{k}$ then $\mathrm{m} 1: \mathrm{n}-\mathrm{k}$;

for $1:=1$ gtep 1 until $m$ do $a[k, 1]:=a[k, 1] / a[k, 0]$;

for $1:=m 1+1$ gtep 1 unt1 $m$ do $a[k, 1]:=0$

for $I:=1$ step 1 until $\mathrm{m} 1$ do

for $1:=0$ step 1 until $m 1-j$ do

$a[k+j, 1]:=a[k+j, 1]-a[k, j] \times a[k, j+1]$

end $k$;

$\mathrm{m} 1:=\mathrm{m}$;

1f dec $=0 \vee \mathrm{dec}=1 \vee \mathrm{dec}=4 \vee \mathrm{dec}=5$ then

for $k:=1$ step 1 until $n$ do

begs $b[k]:=b[k] / a[k, 0] ;$

If $m>n-k$ then $m:=n-k$;

for $f:=1$ step 1 until $\mathrm{ml}$ do

$b[k+j]:=b[k+j]-a[k, j] \times b[k]$

end $\mathrm{k}$;

$\mathrm{m} 1:=\mathrm{m}$;

11 dec $<4$ then

for $k:=n$ step -1 unt11 $1 \mathrm{dg}$

begin $b[k]:=b[k] / a[k, 0]$;

1 If $k<n+1$ then $m 1:=k-1$;

Lor $J:=1$ step 1 until n1 do

$b[k-j]:=b[k-j]-a[k-j, j] \times b[k]$

end $k$

end cholbd; 
mosedure schu1 $(1, j, k)$; Integer $1, j, k ;$ begtn latezer $s ; s:=1 ; 1:=j ; j:=k ; k:=8$ end ;

procedurs el $(n$, swo, wr); Integer $n$, swo; mocedure pr;

begin integer m;

for $m:=2$ atep 1 until nps do

begin 1: =npax $(n-1)+m$;

$j:=n p s \times(n+g w 0)+m ;$

$k:=\operatorname{mpsx}(n+s w 0)+m+1 ; t:=1 ; p r ;$

$1:=\operatorname{mpax}(n-1)+m$

$\mathrm{j}:=\mathrm{nps} \times(\mathrm{n}+\mathrm{sw})+\mathrm{m}+1$;

$\mathrm{k}:=\mathrm{nps} \times(n-1)+\mathrm{m}+1 ; t:=1 ; \mathrm{pr} ;$

end :

eng;

ex:=read; ey:=read; eptx:=read; epty;=read; eptz:=read; on:=read;

h01:=read; ho2:=read; ho3: mread;

nps 1:=read; npas:=read; nps3:=read;

ftt:=read; fit2;=read; $11+3:=$ read;

nf 1 : =read; paimin:=read; ps 1 : =read; pa12 : =read; ps 1 max : =read; gam: =read;

pcav : =read;

nlcr; printtext ( 4

ex ey eptx epty eptz om hol ho2 ho3 nps1 npse nps3 fit1 fite f1t

nf1 psimin ps11 psi2 psimax eam peavp);

nler; flxt $(0,1$, ex $) ; f 1 x t(0,1$, ey $) ; f 1 x t(0,2$, eptx $) ; f i x t(0,2$, epty); fixt $(0,2$, eptz);

f $1 x t(2,1,0 \mathrm{~m}) ; f \pm x t(2,1, \mathrm{no1}) ; f 1 \times t(2,1, h 02) ; f 1 \times t(2,1, h 03)$;

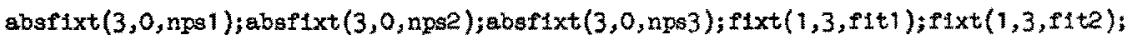

Plxt $(1,3, f 1+3) ; \operatorname{absf} 1 \times t(3,0, n f 1) ; 11 \times t(1,2$, psimin $) ;$

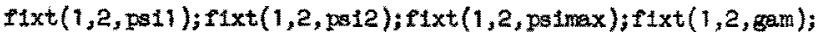

flot $(2,2$, pav $)$;

df1: =3.1416/nft;

fl[2]:=0; psi[2]:=psimin; swo:=1;

$n:=1 ;$

nps : $=n p s 1+n p a 2+n p s 3+1$;

$161:$

Lor $m:=2$ step 1 unt1l nps do

begin regl. test; test: $=p s i[(n-1) \times n p s+m]+m$;

$P 1[(n-1) \times n p s+m+1]:=11[(n-1) \times n p s+m]$;

If test<psil thep dps:=(ns11-psimin)/nps1

else if test<ps12 then dps:=(ps12-ps11)/npse

else If test<psimaxthen dps:=(na1max-ps12)/nps

else exit;

psi:[(n-1) $\times n p s+m+1]:=p s i[(n-1) \times n p s+m]+d p s$ end ;

$n: m+1 ;$

12 mexne1 then begin

$f 1[(n-1) \times n p s+2]:=f 1[(n-2) \times n p s+2]+s w 0 \times(n-1) \times d f 1 ;$

pal $[(n-1) \times n p s+2]:=p s 1[(n-2) \times n p s+2]$;

awo: $=-\$ w 0 ;$ soto $I g 1$ end; 
$m:=2 \times n p s+1 ; \quad n k:=2 \times n f l \times n p s+1 ; n l c r ; \operatorname{printtext}(\mathrm{knk}=1) ; f 1 \times t(3,0, n k)$;

begin grray a[ $1: n k, 0: m], v, p$, frac[1:nk]; rea] $f x, f y, f z$;

boolean srray randpt[1:nk];

\section{procedure genkracht;}

beg1n Ieal $a p, a a, a b ; a a:=f 1[k]-f 1[1] ; a b:=\{1[j]-f 1[1]$;

If abs (aa) $>1$ then aa: $=9 a-6.2832 \times 1$ sin(aa);

1f $a b s(a b)>1$ then $a b:=a b-6.2832 \times \operatorname{sign}(a b)$;

$a p:=a b s(a a x(p s i[j]-p s i[1])-a b x(p s i[k]-p s i[1])) / 6$;

$f x:=f x-a p x(p[1] \times \cos (f 1[1])+p[j] \times \cos (f 1[j])+p[k] \times \cos (f 1[k]))$;

$f y:=f y \rightarrow p x(p[1] \times \sin (f i[1])+p[j] \times \sin (f i[j])+p[k] \times \sin (f 1[k])) ;$

end Berikrachtcylinder;

\section{procedure printdruk;}

begin integer $t$; resl $x$;

nier;

for $f:=2 x n f 1$ step - 2unti1 1,1step 2until 2xnf1-1dg

beg1n nicr; for $1:=1$ step 1 until npso

begin $x:=p[1+1+n p s x(j-1)]$; if $x=$ pcavthen printtext (MMMUW P) else flot $(3,2, x)$ end ;

베옹 ;

nler; nler;

end printdruk;

\section{procedure 1terate;}

begin real fout,mxp; array c[2:nps+1];

Integer tel; tel: $: 0$;

for $1:=1+$ npsgtep -1 until $20 \mathrm{co}[1]:=0$;

l2: fout: $=0$;

tel: $=$ tel +1 ;

for $k:=2$ gtep exnpsuntil $n k, n k-n p s+1$ step $-2 x n p s u n t 1]$ npsgo

for $1:=k+n$ ng-1 step - iuntil kdo

begin real $x, y$;

$\mathrm{n}:=1-(1-2)$ inps $\times$ nps;

1 randpt $[i]$ then begin $c[n]:=0$; goto rpend ;

$x:=v[1]+c[n] ;$

for $j:=1-m$ step 1 untif $1-1$ do if $j \times$ then

$x:=x-[j,[-j] \times p[j]$;

for $j:=1+1$ step 1 untid $1+$ mof 11 jsnkthen

$x:=x-a[1, j-1] \times p[j] ; \quad y:=p[1] ;$

$p[1]:=1.7 \times x / a[i, 0]-.7 \times p[i] ;$

If $p[1]<p c \varepsilon v$ then begin real $x ; x:=v[1]+c[n] ; p[1]:=$ pcav;

for $j:=1-m g t e p$ lunt 1$]$ ddo if $j \times 0$ then $x:=x-a[j, 1-j] \times p[j]$;

for $j:=i+1$ step 1 unt 1$]$ i+mdo if $j \leq n k$ then $x:=x-a[1, j-1] \times p[j]$;

$\operatorname{mxp}:=\mathrm{frac}[1]$; 


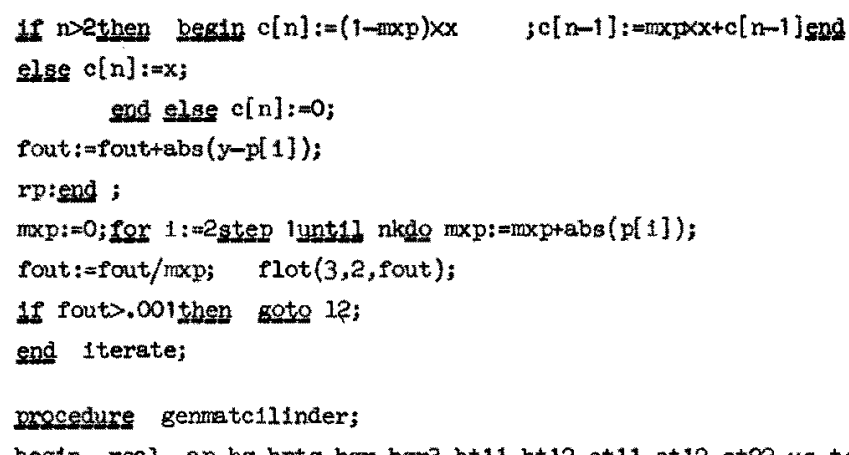

beg1n regl ep, hg, hptg, hgr, hgr 3, bt11, bt12, ct11, ct12, ct22, us, test;

integer $n, n, 11, j 1, k 1,12, j 2, k 2, s w ;$

arrey $r 1, \mathrm{re}[1: 3]$;

test: $=p s:[t]+y-8$;

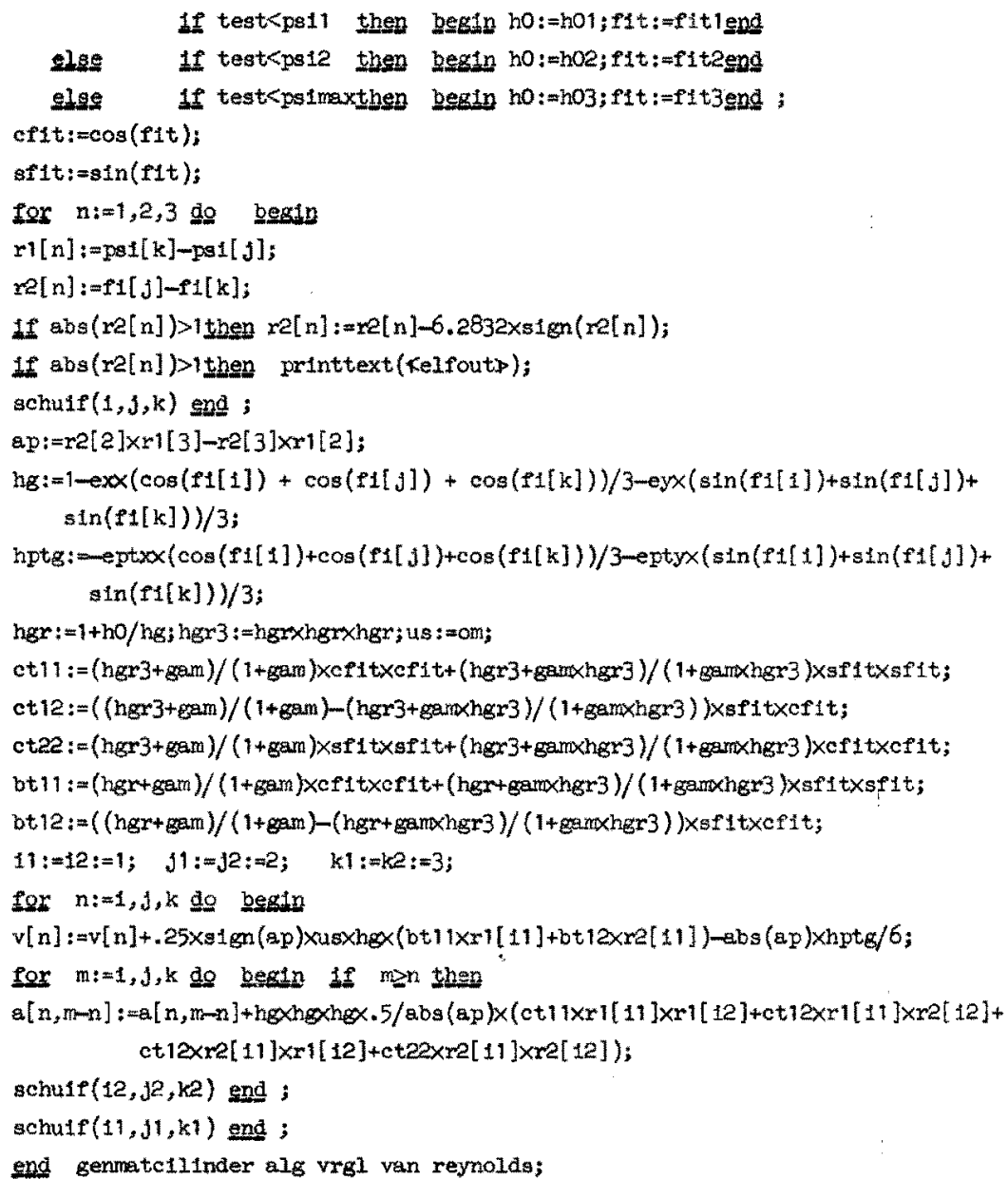


Drocedure arfrac;

begin segl hg, her, hgr3, bt11, bt12, cf1t, sf1t, test;

for 1:=1gtep lunt11 nikdo

begin test $=\operatorname{ps} 1[1]-m ;$

\begin{tabular}{|c|c|c|}
\hline & If test<psil then & beasin ho:=hol;f1t:=fitlend \\
\hline elge & If test<psia & bexin ho:=ho2; f1t:=f1t2end \\
\hline elge & if teat<psimaxthen & bestn ho:mo3; f1t:=f1t3end ; \\
\hline
\end{tabular}

cfit:=cos (fit); sfit:=sin(fit);hg:=1-exxcos(f1[1])-eyxin(fil(1]);

hgx: $=1+h 0 /$ hg; her3 : =hgrXhgrxhsr;

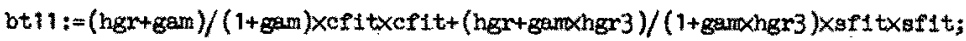

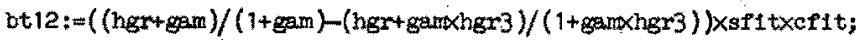

11 $1-(1-2)$ inpsxnps>2then

frac[1]:=bt12/bt11Xaf1/(ps:[1]-psi[1-1]) else frac[1]:=0;

end ;

for j:=2xnfigtep -2unti1 1, 1steg 2until axnf1-1do

begln aler; for $1:=1$ step luntil npado fixt $(0,3, f \mathrm{rac}[i+1+n p s \times(j-1)])$ end

end arfrac;

swo: $=0$;

11t:

for 1:=1 stet 1 unt1] nk do begtn for $j:=0$ step 1 unt1l m do $a[1, j]:=0 ; v[1]:=0 ; \operatorname{ranapt}[1]:=f$ glse end ;

a $[1,0]:=1 ;$

Lor $n:=2$ step 2 until exnf1-2 do el $(n, 1$, genratc1inge:);

for $n:=3$ step 2 until $2 \times n f 1$ go el( $n,-3$, genmateilinder);

el $(1,0$, genmate1linder $)$;

$\mathrm{n}:=2 \times \mathrm{nP1}$;

el $(n,-2$, sermatclilinder $)$;

For 1:=nps+1gtep npsuntil nkdo begin $p[1]:=0 ;$ ranapt[i]:=true ;

for $j:=1-$ asten lunt 1 do is j>othen begtn

$v[j]:=v[j]-p[1] \times a[j, 1-j] ; a[j, 1-j]:=0$ end ;

for $j:=1+1$ gtep 1until $1+$ mdo if $j$ snkthen begin

$v[\jmath]:=v[\jmath]-p[1] \times a[1, j-1] ; a[1, j-1]:=0$ end ;

a: 1,0$]:=1 ; v[1]:=\mathrm{p}[1]$; end ;

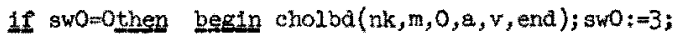

for $1:=1$ gtep lunt1] nk do if $v[1] \geq$ pcavthen $p[1]:=v[1]$ slse begin

o[ 1$]:=$ pcav; swo:=1 end end elses

1f $s w 0=1$ then begin arfrac; 1terate; $s w 0:=2$ end else

If awO>ithen begin

for $1:=1$ step luntil nkdo if $p[1]$ wpavthen begin

for $J:=1-m s t e p$ lunt1 1 do if $j \times$ othen

begin $\quad v[j]:=v[j]-p[1] \times a[j, 1-\jmath] ; a[j, 1-j]:=0$ end ;

for $J:=1+1$ step 1 unt 11 1+mdo if $J \leq n k t h e n$

begin $v[1] ;=v[\jmath]-p[1] \times a[1, j-1] ; \varepsilon[1, j-1]:=0$ end ;

$a[1,0]:=1 ; v[1]:=p[1] ;$ end ; 
cholbd(nk, m, $0, a, v$, end );

for $1:=1$ gtep luntil nkdo $\mathrm{d}[1]:=\mathrm{v}[1] ;$ nler;

printtext(kex ey eptx epty $\$$ ); $\$$ : $:=\$ \$ O+1$;

nicr;fixt $(1,2$, ex $) ; f 1 x t(1,2$, ey $) ; f 1 x t(1,2$, eptx $) ; f 1 x t(1,2$, epty $)$;

end:

printdruk;

$\mathrm{Px}_{\mathrm{x}}:=\mathrm{Py}:=0$;

for $n:=2$ ster 2 unty: $2 \times n f 1-2$ do el( $n, 1$,genkracht);

for $n:=3$ step 2 unt1] 2xnf: do el $(n,-3$, genkracht);

el $(1,0$, genkracht $)$;

$n:=2 \times n+1 ;$

el $(n,-2$, genkracht);

nicr;

printtext (K fx fy $f$ att.anglep);

nicr; $f$ lot $(5,2, f x) ; \operatorname{flot}(5,2, f y) ; f 10 t(3,2$, sqrt $(f x \times f x+f y x f y)) ;$

P1xt $(1,3, \arctan (f y / f x)) ;$ nler; new pege;

if swO=1 then soto lit;

1f swo=2 then gotg l1t;

If $\mathrm{sw} 0 \mathrm{~m}$ then begin ex:=ex+.01;

gote lit end:

1 sw0 $=4$ then begln ex:=ex-.01; ey:=ey+.01; coto itt end;

If $s w 0=5$ then begin ey: $=\mathrm{ey}-.01$; eptx: $=\mathrm{ptx}+1$; goto $11 \mathrm{t}$ end;

if $s w 0=6$ then begin eptx:=eptx-1; epty:=epty+1; Boto ilt end;

end genkrachtblok;

end:

end nloop

end programma 


\section{List of symbols}

\section{General remark}

Quantities denoted by an upper case symbol are as a rule nondimensionless; quantities denoted by a lower case symbol are in general dimensionless.

N.B. The following definitions do not apply to the floating-bush bearing. The quantities used in that case have to be defined in a slightly different way (cf. text).

\section{Mathematical symbols}

$\begin{array}{ll}\epsilon & \text { belongs to } \\ \Rightarrow & \text { does not belong to } \\ \Leftrightarrow & \text { implies that } \\ \rightarrow & \text { is equivalent to } \\ \text { symbol or symbol } & \text { approaches } \\ \text { [symbol] } & \text { vector } \\ \text { [symbol] } & \text { matrix } \\ \{\text { symbol }\} & \text { transposed matrix, [symbol] } \\ m \times n \text { matrix } & \text { column matrix } \\ \nabla & \text { matrix with } m \text { rows and } n \text { columns } \\ \nabla . & \text { gradient operator } \\ \delta & \text { divergence operator }\end{array}$

(symbol) $=\frac{\mathrm{d}}{\mathrm{d} \tau}($ symbol) dimensionless time derivative

Reference parameters (and how they are normally chosen in this work)

$L_{0}(R)$

$H_{0}(\Delta R)$

$\Omega_{0}(\Omega)$

$P_{0}\left(\frac{12 \eta \Omega R^{2}}{(\Delta R)^{2}}\right)$ reference bearing dimension

reference film thickness

reference angular velocity

reference pressure 
$F_{0}\left(\frac{12 \eta \Omega R^{4}}{(\Delta R)^{2}}\right) \quad$ reference force

Note: In chapter $2, F_{0}=W$, the radial bearing load, was chosen.

$Q_{0}(\Omega \Delta R R)$ reference flow

\section{Coordinates}

$\mathbf{E}, \mathbf{e}=\frac{\mathbf{E}}{\Delta R}$ equilibrium position of journal with respect to the bearing bush

$\mathbf{E}_{s}, \mathbf{e}_{s}=\frac{\mathbf{E}_{s}}{\Delta R}$ equilibrium position of support with respect to the frame

$\mathbf{e}_{\mathrm{tot}}=\mathbf{e}+\mathbf{e}_{s}$ equilibrium position of journal with respect to the frame

$e=|\mathbf{e}|$ length of $\mathbf{e}$

$e_{x}, e_{y}, e_{s x}, e_{s y}$ $x$-components and $y$-components of $\mathbf{e}$ and $\mathbf{e}_{s}$

$\mathbf{X}_{b}, \mathbf{x}_{b}=\frac{\mathbf{X}_{b}}{\Delta R}$ dynamic deflection of journal with respect to the bush

$\mathbf{X}_{s}, \mathbf{x}_{s}=\frac{\mathbf{X}_{s}}{\Delta R}$

dynamic deflection of support with respect to the frame

$\mathbf{X}, \mathrm{x}=\frac{\mathrm{X}}{\Delta R}=\mathbf{x}_{b}+\mathbf{x}_{s} \quad$ dynamic deflection of journal with respect to the frame

$X_{b}, Y_{b}, x_{b}, y_{b}$ components of $X_{b}$ and $x_{b}$

$X_{s}, Y_{s}, x_{s}, y_{s}$ components of $\mathbf{X}_{s}$ and $\mathbf{X}_{s}$

$X, Y, x, y$ components of $\mathbf{X}$ and $\mathbf{x}$

$X_{f}, x_{f}=\frac{X_{f}}{L_{0}}$

$Y_{f}, y_{f}=\frac{Y_{f}}{L_{0}}$

$Z_{f}$

$\Phi, \varphi=\frac{\Phi}{L_{0}}$

$\Psi, \psi=\frac{\Psi}{L_{0}}$

local coordinates of the lubricant film

(global) coordinates of the film region 
Quantities related to the dynamics of the system

$\begin{array}{ll}M_{R} & \begin{array}{l}\text { mass of the rotor } \\ \text { apparent mass of the rotor } \\ M_{R}{ }^{*}\end{array} \\ J & \begin{array}{l}\text { moment of inertia of the rotor in relation to the axis } \\ \text { of rotation } \\ \text { moment of inertia of the rotor in relation to an axis } \\ \text { through the centre of gravity and perpendicular to the } \\ \text { axis of rotation }\end{array} \\ J^{*} & \begin{array}{l}\text { apparent moment of inertia } \\ \text { mass of the support } \\ M_{S}\end{array} \\ \Omega_{w}, \omega_{w}=\frac{\Omega_{w}}{\Omega_{0}} & \begin{array}{l}\text { angular speed of the rotor } \\ t, \tau=\Omega_{0} t\end{array} \\ L & \begin{array}{l}\text { angular whirl velocity } \\ \text { time, dimensionless time } \\ \text { distance of bearings } \\ \text { force acting on journal when rotor is in equilibrium }\end{array} \\ \text { position }\end{array}$

$\Delta \mathbf{F}, \Delta \mathbf{f}=\frac{\Delta \mathbf{F}}{F_{0}} \quad$ dynamic force acting on journal, rigid support

$\Delta \mathrm{F}_{b}, \Delta \mathrm{f}_{b}=\frac{\Delta \mathrm{F}_{b}}{F_{0}} \quad$ dynamic force acting on journal, flexible support

$\Delta \mathbf{F}_{s}, \Delta \mathbf{f}_{s}=\frac{\Delta \mathrm{F}_{s}}{F_{0}} \quad$ dynamic force acting on the bush

$\Delta f_{b x}, \Delta f_{b y}, \Delta f_{s x}$, etc. components of the vectors $\Delta \mathbf{f}_{b}, \Delta \mathbf{f}_{s}$, etc.

$\alpha_{s} \quad$ phase angle of the support response

$A_{x x}=-\frac{\partial \Delta F_{x}}{\partial X}, A_{x y}=-\frac{\partial \Delta F_{x}}{\partial Y}$, etc. $\quad$ stiffness coefficients of bearing,

$a_{x x}=\frac{\Delta R}{F_{0}} A_{x x}, \quad a_{x y}=\frac{\Delta R}{F_{0}} A_{x y}, \quad$ etc. $\quad$ rigid support

$A_{b x x}=-\frac{\partial \Delta F_{b x}}{\partial X_{b}}, A_{b x y}=-\frac{\partial \Delta F_{b y}}{\partial Y}$, etc. / stiffness coefficients of bearing,

$a_{b x x}=\frac{\Delta R}{F_{0}} A_{b x x}, \quad a_{b x y}=\frac{\Delta R}{F_{0}} A_{b x y}, \quad$ etc. $\{$ flexible support 


$$
\begin{aligned}
& B_{x x}=-\frac{\partial \Delta F_{x}}{\partial(\mathrm{d} X / \mathrm{d} t)}, B_{x y}=-\frac{\partial \Delta F_{x}}{\partial(\mathrm{d} Y / \mathrm{d} t)}, \text { etc. }_{\text {damping coefficients of bearing, }} \\
& \left.b_{x x}=\frac{\Omega \Delta R}{F_{0}} B_{x x}, b_{x y}=\frac{\Omega \Delta R}{F_{0}} B_{x y}, \text { etc. }\right\} \text { rigid support } \\
& \left.B_{b x x}=-\frac{\partial \Delta F_{b x}}{\partial\left(\mathrm{d} X_{b} / \mathrm{d} t\right)}, B_{b x y}=-\frac{\partial \Delta F_{b y}}{\partial\left(\mathrm{d} Y_{b} / \mathrm{d} t\right)}\right) \text { damping coefficients of bearing, } \\
& \left.b_{b x x}=\frac{\Omega \Delta R}{F_{0}} B_{b x x}, b_{b x y}=\frac{\Omega \Delta R}{F_{0}} B_{b x y} \text {, etc. }\right\} \text { flexible support } \\
& A_{s x x}, A_{s x y} \\
& a_{s x x}=\frac{\Delta R}{F_{0}} A_{s x x}, a_{s, x y}=\frac{\Delta R}{F_{0}} A_{s x y}
\end{aligned}
$$

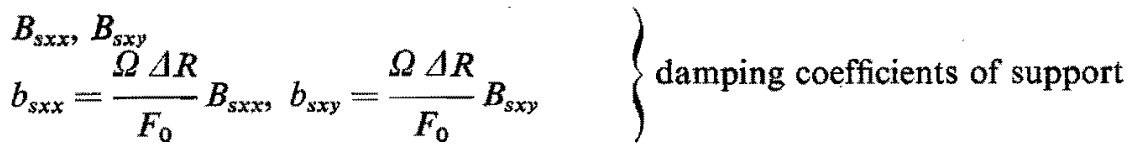

$$
\begin{aligned}
& k=\frac{a_{\text {sxx }}}{\bar{W}} \quad \text { stiffness coefficient of support } \\
& \varrho=b_{s x x} \quad \text { damping coefficient of support } \\
& \zeta=\frac{a_{s y y}}{a_{s x x}} \quad \text { asymmetry in support stiffness } \\
& \xi=\frac{b_{s y y}}{b_{s x x}} \quad \text { asymmetry in support damping } \\
& W=\left\{\begin{array}{ll}
\frac{2 F_{0}}{M_{R} \Delta R \Omega^{2}} & \text { (translational mode) } \\
\frac{F_{0} L^{2}}{2 J \Delta R \Omega^{2}} & \text { (conical mode) }
\end{array}\right\} \text { stability parameter } \\
& \lambda=\left\{\begin{array}{ll}
0 & \text { (translational mode) } \\
\frac{I}{J} & \text { (conical mode) }
\end{array}\right\} \text { gyroscopic parameter } \\
& m_{s}=\left\{\begin{array}{ll}
\frac{2 M_{S}}{M_{R}} & \text { (translational mode) } \\
\frac{M_{S} L^{2}}{2 J} & \text { (conical mode) }
\end{array}\right\} \text { mass of support }
\end{aligned}
$$


Quantities related to the bearing

$D$

$R$

$B$

$\Delta R$

$\eta$

$L_{g}, l_{g}=\frac{L_{g}}{L_{0}}$

$L_{g}$

$H_{s}, h_{s}=\frac{H_{s}}{H_{0}}$

$\alpha$

$\gamma$

$k_{g}$

$\varphi_{a}$

$f_{x}=\frac{F_{x}}{F_{0}}, f_{y}=\frac{F_{y}}{F_{0}}, f_{z}=\frac{F_{z}}{F_{0}}$

$f=\left(f_{x}^{2}+f_{y}^{2}\right)^{1 / 2}=\frac{W}{F_{0}}=\frac{F}{F_{0}}$ diameter

radius

length

radial clearance

viscosity of lubricant (nondimensionless)

period of grooves (in chapter 5)

length of the grooved part of a cylindrical bearing (in chapters 6 and 7)

depth of grooves

groove angle

ridge-to-groove ratio

number of grooves

attitude angle

dimensionless bearing reactions in in $X-, Y$ - and $Z$-directions

dimensionless (radial) load capacity

Quantities related to the lubricant film and the FEM

$n$

$k$

$G$

$R$

$G_{k}$

$R_{k}$

$R_{a}$

$R_{b}$

$R_{c}$

$R_{d}$

$q_{n}$ number of nodal points

number of elements

film region

boundary of $G$

sub-region, element

boundary of $G_{k}$

section of $R$ with prescribed pressure

section of $R$ with prescribed outflow

section of $R$ with "periodicity"

section of $R$ with outflow proportional to pressure

dimensionless outflow per unit length $\left(R_{b}\right)$ 
$q_{\text {tot } n}$

$\lambda_{b}$

$P, p=\frac{P}{P_{0}}$

$P_{\mathrm{sm}}, p_{\mathrm{sm}}=\frac{P_{\mathrm{sm}}}{P_{0}}$

$P_{\text {cav },} p_{\text {cav }}=\frac{P_{\text {cav }}}{P_{0}}$

$\{P\},\{p\}=\frac{1}{P_{0}}\{P\}$

$P_{k}, p_{k}$

$\mathbf{Q}, \mathbf{q}=\frac{\mathbf{Q}}{Q_{0}}$

$Q_{x}, q_{x}$, etc.

$\mathbf{Q}_{\mathrm{sm}}, \mathbf{q}_{\mathrm{sm}}=\frac{\mathbf{Q}_{\mathrm{sm}}}{Q_{0}}$

$Q_{\mathrm{sm} x}, q_{\mathrm{sm} x}$, etc.

$U_{x}^{*}, U_{y}^{*}$

$\mathbf{U}, \mathbf{u}=\frac{\mathbf{U}}{\Omega_{0} L_{0}}$

$U_{x}, u_{x}$

$\{u\}=\left\{\begin{array}{l}u_{x_{f}} \\ u_{y_{f}}\end{array}\right\}$

$\left\{f_{k}\right\}$

$f_{k i}$

$\left[r_{k}\right]$

$[a]$

$\{v\}$

$\{q s\}$

$\{q n\}$

[al] dimensionless total outflow $\left(R_{b}\right)$

reciprocal value of flow resistance at $R_{d}$

local pressure

smoothed pressure

cavitation pressure

column matrix of nodal pressures

elements of $\{P\}$ and $\{p\}$

local flow

$x$-components of $\mathbf{Q}$ and $\mathbf{q}$, etc.

smoothed flow

$x$-components of $\mathbf{Q}_{\mathrm{sm}}$ and $\mathbf{q}_{\mathrm{sm}}$, etc.

$x$ - and $y$-components of local velocity in the film

velocity of moving surface

$x$-components of $\mathbf{U}$ and $\mathbf{u}$

column matrix of surface velocities in system $X_{f}, Y_{f}, Z_{f}$

matrix of interpolation functions of element $G_{k}$ element of $\left\{f_{k}\right\}$ related to nodal point $i$

$2 \times n$ matrix of the interpolation functions of the pressure gradients in $G_{k}$

$n \times n$ fluidity matrix

$n \times 1$ surface-velocity matrix

$n \times 1$ squeeze matrix

$n \times 1$ boundary matrix

$n \times n$ matrix, denoting normal flow proportional to boundary pressure 


\section{Summary}

An attractive feature of sliding bearings is that in normal conditions of operation they are free from mechanical wear, the bearing surfaces being completely separated from each other by a film of lubricant. This feature is of importance in, above all, high-speed rotor-and-bearing systems required to comply with the specification of a relatively long service life. An objection to these bearings is, however, that mastery of the dynamics of the rotor, in particular the prevention of rotor instability, requires a great deal of attention. The purpose of the work here reported was to widen our understanding of the stability of such systems.

The first part treats the stability of a number of "symmetrical" rotor-and-bearing systems; the symmetry makes it possible to resolve the motion of the system into a translational and a conical mode. The gyroscopic effects occurring in the conical mode of motion appear, in most cases, to exert a stabilising influence on this motion. Forces of unbalance, too, are found to have a stabilising effect in the single case so far examined. With the particular kind of bearings having a rotationally symmetrical response it turns out that, if moreover both the rotor and the bearing supports are rigid, an easily handled criterion of the stability can be established in respect of either mode of motion. This criterion makes it possible to say something, also, about the stability if the excursions from the equilibrium position become so great that the bearing response can no longer be assumed to be linear.

With rigid rotors having flexibly supported bearings it is found that the parameters of the support greatly influence the stability:

- With a support consisting of springs and dampers it is possible in certain cases to indicate limits of the support damping, within which the rotor is inherently stable.

- With a support consisting of an "adjoint" bearing, e.g. a floating-bush bearing, the theory provides an indication of the optimum choice of the parameters in respect of stability, so that new designs of this kind of bearing suggest themselves.

For an examination of the dynamics of a rotor it is desirable to know the bearing response. Therefore in the second part of the work (chapters 5 and 6 ) the versatile finite-element method has been developed for the calculation of such response characteristics as load capacity, attitude angle, and response coefficients of sliding bearings lubricated with a Newtonian liquid. In the finiteelement method a generalised Reynolds equation can be applied to the flow between a grooved and a grooveless surface. Further the phenomenon of cavitation, of great importance in the case of liquid-lubricated bearings, can now be taken into account in a way in which, in contrast to the conventional methods, the flow balance is maintained. 
The last part of this work deals with the calculation of several types of cylindrical and spherical sliding bearings: the data required for a stability analysis, such as response coefficients, are represented graphically. An idea of the effect of the calculated bearings on the stability of a rotor-and-bearing system has been gained from the determination of the stability of a rigid rotor with rigidly supported bearings.

The present work points to possibilities of achieving more stable rotor-andbearing systems, for example:

- by the use of special stabilising bearing supports,

- by developing, with the help of the finite-element method, new bearings with good stability characteristics. 


\section{Levensbericht}

De schrijver van dit proefschrift werd op 25 februari 1936 geboren te Vlissingen. Hij bezocht daar de H.B.S. (diploma 1953) en de H.T.S.-wtb (diploma 1956).

In 1956 trad hij in dienst bij het Natuurkundig Laboratorium der N.V. Philips' Gloeilampenfabrieken.

Tot 1965 was hij, met een onderbreking voor de Militaire Dienstplicht (van 1957 tot 1959), werkzaam in de researchgroep die de z.g. Stirlingcyclus onderzocht.

In 1959 begon hij als werkstudent de studie voor werktuigbouwkundig ingenieur aan de Technische Hogeschool te Eindhoven en behaalde in 1963, nadat hem een jaar studieverlof was verleend, het diploma met lof.

Sinds 1965 heeft hij onderzoek verricht op het gebied der Technische Mechanica; één van de onderzoeken is in dit proefschrift beschreven. 
STELLINGEN

bij het proefschrift van

J. P. Reinhoudt 
Voor lagerexcentriciteiten kleiner dan ca. 0.3 (en $B / D=1)$ heeft een zelfwerkend cilindrisch lager, dat voorzien is van een ,optimaal" groevenpatroon, een hogere draagkracht en betere stabiliteits-eigenschappen dan een glad lager; bij hogere excentriciteiten is het gladde lager echter in het voordeel.

Dit proefschrift pag. 120 .

II

Wanneer het middelpunt van de tap van een cilindrisch lager om het middelpunt van de bus cirkelt met de karakteristieke hoeksnelheid, treedt normaliter geen cavitatie op.

De karakteristieke hoeksnelheid is gedefinieerd als de hoeksnelheid van het tapmiddelpunt, waarbij de tangentiale component van de lagerresponsie gelijk is aan nul.

Dit proefschrift pag. 23.

III

De bewering van Chow en Vohr dat het voor een gegroefd lager, gesmeerd met een onsamendrukbaar smeermiddel, niet uitmaakt of de groeven in het roterende deel of in het stilstaande deel zijn aangebracht, is onjuist.

C. Y. Chow, J. H. Vohr, Helical-grooved journal bearing operated in the turbulent regime.

Trans. ASME, J. Lub. Techn. 92, 346-358, 1970.

\section{IV}

De z.g. impuls-whirl angle methode kan worden uitgebreid tot gegroefde lagers.

College Prof. Ir. H. B lok. J. F. Booker, Dynamically Loaded Journal Bearings: Mobility Method of Solution, Trans. ASME, J. B. Eng. 87, sept. 1965.

$\mathrm{V}$

Indien flexibele folie met een zeker spanningsverschil over een starre roterende rol wordt getransporteerd, zal slip tussen rol en folie optreden in het gebied waar de folie de rol verlaat.

\section{VI}

Sommige "versterkte" materialen hebben een ingebouwd destructie-mechanisme. 


\section{VII}

De mogelijkheid om de afvalwarmte van elektrische centrales voor verwarmingsdoeleinden te gebruiken verdient opnieuw alle aandacht; een dergelijk gebruik immers kan het bezwaar van thermische energie conversie - dat grote hoeveelheden warmte verloren gaan - aanzienlijk verkleinen.

\section{VIII}

Het verdient aanbeveling de responsie van de maatschappij op de invoering van een nieuwe maatregel, bijv. een sociale wet, met behulp van computermodellen te onderzoeken en, in analogie met de term ,world dynamics", de term "law dynamics" in te voeren.

\section{IX}

Evenals dit het geval is bij industriële productie, kan bij wetenschappelijke opleidingen de efficiency worden verhoogd door typebeperking.

\section{$\mathrm{X}$}

De vouwfiets dient zodanig te worden geconstrueerd dat hij als handbagage in een openbaar vervoermiddel meegenomen kan worden. 